\title{
Identification of chicken repeat 1 (CR1) elements in forensically important carrion fly species and characterization of one such element within Sarcophaga bullata
}

Michelle Lee Thompson

West Virginia University

Follow this and additional works at: https://researchrepository.wvu.edu/etd

\section{Recommended Citation}

Thompson, Michelle Lee, "Identification of chicken repeat 1 (CR1) elements in forensically important carrion fly species and characterization of one such element within Sarcophaga bullata" (2009). Graduate Theses, Dissertations, and Problem Reports. 4543.

https://researchrepository.wvu.edu/etd/4543

This Thesis is protected by copyright and/or related rights. It has been brought to you by the The Research Repository @ WVU with permission from the rights-holder(s). You are free to use this Thesis in any way that is permitted by the copyright and related rights legislation that applies to your use. For other uses you must obtain permission from the rights-holder(s) directly, unless additional rights are indicated by a Creative Commons license in the record and/ or on the work itself. This Thesis has been accepted for inclusion in WVU Graduate Theses, Dissertations, and Problem Reports collection by an authorized administrator of The Research Repository @ WVU. For more information, please contact researchrepository@mail.wvu.edu. 
Identification of Chicken Repeat 1 (CR1) elements in forensically important carrion fly species and characterization of one such element within Sarcophaga bullata.

Michelle Lee Thompson

Thesis submitted to the Eberly College of Arts and Sciences at West Virginia University in partial fulfillment of the requirements

for the degree of

Master of Science

in

Genetics and Genome Biology/

Forensic Biology

David A. Ray, Ph.D, Chair

Jeffrey D. Wells, Ph.D

Stephen P. DiFazio, Ph.D

Department of Biology

Morgantown, West Virginia 2009

Keywords: Mobile Elements, CR1, Carrion flies, Forensic Identification

Copyright 2009, Michelle L. Thompson 


\begin{abstract}
Identification of Chicken Repeat 1 (CR1) elements in forensically important carrion fly species and characterization of one such element within Sarcophaga bullata.
\end{abstract}

Michelle Lee Thompson

Retrotransposons including CR1 (Chicken Repeat 1) elements mobilize in a genome in a way that makes them useful for phylogenetic analysis and species identification. This study was designed to identify lineages of CR1 elements in the genomes of forensically important carrion fly species and to further characterize the one element, Sarcophaga bullata CR1B, though genome walk techniques.

Using conserved oligonucleotide primers, CR1 fragments from several carrion fly taxa were amplified, cloned and sequenced, and the recovered sequences were analyzed to identify different lineages of elements. A variety of retrotransposon families were recovered that exhibit similarity to known retrotransposon families from other dipterans (e.g. mosquitoes, Drosophila). We also provide evidence that several of these lineages may have given rise to taxon-specific subfamilies that have been recently active in carrion fly genomes.

The final reconstructed sequence from the $S$. bullata CR1B genome walk is presumed to be the complete Open Reading Frame 2 (ORF2) portion of the element containing both the reverse transcriptase (RT) and endonuclease (EN) domains. These domains were used to identify conserved amino acid regions in the recovered consensus via comparison to known non-LTR retrotransposons. A phylogenetic analysis of the RT domain revealed the recovered ORF in S. bullata compares favorably with previously documented CR1-like elements.

This work will serve as the basis for additional analyses targeted at developing a simple, efficient marker system for the identification of forensically important carrion flies. The fast turnaround time possible for this system will also help to make it an extremely useful tool. Though much work will be needed to completely develop the method, the data presented here lays the groundwork for future efforts. 


\section{ACKNOWLEDGEMENTS}

First and foremost I would sincerely like to thank Dr. David Ray for all of his guidance and patience through my research here at WVU. He not only provided academic aid, he made life in his laboratory an enjoyable experience which I will forever be grateful for. I would also like to thank Dr. Jeff Wells for all of his leadership and for easing me into my first conference experience. Dr. Stephen DiFazio was also an integral part of my thesis work, and I thank him for always being willing to take time to help me when I showed up at his door. In addition I would like to thank my fellow Ray lab members as well as other friends in the department for all of their help and friendship. I do not know how I could have made it through graduate school without your comic relief and companionship. Additionally I would like to thank the Biology Department staff members and the Academic Lab managers for all of their help.

Lastly I would like to thank my parents John and Toni for all of their support and love. You have been exceptional parents while guiding me through life's endeavors. My accomplishments would not be possible without what you have taught me and I hope to continue to make you proud. 


\section{TABLE OF CONTENTS}

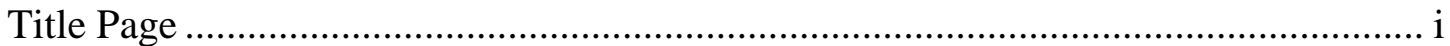

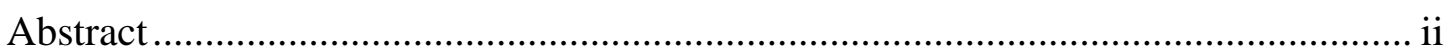

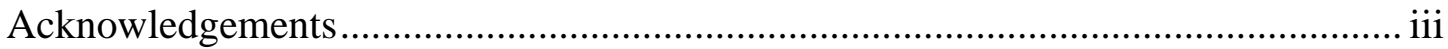

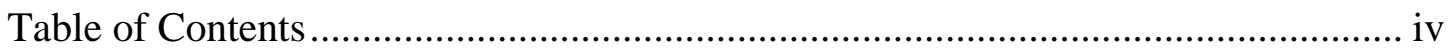

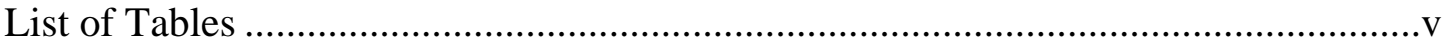

List of Figures .......................................................................................... vi

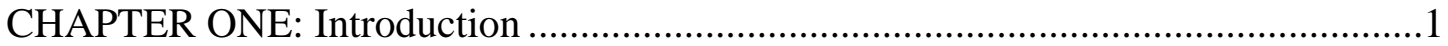

CHAPTER TWO: Identification of Chicken Repeat 1 (CR1) elements in carrion

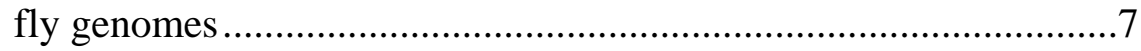

CHAPTER THREE: Characterization of CR1 element identified within Sarcophagidae ............................................................21

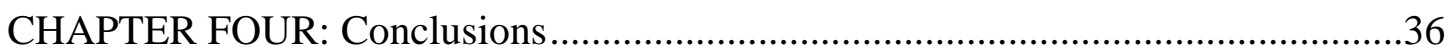

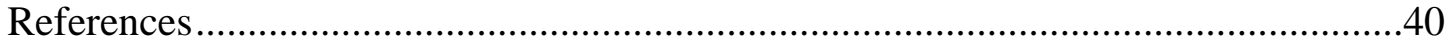

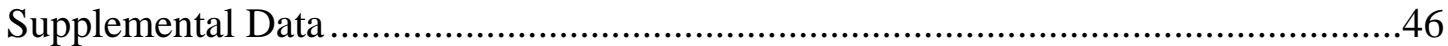




\section{LIST OF TABLES}

Table 2.1 Results of Modeltest analysis

Table 2.2 Number of sequences obtained along with number of consensus sequences obtained from them for each examined taxa....

Table 2.3 Similarity results as given by BlastX of CR1-like nucleic acid consensus sequences to known CR1 amino acid sequences .................................................... 16

Table 2.4 Divergence and standard deviation values for each cluster of elements .....19

Table 3.1 Sequence of primers used in conjunction with the GenomeWalker Universal

Kit protocol to collect data for each round of the genome walk 


\section{LIST OF FIGURES}

Figure 1.1 Schematic representation describing the general structure of transposable

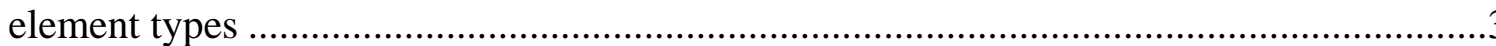

Figure 1.2 Schematic representation of agarose electrophoresis illustrating speciesspecific banding pattern differences due to a transposable element insertion.

Figure 2.1 Schematic representation of retrotransposition mechanism of CR1 elements

Figure 2.2 Gel electrophoresis results illustrating amplification of CR1- like fragments in two taxa L. sericata (Lane 2) and C. vomitoria (Lane 3). Lane 1 contains a $1 \mathrm{~kb}$ DNA ladder for reference

Figure 2.3 Phylogenetic tree inferred from translated carrion fly consensus sequences and several outgroups comprising known CR1-like elements. Carrion fly taxa are highlighted in red. The +2 indicates the reading frame for recovered sequences when compared to the original consensus sequence

Figure 3.1 A neighbor joining tree of 11 major clades of non-LTR elements inferred from the reverse transcriptase domains encoded by them (Malik, Burke et al. 1999) ......25

Figure 3.2 Original primer pairs (blue arrows) developed for both the 5' and 3' direction of the element. Numbers indicate position of the base pairs relative to the full element. Black lines below the primers indicate the resultant fragments and their relative positions inferred after sequence analysis. These sequences were used to develop new primers for additional genome walk rounds to expand our analysis of the CRI-like element........27

Figure 3.3 Alignment of S. bullata CR1B ORF2 with sequences of known non-LTR RT finger/palm region. Areas shaded in black are identical while areas shaded in grey show $90 \%$ similarity. Numbers above sequence denote the seven highly conserved areas previously described

Figure 3.4 Results of a neighbor joining phylogenetic analysis of the RT domain of CR1like non-LTR elements with the RT portion of the recovered element from S. bullata. Values on nodes are the proportion of bootstrap replicates supporting that node

Figure 3.5 Sequence alignment showing similarity of a portion from S. bullata to known EN domains. Accession numbers and species of origin are shown alongside each sequence. 
Chapter 1: Introduction 
Any genetic unit that possesses the capability of moving or copying itself throughout the genome is described by the basic term transposable element (TE). Also known as “jumping genes”, first described by Barbra McClintock in her work with maize, TE insertion events can change gene expression patterns and structural features of genomes thereby serving as a driving force of evolution (McClintock 1956). This ability to change the landscape of the genome is precisely why transposable elements have become a hot research topic (Kidwell and Lisch 1997). We now know high proportions of eukaryotic genomes consist of transposable elements. For example about $40 \%$ of the human genome is made up of TEs (Lander, Linton et al. 2001).

Transposable elements are classified into two categories based on their method of movement (Figure 1.1). Class 1 elements utilize a “copy and paste” method involving an RNA intermediate where as Class 2 elements typically mobilize via a “cut and paste” mechanism (Mizuuchi 1992; Deininger and Batzer 2002; Wessler 2006). Within Class 1, elements can be classified into Long Terminal Repeat (LTR) retrotransposons and nonLTR retrotransposons.

Non-LTR retrotransposons can be further subdivided into two families: Long INterspersed Elements (LINEs), and their non-autonomous counterparts, the Short INterspersed Elements (SINEs). Our current understanding suggests that SINEs mobilize by 'hijacking' the machinery encoded within their autonomous partners. Briefly, SINEs utilize their A and B boxes as promoter sequences to recruit RNA Polymerase III, which will then produce an RNA transcript of the element. The transcripts are then reverse transcribed and incorporated into the genome using enzymes encoded by LINEs (Bushman 2002; Kajikawa and Okada 2002; Dewannieux, Esnault et al. 2003). 


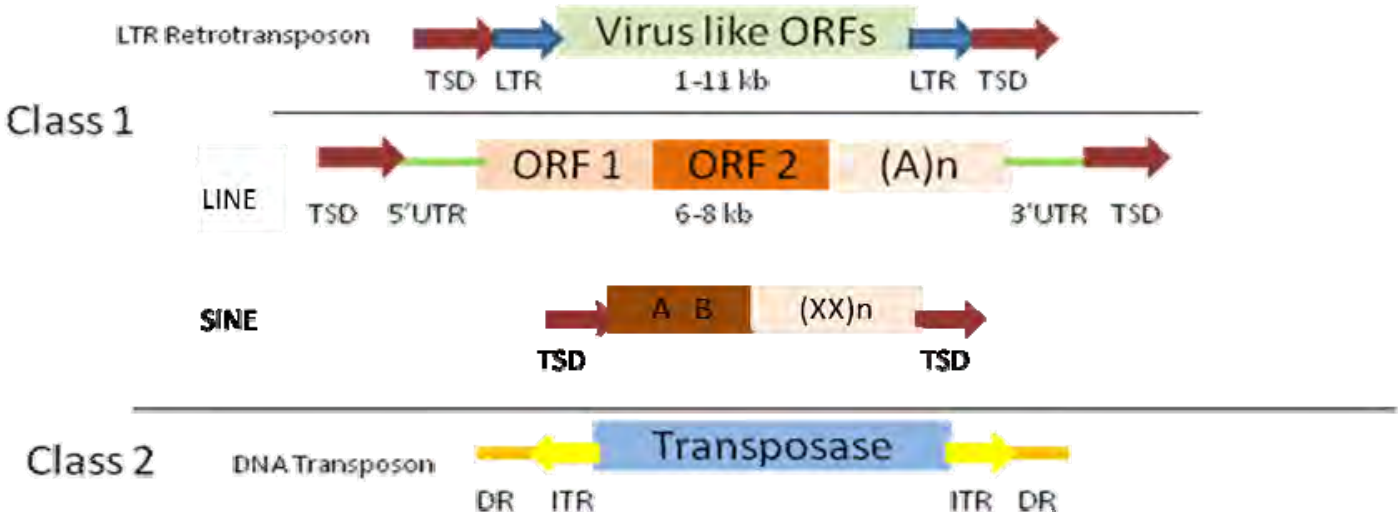

Figure 1.1 Schematic representation describing the general structure of transposable element types. Class 1 elements consist of LTR retrotransposons and non-LTR elements such as the LINE and SINE elements. These have Target site duplications (TSDs), long terminal repeats (LTRs) and or untranslated regions (UTRs) surrounding their open reading frames (ORFs) and or AB promoter sequences. Class 2 elements flanking regions have inverted terminal regions (ITRs) and direct repeats (DRs).

Class I TEs have been shown to be very reliable for species identification and for resolving species phylogeny questions (Murata, Takasaki et al. 1993; Nishihara, Satta et al. 2005; Ray, Xing et al. 2005; Kaiser, van Tuinen et al. 2007). Because of their ability to insert essentially anywhere in the genome, it is very unlikely that any two elements would insert randomly at the same site in two different genomes. This virtually eliminates the chance of identity-by-state homoplasy (Batzer and Deininger 2002; Salem, Ray et al. 2005). Simple PCR reactions with specific primers have been used to produce species-specific banding patterns and thereby establish species identity in unknown samples (Figure 1.2) (Herke, Xing et al. 2007).

The idea of using such a simple system to identify species would be especially useful in dipteran insects where it is difficult for a non-expert to identify taxa. The order Diptera is one of the largest insect orders and representatives can be found in almost 
every habitat (Byrd and Castner 2001). A major family within Diptera is Calliphoridae, members of which are collectively known as blow flies. The blow flies (1,000 species), along with the sarcophagid (2,000 species) and muscid flies (700 species), are extremely important in forensic investigations where PMI (post mortem interval) is estimated (Byrd and Castner 2001). Their importance is because they are often the first flies that come to carrion for a food source and breeding medium, and they have a life cycle that is extremely predictable (Rodriguez 1983).

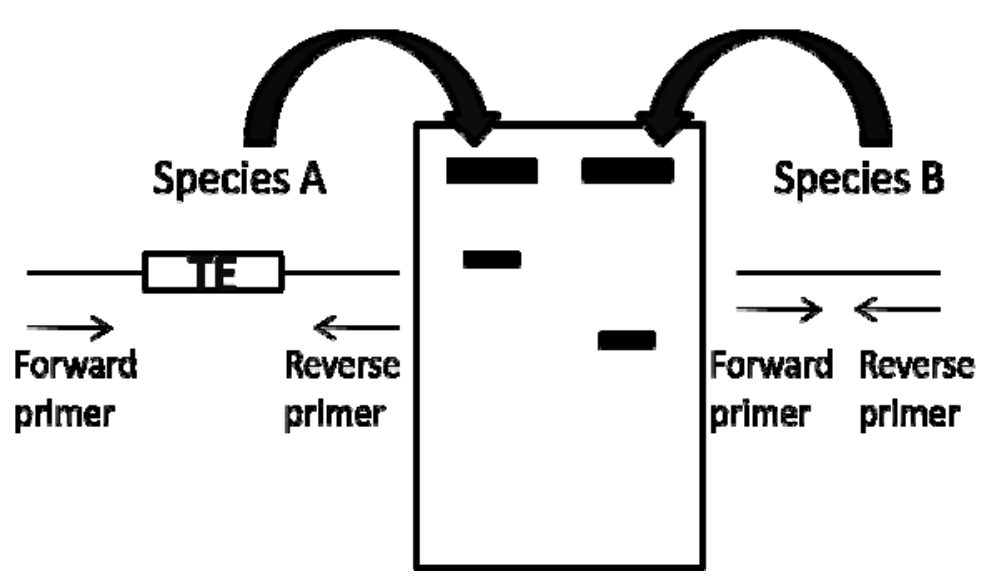

Figure 1.2: Schematic representation of agarose electrophoresis illustrating species-specific banding pattern differences due to a transposable element insertion. The larger band corresponding to species $A$ has a transposable element (TE) insertion at the specific portion of the DNA where as Species B does not and shows a shorter product that migrates farther.

An investigator is often able to establish the PMI by determining three pieces of information 1) the species retrieved from the corpse 2) the developmental stage observed, and 3) the temperatures to which the specimen was exposed.

The first piece of information, taxon identity, is extremely important to ascertaining an accurate PMI as there can be substantial differences in developmental patterns among taxa. For example, there are species such as Calliphora vicina and 
Calliphora vomitoria that display drastically different mean cumulative development times (538.4 and 572.6 hours respectively) at $20^{\circ} \mathrm{C}$ (Ames and Turner 2003). Unfortunately, distinguishing closely related taxa such as these two, especially when they are not well preserved, can be a difficult process.

When the egg or larval stage is collected from carrion it is often even more difficult to distinguish the species morphologically (Greenberg and Singh 1995). Dichotomous keys are often used to identify specimens and, though this method is generally accepted, accurate results are not guaranteed due to the substantial difficulty a non-expert may encounter during the identification process. Other problems such as specimen death can arise when the immature stage is found because it is often necessary to incubate a portion of the larva collected in order to have the opportunity of observing the adult fly. DNA-based methods, on the other hand, should better allow accurate species identification because it would be useful for both adult and larval stages of the live or dead specimens.

Identifying forensically important carrion fly species using molecular markers has been previously investigated. A study using ribosomal RNA (rRNA) has already been published; however it claims to only lay the groundwork of a marker system (Stevens and Wall 2001). Though it provides the possibly of a superior marker system, it will require high quality DNA and a turnaround of at least 48 hrs to obtain results (Stevens and Wall 2001). A method using the predicted gel banding patterns of restriction endonuclease cleaved DNA has also been developed (Sperling, Anderson et al. 1994). This method is problematic due to the possibility of mutations at the restriction site, therefore fixed or nearly fixed restriction cites are needed for each given species (Benecke and Wells 2001). 
Much work has also gone into developing mitochondrial DNA (mt-DNA) based identification methods (Sperling, Anderson et al. 1994; Wallman and Donnellan 2001; Wells, Pape et al. 2001; Wells and Sperling 2001; Otranto and Stevens 2002; Harvey, Dadour et al. 2003). As with any other method problems have been identified. It is still unknown to the investigators if the phylogenetic analysis of COI sequence method will hold up after further investigation due to paraphyly (Wells and Stevens 2008).

Primary assessment of a TE- based marker system is the focus of this thesis.

Chapter 2 is a survey of TE activity in forensically important carrion flies.

Retrotransposons are present in a variety of taxa and these elements may have been recently active. Chapter 3 describes a consensus of the complete Open Reading Frame (ORF) of a CRI-like retrotransposon found in Sarcophaga bullata. This was characterized and compared to known CR1-like retrotransposons. The element compares favorably with known CR1-like elements from a variety of taxa. It harbors many of the major functional domains typical of this family of elements. Chapter 4 details general conclusions derived from this thesis and additional applications for these findings. 
Chapter 2: Identification of Chicken Repeat 1 (CR1) elements in carrion fly genomes 


\begin{abstract}
Retrotransposons including CR1 (Chicken Repeat 1) elements mobilize in the genome in a way that makes them useful for phylogenetic analysis and species identification. This study was designed to identify lineages of CR1 elements in the genomes of forensically important carrion fly species. Using conserved oligonucleotide primers, CR1 fragments were amplified and sequenced from several carrion fly taxa and the sequences recovered were analyzed to identify different clades of elements. A variety of retrotransposon families were recovered that exhibit similarity to known retrotransposon families from other dipterans (e.g. mosquitoes, Drosophila). We also provide evidence that several of these lineages may have given rise to taxon-specific subfamilies that have been recently active in carrion fly genomes.
\end{abstract}

\title{
Introduction
}

LINEs (Long INterspersed Elements) are Class 1 transposable elements (TEs) (i.e. retrotransposons). As such, they utilize a "copy and paste” method of movement involving an RNA intermediate (Figure 2.1) (Deininger and Batzer 2002). Briefly, this process involves transcription into messenger RNA (mRNA) by RNA polymerase II, and then reverse transcription and reincorporation into the genome via a process termed Target Primed Reverse Transcription (TPRT) (Luan, Korman et al. 1993).

Endonucleases, often encoded by the elements themselves, aid in retrotransposition by cleaving the target DNA to form a 3'-OH primer. Because this process involves the introduction of a new DNA fragment without the loss of the original insertion, LINE elements tend to accumulate in genomes and are found to comprise large 
portions of the genomes they inhabit. For instance LINE-1 elements comprise about 20\% of the human genome (Lander, Linton et al. 2001).

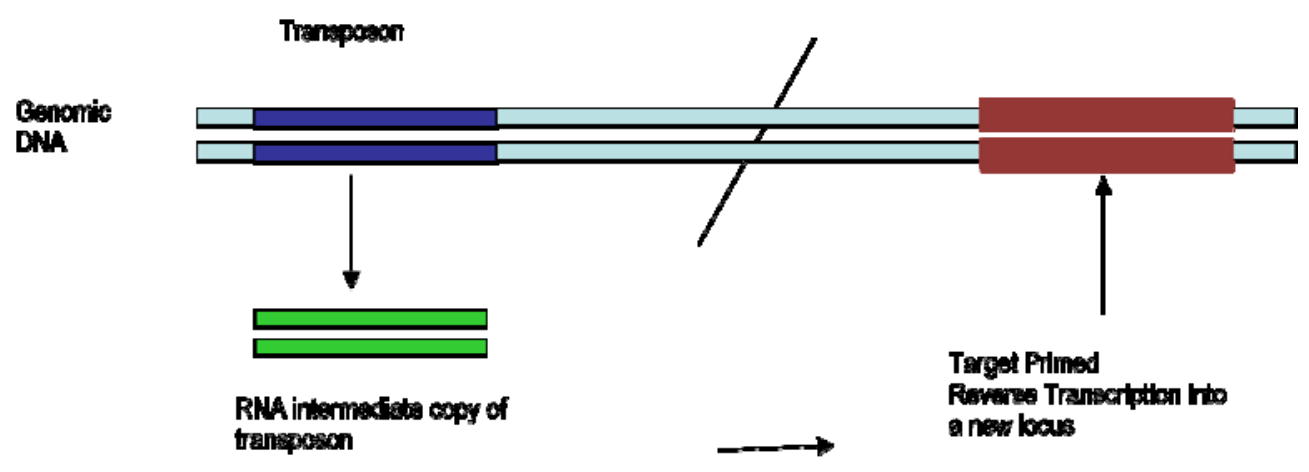

Figure 2.1 Schematic representation of retrotransposition mechanism of CR1 elements

LINE elements are thought to follow the master gene model of amplification in the genome (Vandergon and Reitman 1994). Briefly, this model suggests that retrotransposons in a given family will have only one or very few active 'master' elements at any given time. As such, any retrotransposition events will give rise to elements that are identical to the master element from which they arose. Therefore, if we find elements that are essentially identical to one another (i.e. have low divergence values compared to the consensus), they are likely the result of recent retrotransposition from a common source.

Several studies have been published assessing LINE diversity, which has resulted in their separation based on the number and characteristics of their Open Reading frames (ORF's). Further classification is made based on the reverse transcriptase (RT) portion resulting in several clusters (Malik, Burke et al. 1999). The major groups of elements identified thus far are CRE, R2, R4, LINE-1, RTE, Tad, R1, LOA, I, Jockey, and 
Chicken Repeat 1 (CR1) (Malik, Burke et al. 1999). This study will focus one particular group of LINEs, the CR1-like elements.

CR1-like elements are a widely distributed family of LINEs that have been identified in a variety of organisms including vertebrates -e.g. chicken (Haas, Grabowski et al. 1997) and turtle (Kajikawa, Ohshima et al. 1997) and invertebrates. Invertebrates with CR1-like TEs include the roundworm Caenorhabditis elegans (Jurka, Kapitonov et al. 2005), the sea urchin Strogylocentrotus purpuratus (Jurka, Kapitonov et al. 2005), and the human blood fluke Schistosoma mansoni (Drew and Brindley 1997). Arthropods known to harbor CR1-like elements include Order Scorpiones (scorpions) (Glushkov, Novikova et al. 2006), and Maculinea (butterflies) (Novikova, Sliwinska et al. 2007), as well as the dipteran family Drosophila (Biedler and Tu 2003). Until now, the LINE landscape of carrion fly genomes (Diptera: Calliphoridae and Sarcophagidae) has gone uninvestigated.

Because of this lack of information and because we are interested in the eventual utilization of LINE elements for phylogenetic analysis and species identification, the goals of this study were; 1 ) to determine whether CR1-like elements are a component of representative carrion fly genomes, 2) to categorize those elements into the known CR1like families, and 3) to determine CR1 relative divergence values and thereby estimate the potential for recent activity. The eventual development of a reliable transposable element based species identification system is one additional goal. 


\section{Materials and Methods}

DNA was extracted from six carrion fly taxa representing two families of forensically important flies - Calliphora vicina, Calliphora vomitoria, Phormia regina, Cochliomyia macellaria, and Lucilia sericata of family Calliphoridae, and Sarcophaga bullata of family Sarcophagidae. Adult specimens were collected from the wild using raw liver as bait and a standard sweep net. Species identifications were confirmed using a dichotomous key (Whitworth 2006) and specimens were preserved in 95\% ethanol overnight for DNA extraction the following day. The fly was dissected to obtain the thoracic muscle tissue and DNA extraction of that tissue was accomplished using the Qiagen Puregene Tissue Core Kit A (Gentra, Minneapolis MN).

CR1 primer pairs (CR1-S and CR1-A) had been previously developed by Novikova et al. (2007) for the identification of CR1 elements in butterflies (Lepidoptera: Maculinea). We used these to amplify a portion of an ORF from each species. PCR amplification was conducted in $25 \mu \mathrm{l}$ reactions using 50 ng of target DNA, 7 pM of each oligonucleotide primer, 200 mM dNTPs, in 50 mM KCl, 10 mM Tris-HCl (pH 8.4), 2.0 $\mathrm{mM} \mathrm{MgCl} 2$, and Taq DNA polymerase (1.25 units). This mix was then subjected to 32 cycles of $95^{\circ}$ denaturation for 30 seconds, $56^{\circ}$ anneling for 30 seconds, and a 2 min extension at $72^{\circ}$. A final $72^{\circ}$ extension was done for seven minutes. After agarose gel electrophoresis and UV visualization using ethidium bromide, the resulting amplicons (Figure 2.2) were cut from a $2 \%$ agarose gel with $0.25 \mu$ g ethidium bromide and cleaned using the Wizard SV gel and PCR clean up system (Promega, Madison WI). 


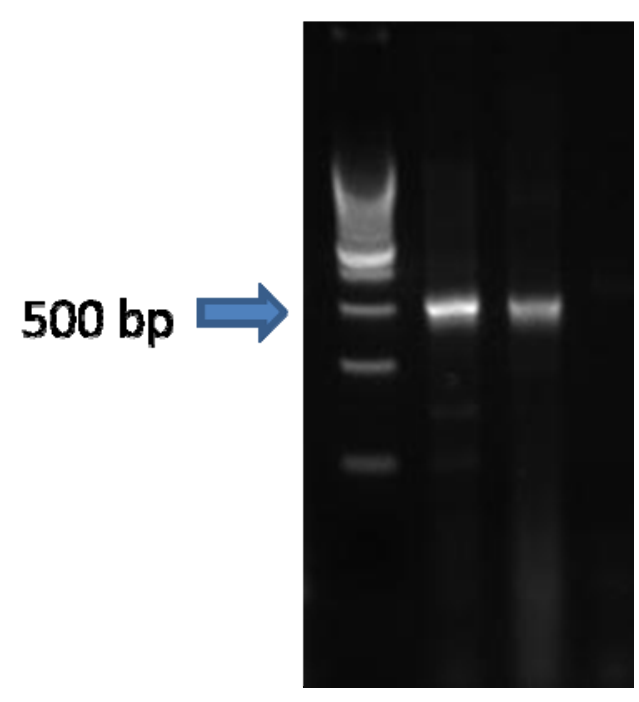

Figure 2.2 Gel electrophoresis results illustrating amplification of CR1- like fragments in two taxa $L$. sericata (Lane 2) and $C$. vomitoria (Lane 3). Lane 1 contains a $1 \mathrm{~kb}$ DNA ladder for reference.

The fragments were cloned into the TOPO TA (Invitrogen, Carlsbad CA) vector and transformed into Top10 cells using the manufacturer's protocol. Colonies were grown on $\mathrm{LB} /$ Kanamycin $(2 \mu \mathrm{l} / \mathrm{ml})$ plates overnight. Individual colonies were grown in LB/Kanamycin $(2 \mu \mathrm{l} / \mathrm{ml})$ broth overnight and plasmids were isolated using Fast Plasmid Mini kit (Eppendorf, Hamburg GER). Purified plasmids were sequenced using Big Dye termination sequencing on an ABI 3130xl Genetic Analyzer with M13 primers. Sequences used for this work have been deposited in GenBank under accession numbers FJ940924 through FJ941019.

Vector sequence was removed from the resulting data using SeqMan (DNASTAR, Madison, WI). Sequences were generally binned according to taxon of origin, however sequences obtained from the closely related taxa C. vomitoria and Calliphora vicina were very similar to one another and were therefore considered as a single group, hereafter referred to as C.vom/C.vic. Sequences were then aligned using MUSCLE (Edgar 2004; Edgar 2004) and analyzed visually for obvious subgroups within each bin. Clusters of similar sequences containing at least three representatives were used to generate consensus sequences 
(Supplemental Figures 1-5). Modeltest (Posada and Crandall 1998) as implemented in PAUP* v4b10 (Swofford 2002) was used to select the best (of 56) model of DNA sequence evolution for the given sequence clusters (Table 2.1). Base frequencies, substitution models and among site variation rates from Modeltest were then used to calculate maximum likelihood genetic distances between each representative sequence and its corresponding consensus.

Consensus sequences were confirmed as CR1-derived using BlastX (Altschul, 1990) searches of GenBank. BlastX hits were also used to establish the reading frame for sequence translation. Alignment of the translated sequences and several ORF 2 portions of known nonLTR elements (Genbank accession numbers: XP_001193594.1, XP_001949771.1, BAC57926.1, ABH11542.1, BAC57904.1, BAC57908.1, BAC57912.1, CAB99192.1, AAX07241.1, AAZ15237.1, PIR: T25782, AAA53489.1, AAX07243.1, AAQ57129.1, ABR08300.1, ABI15188.1, ABI15187.1, AAC06264.1, XP_001200675.1, AAA49027.1, AAP88020.1, BAA88337.1, AAX07244.1, AAA29354.1, CAJ13354.1, AAB26437.2. Repbase submissions: RTE-1_MD, LINE-1_AA, CR1-1_DWil, CR1-2_HM, RTE-1_AG) was performed using MUSCLE (Edgar 2004; Edgar 2004). Phylogenetic analysis of the aligned translated CR1derived RT portions was accomplished in MEGA(Kumar, Tamura et al. 2004) by performing a neighbor joining analysis using the equal input option and 500 bootstrap replicates. 
Table 2.1 Results of Modeltest analysis.

\begin{tabular}{|c|c|c|c|}
\hline $\begin{array}{l}\text { Grouping } \\
\text { consensus }\end{array}$ & Model chosen & $\begin{array}{l}\text { Proportion of } \\
\text { invariable sites }\end{array}$ & $\begin{array}{l}\text { Gamma distribution } \\
\text { shape parameter }\end{array}$ \\
\hline S. bullata CR1A & TMV & 0.8662 & $\mathrm{~N} / \mathrm{A}-$ Equal distribution \\
\hline S. bullata CR1B & HKY & 0 & N/A - Equal distribution \\
\hline $\begin{array}{l}\text { C. macellaria } \\
\text { CR1A }\end{array}$ & TMV & 0.6074 & N/A - Equal distribution \\
\hline $\begin{array}{l}\text { L. sericata } \\
\text { CR1A1 }\end{array}$ & HYK & 0.9747 & N/A - Equal distribution \\
\hline $\begin{array}{l}\text { L. sericata } \\
\text { CR1A2 }\end{array}$ & K80 & 0 & N/A - Equal distribution \\
\hline L. sericata $\mathrm{CR} 1 \mathrm{~B}$ & F81 & 0 & N/A- Equal distribution \\
\hline $\begin{array}{l}\text { C.vom/C.vic } \\
\text { CR1A }\end{array}$ & HKY & 0 & N/A - Equal distribution \\
\hline $\begin{array}{l}\text { C.vom/C.vic } \\
\text { CR1B1 }\end{array}$ & HKY & 0 & N/A - Equal distribution \\
\hline $\begin{array}{l}\text { C.vom/C.vic } \\
\text { CR1B2 }\end{array}$ & HKY & 0 & N/A - Equal distribution \\
\hline P. regina $\mathrm{CR} 1 \mathrm{~A}$ & HKY & 0.9302 & 0.6242 \\
\hline P. regina $\mathrm{CR} 1 \mathrm{~B}$ & HKY & 0.9587 & N/A - Equal distribution \\
\hline P. regina $\mathrm{CR} 1 \mathrm{C}$ & F81 & 0 & N/A - Equal distribution \\
\hline
\end{tabular}

\section{Results}

PCR analysis produced a single discrete band for each taxon examined. Each amplicon was found to be $\sim 550$ bases (Figure 2.2), which was the expected length. Cloning of each of these bands was successful and sequences from the clones were obtained. Some resulting sequences showed no similarity to at least two other sequences and were therefore excluded from further analysis. The number of analyzed sequences obtained from each taxa and the number of consensus sequences generated from them are shown in Table 2.2. For most taxa (C. macellaria being the lone exception) at least two distinct clusters of sequences were observed. 
Table 2.2 Number of sequences obtained along with number of consensus sequences obtained from them for each examined taxa.

\begin{tabular}{lll}
\hline Taxa & $\begin{array}{l}\text { Number of sequences } \\
\text { obtained }\end{array}$ & $\begin{array}{l}\text { Number of consensus } \\
\text { sequences generated }\end{array}$ \\
\hline Lucilia sericata & 10 & 3 \\
Sarcophaga bullata & 27 & 2 \\
Calliphora vomitoria/vicina & 38 & 3 \\
Phormia regina & 13 & 3 \\
Cochliomyia macellaria & 8 & 1 \\
\hline
\end{tabular}

A BlastX search using the consensus sequence from each grouping resulted in high similarity to known CR1 elements thus confirming the recovered sequences as portions of the RT encoding ORF of a CR1-like element (Table 2.3). The reading frame established from this search revealed no premature stop codons upon translation of the consensus sequences.

Table 2.3 Similarity results as given by BlastX of CR1-like nucleic acid consensus sequence to known CR1 amino acid sequences.

\begin{tabular}{lllll}
\hline Consensus & $\begin{array}{l}\text { Most similar } \\
\text { accession }\end{array}$ & Organism & $\begin{array}{l}\text { E } \\
\text { value }\end{array}$ & $\begin{array}{l}\text { Bit } \\
\text { Score }\end{array}$ \\
\hline S. bullata CR1A & CAB99192.1 & D. melanogaster & 2e-44 & 181 \\
S. bullata CR1B & XP_001950444.1 & A. pisum & 2e-35 & 152 \\
C. macellaria CR1A & AAK39265.1 & C. elegans & 2e-24 & 115 \\
L. sericata CR1A1 & CAB21860.1 & T. brucei & 2e-08 & 62 \\
L. sericata CR1A2 & AAK39265.1 & C. elegans & 2e-30 & 135 \\
L. sericata CR1B & CAB99192.1 & D. melanogaster & $9 \mathrm{e}-51$ & 202 \\
C.vom/C.vic CR1A & CAB99192.1 & D. melanogaster & 2e-39 & 165 \\
C.vom/C.vic CR1B1 & ABI15187.1 & M. nausithous & $3 \mathrm{e}-49$ & 197 \\
C.vom/C.vic CR1B2 & ABI15187.1 & M. nausithous & $9 \mathrm{e}-50$ & 199 \\
P. regina CR1A & CAB99192.1 & D. melanogaster & $4 \mathrm{e}-43$ & 177 \\
P. regina CR1B & BAC57910.1 & A. gambiae & $3 \mathrm{e}-45$ & 184 \\
P. regina CR1C & AAZ15237.1 & A. aegypti & $1 \mathrm{e}-33$ & 146 \\
\hline
\end{tabular}

Results of the phylogenetic analysis of the translated amino acid sequences are presented in Figure 2.3. An inclusive carrion fly cluster was not observed when compared to CR1 elements from a wide variety of outgroup taxa. A subset of carrion fly consensus sequences was found to form a monophyletic cluster with R1-like elements 
such as the Pilger element from D. melanogaster. S. bullata CR1A falls out separately from the carrion fly R1-like elements and forms a cluster with various elements from $A$. gambiae. However, there is little support for excluding the CR1A consensus derived from S. bullata from carrion fly elements in the R1-like clade, (i.e. those recovered from $L$. sericata, C. vomitoria, C. vicina, and two elements from P. regina). Overall support for their classification as members of the R1 family of CR1-like elements is therefore high.

\section{C. macelleria CR1A along with $L$. sericata CR1A1 and L. sericata CR1A2} formed a cluster which then formed a larger cluster with Jockey-like elements from a variety of insects but there is little bootstrap support for this association. On the other hand, support for their own cluster is high, suggesting a common ancestral element. $P$. regina CR1C and a CR1-like element from A. aegypti are strongly clustered.

C.vom/C.vic CR1B1 and C.vom/C.vic CR1B2 appear to be members of the Zenon family of elements based on the strong support found for the cluster including elements from butterflies (Shijimiaeoides divina and Maculinea spp.) and moths (Bombyx mori). It is interesting that an element from a very distantly related organism, the cnidarian Hydra magnipapillata, is also a member of this cluster.

Finally, the consensus S. bullata CR1B finds itself as part of a cluster that includes elements from the taxon from which the classical CR1 element originally described, the chicken (Gallus gallus). The clade includes elements occupying the genomes of vertebrates (Gallus, Chelonia and Platemys), blood flukes (Schistosoma), and sea urchins (Strongylocentrotus) among others. However, the element from S. bullata is clearly most strongly associated with a CR1 from a fellow dipteran Drosophila willistoni. 


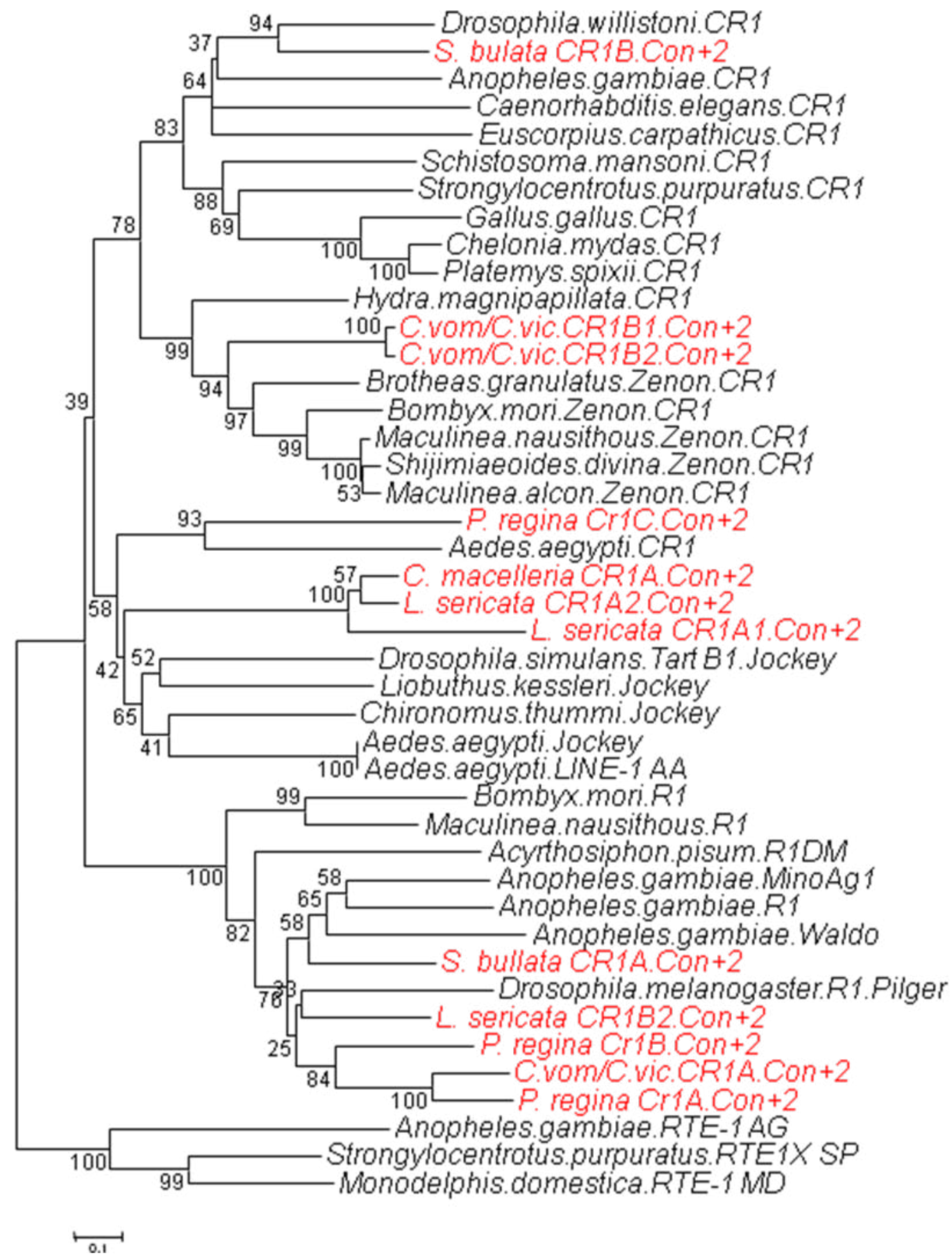

Figure 2.3 Phylogenetic tree inferred from translated carrion fly consensus sequences and several outgroups comprising known CR1-like elements. Carrion fly taxa are highlighted in red. The +2 indicates the reading frame for recovered sequences when compared to the original consensus sequence. 
Analysis of divergence values within taxa revealed that there is little diversity within each presumed subfamily in several lineages (Table 2.4). When comparing each respective sequence to the consensus sequence, all $P$. regina clusters (CR1A, CR1B and CR1C) and the L. sericata CR1A1 cluster revealed over 99\% sequence identities. The clusters C.vom/C.vic CR1A, L. sericata CR1B and S. bullata CR1B also showed high sequence identities (above 98\%). Standard deviation values also show there is little difference between sequences residing in the same cluster.

Table 2.4: Divergence and standard deviation values calculated for each cluster of elements.

\begin{tabular}{lll}
\hline Consensus & $\begin{array}{l}\text { Average divergence } \\
\text { value }\end{array}$ & Standard Deviation \\
\hline P. regina CR1A & 0.005282 & 0.002767 \\
P. regina CR1B & 0.006222 & 0.007761 \\
P. regina CR1C & 0.006908 & 0.011965 \\
C.vom/C.vic CR1A & 0.012033 & 0.021723 \\
C.vom/C.vic CR1B1 & 0.030555 & 0.011378 \\
C.vom/C.vic CR1B2 & 0.029534 & 0.021355 \\
S. bullata CR1A & 0.030447 & 0.016793 \\
S. bullata CR1B & 0.015637 & 0.022969 \\
L. sericata CR1A1 & 0.003882 & 0.002814 \\
L. sericata CR1A2 & 0.029915 & 0.002005 \\
L. sericata CR1B & 0.012513 & 0.009294 \\
C. macelleria CR1A & 0.024084 & 0.022988 \\
\hline
\end{tabular}

\section{Discussion}

While in mammals, one typically finds only a single lineage of active non-LTR retrotransposons, having multiple lineages of active non-LTR elements is not uncommon in non-mammalian animal taxa (Furano, Duvernell et al. 2004). For example, 21 families of non-LTR retrotransposons were found to have been recently active in the genome of the African malaria mosquito, Anopheles gambiae (Biedler and Tu 2003). While not as extreme an example, most of the carrion fly families examined here also appear to harbor multiple recently active non-LTR retrotransposons. Each examined species shows 
evidence of at least one lineage of CR1-like elements present in their genome and in many taxa, more than one lineage of elements is apparent.

Comparison of each representative sequence to its respective consensus sequence revealed high sequence identities suggesting some of these elements have been recently active. This is indicative of the master gene model where the elements are likely the result of recent retrotransposition from a common source. This is especially true for the clusters that showed above a 98\% sequence identity. It is unfortunate that neutral mutation rate data for the nuclear genome is unavailable for carrion flies. Such information would allow us to attach possible time frames to the divergence values we observed. Additional evidence of recent activity is the lack of any unexpected stop codons or other mutations that might suggest that they are nonfunctional elements.

One example is the S. bullata CR1B cluster. It exhibited one of the lowest divergence values between its representative amplicons and the consensus. Another example is found within $P$. regina where all three clusters (CR1A, CR1B and CR1C) showed over a 99\% sequence identity. Though these exhibit such a high similarity within the cluster, the phylogenetic analysis reveals they show little similarity between clusters. This supports the hypothesis that there are separate lineages that have propagated throughout the genome. C.vom/C.vic CR1A with eight representative sequences forming the cluster retained over $98 \%$ sequence identity suggesting it too is recently active. All other families recovered showed at least $96 \%$ sequence similarity suggesting they have been active relatively recently, but may not be as young as the other families.

The observation that numerous recently active families of CR1-like elements are present in representative carrion flies suggests that we will eventually be able to identify 
taxon-specific insertion events that will be useful for species identification at a later date. This will enable the eventual development of a TE-based method for species identification that will be of particular use in determining taxon identity for specimens collected at crime scenes. Additional applications are also possible for phylogenetic analyses of these complex taxonomic assemblages.

Further research will be done to characterize the elements identified here. This research will begin with recovering the complete ORF2 sequence from the $S$. bullata CR1B cluster. We chose the CR1B lineage from S. bullata because it consisted of the largest amount of sequences used to create the consensus, and showed one of the lowest divergence values. This suggested that intact copies of the master element are likely still present in the genome. Moreover, when compared to the known non-LTR elements it was the one of only three consensus clusters to form in the clade of CR1 elements with a high bootstrap value. 
Chapter 3: Characterization of CR1 element identified within Sarcophagidae 


\begin{abstract}
The goal of this study was to characterize the S. bullata CR1B element identified in the previous chapter though further sequencing in both the 5' and 3' direction. This was accomplished using a genome walk begun from the original consensus sequence. The final constructed sequence is presumed to be the complete Open Reading Frame 2 (ORF2) portion of the element containing both the reverse transcriptase (RT) and endonuclease (EN) domains. These domains were used to identify conserved amino acid regions in the recovered consensus via comparison to known non-LTR retrotransposons. A phylogenetic analysis of the full RT domain revealed the recovered ORF in S. bullata also compares favorably with previously documented CR1-like elements.
\end{abstract}

\title{
Introduction
}

Non-LTR retrotransposons have variable structures, but usually contain one or two open reading frames (ORFs). The function of ORF1 in non-LTR retrotransposons that contain it remains questionable, in part due to their absence in some non-LTR elements. Conserved domains have been identified in what ORF1 representatives we have. LINE 1 (L1) elements reveal there is a high probability of a coiled-coil domain along with nucleic acid binding domain being encoded within ORF1 suggesting a singlestranded binding function (Martin, Li et al. 2000). A zinc finger is proposed to be encoded in ORF 1 of both I and Jockey elements (Martin 2006). Also, an esterase domain (ES) or plant homeodomain (PHD) has been identified in ORF1 of CR1 elements in a variety of taxa (Kapitonov and Jurka 2003).

Though the function of ORF 1 remains inconclusive, ORF2 is well known to encode enzymes essential for retrotransposition. ORF2 has been shown to encode 
enzymes with combined endonuclease/ reverse transcriptase (EN/RT) activity. While clearly distinct, CR1 elements are clustered with the L1 family of elements based on the reverse transcriptase encoded within ORF2. What separates them from the L1 elements is the differing protein sequences also encoded within the ORF2.

Phylogenetic analysis of the reverse transcriptase portion of non-LTR elements has enabled differentiation among classes of elements. This is often done with focus on the finger/palm region which corresponds to the amino terminal half of the RT domain of HIV (Unge, Knight et al. 1994). Eleven clades of non-LTR elements have been found based on analysis of the entire RT domain: Jockey, Chicken Repeat 1 (CR1), LINE 1 (L1), CRE, I, R1, R2, R4, RTE, Tad and LOA (Malik, Burke et al. 1999). As seen in Figure 3.1, the combined EN/RT coding region comprises a majority of several average complete elements (Tad, R1, LOA, I, Jockey and CR1).

Though the full element is much larger, a vast number of copies of CR1 elements within the chicken genome, where the element was first discovered, and in other taxa, are shorter than 400bp (ICGSC 2004). This is most likely due to truncation of the 5' element end during mobility (Haas, Grabowski et al. 2001).

Though the 5' end of the element is often truncated the 3' end shows characteristic features. The Drosophila CR1-like elements contain a specific sequential repeat on the 3' element end that is often followed or preceded by a polyadenylation signal (Kapitonov and Jurka 2003). This eight base pair direct repeat region is yet another character distinguishing CR1-family elements from the rest of the L1 elements which simply have a poly-A tail. 


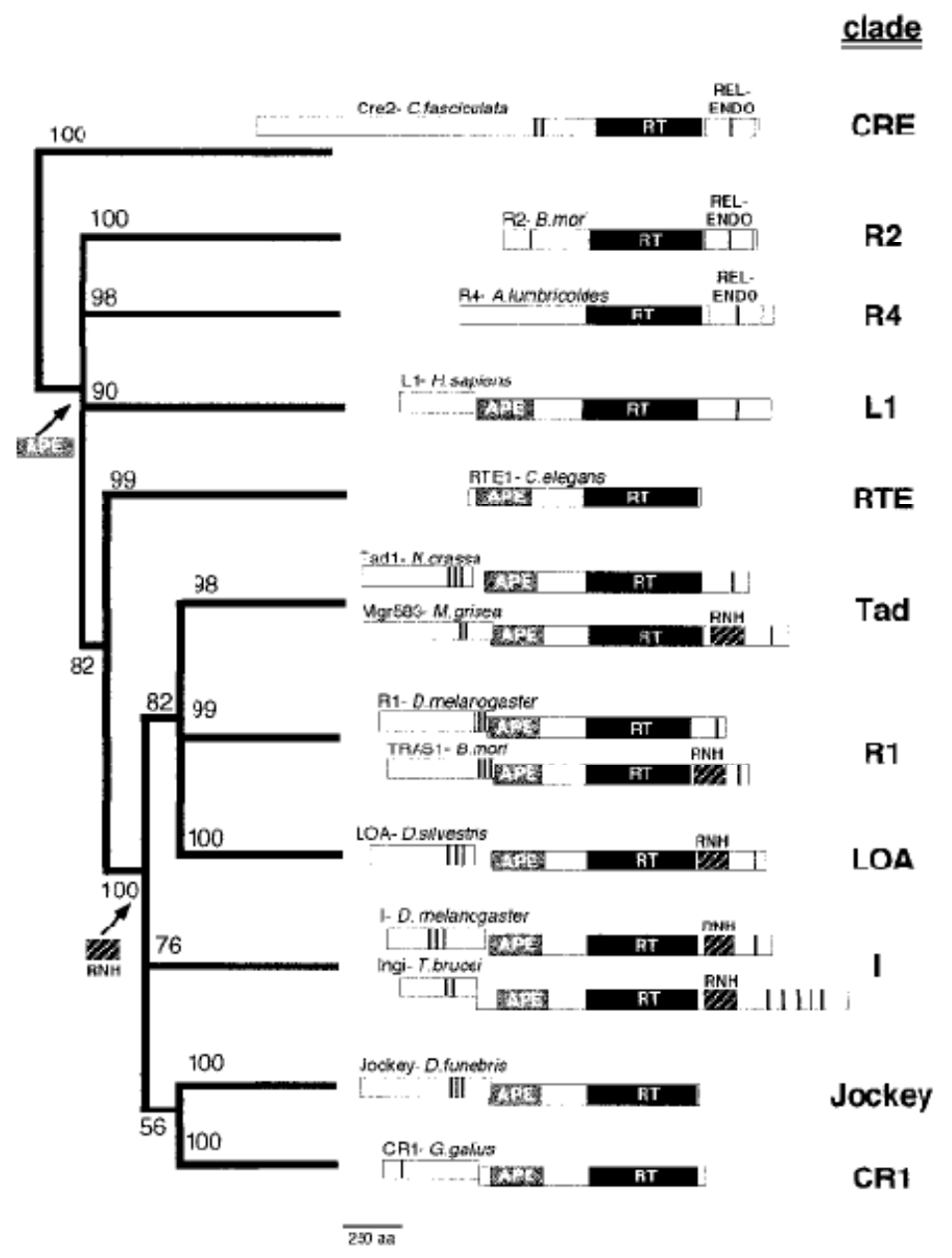

Figure 3.1: A neighbor joining tree of 11 major clades of non-LTR elements inferred from the reverse transcriptase domains encoded by them (Malik, Burke et al. 1999). Enzymatic domains encoded in each element (RT -reverse transcriptase, APE -apurinic/apyrimidinic endonuclease, RELendo - restriction like endonuclease and RNH - RNase H domain) are shown in the shaded boxes. Vertical bars indicate cysteine-histidine motifs.

The purpose of this research is to characterize the EN/RT domain of a CR1-like element from Sarcophaga bullata. This will aid in determining positive identification for the element and allow the development of better amplification primers for additional analysis in other closely related taxa. Amplification in other taxa will further cement the groundwork of TEs as a maker system for species identification in forensically important carrion flies. 


\section{Materials and Methods}

DNA was extracted from the thoracic tissue of Sarcophaga bullata using the Qiagen Puregene Tissue Core Kit A (Gentra, Minneapolis MN) and diluted to 0.1ug/ul. The manufacturer's protocol provided in the GenomeWalker Universal Kit (Clontech, Mountain View CA), was followed to digest each of four aliquots of genomic DNA with one of four restriction enzymes (Dra I, EcoR V, Pvu II and Stu I). Resulting fragments larger than 1,000 bp were cleaved from a 1\% sodium borate agarose gel, cleaned with the Wizard SV gel and PCR clean up system (Promega, Madison WI) and linkers from the GenomeWalker Universal kit were ligated using the manufacturer’s protocol.

Two consecutive primers were designed, round 1 Right Out (RO) and round 1 RO nested to amplify the 3' direction and two primers, round 1 Left Out (LO) and round 1 LO nested were developed for the 5' direction using the partial CR1 original consensus sequence of $S$. bullata CR1B described in Chapter 2 (Figure 3.2). They were used along with the GenomeWalker Universal Kit linker primers to obtain several amplicons $>1 \mathrm{~kb}$ which were visualized in a $1 \%$ sodium borate gel, cleaved from that gel, and purified using the Wizard SV gel and PCR clean up system (Promega, Madison WI). The resulting fragments were ligated into the TOPO TA (Invitrogen, Carlsbad CA) vector using the manufacturer's protocol, transformed into Top10 cells and grown on $\mathrm{LB} /$ Kanamycin $(2 \mu \mathrm{l} / \mathrm{ml})$ plates overnight. Individual colonies were grown in LB/Kanamycin $(2 \mu \mathrm{l} / \mathrm{ml})$ broth overnight and plasmids were isolated using Fast Plasmid Mini kit (Eppendorf, Hamburg GER). Purified plasmids were sequenced using Big Dye Termination sequencing on an ABI 3100 Genetic Analyzer. 


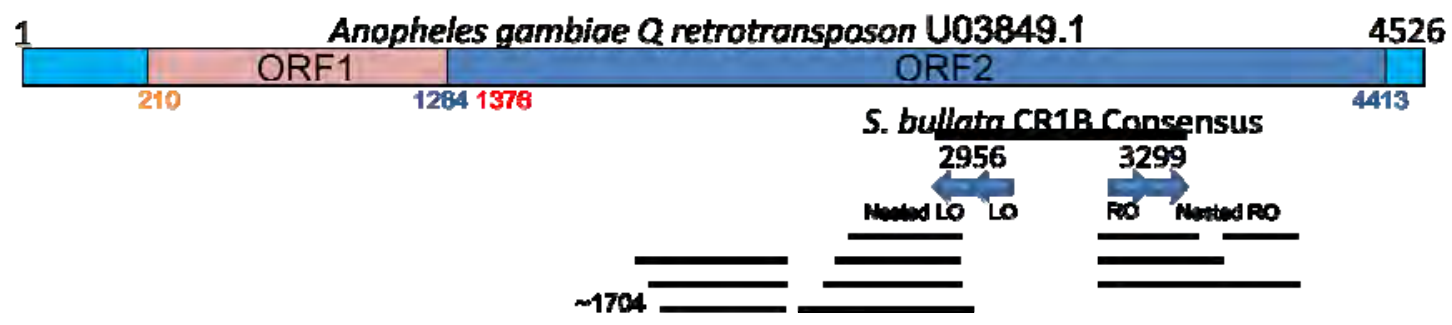

Figure 3.2 Original primer pairs (blue arrows) developed for both the 5' and 3' direction of the element. Numbers indicate position of the base pairs relative to the full element. Black lines below the primers indicate the resultant fragments and their relative positions inferred after sequence analysis. These sequences were used to develop new primers for additional genome walk rounds to expand our analysis of the CR1-like element.

Vector sequence was removed from the resulting sequence data using SeqMan (DNASTAR, Madison, WI). Sequences were confirmed as CR1-derived using Blastn and BlastX (Altschul, 1990) searches of GenBank. The recovered first round sequence consensus showed high similarity to the CR1 element from Anopheles gambiae (accession number AAA53489.1). We used this sequence as a reference for the remainder of the genome walk.

The genome walk was then continued for several rounds with two new primers in each direction. Primers sequences for each round are described in Table 3.1. Occasional gaps and ambiguities in the sequence data were seen, usually because sequence data (averaging 600 bp) for larger fragments (some larger than $1300 \mathrm{bp}$ ) was insufficient to obtain the entire fragment. In order to remedy this, additional primers were developed to amplify these regions and are denoted by half rounds (e.g. round 3.5) in Table 3.1.

At the 5' end, what appeared to be the end of the ORF was reached as evidenced by a lack of sequence similarity in any cloned fragments beyond this point. A similar observation was used to identify the 3' end. 
Sequences from each round of sequencing were aligned and a complete contig sequence consensus was constructed (Supplemental Figure 6). The resulting consensus sequence was entered into the NCBI ORF finder (http://www.ncbi.nlm.nih.gov/gorf/gorf.html) and one large ORF was identified. Querying Blast (BlastX) with the sequence revealed high similarity to several RT/EN encoding ORFs. Several of these were extracted and MUSCLE (Edgar 2004; Edgar 2004) was used to align the recovered contig consensus S. bullata sequence with these known retroelement RT (Accession numbers: AAC72810.1, AAC51279.1, AAC72298.1, AAA21781.1, AAC13649.1, AAB92389.1, AAA28675.1, AAA29354.1, AAA28291.1, AAA53489.1, AAC06263.1, AAA49027.1, and CAA29181.1) and EN domains. Shading of similarity was done using BioEdit graphic view option. All sequences generated for this work have been deposited in GenBank under accession numbers FJ941020 through FJ941066.

Phylogenetic analysis of the RT domain from the translated S. bullata contig consensus and known CR1-like non-LTR elements (Accession numbers: BAA88337.1, AAC60281.1, U66331.1, AAR31731.1, AAA53489.1, AAZ15240.1, AAD19348.1, CAA29181.1, CAB41693.1, AAB65093.1, AAB92389.1, AAA21781.1, AAC72298.1, AAA29363.1, AAC13649.1, AAA28675.1, AAB04627.1, AAA29354.1, AAA28291.1, ABH11541.1, ABI15177.1, AAC72810.1, and AAC51279.1) was accomplished by performing a neighbor joining analysis using the equal input option and 500 bootstrap replicates. 
Table 3.1: Sequence of primers used in conjunction with the GenomeWalker Universal Kit protocol to collect walk out data for each round.

\begin{tabular}{lll}
\hline Primer Name & Sequence (5’-3') & $\begin{array}{c}\text { 5’ Position in } \\
\text { final alignment }\end{array}$ \\
\hline Round 1 RO & CTTACTCGTTCATAAGCTTTATCAAC & 2260 \\
$\begin{array}{l}\text { Round1 Nested } \\
\text { RO }\end{array}$ & GCAGTGCGTTAAATTCAGGAACACA & 2340 \\
Round 2 RO & CCCAATTCTTGAATATGGATCTGTAA & 2857 \\
Round 2 & GTGGTTTACAATGGGATCCTAATGT & 2959 \\
Nested RO & & \\
$\begin{array}{l}\text { Round 1 } \\
\text { Nested LO }\end{array}$ & GGAGGGAAAATGGCCCAATTTAAGTGATTTG & 1955 \\
Round 1 LO & GAGATATCAGCTTTAGATCCCGATT & 1982 \\
$\begin{array}{l}\text { Round 1.5 } \\
\text { Forward }\end{array}$ & CCTGAAGACAAATATCATCCGACAC & 1124 \\
$\begin{array}{l}\text { Round 1.5 } \\
\text { Reverse }\end{array}$ & GGAATACAATCAACGAAACAATCGT & 1341 \\
Round 2 LO & CTAATACGCATATCAACTCAATATG & 734 \\
Round 2 & GAAGGAAACTTCTTCAGACGAGAA & 698 \\
Nested LO & & \\
Round 3 LO & CTACTACTGAGCCAAGTTTCAGTG & 553 \\
$\begin{array}{l}\text { Round 3 } \\
\text { Nested LO }\end{array}$ & GAAGATAATTTCGAGTTAATCCCTCT & 507 \\
$\begin{array}{l}\text { Round 3.5 } \\
\text { Right In }\end{array}$ & CCTGTTCCATATACCTTAATC & \\
\hline
\end{tabular}




\section{Results:}

The final contig consensus sequence built from all genome walk data was just over 3,400 bases. When translated in the +2 frame just over 800 amino acid residues were obtained to produce a $\sim 90 \mathrm{kD}$ protein. This translation was compared to known non-LTR retrotransposons ORFs and found to be of average length.

We found several highly conserved regions common to the reconstructed $S$. bullata CR1 ORF contig consensus sequence and previously confirmed CR1 ORF sequences they are shown in Figure 3.3. Previous analyses had established eight conserved regions, located in the Fingers/Palm region, and three in the thumb region across all non-LTRs (Xiong and Eickbush 1990; Malik, Burke et al. 1999).

Similarity to known non-LTR retrotransposons was also assessed in the EN domain. The S. bullata consensus sequence is shown alongside known endonuclease domains (Figure 3.5). Areas shaded in black are identical amino acids while areas shaded in grey show greater than $80 \%$ similarity within the alignment. There are several highly conserved regions seen in this alignment indicating the element portion from S. bullata likely codes for endonuclease activity.

Representative sequences from eight of the non-LTR element groups were used to infer a phylogeny of known RT domains including the $S$. bullata presumed RT domain. For the sake of brevity seven non-LTR elements most closely related to CR1 were chosen for this analysis. As Figure 3.4 shows, the S. bullata CR1 presumed RT domain falls into the 'classical' CR1 clade with 77\% bootstrap support. This result reinforces results from the previous chapter using a smaller fragment from the same RT domain. We also see that the S. bullata CR1-like sequence clusters with known CR1-like elements from two 
other dipterans, the mosquitoes Anopheles gambiae and Aedes aegypti with relatively high support values.

Specific amino acid residue polymorphisms between the CR1 clade and other

element clades were identified. For example Tyrosine and Aspartate residues were found to be consistent among the CR1-like elements only at residue numbers 314 and 333 respectively. 


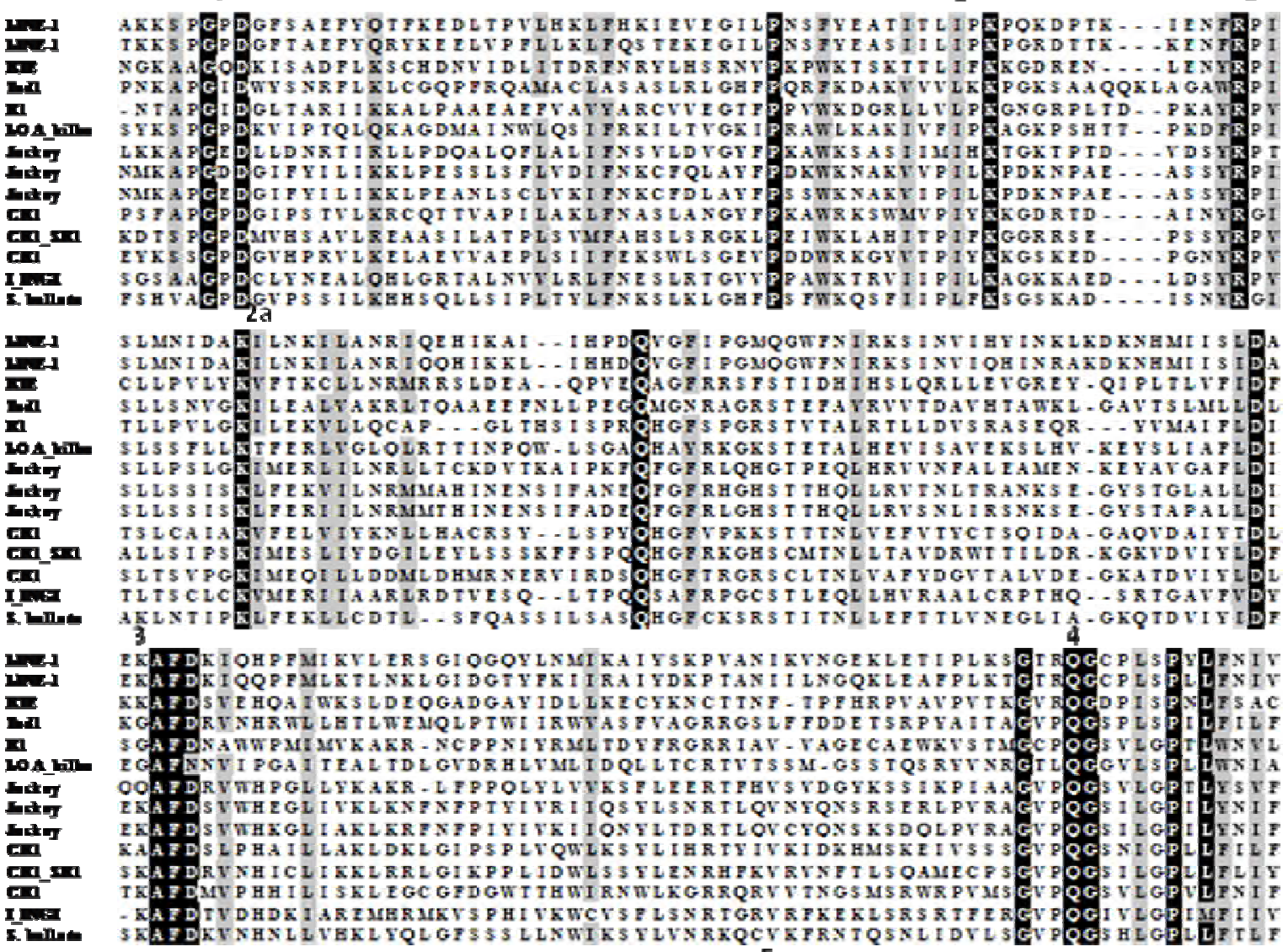

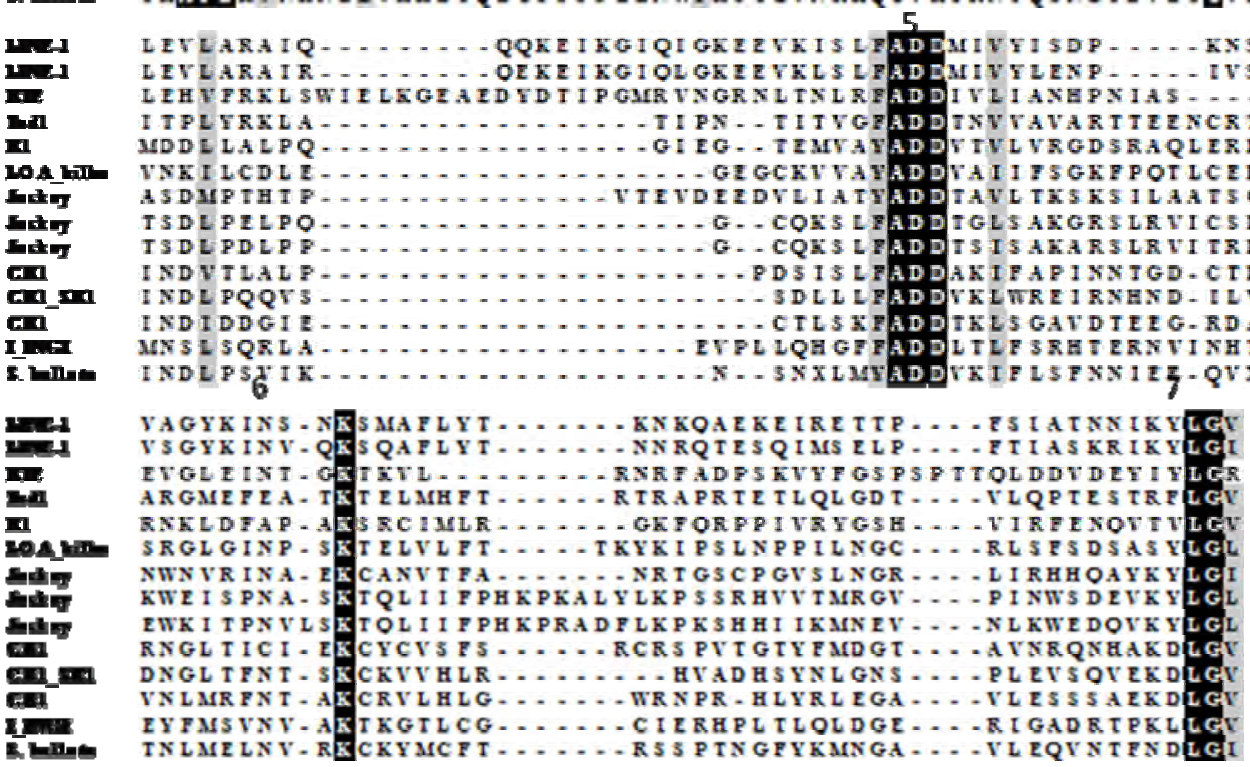

Figure 3.3 Alignment of S. bullata CR1B ORF2 with sequences of known non-LTR RT Fingers/palm region and one in the thumb region (area 7). Areas shaded in black are identical while areas shaded in grey show $80 \%$ similarity. Numbers above sequence denote the seven highly conserved areas previously described. 


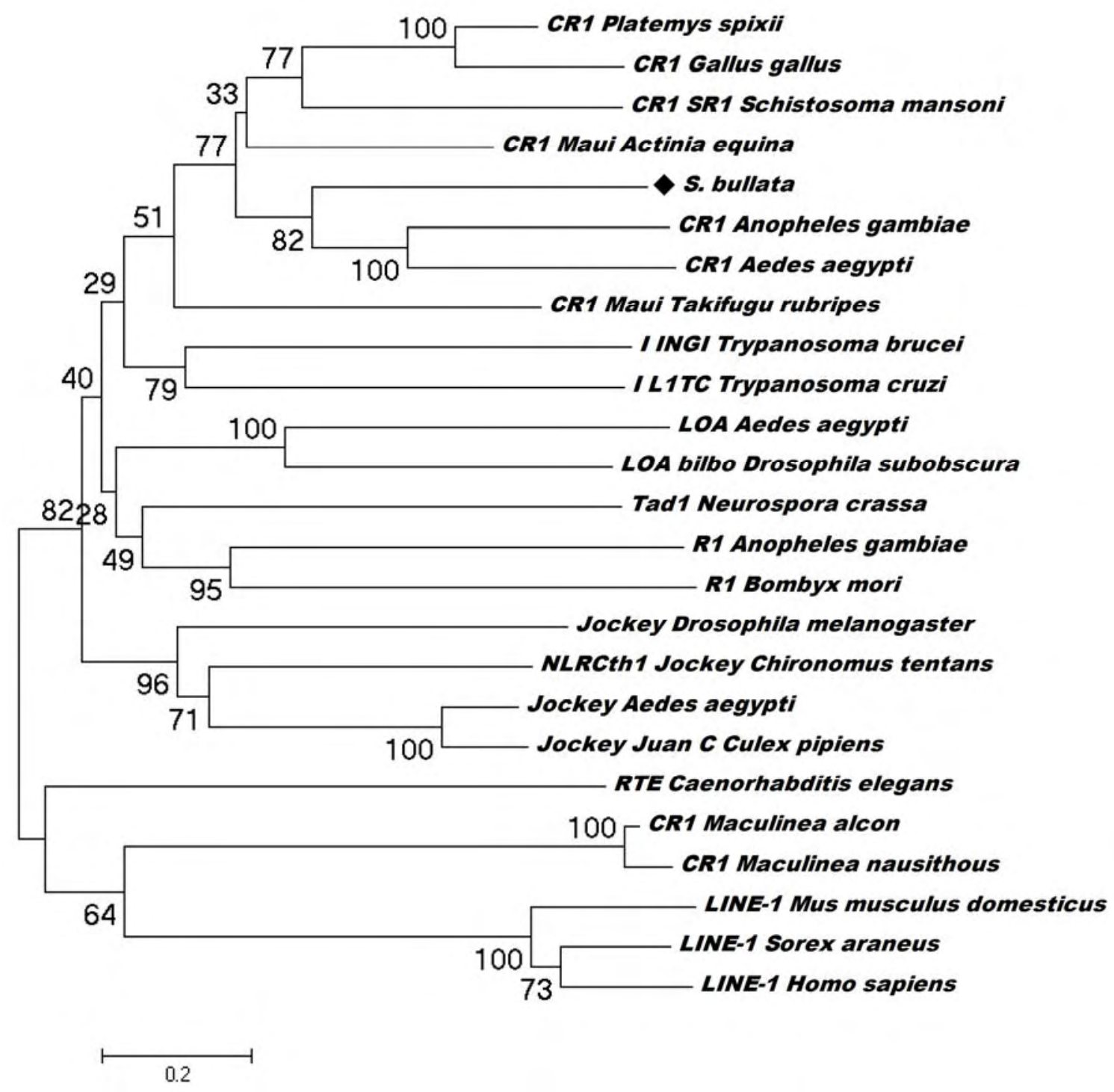

Figure 3.4 Results of a neighbor joining phylogenetic analysis of the RT domain of CR1-like nonLTR elements with the RT portion of the recovered element from $S$. bullata. Values on nodes are the proportion of bootstrap replicates supporting that node. 
XP_001181139.1 [S. purpuratus] AAL 40415.1 [B. floridae] BAA88337.1 [P. spixii]

XP_002157920.1 [H. magnipapil] XP-001195308.1 [S. purpuratus] CĀJ00235.1 [S. mansoni] CAJ00242.1 [S. mansoni] CAJ00238.1 [S. mansoni] CAJO0244.1 [S. mansoni] DAA04497.1 [S. mansoni] T25005 [C. elegans] AAZ15238.1 [C. briggsae] B34751 [A. gambiae] S. bullata

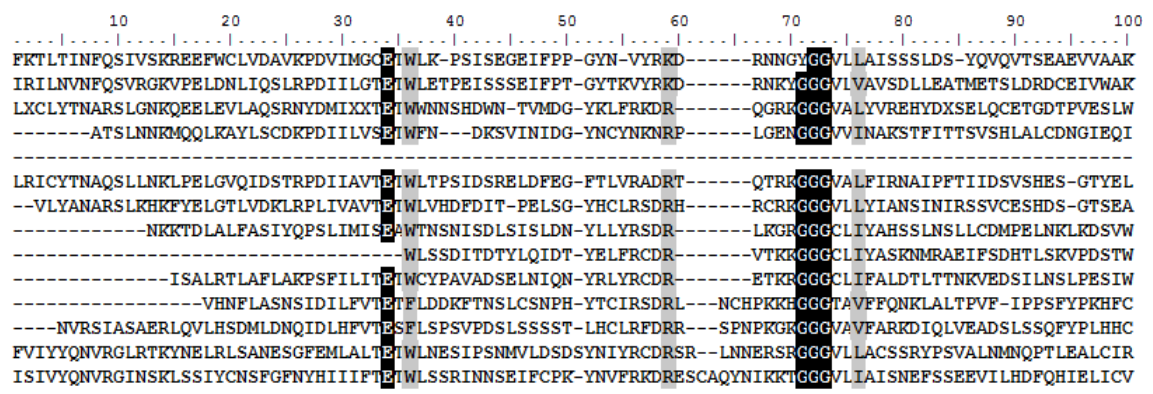

XP 001181139.1 [S. purpuratus] AALA 40415.1 [B. floridae] BAA88337.1 [P. spixii] XP_002157920.1 [H. magnipapil] XP_001195308.1 [S. purpuratus] CAJU0235.1 [S. mansoni] CANO0242.1 [S. mansoni] CAJ 00238.1 [S. mansoni] CAJ00244.1 [S. mansoni] DAA04497.1 [S. mansoni] T25005 [C. elegans] AAZ15238.1 [C. briggsae] B34751 [A. gambiae] S. bullata

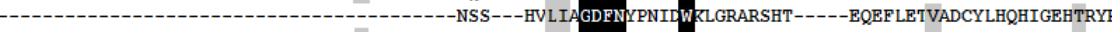
ISTN-TLNHS-LLLG-CIYRAPDSTDNLNDRITN-AFIHASTLNESARI
XP 001181139.1 [S. purpuratus] AAL 40415.1 [B. floridae] BAA88337.1 [P. spixii] XP_002157920.1 [H. magnipapil XP-001195308.1 [S. purpuratus] CA.JO0235.1 [S. mansoni] CAJO0242.1 [S. mansoni] CAJO0238.1 [S. mansoni] CAJO0244.1 [S. mansoni] DAA04497.1 [S. mansoni] T25005 [C. elegans] AAZ15238.1 [C. briggsae] B34751 [A. gambiae] S. bullata

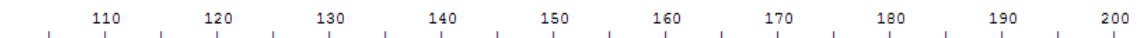
IVNRNNNNS--VIFG--AVYRPPNTNQPYMDNMNQFIRELCMSNPGTALCIG GD M NLPIDWETEEIRSHQYRKSLSETYLQTLADVGLSQIVNFPTRGS VKLRGKRD---LLIG--SYYRPHEGLQES LKFMAESVRLACQSR-NAIVVLG GDFNLPDWLWKEKVLKPGSSYPNI HRQF IDIISDLGMEQIVEKP TRGE VKLRSKNNKGDVVVG--VCYRXPDQEDEVDEAFF SQLGKVSSSQ---DLILYGDFNHPDICWKSNTAEHKQ-----SRKF LXXVADNFLVQVLEQPTRGR WCSLTNGPDHLLIGCIYRPPSSPESVNTKINSVLGIAKSLVDQKKYTGLIITT VSCRLKCRGELLLS--LIYR--SPSCEANEVLLNS LNT LSRSD---RCLI LGDFNAPMVDWGNLRTESSANSF--EQELVDAVITCALVQHVKEATRYD ISCELTVGCCTFALG--VIYR--SPICLADDF I LEHIRLWSANN---RCLILGDFNAPDISWTEMTTKCS INSF--DSRLLETVMEHALVQHVSKP TRFG IVLKPSNSIT-LLFG--CIYQQPN-LAIGKIAVLSEVFT LASSLPFTGKLICGDFNMPEI WFPVVAPKRY------ESFIECLELGQWTQYVSSPTRHQ LTVH-TLECK-ILIG--CIYRAPN-TPSLTDTIISESFAKAASLDFACKVVCGDFNLPTINWKTHSGPPCY------EGILRSLDIHGWDQHVNLPTRN EILALDHKPSKTRF I--LTYRPPS IAVRRT SE LFNNLNHLIDIPTYSF-VLLGDFNFANTHW WSNNLPLN-------SEYLFNhMIKFHNLVQLVNFPSRFT EIITVDLLISCRFV--LVYCPPSTSPAQSDVLFHNLSLLLSN-SYRFNFLLGDFNYPQINWSSDNLLPNDL----PAGLESLMSLSDLTQIVDFPTRTS VSFPKFRLYVGIVYVPPYLSSDRNYFESLSAF IXDAYMHMIKPND---HLILL LVKI HNQSLFIVCVY----IPPNSPLTVYEDYLNAIDTLLRKVNTTDLVLLIGDFNMSHTSWKAVXDSNYLIPVSSSESYANIINCLLESCPFQINSIPN

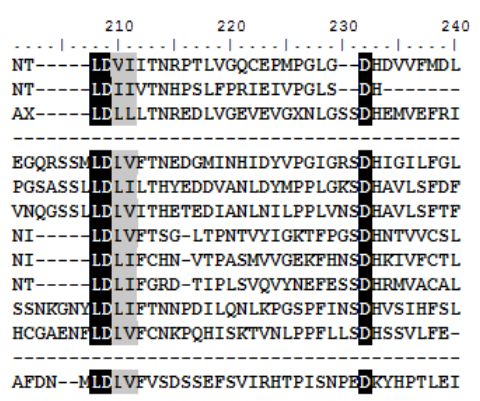

Figure 3.5 Sequence alignment showing similarity of a portion from $S$. bullata to known endonuclease domains. Accession numbers and species of origin are shown alongside each sequence.

\section{Discussion}

The S. bullata contig consensus sequence shares certain highly conserved regions with all non-LTR elements. The RT domain reconstructed in this work contains all seven highly conserved regions in the finger/palm region of typical non-LTR RT domains.

These domains allow for classification of this element. For instance the $S$. bullata contig consensus sequence contains area 0 , termed the $\mathrm{Z}$ domain, which is found to be only consistently found in retrotransposons (McClure 1991). The best conservation across all element types is found in areas two and four. 
Separation of the S. bullata CR1 sequence from the R2, R4, and CRE-like element families is clear due to the presence of an AP endounculease (APE) in the sequence where these families do not contain this domain (Malik, Burke et al. 1999). All of the other element families contain both the RT and APE domains and with the exception of a few I elements are all lacking the RNase H domain (Eickbush and Malik 2002). Further element family separation is indicated by at least $20 \%$ sequence divergence in the RT domain (Biedler and Tu 2003).

The RT regions recovered from this work show high similarity to known CR1 from a wide variety of taxa including reptiles, birds, insects, echinoderms, humans, rodents, protests, worms, anemones and fungi indicating it is likely a 'classical' CR1 element.

CR1 elements were first described in the chicken, Gallus gallus, and were originally thought to be a type of SINE because of their tendency to be truncated at the 5' end (van het Schip, Samallo et al. 1987). We don't know yet if CR1 elements in carrion flies suffer this same fate. However, given that they share the same basic structure and well-conserved amino acid motifs, presumably necessary for retrotransposition, as the classical CR1 elements, it is likely to be the case. Additional genome level data will be required before this can be determined.

It is clear that the RT and EN domains of the S. bullata CR1-like element are present and correspond well with similar elements found in other taxa. The phylogenetic analysis revealed the RT domain formed a cluster with other dipterian CR1s, suggesting that this element has been transferred to the genome vertically rather than horizontally as is often seen for non-LTR retrotransposons. This is not surprising due the low instances 
of observed horizontal transfer of non-LTR elements (Eickbush and Malik 2002). Moreover, many non-LTRs previously believed to replicate through horizontal transfer are now described through phylogenetic incongruence to indeed be replicated through vertical transfer (Eickbush and Malik 2002).

Overall the S. bullata CR1-like element is very similar to the 'classical' CR1 elements and shows signs of functionality as a marker for species identification due to its transmission. It has likely retained its ability to propagate through the genome and therefore intact copies of the master element are still present in the genome. 
Chapter 4: Conclusions 
This study shows the first documentation of LINEs within carrion flies. CR1-like elements were identified in several taxa and one very interesting family S. bullata CR1B, was investigated further. Briefly, the three main observations were made in the course of this study; 1) that carrion flies often harbor several CR1-like lineages, 2) that these lineages often exhibit signs of recent activity and, 3) that highly conserved regions typical of classical CR1-like elements are found in S. bullata CR1B. Below, these major findings will be elaborated. Additionally, extensions and implications of the work will be addressed.

Each species examined with the exception of $C$. macelleria contains several distinct clusters of CR1-like elements. These clusters were often found to show high sequence identities and therefore low divergence values suggesting that they were recently active. Several clusters (P. regina CR1A, P. regina CR1B, P. regina CR1C, C.vom/C.vic CR1A and S. bullata CR1B) showed 99\% sequence identity or higher.

Comparison of the recovered sequence portion of carrion fly element families to known CR1-like element sequences revealed an exclusive carrion fly cluster was not present. This is most likely due to the variety of CR1-like lineages present in the examined species. A subset of carrion fly element clusters from S. bullata, L. sericata, C. vomitoria, C. vicina and P. regina are likely from the $\mathrm{R} 1$ family of elements due to their formation of a cluster with these elements. Another subset of elements from $L$. sericata and the only element from C. macelleria formed a cluster with Jockey-like elements from a variety of insects with little bootstrap support indicating they are likely from the Jockey element family. The Zenon family is likely the classification of the remaining two C.vom/C.vic clusters due to their cluster formation. 
S. bullata CR1B, was found to cluster with, and therefore likely be from the CR1 family of elements. The closest association was with a fellow dipteran then further clustered with other CR1 elements from a wide variety of taxa including vertebrates, blood flukes, and sea urchins. This cluster also showed one of the lowest divergence values (0.015637) likely indicating recent activity. Due to these observations this cluster was chosen for further analysis.

Further sequencing of this cluster revealed several highly conserved regions in comparison to known CR1-like ORF2s. This ORF is known to contain the RT and EN domains which were both identified with high conservation. The RT domain has previously been used to characterize non-LTR element families (Malik, Burke et al. 1999) Phylogenetic analysis of the presumed RT domain revealed for this element showed this element falls into the 'classical' CR1 clade with fellow dipterans the mosquitoes Anopheles gambiae and Aedes aegypti.

The signs of recent activity suggest that we will eventually be able to identify taxon-specific insertion events that will be useful for species identification at a later date. This will enable the eventual development of a TE-based method for species identification that will be of particular use in determining taxon identity for specimens collected at crime scenes.

The need for a reliable identification method for species has become apparent for additional purposes. One such example is the need to correctly identify specific carrion flies for use in wound cleaning by the medical community. Though the COI gene has been investigated for this purpose (Williams, Cronje et al. 2008), this analysis method poses another identification possibility. 
Applications are also possible for phylogenetic analyses of these complex taxonomic assemblages. For example the phylogeny of the carrion fly genus Protocalliphora is not completely resolved due to the large number of species (Sabrosky, Bennett et al. 1989).

Creating transgenic animals with the use of TEs has also become an active area of interest. For example, Class 2 elements known as piggyBacs have been used to investigate genetic modification in the tobacco budworm (Ren, Han et al. 2006) sheep carrion fly, Lucilia cuprina (Heinrich, Li et al. 2002) and the New World screwworm, Cochliomyia hominivorax (Allen, Handler et al. 2004). Use of the Herves element from A. gambaie to disable the mosquitoes' ability to transmit malaria has also been investigated (Arensburger, Kim et al. 2005). These studies present utilization of transposons, but it is possible to use retrotransposons as vectors as well. Insertion of gene sequences into transposons is done by replacing the transposase gene, whereas inserting gene sequences into retrotransposons involves insertion into the 3’UTR (Largaespada 2003).

Retrotransposons boast a wide variety of uses, and the elements recovered in this work are no exception. Though a significant amount research will need to be done to implement the recovered elements for any of the aforementioned uses, this study lays the groundwork. 


\section{Literature Cited}

Allen, M. L., A. M. Handler, et al. (2004). "piggyBac transformation of the New World screwworm, Cochliomyia hominivorax, produces multiple distinct mutant strains." Med Vet Entomol 18(1): 1-9.

Ames, C. and B. Turner (2003). "Low temperature episodes in development of blowflies: implications for postmortem interval estimation." Med Vet Entomol 17(2): 178-86.

Arensburger, P., Y. J. Kim, et al. (2005). "An active transposable element, Herves, from the African malaria mosquito Anopheles gambiae." Genetics 169(2): 697-708.

Batzer, M. A. and P. L. Deininger (2002). "Alu repeats and human genomic diversity." Nat Rev Genet 3(5): 370-9.

Benecke, M. and J. D. Wells (2001). DNA Techniques for Forensic Entomology. Forensic Entomology: The Utility of Arthropods in Legal Investigations. J. L. C. Jason H. Byrd. Boca Raton, CLC Press LLC.

Biedler, J. and Z. Tu (2003). "Non-LTR retrotransposons in the African malaria mosquito, Anopheles gambiae: unprecedented diversity and evidence of recent activity." Mol Biol Evol 20(11): 1811-25.

Bushman, F. (2002). "Constructing the vertebrate genome: evidence from eels that LINEs mobilize SINEs." Mol Cell 10(5): 961-2.

Byrd, J. H. and J. L. Castner (2001). Insects of Forensic Importance. Forensic Entomology: The Utility of Arthropods in Legal Investigations. J. H. Byrd and J. L. Castner. Boca Raton, CRC press LLC.

Deininger, P. L. and M. A. Batzer (2002). "Mammalian retroelements." Genome Res 12(10): 1455-65.

Dewannieux, M., C. Esnault, et al. (2003). "LINE-mediated retrotransposition of marked Alu sequences." Nat Genet 35(1): 41-8. 
Drew, A. C. and P. J. Brindley (1997). "A retrotransposon of the non-long terminal repeat class from the human blood fluke Schistosoma mansoni. Similarities to the chickenrepeat-1-like elements of vertebrates." Mol Biol Evol 14(6): 602-10.

Edgar, R. C. (2004). "MUSCLE: a multiple sequence alignment method with reduced time and space complexity." BMC Bioinformatics 5: 113.

Edgar, R. C. (2004). "MUSCLE: multiple sequence alignment with high accuracy and high throughput." Nucleic Acids Res 32(5): 1792-7.

Edgar, R. C. (2004). "MUSCLE: multiple sequence alignment with high accuracy and high throughput." Nucleic Acids Research 32(5): 1792-7.

Eickbush, T. H. and H. S. Malik (2002). Origins and Evolution of Retrotransposons. Mobile DNA II. N. L. Craig. Washington D.C., ASM press: 1111-1144.

Furano, A. V., D. D. Duvernell, et al. (2004). "L1 (LINE-1) retrotransposon diversity differs dramatically between mammals and fish." Trends Genet 20(1): 9-14.

Glushkov, S., O. Novikova, et al. (2006). "Divergent non-LTR retrotransposon lineages from the genomes of scorpions (Arachnida: Scorpiones)." Mol Genet Genomics 275(3): 288-96.

Greenberg, B. and D. Singh (1995). "Species identification of calliphorid (Diptera) eggs." J Med Entomol 32(1): 21-6.

Haas, N. B., J. M. Grabowski, et al. (2001). "Subfamilies of CR1 non-LTR retrotransposons have different 5'UTR sequences but are otherwise conserved." Gene 265(1-2): 175-83.

Haas, N. B., J. M. Grabowski, et al. (1997). "Chicken repeat 1 (CR1) elements, which define an ancient family of vertebrate non-LTR retrotransposons, contain two closely spaced open reading frames." Gene 197(1-2): 305-9. 
Harvey, M. L., I. R. Dadour, et al. (2003). "Mitochondrial DNA cytochrome oxidase I gene: potential for distinction between immature stages of some forensically important fly species (Diptera) in western Australia." Forensic Sci Int 131(2-3): 134-9.

Heinrich, J. C., X. Li, et al. (2002). "Germ-line transformation of the Australian sheep blowfly Lucilia cuprina." Insect Mol Biol 11(1): 1-10.

Herke, S. W., J. Xing, et al. (2007). "A SINE-based dichotomous key for primate identification." Gene 390(1-2): 39-51.

Jurka, J., V. V. Kapitonov, et al. (2005). "Repbase Update, a database of eukaryotic repetitive elements." Cytogenet Genome Res 110(1-4): 462-7.

Kaiser, V. B., M. van Tuinen, et al. (2007). "Insertion events of CR1 retrotransposable elements elucidate the phylogenetic branching order in galliform birds." Mol Biol Evol 24(1): 338-47.

Kajikawa, M., K. Ohshima, et al. (1997). "Determination of the entire sequence of turtle CR1: the first open reading frame of the turtle CR1 element encodes a protein with a novel zinc finger motif." Mol Biol Evol 14(12): 1206-17.

Kajikawa, M. and N. Okada (2002). "LINEs mobilize SINEs in the eel through a shared 3' sequence." Cell 111(3): 433-44.

Kapitonov, V. V. and J. Jurka (2003). "The esterase and PHD domains in CR1-like nonLTR retrotransposons." Mol Biol Evol 20(1): 38-46.

Kidwell, M. G. and D. Lisch (1997). "Transposable elements as sources of variation in animals and plants." Proc Natl Acad Sci U S A 94(15): 7704-11.

Kumar, S., K. Tamura, et al. (2004). "MEGA3: Integrated software for Molecular Evolutionary Genetics Analysis and sequence alignment." Brief Bioinform 5(2): 150-63.

Lander, E. S., L. M. Linton, et al. (2001). "Initial sequencing and analysis of the human genome." Nature 409(6822): 860-921. 
Largaespada, D. A. (2003). "Generating and manipulating transgenic animals using transposable elements." Reprod Biol Endocrinol 1: 80.

Luan, D. D., M. H. Korman, et al. (1993). "Reverse transcription of R2Bm RNA is primed by a nick at the chromosomal target site: a mechanism for non-LTR retrotransposition." Cell 72(4): 595-605.

Malik, H. S., W. D. Burke, et al. (1999). "The age and evolution of non-LTR retrotransposable elements." Mol Biol Evol 16(6): 793-805.

Martin, S. L. (2006). "The ORF1 Protein Encoded by LINE-1: Structure and Function During L1 Retrotransposition." J Biomed Biotechnol 2006(1): 45621.

Martin, S. L., J. Li, et al. (2000). "Deletion analysis defines distinct functional domains for protein-protein and nucleic acid interactions in the ORF1 protein of mouse LINE-1." $\underline{\mathrm{J}}$ Mol Biol 304(1): 11-20.

McClintock, B. (1956). "Controlling elements and the gene." Cold Spring Harb Symp Quant Biol 21: 197-216.

McClure, M. A. (1991). "Evolution of retroposons by acquisition or deletion of retrovirus-like genes." Mol Biol Evol 8(6): 835-56.

Mizuuchi, K. (1992). "Transpositional recombination: mechanistic insights from studies of mu and other elements." Annu Rev Biochem 61: 1011-51.

Murata, S., N. Takasaki, et al. (1993). "Determination of the phylogenetic relationships among Pacific salmonids by using short interspersed elements (SINEs) as temporal landmarks of evolution." Proc Natl Acad Sci U S A 90(15): 6995-9.

Nishihara, H., Y. Satta, et al. (2005). "A retroposon analysis of Afrotherian phylogeny." Mol Biol Evol 22(9): 1823-33. 
Novikova, O., E. Sliwinska, et al. (2007). "CR1 clade of non-LTR retrotransposons from Maculinea butterflies (Lepidoptera: Lycaenidae): evidence for recent horizontal transmission." BMC Evol Biol 7: 93.

Otranto, D. and J. R. Stevens (2002). "Molecular approaches to the study of myiasiscausing larvae." Int J Parasitol 32(11): 1345-60.

Posada, D. and K. A. Crandall (1998). "MODELTEST: testing the model of DNA substitution." Bioinformatics 14(9): 817-8.

Ray, D. A., J. Xing, et al. (2005). "Alu insertion loci and platyrrhine primate phylogeny." Mol Phylogenet Evol 35(1): 117-26.

Ren, X., Z. Han, et al. (2006). "Excision and transposition of piggyBac transposable element in tobacco budworm embryos." Arch Insect Biochem Physiol 63(2): 49-56.

Rodriguez, M. A. a. B., W.M. (1983). "Insect Activity and its Relationship to Decay Rates of Human Cadavers in East Tennessee." Journal of Forensic Sciences 28(2): 423432.

Sabrosky, C. W., G. F. Bennett, et al. (1989). Bird blowflies (Protocalliphora) in North America (Diptera: Calliphoridae), with notes on Palearctic species. Washington DC, Smithsonian Institution Press.

Salem, A. H., D. A. Ray, et al. (2005). "Identity by descent and DNA sequence variation of human SINE and LINE elements." Cytogenet Genome Res 108(1-3): 63-72.

Sperling, F. A., G. S. Anderson, et al. (1994). "A DNA-based approach to the identification of insect species used for postmortem interval estimation." J Forensic Sci 39(2): 418-27.

Stevens, J. and R. Wall (2001). "Genetic relationships between blowflies (Calliphoridae) of forensic importance." Forensic Sci Int 120(1-2): 116-23.

Swofford, D. L. (2002). PAUP: Phylogenetic Analysis Using Parsimony (*and Other Methods), Sinauer Associates, Sunderland, Massachusetts). 
Unge, T., S. Knight, et al. (1994). "2.2 A resolution structure of the amino-terminal half of HIV-1 reverse transcriptase (fingers and palm subdomains)." Structure 2(10): 953-61.

van het Schip, F., J. Samallo, et al. (1987). "A new repetitive element of the CR1 family downstream of the chicken vitellogenin gene." Nucleic Acids Res 15(10): 4193-202.

Vandergon, T. L. and M. Reitman (1994). "Evolution of chicken repeat 1 (CR1) elements: evidence for ancient subfamilies and multiple progenitors." Mol Biol Evol 11(6): 886-98.

Wallman, J. F. and S. C. Donnellan (2001). "The utility of mitochondrial DNA sequences for the identification of forensically important blowflies (Diptera: Calliphoridae) in southeastern Australia." Forensic Sci Int 120(1-2): 60-7.

Wells, J. D., T. Pape, et al. (2001). "DNA-based identification and molecular systematics of forensically important Sarcophagidae (Diptera)." J Forensic Sci 46(5): 1098-102.

Wells, J. D. and F. A. Sperling (2001). "DNA-based identification of forensically important Chrysomyinae (Diptera: Calliphoridae)." Forensic Sci Int 120(1-2): 110-5.

Wells, J. D. and J. R. Stevens (2008). "Application of DNA-based methods in forensic entomology." Annu Rev Entomol 53: 103-20.

Wessler, S. R. (2006). "Transposable elements and the evolution of eukaryotic genomes." Proc Natl Acad Sci U S A 103(47): 17600-1.

Whitworth, T. (2006). "Keys to the Genera and Species of blow Flies (Diptera: Calliphoridae) of America North of Mexico." Proceedings of the Entomological Society of Washington 108(3): 689-725

Williams, K. A., F. J. Cronje, et al. (2008). "Identifying flies used for maggot debridement therapy." S Afr Med J 98(3): 196-7.

Xiong, Y. and T. H. Eickbush (1990). "Origin and evolution of retroelements based upon their reverse transcriptase sequences." EMBO J 9(10): 3353-62. 
Supplemental Data 


\begin{tabular}{|c|c|c|c|c|c|c|}
\hline $\operatorname{Vic12r}$ & A TCCCA - A & TGAAA TCA T & A - - GCTATAG & TC TG T G A GCA & C T G GC C GGA T & ATATTTATGG \\
\hline Vic21 & A TCCCA - A & TGAA A TCA T & A - GCTATAG & TC TGTGAGCA & C TGGCCGGAT & ATATTTATGG \\
\hline CVic26 & A TCCCA - A & TGAAA TCA T & A - - GCTATAG & TC TG T G A GCA & C TGGCCGGAT & ATATTTATGG \\
\hline CVic29 & A TCCCA - A & T GAAA TCA T & A - - GCTATAG & TC T G T G A G C A & T TGCCGGA T & ATATTTATGG \\
\hline CVic7 & A TCCCA - A & TGAAA TCA T & - - GCTATAG & TC TGTGAGCA & TGGCCGGA T & T A T T T \\
\hline CVic20 & A TCC C A - A & TGAAA TCA T & - GCTATAG & ГCTGTGAGCA & T GGCCGGA T & ITATTTATGG \\
\hline Cvom15_2 & A TCCCA - A & TGAAA TCA T & С- АС TATAG & TC TG TGAGA A & TGGCCGGA T & A TATTTACGG \\
\hline Cvom1_2 & GA TCCCA - A & TGAAATCA T & C - - ACTATAG & TC TG TGA GCA & TGGCCGGA T & GTATTTACGG \\
\hline CvomCvic.CR1A.Con & А ТССС & TGAA A TCA T & A - GCTATAG & TC TGTGAGCA & T GGCCGGA T & A TATTTATGG \\
\hline Cvom7_2 & ATACCAGCG & TGGTTTTGA & A - A C A G T G C & T C T T C G A - C G & I T A GC TCG - T & GCA T T A GC T A \\
\hline Cvom7 & TA TACCAGCA & TGGTCTTGA & I- ACAGTAT & TC T - & & \\
\hline Cvom6_2 & TA TACCAGCA & TATTCTTGA & - GCAGTGT & C T TCGA-CG & C TAGCTCG - T & C C A T T \\
\hline Cvom2 & T A T A C C A G C A & C T G G TC T T G A & A - - GCA G T G T & TC T T C GA - C G & $\mathbf{C} \mathbf{T} \cdots \mathbf{C} \mathbf{G}-\mathbf{T}$ & CCATTAGCTA \\
\hline CVic27 & TA T A C C A GC - & $\cdots$ TCTTGA & A - GCAATG T & TCTTCGA - T G & TAGCTCG - T & C CATTAGTTA \\
\hline CVic34 & T A T A C C A & C T G A T & - GCAGTGT & TC T TCGA- C G & TAGCTCG - C & C C A T T T - TC TA \\
\hline Cvom8 & TA TACCAGCA & TGGTCTTGA & I - GCAGTAT & TC T TCGA-CG & G C TCG - T & C CATTAGCTA \\
\hline Cvom3_2 & TA TACCAGCA & TGGTCTTGA & I- ACAGTGT & ГC T TCG - - G & TAGCTCG - T & C CATTAGCTA \\
\hline Cvom2_2 & TA TACCAGCA & TGGTCTTGA & A - GCAGTGT & ГC T TCGA - C G & TAGCTCA - T & C CATTAGCTA \\
\hline CVic24 & T A T A C C A & T T GA & A - - GCAGTGT & TC T TCGA - C G & TCG - T & C C A T T A G \\
\hline Vic10 & T A T A C C A GC A & T T GA & I - GCAGTG T & TC T TCGA-CG & TCG - T & C C \\
\hline$c 16$ & TA TACCAGCA & T TG TCT TGA & I - GCAGTGT & TC T TCGA-CG & $\mathbf{T C G}-\mathbf{T}$ & TA \\
\hline Cvic8 & T A T A C C A & TTGTCTTGA & I - GCAGTGC & C T TCGA - C G & TCG $-\mathbf{T}$ & C C A T T T \\
\hline CVic1 & T A T A C C A & T G G T C T T G A & A - - GCA G T G T & TC T T C G A - C G & C T C G - T & C C A T T A GCTA \\
\hline Vic14 & T A T ACCA & T T GA & $\mathbf{A}-\mathbf{G C A}$ & TC T TC & TC G - T & T T A \\
\hline Vic15 & T A T A C C A & T T GA & I - GCAGTGT & TC T TCGA-CG & $\mathbf{G}-\mathbf{T}$ & T TAC \\
\hline Vic31 & TA T A C C A & T T GA & - GCAGTGT & TCTTCGA-TG & $G-T$ & T A \\
\hline CvomCvic & T A T A C C A & T T GA & A - - GCA & TC T T & $\mathbf{G}-\mathbf{T}$ & C C A T T \\
\hline Cvom10_2 & T A C A C C A & G TGGTCTTGA & A - GCAATGT & TC T TCGA - T G & C C C G - T & C C GTTTGCA A \\
\hline CVic22 & T A T A C C A & T T G G & $\mathbf{A}-$ GCA & TC T T C & $\mathbf{G}-\mathbf{T}$ & A A \\
\hline Cvom5_2 & T A T A C C A & T T GA & ITTGTATTGT & T C T T C G A & $\mathbf{C G}-\mathbf{T}$ & I T A G \\
\hline m8_2 & T A T A C C A & T T G A & I - A & TC T T C & C G - C & A A \\
\hline Cvom5 & T A T A C C A & T T T A & A - - GCAGTGT & TC T T C G & C C A - T & $\mathbf{C} \mathbf{C} \mathbf{G} \mathbf{T} \mathbf{T}$ \\
\hline vom4 & T A T A C C A & TGGTCTTGA & A - GCAGTGT & TC T TCGA - C G & C C G - T & C C G T T A GCA A \\
\hline Cvom3 & T A T A & T T G A & $A-G$ & TC T T C & $-T$ & $\mathbf{T} \mathbf{T}$ \\
\hline Cvom11 & T A T A C C A & T T GA & A - GCA & TC T T C G & $\mathbf{G}-\mathbf{T}$ & Г T A \\
\hline Vic11 & A T A C C A & T T GA & $-G$ & TC T TC & $\mathbf{G}-\mathbf{T}$ & A A \\
\hline Vic33 & T A T A C C A GCA & T TGG TC T T GA & A - - GCAGTGT & TC T T C G A & C C C G - T & $\mathbf{C} \mathbf{C} \mathbf{G} \mathbf{T} \mathbf{T}$ \\
\hline vom14_2 & T A T A C C A & T T GA & 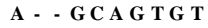 & TC T T C & C C G - T & 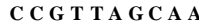 \\
\hline vom16 & $\mathbf{A}$ & T T GA & $--G$ & TC T & $\mathbf{C C G}-\mathbf{T}$ & T T A G \\
\hline Vic32 & A T A C C A & T G G TC T T GA & I- - GCAGTG T & T C T T C T & $\cdots$ & \\
\hline CVic30 & A T A C C A & T T GA & $\ldots$ GCA & T T T C & $\mathbf{T} \mathbf{T}$ & C C \\
\hline CvomCvic.CR1B2.Con & T A T A C C A & C TGGTCTTGA & A - G G A G T G T & TC T T C G & C T A GCC C C & \\
\hline & & TC & & $\mathrm{A}$ & & \\
\hline Vic21 & C A T T & TC TC & A G & T T T T T & A A & TC \\
\hline iic26 & T A C A T T T & TCTC & A GA A & T T T T T & A A & A A \\
\hline Vic29 & TACA T T TAA & T TC TC & G A G & T T T T T C C & C T T T GGAAA & T A T C \\
\hline 7 & A & TC TC & A G & T T T T & A A & TC \\
\hline$c 20$ & A & C TC & G & A & & \\
\hline vom15_2 & CMT T & C C T C & G & T T T T C & A A & A A \\
\hline Cvom1_2 & A C A T T & C C T C & & A A & A A & TC \\
\hline CvomCvic. & T A C A T T T & T T TC & A G A A & T T T T T C & G G A A A & A A A C A A ? \\
\hline Cvom7_2 & C C T T A T & A T A C & & G T & A G & TG \\
\hline vom 7 & & I T A C & A & $\mathbf{G} \mathbf{T}$ & G & TG \\
\hline om6 & C T T T T & [ A T A C & C A & T TTTC & G G A A G & G T T G C T \\
\hline Cvom2 & C C T T T T & Г A T A C & T G C C G GCA & T T TC & G G A A G & G T T GC T A \\
\hline CVic27 & C C T T T T & A T A C & $\mathbf{A}$ & G T & G G A A G & G T T G C T \\
\hline Vic34 & רТT & A T A C & $C T C$ & & G G A A G & GC T \\
\hline vom8 & T T T & T A C & A & $\mathrm{T}$ & G & $\mathrm{BCT}$ \\
\hline vom3_2 & C T T T T & A T A T & T G C C C & A T T T & G A A G & G T T G C T \\
\hline Cvom2_2 & C T T T T & A T A C & C C & G T & G A A A & GC T \\
\hline Vic24 & $\mathbf{G} \mathbf{T} \mathbf{T}$ & I TAC & $\mathbf{A}$ & G T & A G & GC C \\
\hline & G & T A C & & & A G & A T T \\
\hline 16 & G & T A C & A & $T$ & A G & $=\mathrm{C}$ \\
\hline 8 & C T T & T A C & $\mathbf{A}$ & G T & A G & TG \\
\hline CVic1 & C T T T T & A T A A & C A & TTTC & GAA G & G T T GC T \\
\hline Vic14 & I G & I T A C & A & G T & A G & GC T \\
\hline & & & & & & TG \\
\hline Vic31 & G & T A C & A & & & TG \\
\hline CvomCr & C T T & I T A C & $\mathrm{C}$ & G T & A G & GC T \\
\hline Cvom10_2 & C C T T T T & TATATTATAC & T GCC & T T TC & G GA A G & GCTGCTTATG \\
\hline CVic22 & TC T T T T C & G A T A T T A T A C & C G T G C C G G A A & T T TC & G G A A G & G T T T $\cdots \cdots$ \\
\hline 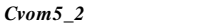 & חד ח & - & - - & & G A A G & GTTGCTAATG \\
\hline$m 8$ & & & T G TC & & & $\mathrm{CT}$ \\
\hline$m 5$ & $\begin{array}{l}0 \\
\end{array}$ & A T A C & C C & TC & A G & TG \\
\hline Cvom 4 & C C T T T T C A G & C TTATTATAC & C G T G C C G G A G & A A T T T C A & T GGAAG & GTTGGTAATG \\
\hline Cvom3 & C T T T T & A T A C & T G & A T & G G A A G & G T T G C T \\
\hline Cvom11 & & T A C & & & & \\
\hline & & A C & & & & TG \\
\hline & I G & $\mathbf{A}-\mathbf{A} \mathbf{C}$ & G & $\mathbf{T}$ & A G & G T T G C T \\
\hline Cvom14_2 & C T T T TCAG & A T A C & T GCC C & T T TC & G GA A G & GTTGCTAATG \\
\hline Cvom16 & A C C T T T T C A G & C A T A T T A T A C & C G T G C C G C T G & A A T T T C C T A T & A T G T T G C A A G & G T T GC T A A T G \\
\hline & & & & & & \\
\hline & C T T T T & & & & G T G T T A G A A G & G T TCC TAA T G \\
\hline$m C v$ & C C T T T T C A G & A TATTATAC & C G T G C C G G A G & T T TC C TA T & A T G T T G G A A G & G T TGCTAATG \\
\hline
\end{tabular}




\begin{tabular}{|c|c|c|c|c|c|c|}
\hline ic12r & T T G T GC T G T T & GC $\ldots \ldots$ & - GCAA A G GC A & ACA A A C C A T T & A G A A A A T C C A & TC T TCA T ACA \\
\hline CVic21 & TTGTGCTGTT & GC $\ldots \ldots$ & - GCAAAGGCA & ACAAACCA T T & AGAAAATCCA & TCTTCA TACA \\
\hline CVic26 & TTGTGCTGTT & GC $\ldots \ldots$ & - GCA A A G GCA & A C A A A C C A T T T & A G A A A A T C C A & TCTTCA T ACA \\
\hline CVic29 & T G T G C T G T T & GC $\ldots \ldots$ & - GCA A A G G C A & ACA A A C C A T T & A G A A A A T C C A & TC T TCA T ACA \\
\hline CVic7 & TGTGCTGTT & GC $\ldots \ldots$ & - GCAAAGGCA & ACA A ACCA T T & AGAAAATCCA & TCTTCATACA \\
\hline CVic20 & TGTGCTGTT & GC $\ldots \ldots$ & - GCAAAGGCA & ACAAACCATT & A GAAAA TCCA & TCTTCATACA \\
\hline Cvom15_2 & TGTGCTGCT & GC $\ldots \ldots$ & - GCAAAGGCA & ACA A ACCA T T & A GAAAA TC C A & TCTTCA TACA \\
\hline Cvom1_2 & TGTGCTGCT & GC $\ldots \ldots$ & - GCAAAGGCA & ACA A A C C A C T & A G A A A A T C C A & TCTTCA TACA \\
\hline CvomCvic.CR1A.Con & TGTGCTGTT & GC $\ldots \ldots$ & - GCAAAGGCA & ACAAACCATT & AGAAAATCCA & ГСТТСАТАС \\
\hline Cvom7_2 & A TCGCCAA T & TC C TA A A A A - & G G G A GA G GC T & A A C A A C C C T G & A A A A T T A TCG & C C C A A T A G C A \\
\hline Cvom 7 & A A CGCCAAT & TCC TAAAAA - & GGGAGAGGCT & A ACAGCCCTG & ATAATTATCG & C C C A A T A GCA \\
\hline Cvom6_2 & A A C G C C A A T & TC C T A A A A A - & G G G A G A G G C T & A A C GACCC T G & A A A A T GATCG & GCCAATAGCA \\
\hline Cvom2 & TA ACGCCAA T & TCC TAAAAA - & GGGAGAGGTT & A A C A ACCC T G & ITTATCG & C C TAATAGCA \\
\hline CVic27 & A ACGCCAAT & TCС TAA A A A - & GGCAGAGGCT & A ACA ACCC TG & A A A ATTATCG & CССАATAGCA \\
\hline CVic34 & A A C G $\ldots$ & $\ldots$ & $\ldots$ & & & $=0$ \\
\hline Cvom8 & ACGCCA A T & TCС TAAAAA - & GGGATAGGCT & A ACA ACC T T & A A A ATTATCG & C C C A A T \\
\hline Cvom3_2 & TAACGCCA A T & TCC TAAAAA - & C GGAGAGGC T & А TCA ACCCTG & T T A T G G & C C C A A T A GCA \\
\hline Cvom2_2 & TAACGCCAA T & TСС TAAAAA - & GC T & C C C T G & Г T A TC G & СССАATAGCA \\
\hline CVic24 & A ACGCCAAT & TCC & A G G C T & C C C T G & T G & C C C \\
\hline CVic10 & TA A C GCCAA T & TCC TA A A A A - & GGGAGA GGC T & ACC C T G & TA T C G & C C C A A T A \\
\hline CVic16 & C C A A T & TCC TAAAAA - & GC T & C C T G & T T A T C G & C C C A A T \\
\hline Cvic8 & TAACGCCA A T & TCC TAAAAA - & GC T & A A TAACCCTG & T T A T C G & C C C A A TAGCA \\
\hline CVic1 & TAACGCCAA T & T C C & S T & C C T G & C G & GCC \\
\hline CVic14 & TAACGCCAA T & A A A - & GGGAGAGGCT & C A C T G & C G & C C T \\
\hline CVic15 & C A C C A A T & TCC TA A A A A - & GGGAGAGGCT & ACCCTG & C G & C C C \\
\hline CVic31 & C C A A T & TCC & $\ldots$ & $\ldots$ & C G & TCC \\
\hline CvomCvic.CR1B1.Con & TAACGCCA A T & TC C T A & GGGAGAGGC T & A ACA ACCCTG & $\Gamma \mathbf{C ~ G}$ & C C C A A T A \\
\hline Cvom10_2 & TA ACGCCA A T & TC & $\mathrm{CT}$ & C C T G & C G & C C \\
\hline CVic22 & $\ldots \ldots$ C CA A T & TCC & GC T & C C T G & C G & C C C \\
\hline$m 5 \_2$ & TAACGCCAA T & TCC & GC T & C C T G & A G & C C C \\
\hline Cvom8_2 & TAA A GCCA A T & TCC & G T & C C T G & T G & C C C \\
\hline Cvom5 & TA A TGCCAA T & TCC & G C C & C C C T T & T A T C G & C C C A A T A \\
\hline Cvom 4 & C C A A T & TCC & C A & C C T G & & $\mathrm{CCC}$ \\
\hline Cvom3 & TAACGCCAA T & A A A - & GGGA & C C T G & TA T C G & C C C \\
\hline Cvom11 & C C A A T & TCC & C A & C C T G & C G & C C C \\
\hline CVic11 & TAACGACAA T & TCCAAA A A A - & S C A & C C C T G & ГTA TC G & C C C A A T \\
\hline CVic33 & TAACGACAA T & TCC & C A & C C T G & Г T A T C G & C C C A A T A \\
\hline Cvom14_2 & A A T & $\mathrm{TC}$ & C A & C T G & $\mathbf{G}$ & $\mathrm{CCC}$ \\
\hline Cvom16 & TAACGCCAA T & A A A A - & GGGAGAGGCA & C C T G & C G & C C C \\
\hline CVic32 & $\ldots \ldots$ & & & C C T G & C G & $\mathrm{CCC}$ \\
\hline CVic30 & TAACGCCA $\mathrm{A}$ & TCC & GC T & C C T G & C G & C C C \\
\hline CvomCvic.C & T A A C GCCAA T & T C C & G C A & C C T G & C G & C C C \\
\hline$c 12 r$ & A T & & G A & G G & & \\
\hline CVic21 & C C - C C T A T & $\mathbf{G} \mathbf{A}$ & G A & T T G G & A T & A T T \\
\hline CVic26 & ACC - C C TA T & T G G A & ACTTGTGGA & T T G G & T T GA T & A T T \\
\hline CVic29 & ACC - C C TA T & G A & G A & T T G G & A T & G A \\
\hline CVic7 & C C - C C T A T & G A & G A & T T G G & $\mathbf{A} \mathbf{T}$ & A $\mathbf{T}$ \\
\hline CVic20 & ACC - C C TA T & G A & G G A & T T G G & A T & A TTAAATAGA \\
\hline Cvom15_2 & C C - GC TA T & G A & G A & C T G G & A T & A TTAAATARA \\
\hline Cvom1_2 & C C - GCTAT & T T G G A & G A & TC T G G & T T G A T & A T TA A A TAGA \\
\hline CvomCvic. & ACC-CCTA T & G A & G A & T T T G G & T T GA T & A T TAAATAGA \\
\hline Cvom7_2 & TC T & & A & $-\ldots \mathrm{G}$ & & A T $\ldots \ldots$ \\
\hline Cvom7 & C T - G T T C T & $\mathbf{E A}$ & G A & $\Gamma--G$ & C C & A $\mathbf{T} \ldots$ \\
\hline Cvom6_ & T T - G T T C T & C A & G G A & T - - G & $\mathrm{CC}$ & A T $\ldots \ldots$ \\
\hline Cvom2 & T T T - G T TCT & GCA C & G G A & $T-\ldots$ & A C T AC C & A T $\ldots \ldots$ \\
\hline CVic27 & TTT-GTTCT & GCACTTTCCA & A A GTTATGGA & $T \ldots G$ & C TAC T & A T $\ldots \ldots$ \\
\hline CVic34 & & & & & & \\
\hline om8 & $C \mathbf{T}-\mathbf{G} \mathbf{T} \mathbf{T} \mathbf{C} \mathbf{T}$ & C A & GA & $\Gamma-\cdots$ & C C & A T $\ldots$ \\
\hline Cvom3_2 & TCT-GTTCT & TC C A & G G A & $\mathbf{T}-\ldots \mathrm{G}$ & A C T A C C & A T $\ldots$ \\
\hline Cvom2_2 & TTT - G TTTT & $\mathbf{C A}$ & G G A & 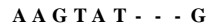 & C TAC C & A T $\ldots$ \\
\hline CVic24 & TTT-GTTCT & & G A & $T \ldots G$ & T C & A T $\ldots \ldots$ \\
\hline CVic10 & T - G T T C T & $\mathbf{A}$ & G A & $\Gamma-\ldots$ & C C & A T $\ldots \ldots$ \\
\hline$c 16$ & $T \mathbf{T}-\mathbf{G} T \mathbf{T} C \mathbf{T}$ & C A & G A & T - - G & C C & A T $\ldots \ldots$ \\
\hline Cvic8 & T T - G T T C T & C C A & G G A & $\mathbf{T} \ldots \mathbf{G}$ & C T A C C & A T $\ldots \ldots$ \\
\hline CVic1 & T T - G T T C T & T A & G G A & $T-\ldots$ & C T A C C & A T $\ldots \ldots$ \\
\hline CVic14 & $T T-G T T C T$ & $\mathbf{C A}$ & G A & $\mathbf{T} \ldots \mathbf{G}$ & C C & A T $\ldots$ \\
\hline & $\mathbf{T}-\mathbf{G} \mathbf{T}$ T C T & & G A & $\ldots G$ & $\mathrm{C} \mathrm{C}$ & $\mathbf{T} \ldots$ \\
\hline 31 & TC T & C A & G A & $-\cdots \mathrm{G}$ & $\mathrm{C}$ & A T $\ldots$ \\
\hline CvomCvic.C & T T T - G T TC T & C A & G G A & $T-\ldots G$ & C C & A T $\ldots \ldots$ \\
\hline Cvom10 & T - G T T C T & C A & A A & $T-\ldots$ & C C & A $\mathbf{T} \ldots$ \\
\hline & C G - G T G T T & C A & G A & $T-\ldots G$ & C C & A T $\ldots$ \\
\hline & $\mathbf{T}-\mathbf{G} A \mathbf{T} \mathbf{C} \mathbf{T}$ & & G A & $-\ldots$ G & $\mathrm{C} \mathrm{C}$ & A T $\ldots \ldots$ \\
\hline Cvom8_ & TC T & C A & & - - - & $-\mathrm{C}$ & A T $\ldots$ \\
\hline Cvom5 & TTT-GTTCT & C A & G G A & $T-\ldots$ & C C A C C & A T $\ldots$ \\
\hline Cvom 4 & ATTTT-GTTCT & GCAC T & A A GTTATGGA & GAGTAT $\cdots$ G & G T T A A C C A C C & A T $\ldots \ldots$ \\
\hline Cvom3 & T T T - G T TC T & TCCA & T G GA & $T-\ldots$ & C C A C C & A T $\ldots$ \\
\hline Cvom11 & T T T - G T T C T & & G G A & & $\mathrm{CC}$ & \\
\hline & $T T-G T T C T$ & & & $\mathbf{T}-\ldots \mathrm{G}$ & C C & A T $\ldots$ \\
\hline & ATTTT-GTTCT & GCA & A A G T & A T $\ldots$ - G & A C C A C C & A T \\
\hline Cvom14_ & ATTTTGTTCT & GCAC T & A A G T & $\mathbf{A} \mathbf{T} \ldots-\mathbf{G}$ & C C A C C & A T $\ldots \ldots$ \\
\hline om16 & G T T C T & C C A & G A & $\Gamma-\mathbf{G}^{-}$ & C C & \\
\hline & T T T T - G C T C T & G C A C & G T & & C C & 81 \\
\hline & C T T T & & & $--G$ & $\mathrm{CC}$ & \\
\hline mCvic.CR1B2.Con & ATTTT-GTTCT & TCC A & AAGTTATGGA & G A G T & A C C A C C & A T $\mathbf{T} \cdots$ \\
\hline
\end{tabular}




\begin{tabular}{|c|c|c|c|c|c|c|}
\hline Vic12r & T T G TGA $\cdots$ & $\cdots$ TGGAAA $\mathbf{T}$ & A A A C GAAAA & GGAGGTT TC T & C ACA TAT - G & C A G T T T G GA T \\
\hline Vic21 & T T G TGA $\cdots$ & $\cdots$ TGGAAA T & A ACGAAAA & GGAGGTTTC T & ACA TAT - G & CAGTTTGGAT \\
\hline CVic26 & T T G TGA $\cdots$ & $\cdots$ TGGAAA T & A A ACGAAAA & GGAGGTTTC T & ACA TAT - G & CAGTTTGGAT \\
\hline CVic29 & T T G TGA - - - & $\cdots$ TGGAAA T & A A ACGAAA A & GGAGGTTTCT & ACA TAT & CAGTTTGGAT \\
\hline CVic7 & T TGTGA $\cdots$ & $\cdots$ TGGAAA T & A A ACGAAA & GGAGGTTTCT & ACA TAT & CAGTTTGGAT \\
\hline CVic20 & T TG TGA - - & $\cdots$ TGGAAA $T$ & A ACGAAAAA & G A G T T T TC T & АС А TA T $\cdots$ G & G T T T \\
\hline Cvom15_2 & T TG TGA $\cdots$ & $\cdots$ TGGAAA T & A A C GGA A A A & GGAGGTTTC T & ACA T A T - G & AGTTTGGAT \\
\hline Cvom1_2 & T TG TGA $\cdots$ & $\cdots$ TGGAAA T & A ACGGAAA & GGAGGTTTCT & ACA TAT - G & AGTTTGGAT \\
\hline CvomCvic.CR1A.Con & TTGTGA & $\cdots$ TGGAAA $T$ & A A ACGAAAA & GGAGGTTTCT & ACA TAT & CAGTTTGGAT \\
\hline Cvom7_2 & T TG TGAAA T & A T $\ldots \ldots$ & $\ldots \ldots$ & $\ldots$ & - GG TACC G G & C AGTATGGC T \\
\hline Cvom $7^{-}$ & T G T G A & T T T A G A G T C & C A A C $\ldots \ldots$ & - - A A T T TA T G & C A GCGACCGG & GC T \\
\hline vom6_2 & T G T G A & G A G T C & A C $\ldots \ldots$ & - - AА T TTACT & A GC G A A C G G & I GTATGGCT \\
\hline Cvom2 & T T G T G A & TT T TGGAGT T & A A C $\ldots \ldots$ & - - АА T TTAA T & AGCGAC C GG & GGC T \\
\hline CVic27 & TTGTGAAA T & T T TAGAG TC & C AAC $\ldots \ldots$ & - - AА T T TACT & C A GCGACCGG & CAGTATGGCT \\
\hline CVic34 & $\ldots \ldots$ & $\ldots \ldots$ & $\ldots \ldots$ & $\ldots \ldots$ & $\ldots \ldots G$ & A GTATGGCT \\
\hline Cvom8 & $\mathbf{T}-\ldots$ GA & T T T A GA G T C & C AAC $\ldots \ldots$ & - - A A T T T A C T & $\ldots \ldots$ & GA A T G GC T \\
\hline vom3_2 & T G T G A & $\mathbf{T} \mathbf{T} \mathbf{T}$ & AC $\ldots \ldots$ & - - АA TA TACT & CAGTGACCGA & GC T \\
\hline vom2_2 & T T G T G A & $\mathbf{T} \mathbf{T} \mathbf{T}$ & A C $\ldots \ldots$ & T T T A C T & TAGCGAC C GG & A G T A T \\
\hline CVic24 & T T G T G A & T T T A & C AAC $\ldots \ldots$ & - - АА T T T TCT & C A GCGAC C GG & C A G T A T \\
\hline Vic10 & T T G T G A & T T T & С A A C $\ldots \ldots$ & T T T AC T & G C G A C C G G & T A T \\
\hline Vic16 & G T G & T T T & C - - - - & T T T A C T & G C G A C & A T \\
\hline$c 8$ & G T & TC & C $\ldots \ldots$ & $--A$ & G & \\
\hline$c 1$ & T G TG & $\mathbf{T} \mathbf{T} \mathbf{T}$ & AC $\ldots \ldots$ & T T T AC T & G C G A C & C T \\
\hline Vic14 & T G T G & T T T & A C $\ldots \ldots$ & T T T A C T & G C G A C C G G & G T A T \\
\hline Vic15 & T G T G & G T C & A C $\ldots \ldots$ & T T T A C T & G C G A C C G G & A C T \\
\hline CVic31 & Г G T G & G T C & A C $\ldots \ldots$ & T T TAC T & G C G A C & \\
\hline$m C v i$ & $\mathrm{~T}$ & G TC & C - . . - - & $--A$ & G A C & C T \\
\hline vom10_2 & G T & G TC & C - . . - - & $-A$ & G & C A \\
\hline Vic22 & T G T G & TC T & A C $\ldots \ldots$ & T T A A C T & G C G A C C G G & T AC \\
\hline vom5_2 & G T & TC T & C - . - . - & T T T AC T & G A C & TAC \\
\hline vom8_2 & Г G T & G TC & A C $\ldots \ldots$ & T T T T C T & G C G A C C G G & C A G T A C \\
\hline $\mathrm{m} 5$ & G T & I TC & C $\ldots \ldots$ & T T TACT & $\ldots$ & $\ldots$ \\
\hline om 4 & T G T G & TC T & C $\ldots \ldots$ & $--A$ & G A C C G G & CAGTATGGCT \\
\hline vom3 & T G T G & TC & C - - - - - & - - A & G C G G C C G G & TAC \\
\hline om11 & G T & T C & C - . - - & ГT T TACT & G A C & TAC \\
\hline Vic11 & G T G & TC T & A C $\ldots \ldots$ & T T T A C T & GC G A C & TAC \\
\hline c33 & G T & TC T & A C $\ldots \ldots$ & T T T A C T & G A C & C T \\
\hline om14_2 & G T & TC & C - . . - & T T T A C T & G G & C T \\
\hline om16 & $\mathbf{A} \mathbf{T}$ & G TC & AC $\ldots \ldots$ & $--A$ & G A C C G G & TACGGC T \\
\hline 32 & $\mathbf{T}$ & TC & $c \ldots$ & $--A$ & G G & TAC \\
\hline Vic30 & G T G & G T T & A C $\ldots \ldots$ & T T T A C T & GCGAC - - & $\ldots \ldots$ - G GC T \\
\hline$o m C v$ & $\mathrm{~T}$ & G T C & AC $\ldots \ldots$ & & & \\
\hline$c 12 r$ & & & G.... & & $\Gamma \mathbf{A}$ & \\
\hline Vic21 & G & C T & G - . - - & A T C C G & I A & A A \\
\hline Vic26 & $T G$ & C T & A G G - - - - & A T C C G & G T G G T A & A C 1 \\
\hline$c 29$ & G TG & C T & GG - . . - & C A T C C G & G T G G T A & A C 7 \\
\hline Vic7 & S G T G A A & T A A A & Г A GG - - - - & C A T C C G & GA GGTGGTA & A T AC T \\
\hline Vic20 & & C T & G - . - - & C G & G T A & A A \\
\hline$m 15$ & G & $\mathrm{C} \mathbf{T}$ & - . . - & $\mathbf{G}$ & G T G & A \\
\hline$m 1 \_2$ & TO & C T & G - - - & A T C C G & A G G T G G T A & AC 1 \\
\hline$m C v i$ & G T G & ACT & G $\ldots$ & C A T C C G & TGAGGTGGTA & A A T A C \\
\hline vom7_2 & C G T & C G & A C T T G C & T T C C - & $\ldots \ldots$ & - - TA T $\mathbf{T}$ \\
\hline vom 7 & G G & C G & I T GC & C - & $\cdots \cdots$ & - - TA T C \\
\hline$m 6$ & & C G & C & & $\ldots$ & - - \\
\hline om2 & G T & C G & T T G C & T TCC - & $\ldots \ldots$ & - - TA T T \\
\hline & G T & I C G & A C T T G A & T T TC C - & $\ldots$ & - $\mathbf{T A} \mathbf{T} \mathbf{C}$ \\
\hline Vic34 & C A C & C T A C G & GGATACTTGC & TAGCCTTCC - & $\ldots \ldots$ & - TA TCGGAA \\
\hline om8 & & $\cdots$ & $\cdots \cdots$ & $\ldots-\cdots$ & $\cdots \cdots$ & G T A \\
\hline ? & G & IC G & ACT T T C & C T T & $\ldots$ & - \\
\hline om2_2 & G & C G & T GC & $T-$ & $\cdots$ & A T C \\
\hline$c 24$ & G G & C G & T T G C & C T T T C - & $\ldots \ldots$ & - - \\
\hline Vic10 & G T & A C G & ICCTGC & C T T C C - & $\ldots \ldots$ & - - TA T TC \\
\hline ic16 & G G & C G & AC C T GC & C C - & $\ldots \ldots$ & - - TA T TC \\
\hline 8 & & & & & $\ldots$ & $\ldots$ \\
\hline & & C G & & & $\ldots$ & - \\
\hline & G T & C G & T T G C & C T TCC - & $\ldots$ & A T C \\
\hline Vic15 & TCG TAGAGG & ACGCTCTACG & GGAGACT TGC & TAGACT TCC - & $\ldots \ldots$ & - - TA TCGGAA \\
\hline Vic31 & $\ldots \ldots$ & $\ldots \ldots$ & $\ldots \ldots$ & $\ldots$ & $\ldots \ldots$ & $\ldots$ \\
\hline vomCv & G G & & & & $\ldots \ldots$ & \\
\hline n10 & & T G & $\mathrm{C}$ & C - & $\cdots$ & TC \\
\hline & G & C G & C & C T T C C - & $\ldots \ldots$ & --7 \\
\hline om5_2 & G T A & A G G & C T T G C & C T T A C - & $\ldots$ & - - TA T T \\
\hline Cvom8_2 & TCGTAGAGG & ACGCTCTACG & C T T GC & C T T C C - & $\ldots \ldots$ & -- \\
\hline & - & - & A C T T G C & & & A A \\
\hline & G T A & C G & T T GC & C T T & $\cdots-$ & A T C \\
\hline & G T A & C GC TC T & C T T G C & C T TC C - & - & T T C \\
\hline Cvom11 & C G T A G & C C GCTCTACG & C T T GC & T TGCCTTCC - & $\ldots$ & - - T A T T C \\
\hline Vic11 & G T A & C GCTCTACG & GGGGACT TGC & C T T C C - & $\ldots$ & - - TA T T \\
\hline & G T A & GC TC T AC G & C T T G C & T T GC C T T C C - & & - T A T C \\
\hline & G & C G & & G - & & A A \\
\hline & G T A & C G & & C T T C C - & & A T C \\
\hline c32 & - & - - GC TCTACG & GGGGACT T GC & T T GCC T TCC - & $\ldots \ldots$ & - TA TCGGAA \\
\hline CVic30 & T T C G T A G & ICGCTCTACG & GGGGACT TGC & T T GCC T & $\ldots \ldots$ & - - TA T C \\
\hline$n C$ & G A G G & C T A C G & G G G G A C T T G C & T T G C C T & $-\cdots$ & G A A \\
\hline
\end{tabular}




\begin{tabular}{|c|c|c|c|c|c|c|}
\hline$c 12 r$ & A GCT T T & C T T G & CGATTTTGCT & C C A $\cdots \cdots$ & TCGTCACCC T & G G A C A T C \\
\hline & GGAAGC T T T & TGAGCACT TG & CGATTTTGCT & C C A $\cdots$ & TCG TCACCC T & GGACATCAAG \\
\hline CVic26 & GGAAGC T T T & T GA GCACT T G & C GATTTTGCT & C C A $\cdots \cdots$ & TCGTCACC C T & GGACA TCA A G \\
\hline CVic29 & GGAAGCTTT & TGAGCACTTG & CGATTTTGCT & C C A $\ldots$ & TCGTCACCCT & GGACATCAAG \\
\hline CVic7 & GGAAGC T T T & GAGCACT TG & GATTTTGCT & C C A $\ldots$ & C C G TCACCCT & GGACA TCA A G \\
\hline CVic20 & GGAAGCTT T & GAGCACT TG & GATTTTGCT & C A $\cdots$ & C G TCACCC T & GGACATCAAG \\
\hline Cvom15_2 & GGAAGCTTT & TGAACACA T G & GATTTTGTT & C C A $\ldots$ & TCG TCACCC T & GGACATCAAG \\
\hline Cvom1_2 & GGAAGC T T T & TGAACACA T G & CGATTTTGTT & C C A $\cdots \cdots$ & TCG TCACCC T & GGACATCAAG \\
\hline CvomCvic.CR1A.Con & GGAAGCTTT & TGAGCACT TG & CGATTTTGCT & C C A $\cdots \cdots$ & TCG TCACCC T & GGACATCAAG \\
\hline Cvom7_2 & AATGGTATC & T T TCCATACA & C G T T T T GGC & GAAAG TAA A G & GGTGGCTCT & IGATATTTCT \\
\hline Cvom7 & AATTATGTC & T TCCATACA & C G T T T T GGC & A A A A GCA A A G & GGTGGCTCT & AGATATTTCT \\
\hline Cvom6_2 & $\cdots$ - & T T TCCATACA & C GTTTTGGC & GAAAGTAA & G T T GGC TC T & AGATATTTCT \\
\hline Cvom2 & A A A T G G T G T C & G T T C C A C A C A & C TG TA T T G G C & GAA A G T A A A G & G G T G GC TC T & A GATATTTCT \\
\hline CVic27 & A A T G G T $\cdots$ & G T TCTATACA & C C G T T T T G G C & A T A A A G & G G T A T C TC T & T A T T T C T \\
\hline CVic34 & AATGGTGTC & GTTCCATACA & C G T T T T G G C & G T A A A G & G G T GGC TC T & T T TC T \\
\hline Cvom8 & AATGGTGTC & GTACCATATA & CGTTTTGGC & G T A A A G & GGTGGCTCT & IGATATTTCT \\
\hline Cvom3_2 & A A T G G T GC C & A TTCCATACA & C G T T T T GGC & G T A A A G & AC TGGCTCT & AGATATTTCT \\
\hline Cvom2_2 & A A T G G T G T C & G T TCCA T A C A & C C G T T T T C G C & GAAA G TAA A G & T GGAGGCTC T & AGATATTTCT \\
\hline CVic24 & G T G TC & TA T AC A & C G TTTTGGC & G & C A C T & C T \\
\hline CVic10 & A T G G T GCC & GTTCCATACA & C G TTTTGGC & GA A & C T C T & T T T TC T \\
\hline & A T G G TGCC & GTTCCATACA & CGTTTTGGC & G & C T C T & C T \\
\hline 88 & T G T C & T A C A & C CGTTTTGGC & G & C T C T & C T \\
\hline$c 1$ & G T G T C & G T T C C A T A C A & C C G T T T T G G C & G A A & G G T G G C T C T & A GA T A T T T C T \\
\hline$c 14$ & TG TC & T A C A & C G TTTTGGC & G & C TC T & A T \\
\hline ic15 & A A TGGTGTC & C A T A C A & C G T T T T GGC & G & C TC T & C T \\
\hline$c 31$ & G T G T C & T A C G & C GTTTTGGC & & C TC T & C T \\
\hline CvomCvic & A A A TGGTGTC & T A C A & C C G T T T & G & C T C T & C T \\
\hline Cvom10_2 & A G T G G T GC C & GTTCTATTCA & C CGTTTTGGC & & GC TC C & TC T T TC T \\
\hline CVic22 & T G T C & T T C A & C C G T T T T G GC & & C T & \\
\hline Cvom5_2 & A T G G T G C C & G T T C C & C G T T T T G GC & GA A & C T C T & C T \\
\hline m8_2 & G T G C C & T TCA & TGTTTTGGC & & C T C T & C T \\
\hline Cvom5 & G T C C C & G T TCCATTCA & C CGTTTTGGC & GA A & C T C T & $\mathrm{CT}$ \\
\hline Cvom 4 & A T G G T G C C & G T TCCATTCA & CCGTTTTGGC & G & C TC T & T A T T T C T \\
\hline Cvom3 & T G C C & T C C A & C CGTTTTGGC & & C T & \\
\hline vom11 & G T G C C & TC C A & C G T T T T G GC & G & C T C T & C T \\
\hline CVic11 & G T G C C & T T C A & C GTTTTGGC & G & C T C T & C T \\
\hline CVic33 & A G T G G T G C C & G T TCCATTCA & C CGTTTTGGC & GA A & C T C T & T A T T T C T \\
\hline Cvom14_2 & G T G C C & T T C A & C CGTTTTGGC & & C T C T & \\
\hline Cvom16 & GCC & C C A & G T T T & & C T & \\
\hline Vic32 & G T G T C & G T T C C & C C G T T T T G G C & GA A & C T C T & A T C T \\
\hline Vic30 & G T G TC & T T C A & CGTTTTGGC & A G & $\mathrm{CC}$ & C T \\
\hline CvomCvi & A A T G G T GC C & G T TC C A T TCA & C G T T T TGGC & G A A & C T C T & \\
\hline & C A & A & Г A & & G A & \\
\hline Vic21 & T TCA & C TCT & T TGGA A T G T A & A & G A G A & TC \\
\hline CVic26 & I T TCA & C TCT & TGGAATGTA & A & A G A & TC \\
\hline CVic29 & C G C A T T C A & ACTCTGCAAA & C TGGAATGTA & A A & G A G A & A A T T T C \\
\hline CVic7 & A T T C A & C TC TGCAAA & T TGGAATGTA & & A G A & A T T T C \\
\hline c20 & TCA & A A A & TA & & G A & \\
\hline m15 & T T C A & C TCTGCTAA & G T A & & G A & TC \\
\hline Cvom1_2 & T T C A & C TCTGCTAA & T G T A & A & G A G A & C T T C \\
\hline CvomCvic. & C G C A T T C A & AC TC TGCAAA & C T G G A A T G T A & A A & A A T G C G A G A & A A A T T TC \\
\hline Cvom7_2 & G T T T G & - - - - - & - CAGAATATG & & $--\mathbf{A}$ & C T T A \\
\hline Cvom7 & T T G & $\ldots$ & T G & & $-\cdots \mathbf{A}$ & T A \\
\hline$m 6$ & T T T G & $\cdots \cdots$ & - TAGAGTA T A & C G & T T T $\cdots$ - A & GCA A A A C T T A \\
\hline Cvom2 & G G G T T T G & $1 \ldots \ldots$ & - TAGAGTATG & G T A & Г T T T $\cdots$ & $\ldots \ldots$ \\
\hline CVic27 & T T T G & $\ldots \ldots$ & - CAGAGTA T G & C G & T T T $\cdots \mathbf{A}$ & A A C T T A \\
\hline CVic34 & G T T T G & $\ldots$ & - TAGAGTATG & & T T T $\ldots$ A & C T T A \\
\hline Cvom8 & T T T G & $\cdots-$ & T G & G & $\cdots \mathbf{A}$ & T A \\
\hline m3_ & T T T G & $\ldots$ & - TAGAGTATG & C G & $\mathbf{T} \mathbf{T} \mathbf{T} \cdots \mathbf{A}$ & C T T A \\
\hline Cvom2_2 & GC G T T T G & A $\ldots \ldots$ & - TAGAGTATG & C G & $\mathbf{T} \mathbf{T} \mathbf{T}-\cdots \mathbf{A}$ & A ACT T A \\
\hline CVic24 & T T T G & A $\ldots \ldots$ & $-\mathbf{T} A$ & & T T T $\cdots$ A & T A \\
\hline & T T T G & $\ldots$ & T G & & T T T $\ldots$ - $\mathbf{A}$ & C T T A \\
\hline 16 & T T G & $\cdots \cdots$ & T G & & $\cdots \mathbf{A}$ & \\
\hline 8 & T T G & $\ldots \ldots$ & - TAGAGTA T G & G & T T T $\cdots$ - A & T A \\
\hline CV & T T TG & A $\cdots \cdots$ & - TAGAGTATG & GC A & T T T $\cdots$ - & A A C T T A \\
\hline ic14 & T T T G & A $\cdots \cdots$ & $-T$ & & $\Gamma \mathbf{T} \mathbf{T} \cdots \mathbf{A}$ & T A \\
\hline & G T T T G & $\ldots$ & & & T T T $\cdots$ - A & ACT TA \\
\hline 31 & T T T G & $\ldots \ldots$ & $-\mathrm{T}$ & & $-\cdots \mathbf{A}$ & \\
\hline$m C$ & T T T G & $\ldots \ldots$ & T A T G & C G & T TTT & T A \\
\hline Cvom10_2 & G G G T TCG & A $\cdots \cdots$ & - TAAGGTGTG & A $\ldots$ & $\ldots \ldots$ & A A C T T A \\
\hline CVic22 & G G C G T T C G & A $\cdots \cdots$ & - T A G G G T G T G & GCA & C TC T T $\cdots$ A & AC T TA \\
\hline & G C G T T C G & $\ldots$ & - TAGGGTGTG & & $\Gamma$ C G T $\cdots$ - A & T T T A \\
\hline$m 8$ & G T T C G & & & & A & \\
\hline$n 5$ & G T T C G & $-\cdots$ & - A AGGGTGTG & G & $\cdots \mathbf{A}$ & T A \\
\hline Cvom 4 & G G C G T C C G & A $\cdots \cdots$ & - TAGGGTG T G & GC A & C TCT T $\cdots$ A & A A C T T A \\
\hline Cvom 3 & G T T C G & A $\cdots \cdots$ & - TAGGGTG T G & GC A & Г С T T $\cdots$ A & T A \\
\hline vom11 & T T G & $\ldots-\cdots$ & & & $--\mathbf{A}$ & \\
\hline & C G G & & & & & \\
\hline & T T C G & A & - TAGGGTGTG & & $-\cdots \mathbf{A}$ & CA \\
\hline Cvom14_2 & G C G T T C G & A $\ldots \ldots$ & - TAGGGTGTG & GCA & C TCT T $\cdots$ A & A ACT TA \\
\hline Cvom16 & G T T C G & A $\ldots \ldots$ & - TAGGGTGTG & G TA T G G T GCG & C TCTT & TCGAAAC T TA \\
\hline & T TC G & $\cdots$ & - TAGGGTGTG & GCA TGGTGCG & C TCT T $\cdots$ A & TCGAAACT TA \\
\hline & G G C G T T C G & - - - & $-\cdots$ & & 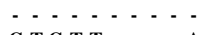 & \\
\hline$m C$ & A GGCGTTCG & $\ldots$ & G G G T G T G & GCATGGTGCG & $--\mathbf{A}$ & TCGAAAC T T \\
\hline
\end{tabular}




\begin{tabular}{|c|c|c|c|c|c|c|}
\hline CVic12r & TCA GAA T A T T T & T GG TAAAA A T & GG $\cdots \cdots$ T T & GAT $\ldots \ldots$ & - AATTATCTG G & G A A G A T A \\
\hline CVic21 & T C A G A A T A T T & T G G T A A A A A T T & GG - . - T T & GA T $\cdots \cdots$ & - A A T TA TC T G G & GA A G A T A G A A \\
\hline CVic26 & C A GAA TA T T & GG TAAAAA T & GG $\cdots \cdots$ T T & A T $\ldots \ldots$ & - AATTATCTG G & GAA G A T A G A A \\
\hline CVic29 & C A GAA T A T T & G G TAAAAA T & GG $\cdots \cdots$ T T & $\mathbf{A} \mathbf{T} \ldots \ldots$ & - AATTATCTG G & GAA G A T A G A A \\
\hline CVic7 & TCA GA A T A T T & G G T A A A A A T & GG $\cdots \cdots$ T T & GA T $\cdots \cdots$ & - АA T TA TCTG G & GA A G A $\mathbf{T}$ \\
\hline CVic20 & TCAGA A T A T T $T$ & [GG TAAAAA T & GG $\cdots \cdots$ T T & GAT $\ldots \ldots$ & - AATTATCTG G & GAAGATAGA A \\
\hline Cvom15_2 & T C A G G A T A T T & G G T G A G A A T & GG $\cdots \cdots$ T T & G A T $\cdots \cdots$ & - A A T TACC TG G & G A A G A T A G A A \\
\hline Cvom1_2 & TCAGGATA T T & GG TGAGAA T & GG $\ldots \ldots$ TG & GA T $\ldots \ldots$ & - AATTACC TG G & GAAGATAGA A \\
\hline CvomCvic.CR1A.Con & TCA GAA T A T T $T$ & G G TAAAAA T & GG $\cdots \cdots$ T T & GAT $\ldots \ldots$ & - AATTATCTG G & GAA G A T A G A A \\
\hline Cvom7_2 & T T GA T T T T G G & [ G T C G G T A T C & AACTTCTATC & GATTTATATC & GAGCTTTCTC A & A GAAATCGCA \\
\hline Cvom7 & T T GC T T T T G G & G TCGATAA T & A ACT TCTCTC & GATTTATATC & GAGCTTTCTC A & A G A T C \\
\hline Cvom6_2 & T T GC T T T T G G & G TCGATAA T & A ACT TCTCTA & GACTTATATC & GAGCTTTCTC A & A GAGATCGCA \\
\hline Cvom2 & $\ldots-\ldots$ G G & G TCGGTAAT & A ACTTCTCTC & GATTTATATC & GAGCTTTCTC A & A G A G A T C \\
\hline CVic27 & T T G C T T T T G G & T G T C T A T A A T & A ACT TCTCTC & GA T T TATATC & GAGCTTTCTC A & A G A G A T C G C A \\
\hline CVic34 & T T GC T T T T G G & T G TCGGTAA T & A GCTTCTCTC & GATTTATA TC & GATCTTT $\ldots$ & $\cdots$ ACC \\
\hline Cvom8 & T T T G G & G T C G & A A $\cdots \cdots$ & $\cdots \cdots C$ & GAGC TTTC TC A & A G A G A T \\
\hline Cvom3_2 & T T GC T T T T G G & G TC GA TAA T & A ACT TC TCTC & GATTTATATC & GAGCTTTCTC A & A G A G A T \\
\hline Cvom2_2 & T G T T G G & TAA $\mathbf{T}$ & A A TTTCTCTC & ATTTATATC & GAGCTTTCTC A & A GATC \\
\hline CVic24 & T T GC T T T T G G & G T C G G TAA T & AATTTCTCTC & GATTTATATC & GAACTTTCTC A & A G A T \\
\hline CVic10 & T T T T G G & T G T C G & ГTC TCTC & A TC C & GTTTTCTC A & C A \\
\hline CVic16 & T T T G G & A A $\mathbf{T}$ & TC & TC C & TC TC A & C A \\
\hline Cvic8 & T T T G G & A A T & T T TC TC TC & TA TC & GAGTTTTCTC A & C A \\
\hline CVic1 & I T T T G G & A A T & T TC TC TC & TA TA TC & GCTTTCTC A & T A \\
\hline CVic14 & C T T T T G G & T G T T G & A ACTTCGTTC & GA T T TATATC & GAGCTTTCTC A & GA T \\
\hline CVic15 & $\Gamma \mathbf{T}$ & A T & TC & TC & TCTC A & C A \\
\hline CVic31 & G G & A T & TC & TC & ГC TC A & C A \\
\hline Cvom Cvic & G G & A T T & TC & A T C & GCTTTCTC A & C A \\
\hline Cvom10_2 & ГT T T T G & A A T & T TC TC TC & TA T A T C & G A G C T T T C T T A & C A \\
\hline CVic22 & {$[\mathbf{T} T$} & $\triangle \mathrm{AC}$ & C TC & TC & GCTTTTTC A & A T \\
\hline Cvom5_2 & T T T G G & A T & TC & TC & G G T T TC TC A & $\mathbf{C} \mathbf{A}$ \\
\hline Cvom8_2 & T T T G A & A T & TC & A T C & C T T TC TC A & C A \\
\hline Cvom5 & $\begin{array}{r}\mathbf{T} T \\
\mathbf{T}\end{array}$ & $\mathbf{A} \mathbf{T}$ & TC & TC C & TC TC A & $\mathbf{C A}$ \\
\hline Cvom 4 & T T T T G G & A A T & C T TCC T TC & GATTTATATC & GAGCTTTCTC A & GA T \\
\hline Cvom3 & G G & A T & TC & TC & TC TC A & C A \\
\hline Cvom11 & T T T G G & A A T & С TC TC & A T C & GAGCT T TC T C A & C A \\
\hline CVic11 & G G & A A T & TC & A T C & GCTTTCTC A & C A \\
\hline CVic33 & T T T T G G & T G T C G G T A A T & G AC T TC TC TC & GA T T TATATC & GAGCTTTCTC A & A G A G A T C G C A \\
\hline Cvom14_2 & C T T T T G G & $\ldots \ldots$ & $\ldots \ldots$ & $\ldots \ldots$ & $\ldots \ldots$ & $\ldots \ldots$ \\
\hline Cvom16 & I T T T G G & G T C G & ГС ТС TС & T A T C & GAGCT T TC TC A & A G A G A T C G C A \\
\hline CVic32 & I T T T G G & TCGG & TC & TC & GCCTTCTCA & C A \\
\hline CVic30 & $\cdots$ & A C & TC & A TC & GCTTTCTC A & C A \\
\hline CvomCvi & T T GC T T T T G G & T G T C G G T A A T & G AC T TC TC TC & GATT TATATC & GA GCT T TC T C A & A G A G A T \\
\hline & & & & & & \\
\hline CVic21 & T A & C T G & A A $\mathbf{T}$ & $\cdots \mathbf{A A}$ & C G A & C T \\
\hline CVic26 & T GA & TGA - - TAC T G & A A T & $\therefore-\cdots \mathbf{A}$ & AGC T & C T \\
\hline CVic29 & T C C T T G A A T A & T GA - - T A C T G & A A G A T G G A A T & $\cdots \cdots$ A A A A & G C T A C G A A G & T G A C A G C T \\
\hline CVic7 & T A & A - T AC T G & A A $\mathbf{T}$ & $\cdots \cdots \mathbf{A}$ & C G A A G & \\
\hline CVic20 & T A & C T G & A A $\mathbf{T}$ & $\therefore \ldots$ A A A A & C G A A G & C T \\
\hline Cvom15 & T A & C T G & $\mathbf{A} \mathbf{A} \mathbf{T}$ & $\cdots \cdots$ A A A A & C G A A G & C T \\
\hline Cvom1_2 & T A & A - T A C T G & A A T & $\cdots \cdots$ AAAA & A GC T A C GA A G & $\mathrm{CT}$ \\
\hline CvomCvic. & T GAA T A & A - - T A C T G & A A T & 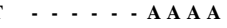 & C GA A G & \\
\hline Cvo & $\cdots$ & T T A & G A T & C & C A A G A & \\
\hline Cvom7 & $-\cdots-1$ & T T A & A A T & $\mathrm{T} \mathrm{T}$ & C A A G A & $\mathbf{A}$ \\
\hline Cvom6_2 & $\cdots$ A T A & T T A & G A A T T & C T C & A G T TCA A GA & C A \\
\hline Cvom2 & C A $\cdots$ G T A & G T T A & GA A T & C TCA TCA A A C & GA G T TC A A G A & TCA A \\
\hline CVic27 & $\cdots \mathbf{A} \mathbf{T} \mathbf{A}$ & G T T A & T G G A A T T & T TCA TCAAA C & A G T A C A A G A & ГC A \\
\hline$C V$ & $\cdots$ & T T A & A A T & & A A T A & A \\
\hline Cvom8 & $\cdots$ A T A & $\Gamma \mathbf{T} \mathbf{A}$ & A T & C & A A T A & \\
\hline Cvom3_ & $\cdots$ - $\mathbf{A}$ T A & T T A & A A T $\mathbf{T}$ & A A C & A G T TCA A G A & C A \\
\hline Cvom2_2 & $\cdots$ - A T A & T T A & A A T & AC & A G T TC A A G A & C A \\
\hline CVic24 & Г - . A T A & G T T A & & & A G T T C A A G A & \\
\hline & $\cdots$ & Г T A & & & A A G A & \\
\hline & $\cdots$ & T A & & & A A & \\
\hline & $\cdots-1$ & T T A & A A T & A A C & A G T T C A A G A & $\mathbf{C A}$ \\
\hline CVic1 & Г - - A T G & T T A & A A $\mathbf{T}$ & A G C & A G T T C A A G A & C A \\
\hline CVic14 & $\cdots$ & T T A & & & A G T T C A A G A & \\
\hline & $\cdots$ & Г T A & & & A G A & \\
\hline & $\cdots$ & T A & & & G T & \\
\hline CvomCv & 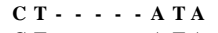 & T T A & $\mathbf{A} \mathbf{T}$ & $\mathrm{C}$ & C A A G A & $\mathbf{A}$ \\
\hline Cvom10 & $\cdots$ - A T A & T T G & G A T & A A T & GAG T TCAAA A & C A \\
\hline CVic22 & $\cdots$ & Г T G & G A T & TCA A A T & A G A & C A \\
\hline & $\cdots$ & T T G & & & A A A G A & \\
\hline Cvom8_ & $\cdots$ & T G & & & G G T & \\
\hline Cvom5 & $\cdots$ & T T G & A T & $T$ & A A A & \\
\hline Cvom 4 & C T $\cdots$ A T A & G T T G & G G A T & C A A A T & G A G T T C A A G A & C A \\
\hline Cvom3 & $\cdots$ & T T G & TGGGA T & TCAAA $T$ & A G T TCA A GA & C A \\
\hline 11 & & T G & & & & \\
\hline & & T G & & & & \\
\hline $\mathrm{CVi}$ & C T $\cdots \cdots$ & T T G & A $\mathbf{T}$ & A $\mathrm{T}$ & C A A G A & $\mathbf{C A}$ \\
\hline Cvom14_2 & $\cdots \cdots$ & $\cdots \cdots$ T G T T G & T A G A T G G G A T & С ТС А ТС АA A T & A A G T T C A A G A & T T A A T T C A \\
\hline Cvom16 & C T $\ldots$ & T G T T G & TA G A T & A A A T $\mathbf{T}$ & GAGT TCAAGA & T TAA T TCA \\
\hline & & T T G & & & & \\
\hline & & T G & & & & \\
\hline mCvic. & C T $\cdots$ & A G T T G T T G & T A G A T G G GA T & & G A G T T C A A G A & \\
\hline
\end{tabular}

Supplemental Figure 1 Sequences obtained from C.vom/C.vic clones alongside consensus clusters 


\begin{tabular}{|c|c|c|c|c|c|c|}
\hline Lser6_2 & CA TA TCCA T G & C TGA T GC T GA & A ACACCTTGG & C G T A C G G GA A & G T C G A G T A C T & TGACAA T G G T \\
\hline Lser7 & САTATCCATG & C TGATGCTGA & A ACACCTTGG & C GTACGGGAA & GTCGAGTACT & TGACA A TGGT \\
\hline Lser5 & CA TA TCCA T G & C TGA T GC T G A & A A C ACCTTGG & C G T A C G G G A A & G T C G A G T A C T & TGACAA T G G T \\
\hline Lser.CR1A1.Con & C A TA TCCA T G & C TGA T GC T GA & A ACACCTTGG & C G T A C G G GA A & G T C G A G T A C T & TGACAA T G G T \\
\hline Lser7_2 & CATATCCATG & C A GA TGCTGA & GAC TCCTTGG & C GA A C G T GGA & G TCGAGTACT & TGACAATGGT \\
\hline Lser10_2 & САТАTССАTG & C TGATGCTGA & A ACACCTTGG & C GA A C G G G GA & GTCGAGTACT & TGACA A TGGT \\
\hline Lser3_2 & САТАTСCATG & C TGATGCTGA & A ACACCTTGG & C GA A C G G G GA & GTCGAGTACT & TGACA A TGGT \\
\hline Lser.CR1A2.Con & CA TA TCCA T G & C TGA T GC T GA & A ACACCTTGG & C GA A C G G G G A & G T C G A G T A C T & TGACAA T G G T \\
\hline Lser8_2 & C A T A C C A A A C & GCAGCTTTTA & A GC TCGCAAT & C A G G A A A G T G & C C T A A A T T A T & T T GC C GACC T \\
\hline Lser6 & $-\cdots$ & $\cdots$ & $\cdots \cdots$ & $\ldots-\cdots$ & $\ldots$ & $\ldots-\cdots$ \\
\hline Lser1 & C A T ACCAA A C & GCAGCTTTTA & A GCTCGCAAT & C A G G A A A G T G & CC TAAA T TA $T$ & T T GCCGACC T \\
\hline Lser9_2 & C A TACCAAAC & GCAGCTTTTA & AGCTCGCAAT & C A G G A A A G T G & CC TAAA T TA $T$ & T T GCCGACC T \\
\hline Lser.CR1B2.Con & C A T A C C A A A C & GCA GC T T T T A & A GC TCGCAAT & C A G G A A A G T G & C C TAA A T TA $T$ & T T GC C G A C C T \\
\hline Lser6_2 & T G & A GC A & TAA T & CCC $\cdots$ A A T & G A A A A & G G T \\
\hline Lser7 & TTTTAACATG & TCAATAAGCA & GTCTGATAAT & ACC C - A A T & GCC TGGAAA A & GGGGTAGAGT \\
\hline Lser5 & TTTTAACATG & TCAATAAGCA & GTCTGATAAT & ACCC - - A A T & GCC TGGAAA A & GGGGTAGAGT \\
\hline Lser.CR1A1.Con & T T T T A A C A T G & TCA A T A A G C A & G TC TGATAA T & A C C C - - A A T & G C C T G G A A A A & G G G G T A G A G T \\
\hline Lser7_2 & TTTTAACCTG & A T GAGCA & G A T A A T & ACC $-\cdots$ A A T & C T G G A A A A & A G T \\
\hline Lser10_2 & C T T T A A & A T G A G C A & A T A A T T & A C C C - - A A T & G C C T G G A A A A & A G A G T \\
\hline Lser3_2 & C TTTAACCTG & TCAATGAGCA & GCCTGATAAT & ACС $\cdots$ A A T & GCC TGGAAA A & AGGGTAGAGT \\
\hline Lser.CR1A2.Con & T T T T A & GCA & TAA T & ACCC - - A A T & GC C T G G A A A A & G T \\
\hline Lser8_2 & G T T T A A C C A A & T G C T T G A G G G & A A T G TATT T T & C C C A - - G A C & C C C T G G A A G A & A A C A G A C A C T \\
\hline Lser6 & $\ldots$ & $\cdots-G$ & ГT T T T & C C C C A T GACC & A A G A & C T \\
\hline Lser1 & G T T TAACCAA & T GC TCG & T T T T & C C C A - - GAC & G A & C T \\
\hline Lser9_2 & G TT TAACCA A & T GC T T G & T T T T & CCCA - - G AC & G A & C T \\
\hline Lser.CR1B2.Con & G T T T A & TGC & AAGGTATTTT & C C C A - - GAC & C C C T G G & A A C \\
\hline Lser6_2 & T A & A G & 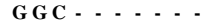 & G & & \\
\hline Lser7 & C A T C C & C A G & GGC $\ldots \ldots$ & $-\cdots \mathbf{G G} A$ & A G A G G G T C & A G \\
\hline Lser5 & C A T C C & C A G & GGC $\ldots \ldots$ & - - - G G $A$ & TC & A G \\
\hline Lser.CR1A1.Con & C A T C C C C A T A & C C A G & $\cdots \cdots$ & $\cdots-G$ & G G G T C & T A T T C T \\
\hline Lser7_2 & C A T C C C C A T A & A A A C C A G & GGAAG $\cdots \cdots$ & $\cdots$ - A ACGCG & G G G T C & A A T C C T $A$ \\
\hline Lser10_2 & C A T C C C GA T A & C C A G & AG - . - & $\cdots$ & G G G T C & A G \\
\hline Lser3_2 & C A T C C C G A T A & C T G A A A C C A G & G G & $\cdots-$ A A C GCG & A C A G A G G G T C & T T A C A G \\
\hline Lser.CR1A2.Con & C A TCCCCATA & C C A G & G - - - - & - - - - & A C A G A G G G T C & A A T \\
\hline Lser8_2 & G G T A C T A A T C & C A A A A A A C A A & A T A A A C C T A T & T G A T G A G C C G & TCGT $\cdots \cdots$ & $-\cdots$ C G T A C A G \\
\hline Lser6 & GGTACTAATC & C A A & C C T A T & T G G & T $\ldots \ldots$ & $-\cdots$ \\
\hline Lser1 & G G T A C T A A T C & C C A A A A C C A A & A TAA A C C T A T & T T T T G A & T C G T $\cdots \cdots$ & $\cdots \mathrm{C}$ \\
\hline Lser9_2 & GGTACTAATC & C A A & C C TA T & G G T - G A & G T $\ldots \ldots$ & $\cdots$ C G TACAG \\
\hline Lser.CR11 & GGTACTAATC & C C A A A & C T A T & T G T T G & - & AG \\
\hline Lser6_2 & G C C G A T T T C C & C T C C T G C C A C & C A G T C G C G A A & G A C T C T T G A G & G C T C T C C T C C & T C C C C C \\
\hline Lser7 & GCCGATTTCC & CTCCTGCCAC & G C G A A & GACTCTTGAG & GCTCTCCTCC & C C A G T T \\
\hline Lser5 & GCC C & A C & A A & A G & C C & T T \\
\hline Lser.CR1 & G C C G & C C A C & G A A & T T G A G & TCC TC C & G T T \\
\hline Lser7_2 & GCCGATTTCC & C TCC T G TCAC & C A GC C GCGA A & GACTCTTGTG & GCGC - . - & - C C C C C A GC T \\
\hline Lser10_2 & G C C G A T T T C C & C T C C T G TC A C & C A G T C G C G A A & G A G T C T T G A G & G C G C T C C T C C & TC C C C C A G C T \\
\hline Lser3_2 & GCCGATTTCC & C TCC TGTCAC & C G A A & GACTCTTGAG & GC TCC T C C & G C T \\
\hline Lser.CR1 & GC C & C A C & G A A & A G & C C & G C T \\
\hline Lser8_2 & A C C A A TA T G T & C TAC TGGACA & C A A T A G G GA A & A C TAC TGGAG & A A A A T A A T A T & A C A G TCGCT T \\
\hline Lser6 & ACС A A TA TGT & C TACTGGACA & C A A T A G G GA A & ACTACTGGAG & TA A T A T & ACAGTCGCT T \\
\hline Lser1 & A C C A A T A T G T & C T A C T G G A C A & C A A T A G G G A A & A C T A C T G G A G & A A A A T A A T A T & A C A G T C GC T T \\
\hline Lser9_2 & ACCAATATG T & C T A C T & G A A & A G & A T A T & ACAGTCGCTT \\
\hline Lser.CR1B2.Con & ACCAATATG T & C TAC T G GACA & C A A T A G G G A A & A C T A C T C & A T & ACAGTCGCT T \\
\hline Lser6_2 & - - G T C & A T C $\cdots$ C A & & $\ldots \ldots$ CGC & & \\
\hline Lser7 & - - G T C C A G T C & A T C $\cdots$ C A & G C A G C T - & $\cdots \cdots$ C GC & C A T C A T $\cdots$ & $\ldots \ldots$ \\
\hline Lser5 & - - G T C & A T C $\cdots-C$ A & C T - & $\cdots \cdots$ CGC & $\mathbf{T}-$ & - \\
\hline Lser.CR1 & - - G & T TC - - C C A & $\mathbf{T}-$ & $\cdots \cdots$ C GC & & \\
\hline Lser7_2 & - - G TCCTGCC & A T & GC TC & G - - C C C AC & T GGA T & TCCGTAAGAT \\
\hline Lser10_2 & - - G TCCTGCC & A T T $\cdots$ T T & A T A GCAGCTC & G - - C C C AC & I T GGA T & TCCGTAAGA T \\
\hline Lser3_2 & - - G T C C T GC C & A T C $\cdots$ T T & G C T C & G - . - C C A C & T G G A T & T C C G T A A A T T \\
\hline Lser.CR1 & - - G T C & A T $\mathbf{T} \cdots$ T A & T T & G - - - c & A T & G A T \\
\hline Lser8_2 & G T T & C A A G C G A & C T & G TC A A A C & $\mathbf{C} \mathbf{T}$ & G C \\
\hline Lser6 & G T T A C C & T GA A G C G A & TC T & G TCA A GC T AC & C T & $\mathbf{T} \mathbf{T}$ \\
\hline Lser1 & G T T A C C A A T C & G T T G A G G C G A & A A A A C G G TC T & G TC A A G C T A C & T A T G GC T & T T C G T A A A G C \\
\hline Lser9_2 & GTTACCAATC & G T TGA A GCGA & C G G T C T & GTCAAGCTAC & T GGC T & T TCG TA A A G C \\
\hline Lser.CR1 & G T T A C C A A T C & G T T G A A G C G A & A A A A C G G TC T & G TCA A GC T A C & C A A TA T G GC T & T T C G T A A A G C \\
\hline Lser6_2 & 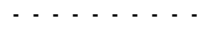 & $\cdots \cdots$ & $\cdots-\cdots$ & C C & A T C T & $\mathrm{CT} \mathbf{T}$ \\
\hline Lser7 & $\cdots \cdots$ & $\cdots \cdots$ & $\cdots \cdots$ TAAC & C G C C A T A A C C & A C T C A G A T C T & C A T A G G GCA T \\
\hline Lser5 & $\ldots \ldots$ & $\ldots \ldots$ & $\cdots \cdots$ TAAC & C GCCA T A A C C & AC TCAGATCT & CATAGGGCTT \\
\hline Lser.C & & & - T $\mathbf{A A A C}$ & $\mathrm{CC}$ & C T & $\mathbf{T T}$ \\
\hline Lser7_2 & & $\cdots-\cdots \mathbf{A}$ & & C C & C T & $\mathbf{T ~ T}$ \\
\hline Lser10_2 & GCA C A GCAC - & $\cdots \cdots$ C ACC T & AC & C C & G A T C T & G GC T T \\
\hline Lser3_2 & G C A C A G C A C - & $\cdots-$ C A C C $T$ & C A G C A T T A A C & C G C C A T A A C C & A C T C A G A T C T & C A C A A G G C T T \\
\hline Lser.CR1 & GCACAGCAC - & $\cdots-$ CACC T & C AGCA T TA A C & C GCCA T A A C C & AC TCAGA TC T & C ACA A GGCT T \\
\hline Lser8_2 & A A GA TCA A C C & I T A A C GCA A & C A T G G T & G A C G G A A A C A & G G & \\
\hline Lser6 & A GA T C A A C C & A T A GACGCA A & T A T G G T & C C A & $\mathbf{T} \mathbf{T}$ & C A A - - - \\
\hline Lser1 & A A G A T C A A C C & A T A G A C GC A A & T TCA TA T G G T & C A C G G A A A C A & G C A C A A A A G G & C A A $\cdots$ T A \\
\hline Lser9_2 & A A G A T C A A C C & A T A G A C G C A A & T TCA T A T G G T & C A C G G A A A C A & G C A C A A A A G G & C A A - - - T A \\
\hline Lser.CR1B2.Con & A A G A T C A A C C & A T A G A C G C A A & T TCA T A T G G T & C A C G G A A A C A & G C A C A A A A G G & C A A - - \\
\hline
\end{tabular}




\begin{tabular}{|c|c|c|c|c|c|c|}
\hline Lser6_2 & GACCA G - - & $\ldots \ldots$ & $\ldots \ldots$ & $\ldots \ldots$ & - - C CAGAC T T & A T C G A A G A C C \\
\hline Lser7 & A A A C C A G $\cdots$ & $\ldots$ & $\ldots$ & $\ldots$ & - C C AGACT T & ATCGAAGACC \\
\hline Lser5 & AA A C C A G - - & $\cdots \cdots$ & 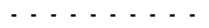 & $\ldots$ & - - C CAGAC T T & A TCGAAGACC \\
\hline Lser.CR1A1.Con & A A ACCAG - - & $\ldots \ldots$ & $\ldots \ldots$ & $\ldots \ldots$ & - - C CAGAC T T & A TCGAAGACC \\
\hline Lser7_2 & A A C C A GC A G & A GGCCTTGTG & A A A G G A C A C T & - - C C TAG TGG & C GC TAGAC T T & A T C G A A A G C C \\
\hline Lser10_2 & A A ACCAGCAG & AGGCCTTGTG & A A A GGACACT & - - C C TTGTGG & CGCTAGATTT & ATCGAAAGCC \\
\hline Lser3_2 & AAACCAGCAG & AGGCCTTGTG & AAAGGACACT & - C C TTGTGA & CGCTAGATTT & ATCGAAAGCC \\
\hline Lser.CR1A2.Con & AAACCAGCAG & AGGCCTTGTG & A A A GGACACT & - - C C T TGTGG & C GCTAGACT T & A TCGAAGGCC \\
\hline Lser8_2 & GA G G GCA A A G & GGGCAAACAA & AAAATACTGT & GCGATTATTA & CACTAGATGT & A A $-\mathbf{A} \mathbf{A} \mathbf{A C G C A}$ \\
\hline Lser6 & G A G G G C A A A G & G G G C A A A C A A & GAA A T A C T G T & GCGATTAT TA & C AC TAGA T G T & A A A A A ACGCA \\
\hline Lser1 & GA G G GCA A A G & GGGCAAACAA & GAAATACTGT & GCGATTATTA & CACTAGATGT & A A A A A ACGCA \\
\hline Lser9_2 & GA G G GCA A A G & GGGCAAACA A & GAAA TAC TG T & GCGATTATTA & CACTAGATGT & A A A A A ACGCA \\
\hline Lser.CR1B2.Con & G A G G GC A A A G & G G G C A A A C A A & GAA A T A C T G T & GCGATTA T T A & C AC TAGA T G T & A A A A A A C GCA \\
\hline Lser6_2 & T TC GA T ACA G & TAGCCA T A C & C ACA C T & C AGGACATCC & AGCAGTTCAC & C A T G \\
\hline Lser7 & T TCGATACA G & T TAGCCA TAC & C ACACTCTTC & C AGGACATCC & AGCAGTTCAC & C A T G \\
\hline Lser5 & T TCGATACA G & T TAGCCA TAC & C ACACTCTTC & CAGGACATCC & AGCAGTTCAC & CATGCCCAAC \\
\hline Lser.CR1A1.Con & T T C G A T A C A G & T T A GC C A T A C & C ACACTCT T C & C A G G A C A T C C & A GCA G T TCAC & C A T GCCCA A C \\
\hline Lser7_2 & T TCGA T A C A G & TCAGTCA TAC & C ACACTCT TC & C AGGACATCC & TGCAGTCCAC & C A T GCCCAAC \\
\hline Lser10_2 & T T C G A T A C A G & TCAGCCA T GC & C ACAC TC T TC & C A G GACA T C C & T GCA G T C C A C & C A T GCCCA A C \\
\hline Lser3_2 & T TCGA T ACA G & TCAGTCA T GC & C ACACTCTTC & C AGAACA TCC & TGCAGTCCAC & C A T G \\
\hline Lser.CR1A2.Con & T TCGA T ACA G & TCAGTCA TAC & C ACACTCT TC & C A G GACA TCC & T GCA G TCCAC & C A T GCCCAAC \\
\hline Lser8_2 & T T T A A C TC T G & C TAAC T G G G G & A T A T T T A & C A A A C TC T T C & GCA G AC T T G G & A G T A C C A G A T \\
\hline Lser6 & T T T A A C TC T G & C T A A C T G G G G & A T A T T A & C A A A C TC T T C & GCA G AC T T G G & C A G A T \\
\hline Lser1 & T T TA AC TC T G & C TAACTGGGG & TTTAATATTA & CAAACTCTTC & GCAGACTTGG & A G TACCAGAT \\
\hline Lser9_2 & T T T A A C T C T G & C T A A C T G G G G & T T T A A T A T T A & C A A A C TC T T C & G C A G A C T T G G & A G T A C C A G A T \\
\hline Lser.CR1 & T T A A C T C T G & C TA A C T G G G G & $\mathbf{T} \mathbf{T}$ T & C A A A C T & G C A G A & A G A T \\
\hline Lser6_2 & A & C G & & & G & \\
\hline Lser7 & A C T T G - A A A & A G A T G G A T T G & T G A A T T A T G T & G A G T T A C C G C & C A G T C & T T T A G \\
\hline Lser5 & A A C T T G - A A A & A G A T G G A T T G & T GAA T TA T G T & G A G T T A C C G C & C A G T C G T T C G & T A G A G T T T A G \\
\hline Lser.CR1A1.Con & A AC T T G - A A A & AGATGGATTG & Г G T & C G C & C A G T C & T T T A G \\
\hline Lser7_2 & T A C T T G - A A G & A GA T G GA T T G & TC T & G A G T G G C C G T & C A G T C A T T C G & T T G A G T T T A G \\
\hline Lser10_2 & T T G - A A G & I T G & C T & C G T & T C G & T T A G \\
\hline Lser3_2 & A A C T C G - A A G & A GA T G GA T T G & TAAA $T$ T A $\mathbf{T}$ C $T$ & G A G T G G C C G T & C A G T C G T T C G & T T GA \\
\hline Lser.CR1A & A A C T T G - A A G & A G A T G G A T T G & T A A A T T A T C T & G A G T G G C C G T & C A G T C G T T C G & T D G A G T T T A G \\
\hline Lser8_2 & TATTTGACTC & TGA T TA T TAG & T - A GCTACT T & C TCTAATAGA & AGACTGTGC - & TA T GACACAG \\
\hline Lser6 & T A T T T G A C T C & T GA T T A T T A G & $\mathbf{T}-\mathbf{A} \mathbf{G} \mathbf{C} \mathbf{T}$ & C T C T A A T A G A & A GAC T G T G C - & T A T G A C A C A G \\
\hline Lser1 & TA T T TGAC TC & T A G & $\mathbf{T}-$ & A T A G A & A G A C T & ICA G \\
\hline Lser9_2 & TA T T T GAC TC & T GA T TA T TA G & $\mathbf{T}-\mathbf{A} \mathbf{G} \mathbf{C} \mathbf{T}$ & C TC TA A T A G A & AGACTGTGC - & TA T GACACA G \\
\hline Lser.CR1I & TA T T TGACTC & TGATTATTAG & $\mathbf{T}-\mathbf{A}$ GC TAC T T & C TCTAATAGA & AGACTGTGC - & TA T G A C A C A G \\
\hline Lser6_2 & A G T G T & $\mathrm{CCC}$ & A & TC & & \\
\hline Lser7 & G A C A A G T G T & A A C C C & G & G G A G T A & C C & \\
\hline Lser5 & G A C A A G T G T & TC C A A A A C C C & G T A G A G T T A A & A C T A G G C G T T & C C C C A A & \\
\hline Lser.CR1A & I G A C A A G T G T & T C C A A A A C C C & G T A G A G T T A A & A C T A G G H G T H & C C T C A A & \\
\hline Lser7_2 & A G ACAAA T G T & TCCAAAACC C & G TAGAG T TAA & A C A G G GCG T C & C C T C A A & \\
\hline Lser10_2 & A G A C A A A T G T & T C C A A A A C C C & G T A G A G T T A A & A C A G G G T G T C & C C TC A A & \\
\hline Lser3_2 & G A C A A A T G T & T C C A A A A C C C & G T A G A G T T A A & A C A G G G C G T C & C C C C A A & \\
\hline Lser.CR1A & A GACAAA T G T & TCCA A A A C C C & G T A G A G T T A A & ACA A G G C G T C & C C T C A A & \\
\hline Lser8_2 & A A G A A G G T G T & - T A A G A T G C & A T A A G G T C A C & G G C A G G C G T - & & \\
\hline Lser6 & A G A A G G T G T & - TAA GA T G C & A T A A G G T C A C & G G C A G G C G T - & C C C C A A & \\
\hline Lser1 & A A G A A G G T G T & - T A A G A T G C & A T A A G G T C A C & G G C A G G C G T - & $\cdots \cdots$ & \\
\hline Lser9_2 & A A G A A G G T G T & - - TA A G A T G C & A T A A G G T C A C & G G C A G G C G T C & C C A C A A & \\
\hline Lser.CR1B2.Con & A A GA A G G T G T & - TAAGA T GC & A T A A G G T C A C & G G C A G G C G T - & $\cdots \cdots$ & \\
\hline
\end{tabular}

Supplemental Figure 2 Sequences obtained from $L$. sericata clones alongside consensus clusters 


\begin{tabular}{|c|c|c|c|c|c|c|}
\hline Cmac15 & A TCTCCATG & C TAATGCTGA & GAA TCT T T G & C G A G C G G G G A & G TAGA A T A T T T & TGACAATGGT \\
\hline Cmac1 & A A TCTCCA T G & C T A A T G C T GA & A A A A TCT TGG & C GA GC G G G G A & G T A G A A T A T T & TGACAA T GG T \\
\hline Cmac5 & А TC TCCATG & С TAATGCTGA & A A A ACCTTGG & C GAGCGGGGA & GTAGAATATT $T$ T & TGACAATGGT \\
\hline Cmac2 & A TCTCCATG & С TAATGCACA & А А А ТС T TGG & C GA GCGGGGA & GTAGAATACT & TGACAATGGT \\
\hline Cmac4 & А TС TСС А TG & СТАATGCTA & A A ACC TTGG & C GAGCGGGGA & GTAGAATACT & TGACAATGGT \\
\hline Cmac7 & A A TCTCCATG & C TAA TGCTAA & A A TACCTTGG & C GAGCGGGGA & G TAGAATAC T & TGACAA TGG T \\
\hline Cmac8 & AАTCTCCATG & С TAATGCTAA & A A A ACCTTGG & C GAGCGGGGA & GTAGAATACT & TGACAATGGT \\
\hline Cmac9 9 & GATCTCCATG & C TAA TGCTAA & A A A ACCTTGG & C GAGCGGGGA & G TAGAATACT & TGACAATGGT \\
\hline Cmac.CR1A.Con & А TC TCCATG & C TAA TGCTA A & A A A A C C T T G G & C G A G C G G G G A & G T A G A A T A C T & TGACA A T G G T \\
\hline Cmac15 & A T T TA A T C T G & T C A A T T A A G A & GC T T A G T A A T & A T GCC & T G & G TCA T \\
\hline Cmac1 & ATTTAACCTG & TCA A T TA A GA & GCTTAGTAAT & ACCCAACGCC & TGGAAA T C GG & GTAGAGTCAT \\
\hline Cmac5 & TTTTAACCTG & TCAA T TAGGA & GCTTGATAAT & ACCCAACGCC & TGGAAA T C GG & GTAGAGTCAT \\
\hline Cmac2 2 & TTTTAACCTG & TCAA T TAGGA & GCTTGATAAT & ACCCACCGCC & TGGA A A T C GG & GTAGAGTCAT \\
\hline Cmac4 & T T T T A ACC T G & TCAA T T A G G A & GCTTGATAAT & ACC C A A C GCC & T G GA A A T C G G & G T A G A G TCA T \\
\hline Cmac7 & T TTTA AC C TG & TCAATTAGGA & GCTTGATAAT & ACCCAACGCC & TGGGAA TCGG & GTAGAGTCAT \\
\hline Cmac8 & T T T TA AC C TG & TCA A T TAGGA & GCTTGGTAAT & ACCCAACGCC & TGGAAA TCGG & GTAGAGTCAT \\
\hline Cmac9 & ГTTTAACC TG & TCA A T TAGGA & GCTTGATAAT & ACCCAACGCC & TGGAAA TCGG & GTAGAGTCAT \\
\hline Cmac.CR & T T T T A A C C T G & TCA A T T A G G A & GCT T GA T A A T & A C C C A A C G C C & T G G A A A T C G G & G T A G A G TC A T \\
\hline Cmac15 & C CA A T T T T G & G A & G C G A A & T A & G T & $\mathbf{C} \mathbf{T}$ \\
\hline Cmac1 & С С A A T TTTG & A A ACCAGGAA & A A GACGCGAG & CGAGGGTTTA & TCA TACAGGC & CGATATCCTT \\
\hline Cmac5 & T T T T G & A A A C C AGGGA & A A GACGCGAG & Г T A & A G G C & CGATATCCCT \\
\hline Cmac2 & C TAATTTTG & A A ACCAGGGA & A A GACGCGAG & CGAGGGTTTA & TCGTACAGGC & CGATATCCCT \\
\hline Cmac4 & C CAATTTTG & A A A C C A GGGA & A A GACGCGAG & C GAGGGTTTA & TCGTACAGGC & CGATATCCCT \\
\hline Cmac7 & СССААТTTTG & A A ACCAGGGA & A A GACGCGAG & C GAGGGTTTA & TCGTACAGGC & CGATATCCCT \\
\hline Cmac8 & CССАATTTTG & A A ACCAGGGA & AAGACGCGAG & C GAGGGTTTA & TCGTACAGGC & CGATATTCCT \\
\hline Cmac9 & ССАA T T T TG & A A ACCAGGGA & A A GACGCGAG & C GAGGGTTTA & TCGTACAGGC & CGATATCCCT \\
\hline Cmac.CI & C C C A A T T T T G & A A A C C A G G G A & A A G A C G C G A G & C G A G G G T T T A & T C G T A C A G G C & C G A T A T C C C T \\
\hline Cmac15 & $\mathrm{C} \mathrm{A}$ & G A & G C & TC & T A A & C A T C T \\
\hline Cmac1 & C T TA T C A C C A & G TCG T GA A GA & C TCTTGAGGC & TCTTTTACTC & C T A A & A TCACCA TCT \\
\hline Cmac5 & C T T & G T C & A A G C & TC & T G A & A T C \\
\hline Cmac2 & C CA & G TCGCGAAGA & A A G C & T C & T GA & C A C C T \\
\hline Cmac4 & C T TATCACCA & GTCGCGAAGA & T T GA A G C & TC TACTC & C T A A & A TCA TCACCT \\
\hline Cmac7 & С TTATCACCA & GTCGCGAAGA & CTCTTGAAGC & TC TTC TAC TC & C C C C A GC TAA & ATCACCACCT \\
\hline Cmac8 & CTTATCACCA & G TCGCGAAGA & C TCTTGAAGC & TCTTCTACTC & C C C C A GC TGA & A TCACCACCT \\
\hline Cmac9 & C A C C A & G TCGCGAAGA & T G A A G C & $\mathrm{TC}$ & C T G A & C A C C T \\
\hline Cmac.CR & C T T A T C A C C A & G T C G C G A A G A & $\Gamma$ T G A A G C & TC T T C T & C T G A & A TCACCACC T \\
\hline Cmac15 & G C T & A C & C G & $T$ & $\mathrm{ACCA}$ & C A \\
\hline Cmac1 & G A G C T & C G T C A C C A G T & GA T T C C G & C A T & A G - - C & C A GCA T T A A C \\
\hline Cmac5 & G C A GC T & CG TCACCAGC & G A T T C C G & C A T & AGTACCACCA & C A G C \\
\hline Cmac2 & TATAGCAGC T & C G TCACCAGC & ATGGATTCCG & TAATATGCA T & AGTACCACCA & CAGCATTAAC \\
\hline Cmac4 4 & TA TAGCAGC T & CGCCACCAGC & ATGGATTCCG & TAA TA T GCA T & AGTACCACCA & C AGCATTAAC \\
\hline Cmac7 & TATAGCAGC T & $\ldots$ & $\ldots \ldots$ & $\ldots \ldots$ & $\ldots \ldots$ & $\ldots$ \\
\hline Cmac8 & TACA GCA GC T & CGTCACCAGC & ATGGATTCCG & TAATATGCAT & AGTACCACCA & C AGCATTAAC \\
\hline Cmac9 & T A T & C A G C & C G & A $\mathbf{T}$ & C A & C A G \\
\hline Cmac.CR & TA T A GC A G C T & C G T C A C C A G C & A T G G A T T C C G & TA A T A T GCA T & A G T A C C A C C A & T T A A C \\
\hline Cmac15 & Г С C & A G & $\mathbf{T} \mathbf{T}$ & G & T G & AC \\
\hline Cmac1 & GACCGTATCC & ACTCAAATAG & A T GGTTT & C A G & T G T G & A C A A T \\
\hline Cmac5 & TC C & A A A T A G & G T T G & G G & T G T G & A G C G \\
\hline Cmac2 & S A C G T A TC C & ACTCAAA T A G & C C C ACGGTTT & G A A C C A GCA G & A GACCGTGTG & A GCGA A C A A T \\
\hline Cmac4 & GACCGTATCC & ACTCAAATAG & C C CATGGTTT & GA A C C A GCA G & AGACCGTGTG & AGCGAACAAT \\
\hline Cmac7 & $\ldots$ & $\ldots$ & $\ldots$ & $\ldots \ldots$ & $\ldots \ldots$ & $\ldots \ldots$ \\
\hline Cmac8 & A C C GTATCC & АС TCAAATAG & G G T T T & C C A GCA G & G T G T G & AGCGAACAAT \\
\hline Cmac9 & T A TC C & A A A T A G & G T T T & A G & TG TG & CA A T \\
\hline Cmac.CR & GACCGTA T C C & AC TCA A A T A G & C C CA T GGTT T & G A A C C A G C A G & A G A C C G T G T G & A G C GAACA A T \\
\hline Cmac15 & $\mathrm{CA}$ & G T & & & T G & C A \\
\hline Cmac1 & G C A & A C C T GA & A GCA T T & G T C & AC T G & Г TC C A \\
\hline Cmac5 & G G C A & A C C T G T & C A T T & $\mathrm{TC}$ & A G & C A \\
\hline Cmac2 & C T T GTGGCA & A C C T G T & C A T T & TC & $\mathbf{A ~ G}$ & TACTCTTCCA \\
\hline Cmac4 & C CTTGTGGCA & C TTGACCTGT & CAAAAGCATT & TGACACGGTC & AGCCACACAG & TACTCTTCCA \\
\hline Cmac7 & $\ldots$ & $\ldots$ & $\ldots$ & $\ldots$ & $\ldots$ & $\ldots$ \\
\hline Cmac8 & C TTGTGGCA & CTTGACCTGT & C A A A A GCA $T$ T & TGACACGGTC & AGCCACACAG & TAC TC T TC CA \\
\hline Cmac9 & C T T T & C T G T & A T T T & TC & A G & TAC TCT TCCA \\
\hline Cmac.CR & C C T T G T G GCA & C T T GACC T G T & C A A A A G C A T T & T G A C A C G G T C & A GC C A Y A C A G & TACTCT TCCA \\
\hline Cmac15 & G G A C A T C C T G & C A G T C C T C C T & C A A A C T A & G A & T G A & Г T G A G \\
\hline Cmac1 & C T G & CAGTCCTCCT & A C T A & A A & Г G A & T T G A G \\
\hline Cmac5 & C T G & C TCC T & I C A A & G A & T G A & T GA G \\
\hline Cmac2 2 & A TCCCG & CAGTCCTCCT & C G A A C A A & G A G A & T G T G A & ACTATTTTAG \\
\hline Cmac4 & GGACATCCTG & CAGTCCTCCT & TGCCGAACA A & C AC GA A G A G A & TGGATTGTGA & ACTATTTGAG \\
\hline Cmac7 & $\ldots \ldots$ & $\ldots \ldots$ & $\ldots \ldots \ldots$ & $\ldots \ldots \ldots$ & $\ldots \ldots$ & $\ldots \ldots$ \\
\hline Cmac8 & GACA TCC TG & C AGTCCTCTT & TGCCGAACAA & C ACGA A G A GA & TGGA T TGTGA & ATTATTTGAG \\
\hline Cmac9 & GGACATCCTG & C AGTCCTCCA & TGC C GAACA A & A G A G A & T G A & I T G A G \\
\hline Cmac.CR1A.Con & GGACA TCC TG & CAGTCCTCCT & TGCCGAACA A & C A C GA A G A GA & TGGA T T G T GA & ATTATTTGAG \\
\hline
\end{tabular}




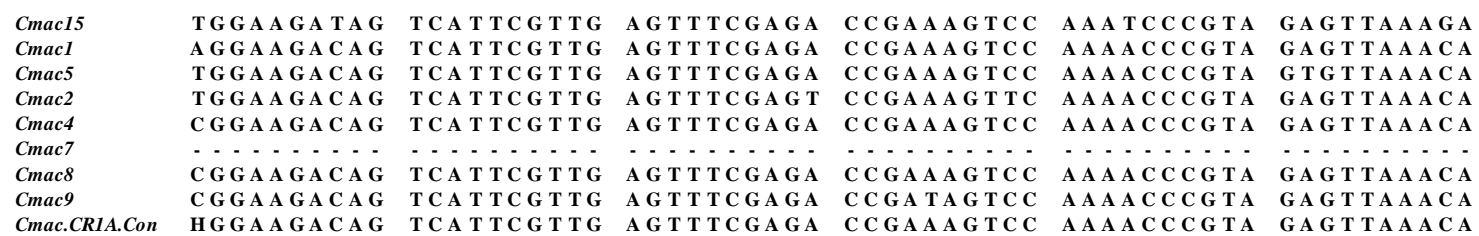

\section{Supplemental Figure 3 Sequences obtained from $C$. macelleria clones alongside consensus clusters}

\begin{tabular}{|c|c|c|c|c|c|c|}
\hline PReg28 & A TCT TCT TC & T G G G C C & GGGA T C & ССАAATGAGA & $\mathbf{T} \mathbf{T}$ & GAA \\
\hline PReg21 & TATCTTCTTC & TCC - TGGTCC & AGATGGGATC & C CAAA TGAGA & TAA TAACTTT & GGTTTGTGAA \\
\hline PReg18 & TATCTTCTTC & TCC-AGGGCC & GACGGGATC & CCAAATGAGA & ГАA TAACTTT & GGTTTGTGAA \\
\hline PReg7 & A TCTTCTTC & C C C CMGGAC C & GATGGGATC & C C A A A T GAGA & A A TAACTTT & GGTTTGTGAA \\
\hline PReg22r & TATCTTCTTC & TCC-CGGACC & GATGGTATA & T G A G G & ГT TA TAAAA T T & GGTAGGTGAG \\
\hline PReg27 & TA T C T TC T T C & TC C - A G G T C C & A G A C G G T A T A & C C C A A T G A G G & T T A T A A A A T T T & G G T A G G T G A G \\
\hline PReg31 & T A T C T T & TCC - AGGTCC & A GACGGTATA & T G A G G & TTATAAAAT T & I G G T G A G \\
\hline PReg8r & & $\ldots$ & 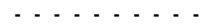 & $-\ldots$ & & $\cdots-\cdots$ GAG \\
\hline PReg29 & TATCTTCT TC & TCC - TGGAC C & A C GGACTC & G G T & A A A C $\mathbf{T}$ & A A T T T T $\cdots$ \\
\hline PReg17 & T A & TC C - T & C T C & & C T & $T \ldots$ \\
\hline PReg20 & TC & TCC - G & TC & & C T & T - - \\
\hline Preg.Cr1 & C T T C & TC C - K C & C T C & T C A & AC T & T T - - \\
\hline$P R$ & G & A & & & G & TACC $\cdots \cdots$ \\
\hline PReg22r & C G & G T & T T T & C C & C C G G & T A TC $\ldots$ \\
\hline PReg27 & C G & G T & T T T & C C & G G & TA T C $\cdots \cdots$ \\
\hline PReg31 & C G & G T & $\mathbf{T} T$ & & G G & $C \ldots$ \\
\hline PReg8r & A T A T G & G T & Г T T & & C C G G & TA T C $\ldots \ldots$ \\
\hline PReg12r & C G & G T & Г T T & & G G & T T T C $\cdots$ \\
\hline Preg.Cr1I & A T A T G GC C C G & G T & T T T & C C & C C G G & C - . - \\
\hline PReg29 & $\cdots$ TA & C T & $\mathbf{T} \mathbf{T}$ & T T & T G & GAC T T G \\
\hline PReg17 & $\ldots \ldots$ & C T & $T T$ & T T & [A G T G & G AC T T G \\
\hline PReg20 & $\cdots \cdots$ TA & C T & T T & & & C T T G \\
\hline Preg.Cr1 & $\cdots$ TA & C T & T T & $\mathbf{T} A$ & T G & C T T G \\
\hline & $\mathbf{T}$ & & & & & \\
\hline PReg3 & $-\ldots-\cdots$ & A T $\mathbf{T}$ & C A & T T & C - - T A & A A G G C G \\
\hline PReg21 & $\cdots \cdots$ & $\mathbf{A} \mathbf{T}$ & C A & & $\cdots \mathbf{T A}$ & G G C G \\
\hline PReg29 & $\mathrm{A} T$ & C T & TC & & T T C & \\
\hline PReg17 & $\mathbf{T}$ & $\mathbf{T}$ & T C & & TC & \\
\hline PReg20 & C TC T A A A T A T & C T & $\mathrm{ATC}$ & T T & T T C & A T G A A \\
\hline Preg.Cr1C.Con & C TCTAAATAT & C C ACAAGCC T & GGAAAATATC & A A GA $\cdots$ G T T & T TGCCCATTC & CCAAAATGAA \\
\hline & & & & & & \\
\hline$P R$ & & & T A & & & \\
\hline PReg21 & $\mathbf{C} \mathbf{T}$ & C T & $\mathbf{T A}$ & T G & A C & A C \\
\hline PReg18 & GAAACCC C T & C T & T A & T G & A C & TGGAA \\
\hline PReg7 & C C T & C T & $\mathbf{T A}$ & T T G & A A C & G T G G A A \\
\hline Preg.Cr & & & & & & \\
\hline PReg22r & & & T A & & & A A \\
\hline PReg27 & & C T & T A & & & A G G A A \\
\hline PReg31 & $T T$ & C T & $\mathrm{T} \mathrm{TA}$ & & $\mathbf{A} \mathbf{T}$ & A GGAA \\
\hline PReg8r & $\mathrm{TT}$ & C C T & A T A & TTTG & T G T T G G A T & T T A G G A A \\
\hline PReg12r & & & & & & \\
\hline & & & T A & & & A A \\
\hline & & & & & & \\
\hline PReg17 & C A & C T T $\cdots$ T G T & [ A T C & A T C T G & T T T T T & T G T T C T A \\
\hline PReg20 & C A & C TC - - T T T & А T A TC & I TCTG & T T G T A T C T & G TA T G T TC TA \\
\hline Preg.Cr & C A & Г G T & $\mathrm{ATC}$ & T G & A T C T & G T T C T \\
\hline
\end{tabular}




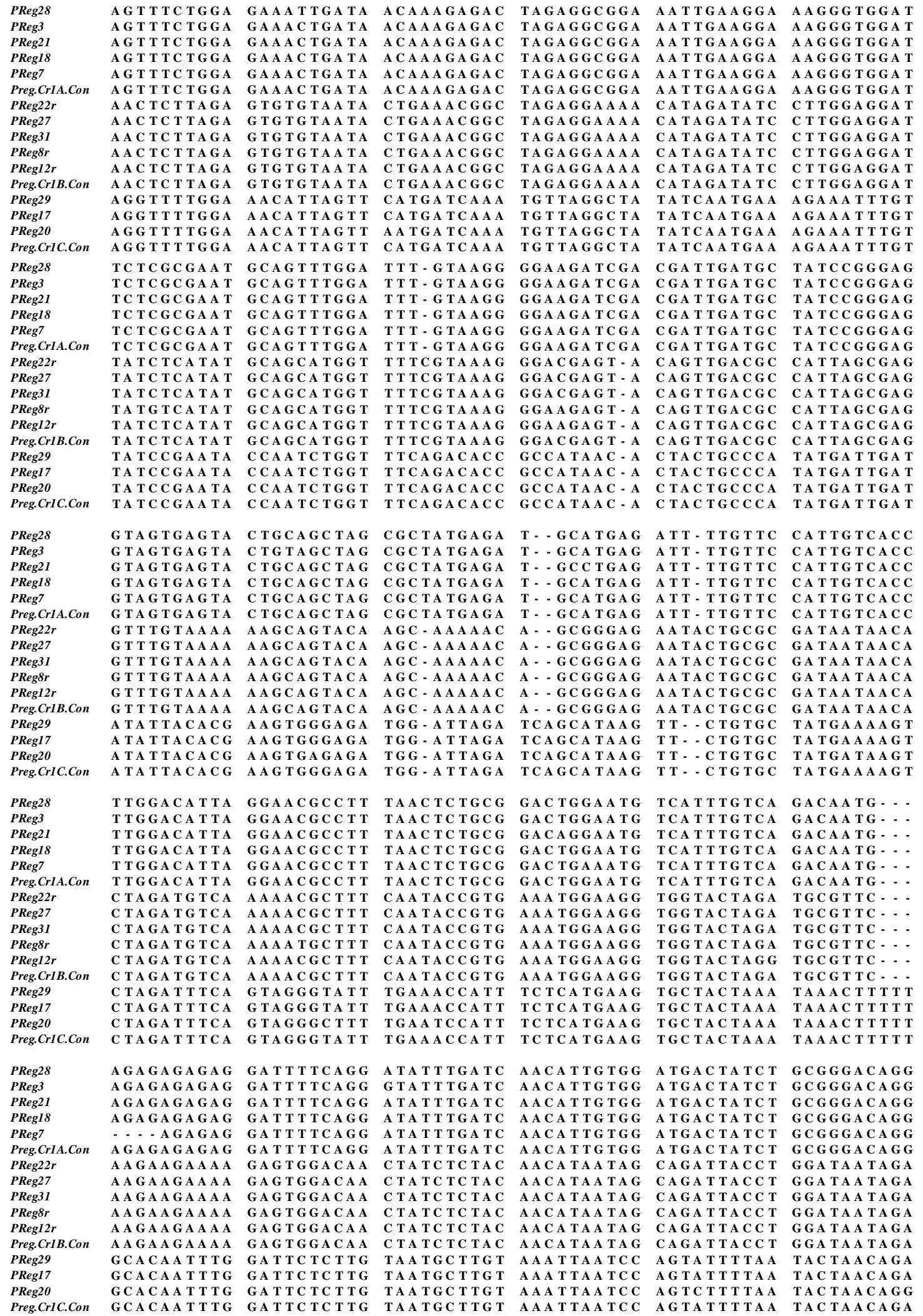




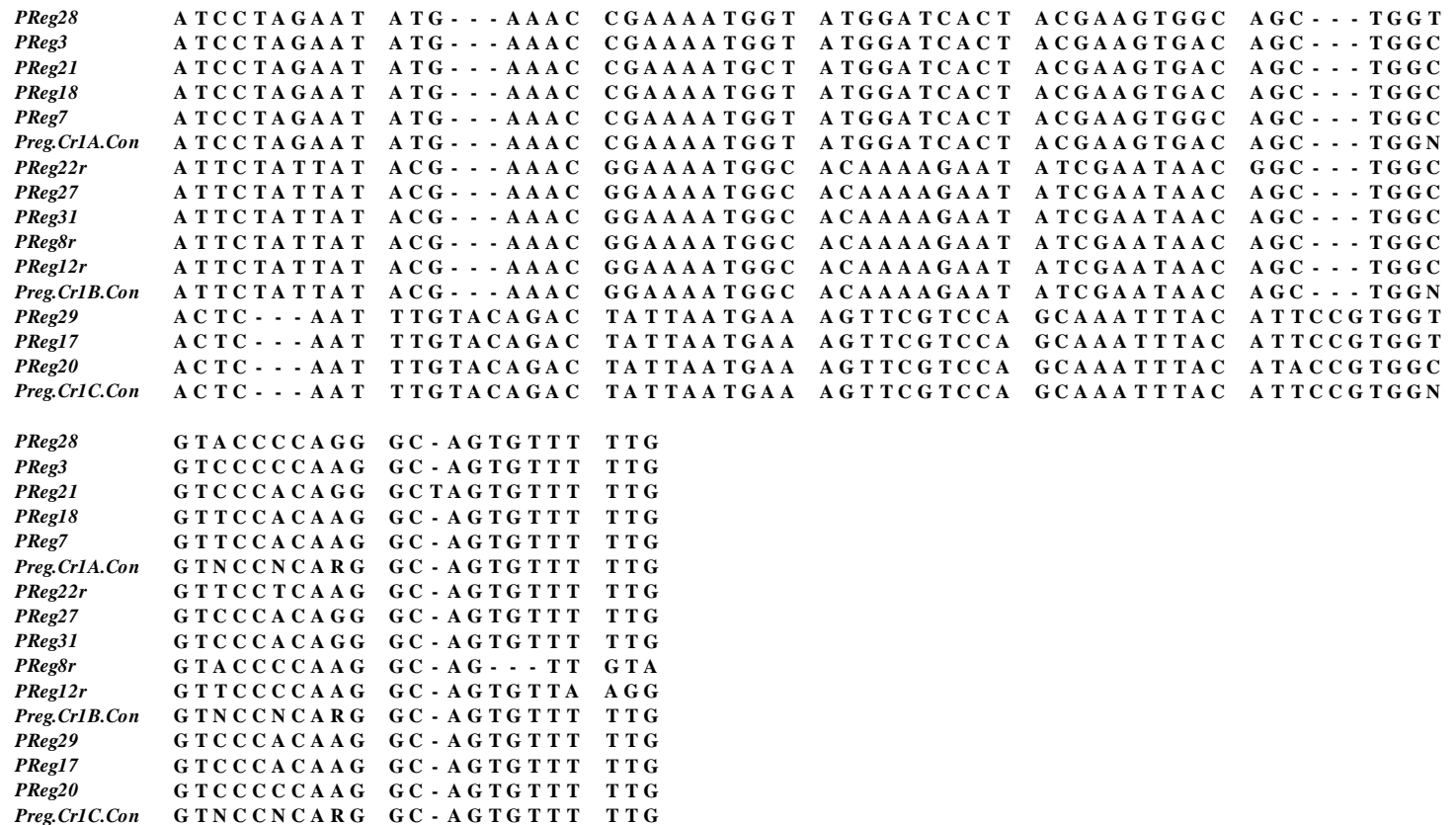

Supplemental Figure 4 Sequences obtained from $P$. regina clones alongside consensus clusters

\begin{tabular}{|c|c|c|c|c|c|c|}
\hline Sbul18 & C T C A A & T T A & C T A T & GCA I & $\mathbf{T}-$ & - - G \\
\hline Sbul2 & GATCCCAAAC & C GGGCACT TA & AGGTGGCTAT & C A GGCATAGA & C CGGAT $-\mathbf{A} \mathbf{T}-$ & - - GA TCGCCA \\
\hline Sbul23 & A TCCCAAAC & CGGGCACT TA & GGTGGCTGT & A A GCATAGA & C CGGAT - A T - & - - GA TCGCCA \\
\hline bul.CR1A.Con & A TССС A A AC & CGGGCACTTA & GGTGGCTAT & A GGCATAGA & C CGGAT - A T - & - - GA TCGCCA \\
\hline Sbul27 & GGTTCTTAGC & - AGTATTTT & A A AC ACC - & & & \\
\hline Sbul12 & GGTTCCTAGC & - A A G TA T T T T T & A A ACACCA T & TC - TCAATTA & CTGAGTAACT & C C A \\
\hline Sbul13 & GGTTCCTAGC & - A A G TATTTTT & AA ACACCA T & TC - TCAATTA & C TGAGTAAC T & C C A T \\
\hline Sbul24 & GGTTCCTAGC & - A G TA T T T T T & A A ACACCA T & TC - TCAAC TA & C T GAGT $-\mathbf{A} \mathbf{C} \mathbf{G}$ & CCATTAACCT \\
\hline Sbul28 & & & & & & \\
\hline Sbul29 & G G T & - A G T A & C C A T & $\mathbf{T C}-\mathbf{T}$ & $\mathbf{G} \mathbf{T}-\mathbf{A}$ & C C A \\
\hline SBul11 & GTTCCTAGC & - AGTATTTT & C C A T & TC - TCA A T TA & C TGAGT-AT T & C C A \\
\hline SBul5 & C T & - - A & C C A T & T A & $\mathbf{G} \mathbf{T}-\mathbf{A} \mathbf{T} \mathbf{T}$ & C A \\
\hline Sbul22 & G G T & T T T & C C A T & TC & $\mathbf{G} \mathbf{T}-\mathbf{A} \mathbf{T} \mathbf{T}$ & C A \\
\hline Sbul26 & C & $--A$ & C A T & Г A & $\mathbf{G} \mathbf{T}-\mathbf{A} \mathbf{T} \mathbf{T}$ & C C - \\
\hline Sbul25 & G G T & $--\mathbf{A}$ & C C A T & TC - TCA & $\mathbf{G} \mathbf{T}-\mathbf{A} \mathbf{T} \mathbf{T}$ & C C A \\
\hline Sbul32 & GGTTCCTAGC & - A A G TA T T T T T T & C C A T & TC - TCAATTA & $\mathbf{A} \mathbf{G} \mathbf{T}-\mathbf{A} \mathbf{T} \mathbf{T}$ & C C A \\
\hline Sbul15 & G T TCC TAGC & - A A T TA T T T & C C A T & TC - TCAATTA & C TGAGT-AT T & C C A \\
\hline Sbul3 & $\mathbf{T}$ & $--A$ & $\mathbf{T}$ & T C - & $\mathbf{T}-\mathbf{A} \mathbf{T} \mathbf{T}$ & C C A \\
\hline Sbul7 & c & - - & $\mathbf{T}$ & A & $\Gamma \mathbf{T}$ & C T \\
\hline Sbul17 & C & $--A$ & $\mathbf{T}$ & A & T T & C C \\
\hline SBul10 & T T C C T & - A A G TATTTTT & C C A T & TC - TCAATTA & $\mathbf{A} \mathbf{G} \mathbf{T}-\mathbf{A} \mathbf{T} \mathbf{T}$ & C C A T T T \\
\hline Sbul30 & GGTTCCTAGC & - A A T TATTTT & C C A T & TC - TCAATTA & $G T-A T T$ & C C A T T T \\
\hline SBul14 & GGTTCCTAGC & $-\quad$ A G T A T T T & C C A T & TC - TCAATTA & C T GAGT-A T T & T A A C C T \\
\hline SBul21 & G G T T C C T A G C & - A G TA T T T T T & A A A A C A C C A T & TC - TCAATTA & $\mathbf{C} \mathbf{T} \mathbf{G A G} \mathbf{T}-\mathbf{A} \mathbf{T} \mathbf{T}$ & C C A T T A A C C T \\
\hline Sbul4 & C T A G T & $--\mathbf{A}$ & C C A T & TC & $\mathbf{G} T-\mathbf{A} T \mathbf{T}$ & C C A \\
\hline Sbul & T T C C T A G C & $-\mathbf{A}$ & C C A T & T A & T T & C C A \\
\hline & C & $--A$ & C A T & Г A & A T T T & C C A \\
\hline Bul6 & & & & & & \\
\hline Sbul.CR1B.Con & GGTTCCTAGC & A111 & & TC - TCAATTA & $\mathbf{T}-\mathbf{A} T \mathbf{T}$ & C CATTAACC \\
\hline
\end{tabular}




\begin{tabular}{|c|c|c|c|c|c|c|}
\hline Sbul18 & ATGTGTTCAA & CAAGTGTTTT & GTCGAGGGTA & TTTTTCCACG & TAGATGGA A G & AAGCAAAAAT \\
\hline Sbul2 & TTGTGTTCAA & CAAGTGTTTT & G TCGAGGGTA & TTTTTCCACG & TAGATGGAGG & AAGCAAAAAT \\
\hline Sbul23 & ATGTGTTCAA & CAAGTGTTTT & GTCGAGGGTA & TTTTTCCACG & TAGATGGA A G & AAGCAAAAAT \\
\hline Sbul.CR1A.Con & ATGTGTTCAA & CAAGTGTTTT & G TCGAGGGTA & T T T T TC C ACG & TAGATGGA A G & A A GCAAAA \\
\hline Sbul27 & $\cdots$ - $\mathbf{A}$ T T TAA & C TAATCGCTT & A A A TTGGGCT & ATTTTCCTTC & C TTTTGGA - - & $\cdots$ A ACAAT \\
\hline Sbul12 & ITTATTCA A & C A A A T C GC T G & A A T T G GGCT & A T T T TC C T TC & C T T T T GGA - - & $\ldots \ldots$ A A A A T \\
\hline Sbul13 & ТТТТАТТСАА & CAAATCGCTG & A $\mathrm{AT}$ TGGGC T & ATTTTCCTTC & CTTTTGGA - - & $\cdots$ A ACAA T \\
\hline Sbul24 & АТTTATTCAA & CAAATCGCTT & AAATTGGGCT & ATTTTCCCTC & CTTTTGGA - - & $\cdots$ A ACAAT \\
\hline Sbul28 & $\ldots$ & $\ldots$ & $\ldots$ & $\ldots$ & $\ldots$ & $\ldots$ \\
\hline Sbul29 & ATTTATTCAA & CAAATCACT T & A A A TTGGGCC & ATTTTCCCTC & C TTTTGGA - - & $\cdots$ A ACA $\mathbf{A}$ T \\
\hline SBul11 & АТTTATTCA A & CAAATCACT T & A A A T TGGGCC & ATTTTCCCTC & C TTTTGGA - & $\cdots$ A A C A A T \\
\hline SBul5 & ТTТТАТТСАA & CAAATCACT T & A A TTGGGCC & ATTTTCCCTC & C TTTTGGA - - & $\cdots$ A ACAAT \\
\hline Sbul22 & ТTТТАТТСАА & CAAATCACT T & A A A T TGGGCC & ATTTTCCCTC & C TTTTGGA - - & $\cdots$ A ACAAT \\
\hline Sbul26 & ATTTATTCAA & TAAA TCACT T & A A A C TGGGCC & ATTTTCCCTC & CTTTTGGA - - & $\cdots-$ A ACAA $\mathbf{T}$ \\
\hline Sbul25 & TTTATTCAA & TAAA TCACT T & A A A C TGGGCC & ATTTTCCCTC & CTTTTGGA - - & $-\cdots A$ \\
\hline Sbul32 & T T TA T TС A A & TAAATCACT T & AAACTGGGCC & ATTTTCCCTC & C TTTTGGA - - & $\ldots-A$ \\
\hline Sbul15 & TTTATTCA A & TAA A TCACT T & A A ACTGGGC C & ATTTTCCCTC & C TTTTGGA - - & $\ldots-1$ \\
\hline Sbul3 & ТТТАТТСАА & TAA A TCACT T & A A ACTGGGCC & ATTTTCCCTC & CTTTTGGA - - & $\cdots$ A ACAAT \\
\hline Sbul7 & ATTTATTCAA & TAAA TCACT T & AAAC TGGGCC & ATTTTCCCTC & C T T T T GGA - - & $\cdots-A$ \\
\hline Sbul17 & ATTTATTCAA & CAAATCACT T & A A A C TGGGCC & ATTTTCCCTC & CTTTTGGA - - & $\cdots-A$ \\
\hline SBul10 & Т ТTA Т ТС А А & С ААA TCACT T & A A A T T T G & A T T T T TC & C TTTTGGA - - & $\ldots-1$ \\
\hline Sbul30 & T T TA T TCA A & CAAATCACT T & A A T T G & ATTTTCCCTC & C T T T T & $\ldots$ \\
\hline SBul14 & TTTATTCA A & CAAATCACT T & A A T T G & TC C C T C & C TTTTGGA - - & $\ldots-1$ \\
\hline SBul21 & A T T TATTCAA & C A A A TCAC T T & A A A T T G GGC C & A T T T TCCCTC & C T T T T GGA - - & $\cdots-A$ \\
\hline Sbul4 & ATTTATTCAA & A TCACT T & A A A T TGGGCC & ATTTTCCCTC & C TTTTGGA - - & $\cdots-1$ \\
\hline Sbul9 & T T T A T TCA A & A TCACT T & A A T T G G GC C & A TTTTC & C T T T T GGA - - & $-\ldots$ \\
\hline Sbul31 & TTTATTCA A & CAAATCACT T & A ATTGGGCC & ATTTTCCCTC & C TTTTGGA - - & $\ldots-A$ \\
\hline SBul6 & TTTATTCA A & A T C AC T T & A T TTGGGCC & C C TC & C TTTTGGA - - & $\ldots$ \\
\hline Sbul.CR1B.Con & A T T T A T TCA A & C A A A TCAC T T & A A A T T GGGC C & C C C T C & C T T T T G GA - - & $-\cdots$ \\
\hline Sbul18 & 8 & $\cdots$ & G T A & T A & & TCG \\
\hline Sbul2 & TGGTGCTCA T & TC C A $\cdots \cdots$ & ACCTGGTA & A A C C T C & T GA A C & T C G T \\
\hline Sbul23 & TGGTGCTTAT & TC C A $\cdots \cdots$ & A ACCTGGTA & A ACC TCCAGG & TGAACCA TCG & TC G T A \\
\hline Sbul.CR1 & TGG T TC TCA T & $\cdots$ & A ACC TGGTA & A A C C TC C A G G & T GA A C C A T C G & T C G T \\
\hline Sbul27 & TA T T TA TAA $T$ & C T G T T T & C - - & $\mathbf{T}-\mathbf{T} A$ & TC A & A A $\mathbf{T} \mathbf{T}$ \\
\hline Sbul12 & CATTTATAAT & C T G T T T & G - - & - A T A T A & T A A & A A $\mathbf{T}$ T \\
\hline Sbul13 & A TTTATA $T$ T $T$ & TCCACTGTTT & A T C G G G - - & - A T $\mathbf{T A T}$ & TGATATTTAA & A A $\mathbf{T} \mathbf{T}$ \\
\hline Sbul24 & САТTTATA & TCCATTGCTT & A T C GGG - - & - A TC T & TGATATTTCA & AАTTATAGAG \\
\hline Sbul28 & $\cdots \cdots$ & $\cdots \cdots$ & A T C G G G - - & - A TCTAAAGC & TGA TA TC TCA & A A T TATAGAG \\
\hline Sbul29 & ATTTATAAT & TCCACTGTTT & T C G G G - - & TC T A & ITATCTCA & A A T T T \\
\hline SBul11 & А T T TA TAA T & C T G T T T & G - - & $-\mathbf{A} \mathbf{T C} \mathbf{T} f$ & TC A & A A $\mathbf{T}$ \\
\hline SBul5 & A TTTATAAT & TCCACTGTTT & A T C G GG - - & - A TCTA & TGATA TCTCA & A A $\mathbf{T} \mathbf{T}$ \\
\hline Sbul22 & CATTTATAAT & TCCACTGTTT & AAA TCGGG - - & - A TCTAAAGC & TGATA TCTCA & A A TTATAGAG \\
\hline Sbul26 & A T T TA TAA T & TCCACTGTTT & A T C G G G - - & $-\mathbf{A} \mathbf{T C} \mathbf{T} f$ & TGA TA TC TCA & AATTATAGAG \\
\hline Sbul25 & A TTTATAAT & C T G T T T & A T C G G G - - & $-\mathbf{A} \mathbf{T C} \mathbf{T}$ & T G A T A & Г A G A G \\
\hline Sbul32 & A T T T A TA A T & C T G T T T & G T C G G G - - & $\mathbf{C} \mathbf{T} A$ & TCA & A A T T \\
\hline Sbul15 & ATTTATAAT & C AC T G T T T & A TCC & $-\mathbf{A T C} \mathbf{T}$ & T GA T A & A A $\mathbf{T} \mathbf{T}$ \\
\hline Sbul3 & САTTTATA & TCCACTGTTT & A A TCGGG - - & - A TCTA & TGATATCTCA & AАTTATAGAG \\
\hline Sbul7 & C A T T T A T A A T & TC C AC TG T T T & AAA TCGGG - - & - A TC TAAA G C & TGA TA TC TCA & A A T TATAGA G \\
\hline Sbul17 & A T T TA T A A T & TC TAC TG T T T & A T C G G G - - & A G C & T GA T A & A G A G \\
\hline SBul10 & T T TA T A A T & C A C T G T T T & A TCGGG - - & $-\mathbf{A} \mathbf{T C} \mathbf{T}$ & TCA & A A $\mathbf{T} \mathbf{T}$ \\
\hline Sbul30 & A TTTATAAT & TCCACTGTTT & AAA TCGGG - - & - A TCTAAAGC & TGATA TCTCA & A A T T T \\
\hline SBul14 & CATTTATAAT & TCCACTGTTT & AAA TCGGG - - & $-\mathbf{A} \mathbf{T C} \mathbf{T}$ & TGATATCTCA & A A T T T \\
\hline SBul21 & C A T T T A T A A T & TC C A C T G T T T & A A A T C G G G - - & - A TC TAAAGC & T G A T A T C TCA & A A T T A T A G A G \\
\hline Sbul4 & А T T TA TA A T & TC C A C T G T T T & A A A T C G G G - - & - A TC TAAAGC & T G A T A T C A C A & A A $\mathbf{T} \mathbf{T}$ \\
\hline Sbul9 & A TT TATAAT & TC C A C T G T T T & AAA TCGGG - - & - A TCTAAAGC & TGA T A TC TCA & A A $\mathbf{T} \mathbf{T}$ \\
\hline Sbul31 & T T T A T A A T & T C C A C T G T T T & T C G G G - - & ГС T & C TCA & A A T T T \\
\hline SBul6 & CATTTATAAT & TCCACTGTTT & AAA TCGGG - & - A TCTAAAGC & TGATA TC TCA & A A T TATAGAG \\
\hline Sbul.CR1B.Con & CATTTATAAT & TCCACTGTTT & AAA TCGGG - - & - A TCTAAAGC & TGA TA TC TCA & A A T T \\
\hline
\end{tabular}




\begin{tabular}{|c|c|c|c|c|c|c|}
\hline Sbul18 & C CATCTGCCT & ACTTGACACG & ATGGGAAAA & TACTTTAGAG & GATAGTATAC & AATCGACTGC \\
\hline Sbul2 & C CA TCTGCCT & ACT TGACACG & ATGGGAAAA & TACTGGAGAG & GATAATATAC & AATCGACTGC \\
\hline Sbul23 & C CATCTGCCT & ACT TGACACG & ATGGGAAAA & TACTGGAGAG & GATAGTATAC & A A TCGACTGC \\
\hline Sbul.CR1A.Con & C CA TCTGCCT & ACTTGACACG & A TGGGAAAA A & TACTGGAGAG & GATAATATAC & AATCGACTGC \\
\hline Sbul27 & GA A T T TC TAA & AC TCA ACACA & A TACCGAA $\mathbf{T}$ & TA T T TGAAA A & ACTCTTATGC & GATACACTG T \\
\hline Sbul12 & GA A T T GCT T A & A A TCAACAC T & A T ACCCAAA T & TA T T TGAAA & ACTCTTATGT & GATACACTGT \\
\hline Sbul13 & GA A T T GCT T A & A TCAACACT & TACCCAA A T & TA T T TGAAA & ACTCTTATGT & ICTGT \\
\hline Sbul24 & GAATTGCTAA & ACT TAACACA & TA TC GA A GC & TATTTGAAA & ACTCTTATGT & GATACACTGT \\
\hline Sbul28 & GAATTGCTAA & ACT TAACACA & ATACCGAAAT & TA T T TGAAAA & ACTCTTATGT & GATACACTGT \\
\hline Sbul29 & GAA T TGCTAA & AC T T A A C ACA & ATACCGAAAT & TA T T TGAAAA & ACTCTTATGT & GATACAGTGT \\
\hline SBul11 & GA A T T GC TA A & AC T TAACACA & A TACCGAA $\mathrm{T}$ & TA T T TGAAA A & ACTCTTATGT & GATACAGTGT \\
\hline SBul5 & Г T GC T A A & ACT TAACACA & C GAA A T $\mathbf{T}$ & TA T T TGAAA A & AC T & G T G T \\
\hline Sbul22 & GA A T T GC T A A & AC T T A ACACA & A T ACC GAAA T & TA T T TGAAA & ACTCTTATGT & GATACAGTGT \\
\hline Sbul26 & GAATTGCTAA & ACT TAACACA & ATACCGAAAT & TA T T TGAAA A & ACTCTTATGT & GATACACTGT \\
\hline Sbul25 & GA A T T GC TA A & ACT T A A C ACA & A TACCGAAA T & TA T T TGAAA A & ACTCTTATGT & GA T ACACT T T \\
\hline Sbul32 & GA A T T GC TA A & ACT T A A C ACA & A T ACC GAAA T & TA T T TGAAA A & ACTCTTATGT & GA TACACT T T \\
\hline Sbul15 & GAATTGCTAA & ACT TAACACA & ATACCGAAAT & TA T T TGAAA A & ACTCTTATGT & AC T G T \\
\hline Sbul3 & GAATTGCTAA & C T TAACACA & ATACCGAAAT & TATTTGAAA & T A T G T & C T G T \\
\hline Sbul7 & GA A T TGCTA A & ACT TAACACA & ATACCGAAAT & TA T T TGAAA A & ACTCTTATGT & GATACACTGT \\
\hline Sbul17 & GA A T T GC TAA & AC T T A A C AC A & A T ACC GAAA T & TA T T TGA A A A & ACTCTTATGT & GA T A C A C T G T \\
\hline SBul10 & GA A T T GC TA A & ACT TAACACA & A TACCGAA $\mathbf{T}$ & TA T T TGAAA A & ACTCTTATGT & GATACACTG T \\
\hline Sbul30 & GA A T T GC TA A & AC T TAACACA & A T $\mathbf{T}$ & TA T T TGAAA A & T A T G T & C T G T \\
\hline SBul14 & GA A T T GC TA A & ACT T A ACACA & A T ACC GAAA T & TA T T TGAAA & ACTCTTATGT & GATACACTG T \\
\hline SBul21 & T T GCTA A & ACT TAACACA & A T & $\mathbf{T A}$ & AC T & GATACACTG T \\
\hline Sbul4 & GA A T T GC T A A & ACT T A A C ACA & A T ACC GAAA T & TA T T T GAAAA & ACTCTTATGT & GA TACACT T T \\
\hline Sbul9 & GA A T T GC TAA & ACT T A A C ACA & A T $\mathbf{T}$ & TA T T TGAAA A & T A T G T & ICTGT \\
\hline Sbul31 & GAATTGCTAA & ACT TAACACA & ATAC & TA T T TGAAA A & TA T G T & ICTGT \\
\hline SBul6 & GAA T T GCTAA & AC T TAACACA & ATACCGAAA T & TA T T TGAAAA & ACTCTTATGT & GATACACTGT \\
\hline Sbul.CR & GA A T T GC T A A & A C T T A A C A C A & A $\mathbf{T}$ & $\mathbf{T} \mathbf{T} \mathbf{T G}$ & TA T G T & C T G T \\
\hline Sbul18 & G T & G A & & C A & $\Gamma \mathrm{T}$ & G A C \\
\hline Sbul2 & T GACACA & G A & $\mathbf{C} \mathbf{T}$ & C G G A C C T & A T A & G A A C \\
\hline Sbul23 & TGACACA T G T & A G G C G A G A & TC T & C GGACC TGCA & A T T T T & G G GA C \\
\hline Sbul.CR & TGACACA T G T & C G A G G C G A G A & Г C T & C GGACC TGCA & A T T T T & G GAA C \\
\hline Sbul27 & $\cdots$ - C A T T T & C A A G C A T C G T & C C A T A T T A T C & T GCA TC T - C A & GCA T G G T T T C & T G T A A G TC TC \\
\hline Sbul12 & $\cdots-C$ CA & G T T & TC & TGCA TAC - CA & T T C & TCTC \\
\hline Sbul13 & $\cdots$ - CA T T T & $\mathbf{G} \mathbf{T}$ & TC & TGCA T A C - C A & GCA & TCTC \\
\hline Sbul24 & $\cdots$ - CA T T T & C A A GCA T C G T & A TC T TATC & TGCATCT-CA & GCC TGGTTTC & TG TAAG TC TC \\
\hline Sbul28 & $\cdots$ - CA T T T & C A A GCA TCG T & C C A TC T TA TC & TGCA TCT - C A & GCATGGTTTC & TG TAAGCC TC \\
\hline Sbul29 & $\cdots-$ CA T T T & C A A G C A T C G T & C T T A T C & TGCA TC T - C A & GCA T G G T T T C & T G TAAG TC T C \\
\hline SBul11 & $\cdots-$ CA T T T & C G T & T C & TGCA TC T - C A & G T T T C & TCTC \\
\hline SBul5 & $\cdots-C$ C A & G T & TC & TGCA TC T - C A & T T T C & TCTC \\
\hline Sbul22 & $\cdots$ - CA T T T & CA A GCA TCG T & А ТСТT ТА ТС & TGCA TCT - C A & G T T T C & TGTAAGTCTC \\
\hline Sbul26 & $\cdots$ - CA T T T & C A A GCA TCG T & A T C & TGCA TCT - CA & GCATGGTTTC & T G T A \\
\hline Sbul25 & $\cdots$ - C A T T T & C A A G C A T C G T & A T C T T A T C & T GCA TC T - C A & GCA T G G T T T C & T G T A A G T C T C \\
\hline Sbul32 & $\cdots$ - C A T T T & C A A G C A & $\mathrm{TTC}$ & T GCA TC T - C A & G T T T C & G TC TC \\
\hline Sbul15 & $\cdots \mathrm{CA}$ & $\mathbf{G ~ T}$ & & T GCA TA T - C A & GC A & TC T C \\
\hline Sbul3 & $\ldots-C \mathrm{CA}$ & C A A GCA TCG T & C C A T C T & TGCATAT - CA & S T T TC & T TC TC \\
\hline Sbul7 & $\cdots-$ C A T T T & C A A GCA T C G T & C C A TC T TA TC & TGCA TAT $-\mathbf{C A}$ & GCATGGTTTC & TGTAAGTCTC \\
\hline Sbul17 & $\cdots$ - C A T T T & C A A G C A T C G T & C C A TC T T A T C & T GCA TC T - C A & GCA T G G T T T C & T G T A A G C C T C \\
\hline SBul10 & $\cdots$ - C A T T T & C A A G C A T C G T & A T C & T GCA TC T - C A & GCA T G G T T T C & A G T C T C \\
\hline Sbul30 & $\cdots \cdots$ C A T T T & C A A G C A T C G T & $\mathrm{TTC}$ & T GCA T C T - C A & G T T T C & G T C T C \\
\hline SBul14 & $\cdots-$ CA T T T & C A A G C A T C G T & CEATCT & T G C A & $\Gamma \mathrm{C}$ & G TC T C \\
\hline SBul21 & $\cdots-$ CA T T T & C A A G C A T C G T & C C A T C T T A T C & TGCA TC T - C A & GCA T G G T T T C & T G T A A G TC T C \\
\hline Sbul4 & $\cdots$ C A T T T & C A A G C A T C G T & C C A TC T T A T C & T GCA TC T - C A & GCA T G G T T T C & T G T A A G T C T C \\
\hline Sbul9 & $\cdots$ - C A T T T & C A A G C A T C G T & C C A TC T T A T C & T GCA TC T - C A & GCA T G G T T T C & T G T A A G T C T C \\
\hline 31 & $\cdots$ C A T T T & C A A GCA TCG T & C С A TC T TA TC & T GCA TC T - C A & GCA T G G T T T C & T G T A A G T C T C \\
\hline SDUio & $\cdots C$ & C A A G C A & T C & T G C A & G T T T C & TC TC \\
\hline Sbul.CR & $\cdots-$ CA T T T & C A A G C A T C G T & C C A TC T TA T C & T GCA TC T - C A & GCA T G G T T T C & T G TAAGTC TC \\
\hline
\end{tabular}




\begin{tabular}{|c|c|c|c|c|c|c|}
\hline$u l 18$ & T C C A C & $\ldots$ & 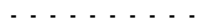 & $-x_{-1}$ & & \\
\hline ul2 & T T C C A C T G T & T G A C G C G A T A & I G G A G A G T G G & T C G A C A T T G C & A A A A G A T G C A & A T A G A A G G C G \\
\hline oul23 & T T C C A C T G T & GAC TCGA T A & G G A G A G T G G & TC GACA T T GC & A A A G G A C G C A & I TAGAAGGG \\
\hline Sbul.CR1 & T T C C AC T G T & TGACGCGATA & GGAGAG T G G & ГCGACA T T GC & A A A GATGCA & TAGA A G GC G \\
\hline Sbul27 & A TCAAC $\cdots$ & $\cdots$ A A T & A A A TCT TC & T TGAA T TCAC & A C T T T G G TA & A G - - \\
\hline Sbul12 & A TCAAC $\cdots$ & $\cdots$ A A T & CAAATCT TC & TTGAATTTAC & A ACTTTGGTA & A TGAAG - - \\
\hline oul13 & $C-\cdots$ & $\cdots-A$ & I TC T TC & Г T GAA T T T A C & A A C T T T G G TA & G - - \\
\hline oul24 & $\Gamma-\cdots$ & $\cdots$ - A A T C & A T C T T C & T - & - TGG T A & I G - - \\
\hline oul28 & T C A A C - - & $\cdots-\ldots$ A A TC & A A A T T T T T & T TGAATT TAC & AАTTTTGGTA & A T GAAG - - \\
\hline Sbul29 & TCAAC $\cdots$ & $\cdots$ A A TC & A A A TC T TC & TTGAATTTAC & ACTTTGGTA & A T G \\
\hline SBul11 & A TC A AC - - & $\cdots$ - A A T & A A TC T TC & TTGAATTTAC & ACTTTGGTA & A A TGAAG - - \\
\hline SBul5 & $\cdots$ & $\cdots-\cdot \mathbf{A}$ & T C & $\mathrm{AC}$ & G T A & G - - \\
\hline ul22 & $-\cdots$ & $\cdots$ A A T & I TCTTC & A C & C T T T G G T A & G - - \\
\hline ul26 & - - - & $\cdots$ - & A TC T TC & T T A C & ACTTTGGTA & A G - - \\
\hline Sbul25 & $-\cdots$ & $\cdots$ - A A T C & A A A T C T T C & T T GA & A C T T T G G T A & A G - - \\
\hline ul32 & $\cdots$ & $\cdots \cdots$ A A T C & I TCTTC & GAA T T T A C & A C T T T & G - - - \\
\hline ul15 & $-\cdots$ & $\cdots$ - A A TC & T C & $\mathrm{T} T$ & A T T T G G TA & G - - \\
\hline 13 & $-\cdots$ & $\cdots$ - & А TC T TC & T T G A & ACTTTGGTA & G - - \\
\hline & $-\cdots$ & $\ldots \ldots$ & TC & & S T A & G - - \\
\hline ul17 & $\cdots$ & $\cdots$ A A TC & TC T TC & A C & G T A & G - - \\
\hline Bul10 & $-\cdots$ & $\cdots$ - & TC T T C & & G G T A & G - - \\
\hline bul30 & $-\cdots$ & 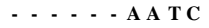 & TC & $\mathbf{T}$ & G T A & G - - - \\
\hline Bul14 & $\cdots$ & $\ldots-\ldots$ & T C & $\mathrm{IC}$ & G T A & G - - \\
\hline 21 & $-\cdots$ & $\cdots \cdots$ & TC & & TA & G - - \\
\hline oul4 & $-\cdots$ & $\cdots \cdots$ & TC & $\mathrm{C}$ & G T A & G - - \\
\hline 9 & $-\cdots$ & $\cdots \cdots A$ & TC & IC & G T A & $G-\cdots$ \\
\hline ul31 & $\cdots$ & $-\cdots-\cdots$ & T C & $\mathrm{CC}$ & G T A & G - - - \\
\hline 16 & $\cdots$ & $\cdots$ A A T C & TC & AC & G T A & G - - \\
\hline & $\cdots$ & $\cdots-A$ & & & T A & \\
\hline & - & $\ldots$ & ( & - & - & - - \\
\hline 12 & A G & $\mathbf{C} \mathbf{T}$ & C T & & G A C & C G \\
\hline 23 & A G & C T & C T & $\mathbf{G}$ & AC & C G \\
\hline & A G & C Y & & & A C & C G \\
\hline ul27 & $\cdots-\cdots G$ & T G C G & A & $\mathrm{T}$ & T T A T & A G \\
\hline ul12 & $\cdots-\cdots G$ & T G T A & & & $\mathbf{A} \mathbf{T}$ & \\
\hline ul13 & $\cdots-G$ G & T T G T A & & & A T & \\
\hline 4 & $\cdots-G$ G & $\Gamma-$ & A A & $\mathrm{T}$ & S A T & A G \\
\hline & $\cdots-\cdots G$ & C G & & & A T & A G \\
\hline ul29 & $\cdots-\cdots G$ & Г T GCG & A & & A $\mathbf{T}$ & $\mathbf{T}$ \\
\hline SBul11 & $-\mathbf{G}$ & G & & & $\mathbf{A} \mathbf{T}$ & \\
\hline 5 & $--_{-1}$ & C G & & & A T & \\
\hline$u l 22$ & $\cdots-\cdots G$ & C G & A & $\mathrm{T}$ & A T & $\mathbf{A} \mathbf{G}$ \\
\hline 0 & $\cdots-\cdots G$ & C G & & & A $\mathbf{T}$ & \\
\hline oul25 & $\cdots-\cdots G$ & C G & & & A T & A G \\
\hline ul32 & $\cdots-\cdots G$ & & & & A T & \\
\hline & & & & & A $\mathbf{T}$ & \\
\hline & $\cdots-\cdots G$ & C G & A & $\mathbf{T}$ & A $T$ & A G \\
\hline 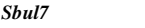 & $\cdots-\cdots G$ & C G & & & A T & \\
\hline oul17 & $\cdots-\cdots G$ & T G C G & A A & $\mathrm{T}$ & T G A T & A G \\
\hline & $\cdots-G$ G & & & & A T & \\
\hline & & G C G & & & & \\
\hline & $\cdots$ & T T G G & & & & \\
\hline SBul21 & $-\ldots-\cdots$ & T T G G & & & T GA T T & 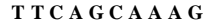 \\
\hline Sbu & $\cdots-G G$ & A C T T A T T G C G & G G C A A A C A A A & C T GA T G T T A T & Г A T A T T G A T & T T C A G C A A A G \\
\hline Sbu & $\cdots-G$ & A T T G C G & & $\mathrm{T}$ & A T A T T G A T & T T C \\
\hline & & T T A T T G C G & & & & \\
\hline & - n & & & & & \\
\hline & $-5-5$ & H $1 \mathrm{~A}$ & & & A 1 & 列 \\
\hline
\end{tabular}




\begin{tabular}{|c|c|c|c|c|c|c|}
\hline Sbul18 & C - GTTTAACC & CGGCGAATTG & GGGGCG - - - & GATTATGAGA & GCCCTTTTCA & ACATGG $\cdots$ T \\
\hline Sbul2 & - G T T TAAC T & CGGCGGATTG & GGGGCG - - - & GATTATGAGA & GCCCTTTTCA & ACATGG- - C T \\
\hline Sbul23 & C-GTTTAACT & CGGCGAATTG & GGGGTG $\ldots$ & GATTATGAGA & GCCCTTTTCA & ACATGG- C \\
\hline Sbul.CR1A.Con & C- G T T TAACT & CGGCGAATTG & GGGGCG - - - & GATTATGAGA & GCC C T T T TCA & ACATGG- - C T \\
\hline Sbul27 & C-ATTTGATA & A A A TAAATCA & TAACTTACTC & G T TCCTAAG - & - - C T T TA TCA & ACTTGGTTTT \\
\hline Sbul12 & - A T T T GA T A & A G T A A A T C A & TAACTTACTC & G T TCA TAAG - & - - C T T TA TCA & A C T T T G \\
\hline Sbul13 & - АTTTGATA & GAGTAAATCA & TAACTTACTC & GTTCATAAG - & - - С T T TA TCA & ACTTTGTTTT \\
\hline Sbul24 & C-ATATGATA & AAGTAAATCA & TAACTTACTC & GTTCATAAG - & - - С T T TA TCA & ACTTGGTTTT \\
\hline Sbul28 & C - A T TTGATA & A AGTAAACCA & TAACTTACTC & GTTCTTAAG - & - - A T TTA T TCA & A- TTGGTTTT \\
\hline Sbul29 & CKA T T TGATA & A A G TA AMC C A & TAACTTAYTT & GTTCATAAG - & - - C T T TA TCA & ACTTGGTTTT \\
\hline SBul11 & C - A TTTGATA & C C A & A C T T & G T TCA TAAG - & - - C T T TA TCA & A C T T G G \\
\hline SBul5 & C-ATTTGATA & A A G TAAACCA & TAACTTACT T & GTTCATAAG - & - - C T T TA TCA & ACTTGGTTTT \\
\hline Sbul22 & C - A TTTGATA & A AGTAAACCA & TAACTTACT T & GTTCATAAG - & - - C T T TA TCA & ACTTGGTTTT \\
\hline Sbul26 & C-ATTTGATA & A A G TAAACCA & TAACT TAC TC & G T TCA TAAG - & - - C T T TATCA & ACTTGGTTTT \\
\hline Sbul25 & C-ATTTGATA & A A G TAAACCA & TAACT TAC TC & G T TCA TAAG - & - - C T T TATCA & ACTTGGTTTT \\
\hline Sbul32 & C - A T T TGATA & A A G TAAACCA & TAACTTACTC & G T TCATAAG - & - - C T T TACCA & AC T T G G \\
\hline Sbul15 & - ATTTGATA & A AGTAAACCA & TAACTTACTC & GTTCATAAG - & - - C T T TA TCA & AC T T G G \\
\hline Sbul3 & - АTTTGATA & A A GTAAACCA & TAACTTACTC & GTTCATAAG - & - - С T T TA TCA & ACTTGGTTTT \\
\hline Sbul7 & C - A T TTGATA & A A G TAAACCA & TAACT TAC TC & G T TCA TAAG - & - - C T T TATCA & ACTTGGTTTT \\
\hline Sbul17 & C-ATTTGATA & A A G TAAACCA & TAACT TAC TC & G T TCA TAAG - & - - A T TTATCA & ACTTGGTTTT \\
\hline SBul10 & C- ATTTGATA & A A G T A & TAAC T T & G T TCA T & - - С T T TA TCA & AC T T G G \\
\hline Sbul30 & - ATTTGATA & A AGTAAACCA & TAACTTACTC & GTTCATAAG - & - - C T T TA TCA & ACTTGGTTTT \\
\hline SBul14 & C - A T TTGATA & A A G T A A & T A A C T T T & G - & TC A & AC T T \\
\hline SBul21 & C - A T T TGATA & A A G T A A A C C A & TA AC T TAC TC & G T TCA TAAG - & - - C T T TA TCA & ACTTGGTTTT \\
\hline Sbul4 & C - A T TTGATA & A A G TAAACCA & T A A C T T T & G T T C A T T & - - C T T TATCA & A C T T G G \\
\hline Sbul9 & $\mathbf{C}-\mathbf{A} \mathbf{T}$ T T G & ACC A & A C T T & G T TCATAAG - & - - C T TTATCA & AC T T G G \\
\hline Sbul31 & - ATTTGATA & A A GTAAACCA & TAACTTACTC & GTTCATAAG - & - - C T T TA TCA & ACTTGGTTTT \\
\hline SBul6 & T T T G A T A & A A G T A & TA A C T T & G T T C & - - C T T TA TCA & AC T T G G \\
\hline Sbul.CR1B.Con & C - A T T T GA TA & A A G T A A A C C A & TA AC T T A C T C & G T T C A T & T C A & ACTTGGTTTT \\
\hline Sbul18 & & G G A & $T$ & $\Gamma \mathrm{C}$ & I C A G G A A & 001 \\
\hline Sbul2 & CGCCTCCA T & ACTTAATGGA & T TC TGAA T & GATTACTTTC & AGGACAGGAA & GG T A \\
\hline Sbul23 & C CGCCTCCA T & ACTTAATGGA & TTCTGAA T & GATTACTTTC & A GGACAGGA A & GGTAATATAC \\
\hline Sbul.CR1 & ACGCCTCCA T & ACTTAATGGA & GATTCTGAAT & GA T T A C T T T C & A G GACAGGA A & G G T A A T \\
\hline Sbul27 & A G T T C A T & C T G & $\mathbf{A} T \mathbf{T}-\cdots \mathbf{A}$ & A C G T A T T & A C A & $\mathbf{G C}-\mathbf{A}$ \\
\hline Sbul12 & C A T & A C T G & T - A A A & T C G T A & A G A G G A A & GC - A \\
\hline Sbul13 & GTTCATCAT & TACTAAACTG & GA T T & TCGTATTTAG & TA A A G A G G A A & GC-AGTGCGT \\
\hline Sbul24 & GCTCATCAT & AC T G & GA T T $T-$ A A A & TCGTATTTAG & TAA ACAGA A & GC-AGTGCGT \\
\hline Sbul28 & A G T T GG TCA T & TAC TAAA T T G & GA T T $\cdots$ A A G & TCGTATTTAG & $\mathbf{T}-\mathbf{A} \mathbf{A} \mathbf{C} \mathbf{A} \mathbf{G A} \mathbf{A} \mathbf{A}$ & GC-AGTGCGT \\
\hline Sbul29 & AGTTCGTCAT & ACT T & GA T T T - A A G & TCGTATTTAG & A A A & GC-AGTGCGT \\
\hline SBul11 & A G T T C G T & C T G & $\Gamma \mathbf{T} \cdots \mathbf{A} \mathbf{A} \mathbf{G}$ & G TA T & I C A G A A A & GC - A \\
\hline SBul5 & AGTTCGTCAT & TACTAAACTG & GA T T T - A A G & TCGTATTTAG & TAAACAGAA & GC-AGTGCGT \\
\hline Sbul22 & AGTTCGTCAT & TAC TAAAC TG & GA T T & TCGTATTTAG & TAAACAGAA & GC-AGTGCGT \\
\hline Sbul26 & A G T TCGTCAT & T A C T A A A C T G & GA T T $\cdots$ A A G & TCGTA T T TA G & T A A A C A G A A A & GC - A G T GC G T \\
\hline Sbul25 & A G T TCG TCA T & AC T G & GA T T $\cdots$ A A G & TCGTATTTAG & TA A A C A G A A A & GC-AGTGCGT \\
\hline Sbul32 & A G T T C G T & C T G & A G & TCG TA T & C A G A A A & GC-AGTC \\
\hline Sbul15 & AGTTCGTCAT & TACTAAAC TG & GA T T T - A A G & TCGTATTTAG & TAAACAGAA & GC-AGTGCGT \\
\hline Sbul3 & AGTTCGTCAT & T A C T A & $\mathbf{A} \mathbf{T} \mathbf{T}--$ A A G & TCGTATTTAG & A C A G A A A & GC-AGTGCGT \\
\hline Sbul7 & A G T TCGTCAT & TACTAAAC T G & GATT & TCGTATTTAG & TA A A C A G A A A & GC - A G T GCG T \\
\hline Sbul17 & A G T T C G T & AC T G & GA T T $\cdots$ A A G & TCGTATTTAG & TA A A C A G A A A & GC-AGTGCGT \\
\hline SBul10 & G T T C G T C A T & TAC T A A A C T G & GATT & TCGTA T T TAG & TAAACAGA A & GC-AGTGTGT \\
\hline Sbul30 & GTTCGTCAT & TAC TAAAC TG & GA T T T - A A G & TCGTATTTAG & TAAACAGAA & GC-AGTGCGT \\
\hline SBul14 & AGTTCGTCAT & TACTAAAC TG & GA T T T - A A G & TCGTATTTAG & TAAACAGAA & GC-AGTGTGT \\
\hline SBul21 & AGTTCGTCAT & TAC TAAAC T G & GA T T $\ldots$ A A G & T C G T A T T T A G & T A A A C A G A A A & GC - A G T G T T \\
\hline Sbul4 & A G T T C G TCA T & TAC T A A A C T G & GA T T $\cdots$ A A G & T C G T A T T T A G & T A A A C A G A A A & GC - A G T G C G T \\
\hline Sbul9 & G T TCG TCA T & T A C TA A A C T G & GA T T $\cdots$ A A G & T C G T A T T TA G & TAAACAGA A & GC-AGTGCGT \\
\hline Sbul31 & G T T C G T C A T & T A C T A & G A T T $\cdots$ & T C G T A T T T A G & I C A G A A A & GC - A G T G C G T \\
\hline SBul6 & AGTTCGTCAT & TACTAAAC TG & GA T T T - - & TCGTATTTAG & TAAACAGAA & GC-AGTGCGT \\
\hline Sbul.CR1B.Co & AGTTCGTCAT & TAC TAAAC TG & GA T T T - A A G & TCGTATTTAG & TA A A C A G A A A & A G T G C G T \\
\hline
\end{tabular}




\begin{tabular}{|c|c|c|c|c|c|}
\hline Sbul18 & CAAATGGATG & A A T GGAGAAA & A A G A & $\cdots \cdots$ & \\
\hline Sbul2 & A A A C G G A T G & - A T G GA GAA A & GGA A T A C G T & - A G T G A C G & \\
\hline Sbul23 & AAACGGATG & - A TGGAGAAA & GGAATACGT & - A G T T A C G & \\
\hline bul.CR1A.Con & A A ACGGATG & - A T GGAGAAA & GGARTACGT & - AG T T A C G & C G \\
\hline Sbul27 & A A A T TCAGG & A ACA TGCA A T & A A G T T TA T & TGATGTACTA & \\
\hline Sbul12 & A A A T TCAGG & A ACACGCA A T & GAACTTTAT & TGATGTACTG & $\mathrm{C}$ \\
\hline Sbul13 & T T C A G G & GCA A T & T T T A T & TGA TG TAC TG & \\
\hline bul24 & A G G & A T & T T A A T & TGATGTACTG & \\
\hline bul28 & C - - - & & & $\ldots \ldots$ & \\
\hline Sbul29 & C A G G & A ACACACA A T & C GAACTTAA T & TGA TGTACTG & \\
\hline SBul11 & C A G G & & T T A A T & TGATGTACTG & $\mathrm{C}$ \\
\hline Bul: & I G G & & $T$ & G T A C T G & C \\
\hline bul22 & C A G G & A T $\mathbf{T}$ & T T A A T & TGATGTACTG & C \\
\hline Sbul26 & G G & A $T$ & A $\mathbf{T}$ & TGATGTACTG & C \\
\hline Sbul25 & C A G G & & A T $\mathbf{T}$ & T G A T G T A & 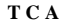 \\
\hline b & G G & & & T G T A C T G & \\
\hline Sbul15 & G G & $T$ & $\mathrm{~A} T$ & T G T A C T G & C \\
\hline Sbul3 & A G G & $\mathrm{T} T$ & A T & TGATGTACTG & C \\
\hline Sbul7 & A G G & A T & A T & TGATGTACTG & $\mathrm{CA}$ \\
\hline Sbul17 & C A G G & A A & T T A A T & T GA T G T A C T G & ГC T \\
\hline Bul10 & I G G & & & TGATGTACTG & C \\
\hline Sbul30 & & & & & \\
\hline SBul14 & & & & T G & C \\
\hline SBul21 & G G & A A & T T A A T & TGATGTACTG & C \\
\hline Sbul4 & C A G G & A A & T T A A T & TGA T G TAC T G & TC \\
\hline Sbul & A G G & & & G T A C T G & C \\
\hline & A G G & & & G T A C T G & \\
\hline & & & & T GA T G T A & \\
\hline Sbul.C & A G G & $\mathrm{T}$ & C GAAC T TAA T & TGA TG TAC T G & \\
\hline
\end{tabular}

Supplemental Figure 5 Sequences obtained from $S$. bullata clones alongside consensus clusters 


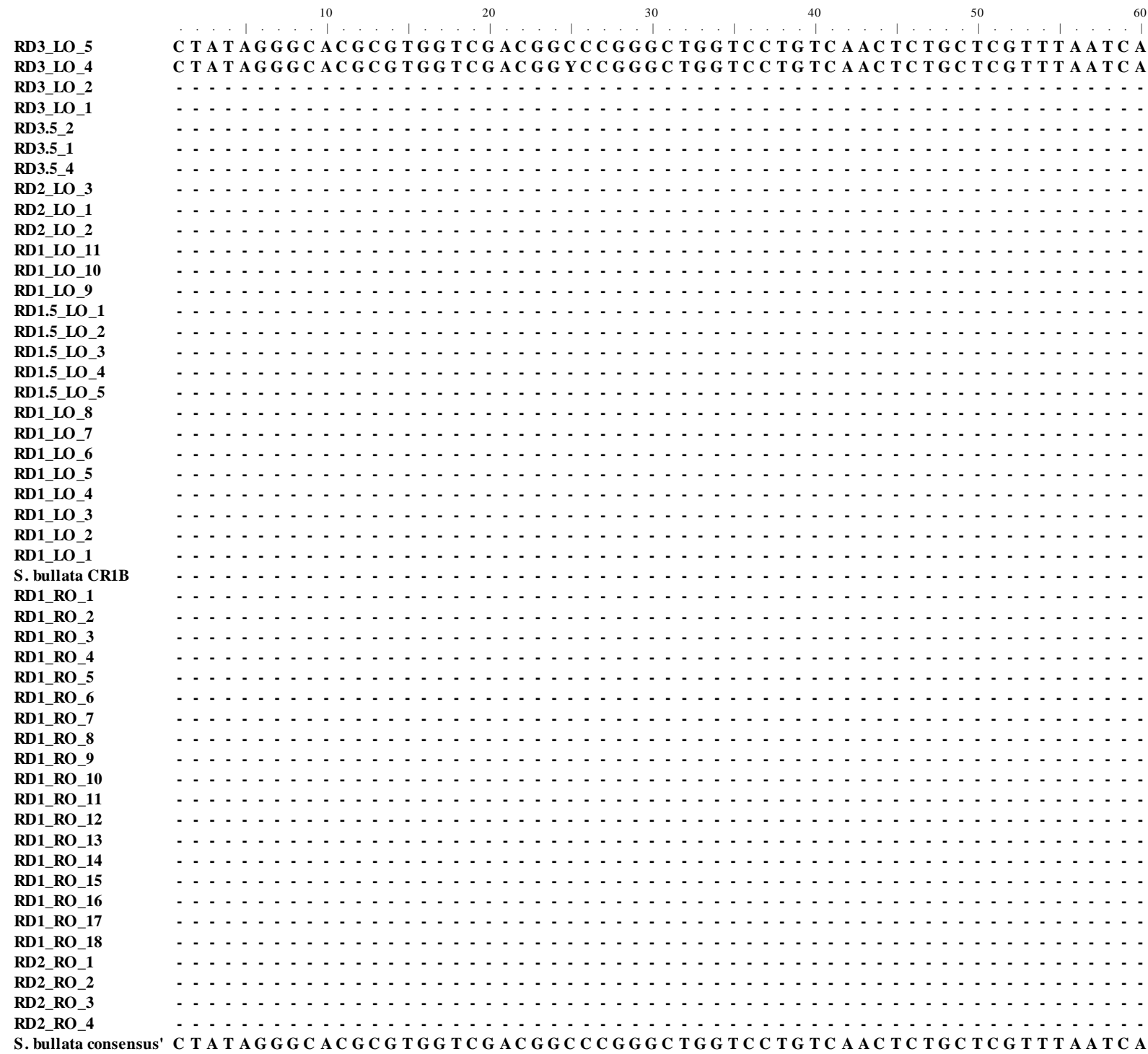




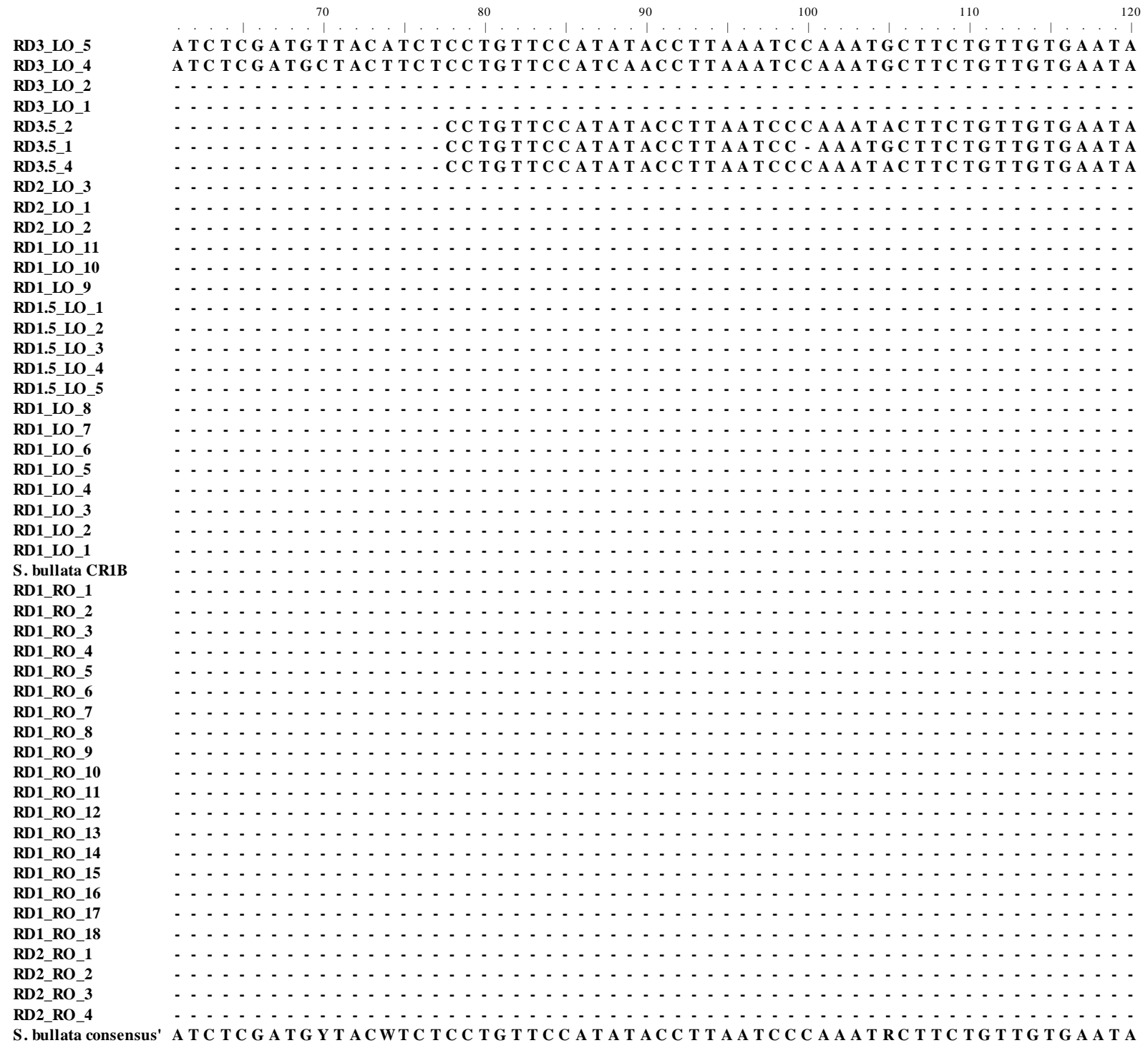




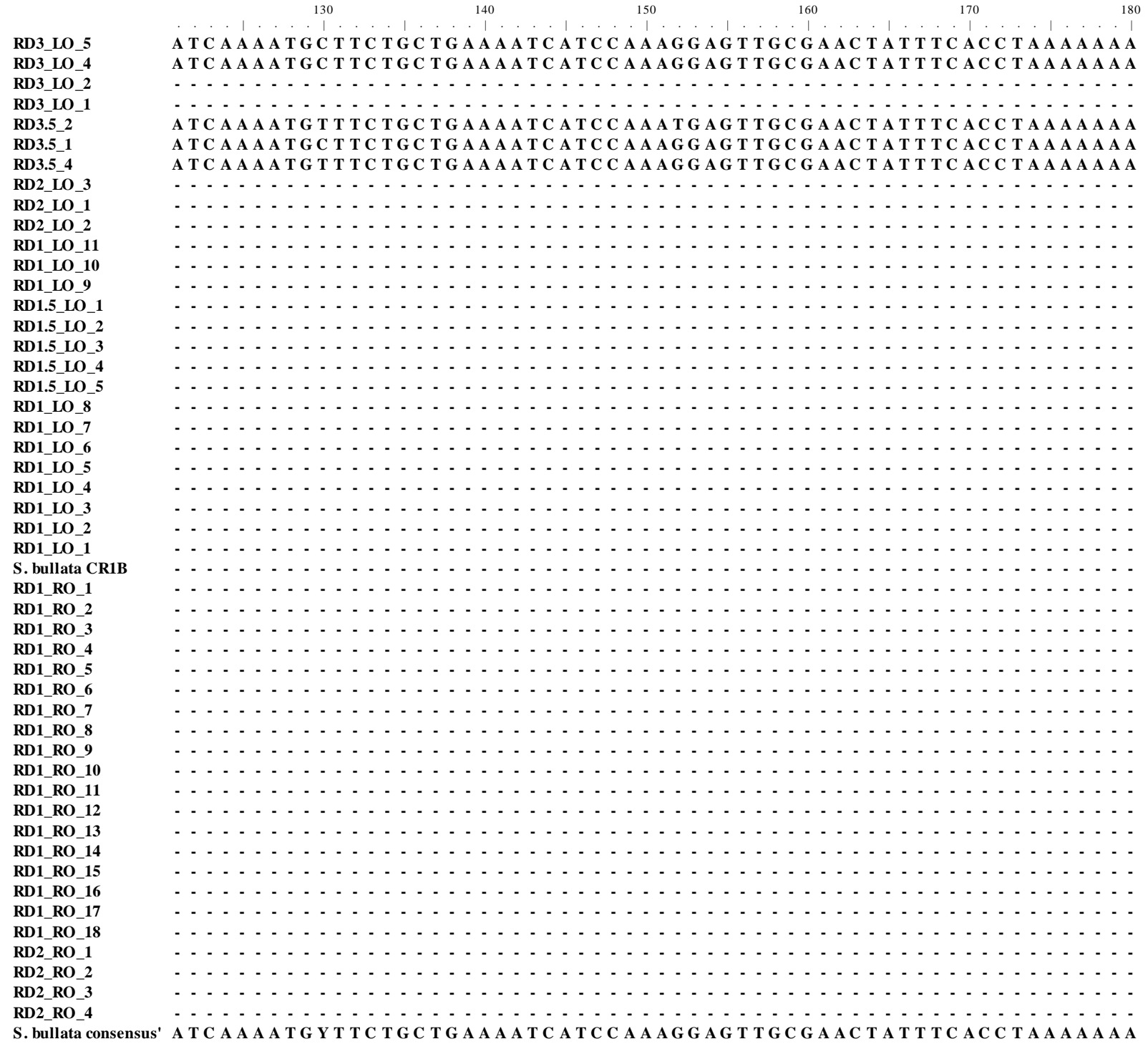




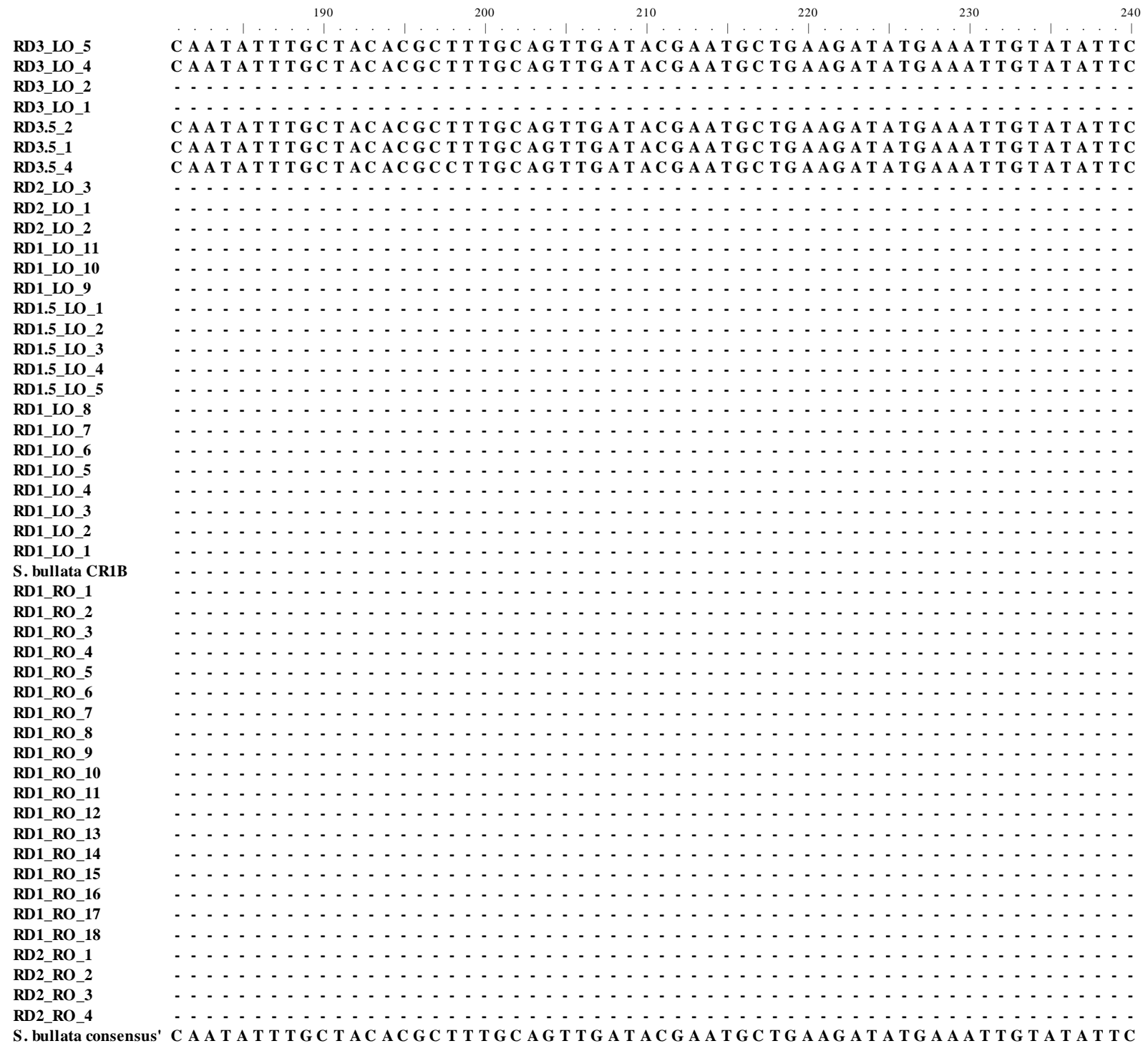




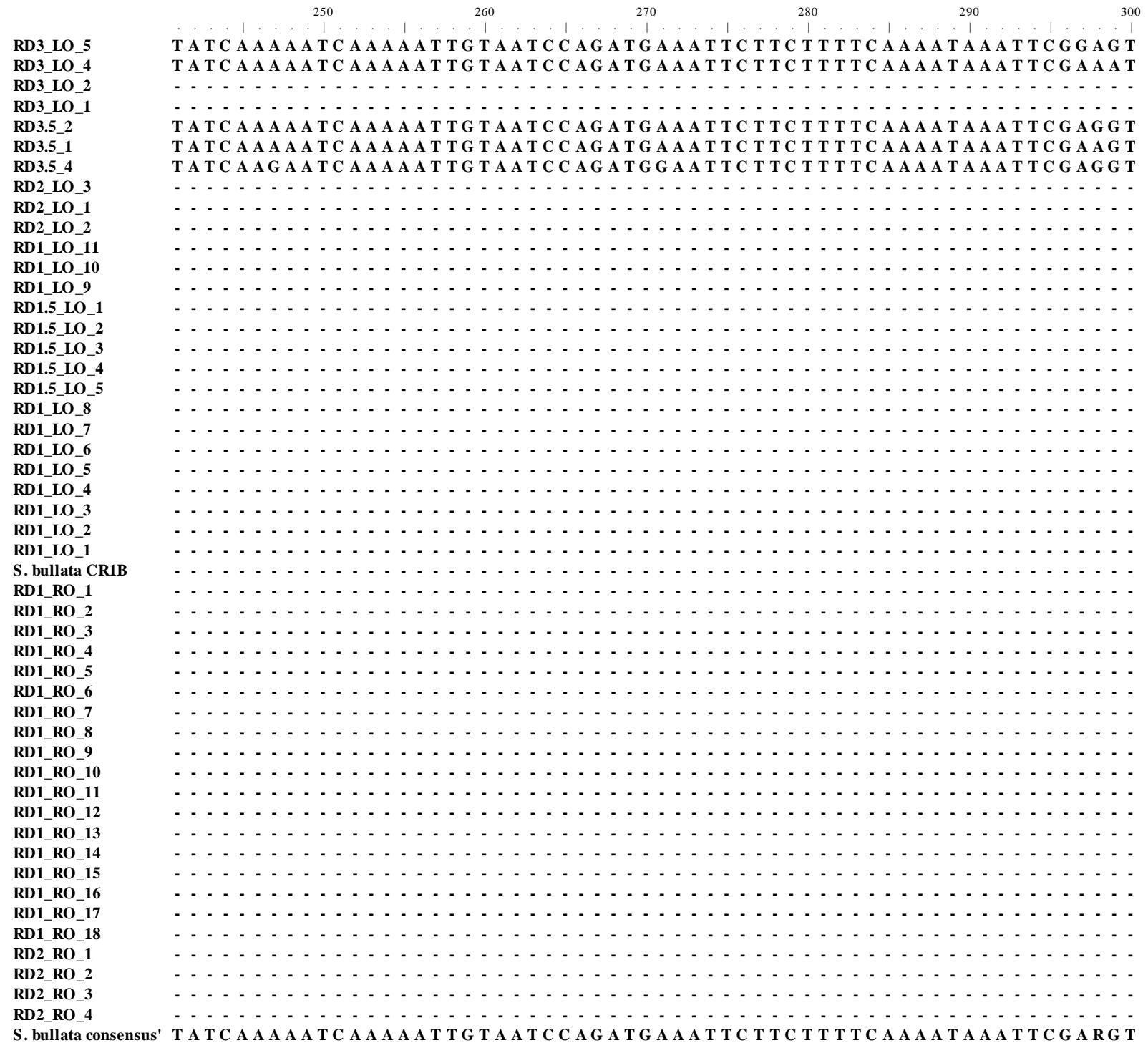




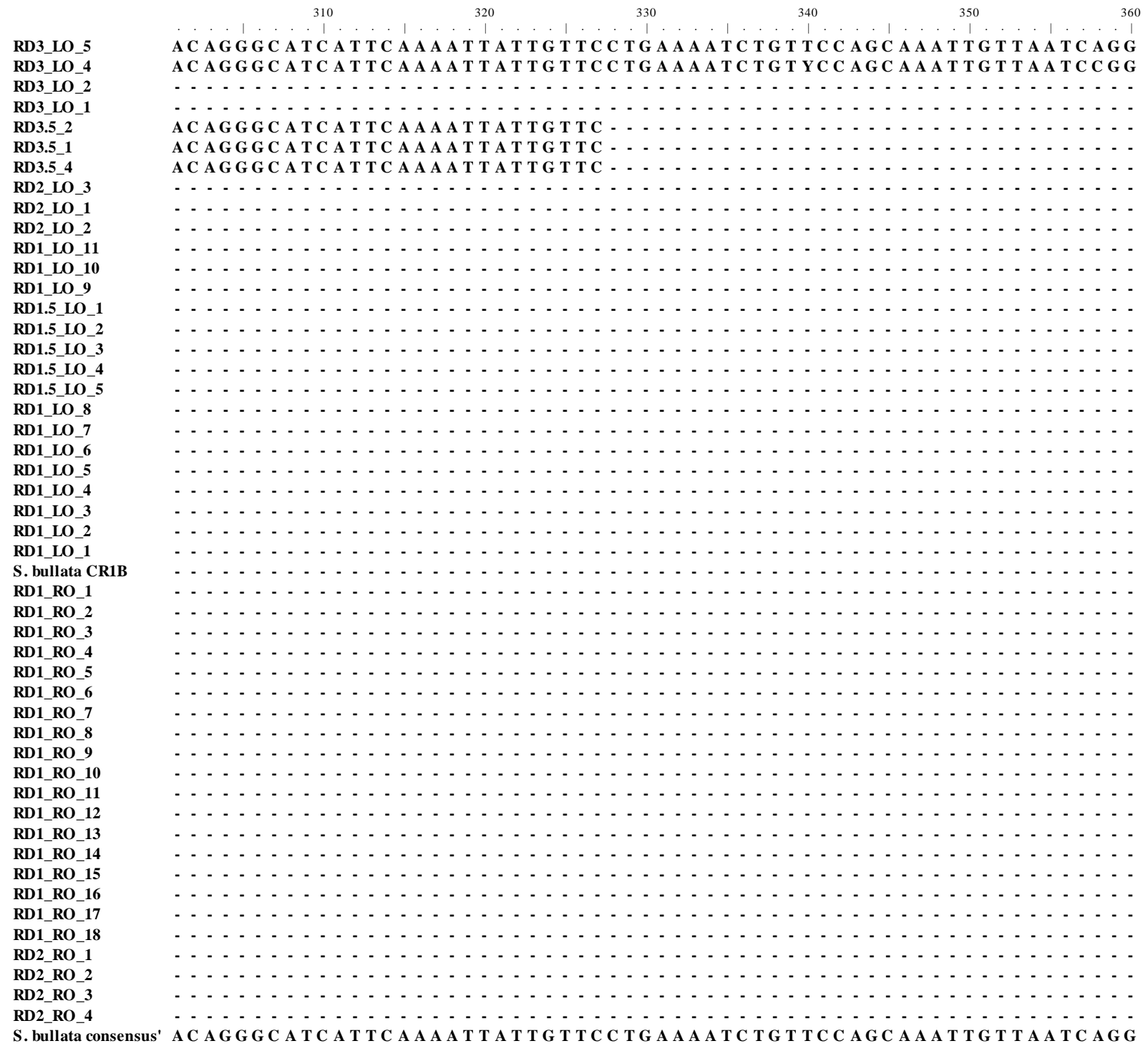




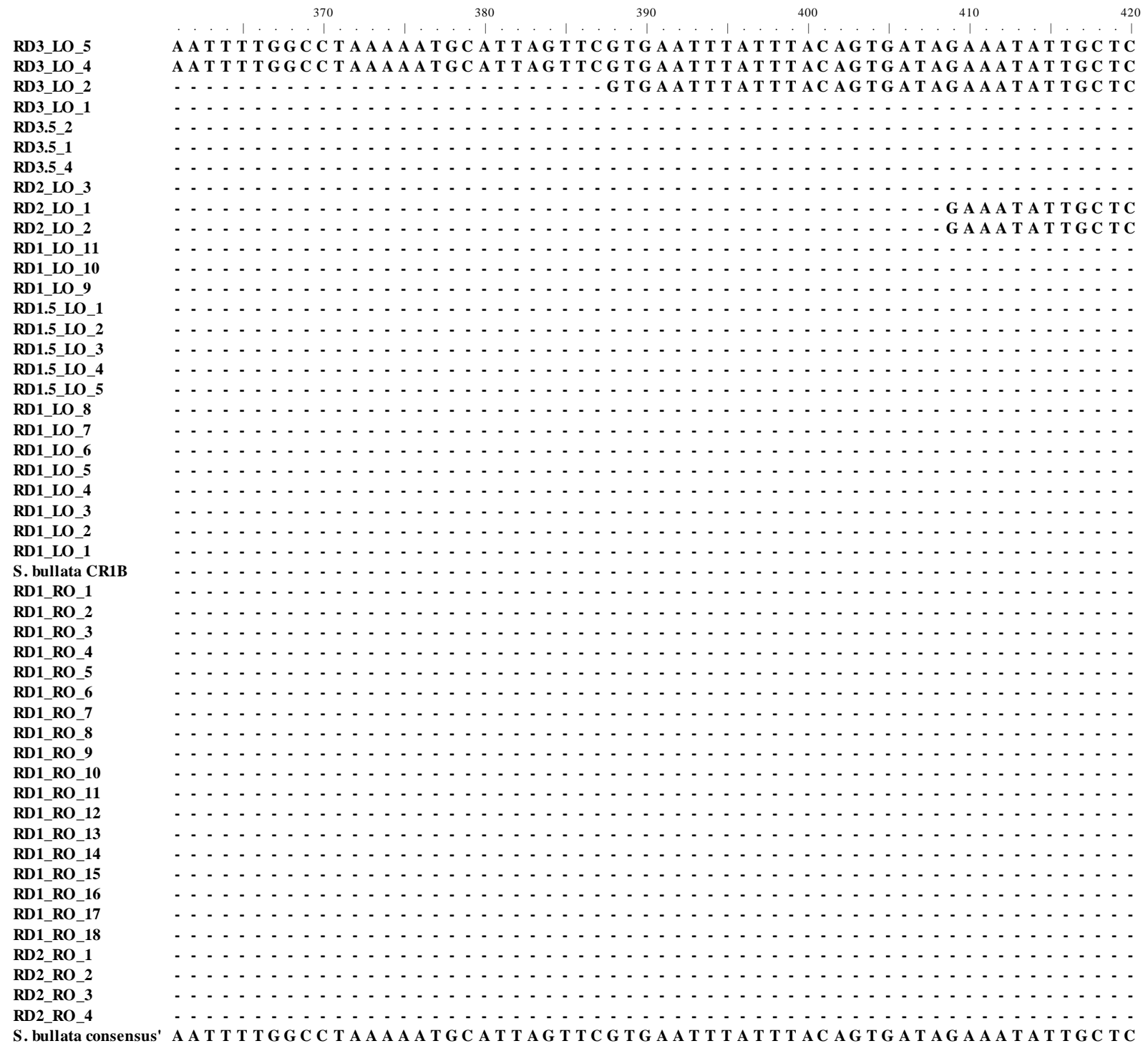




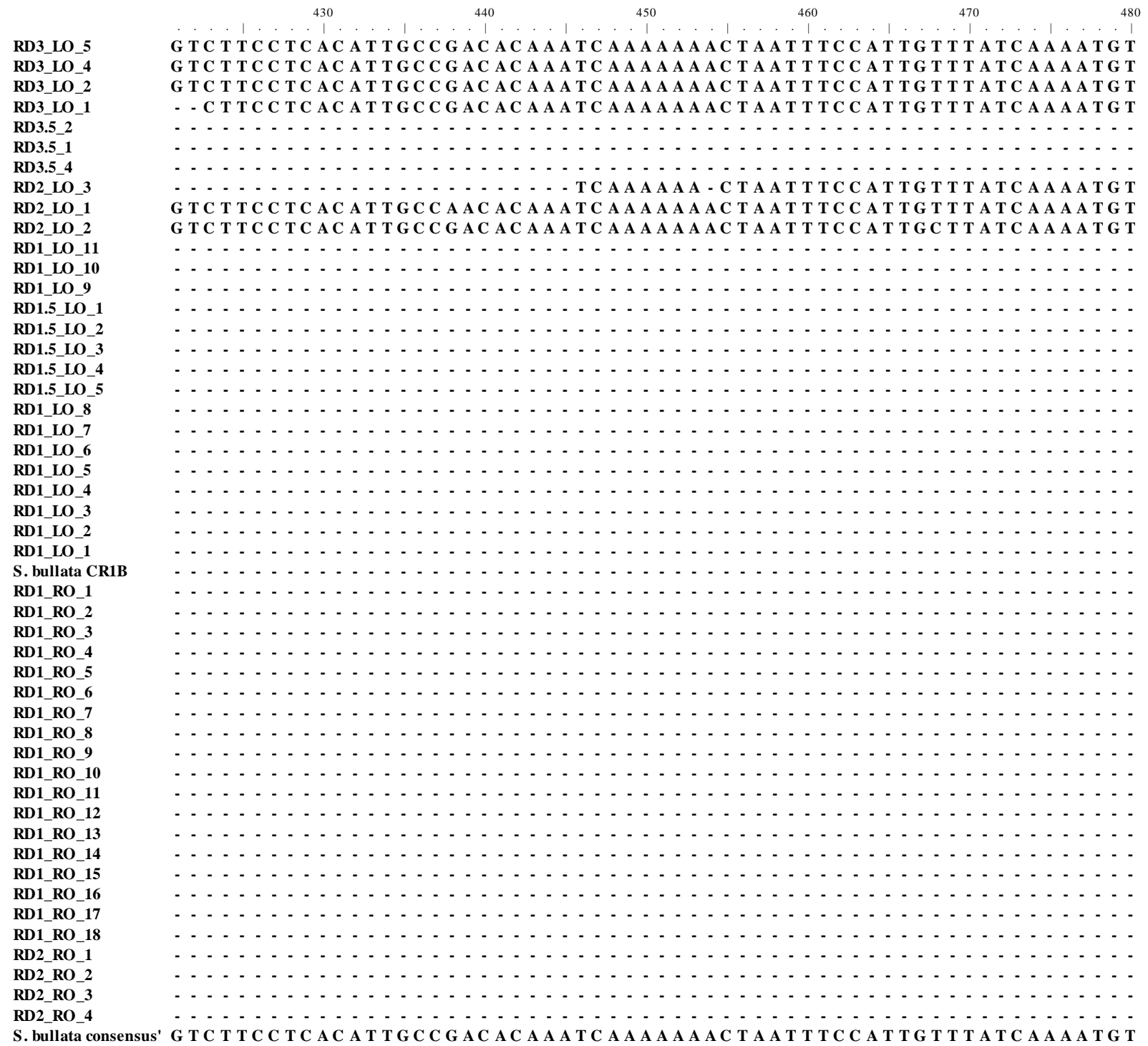




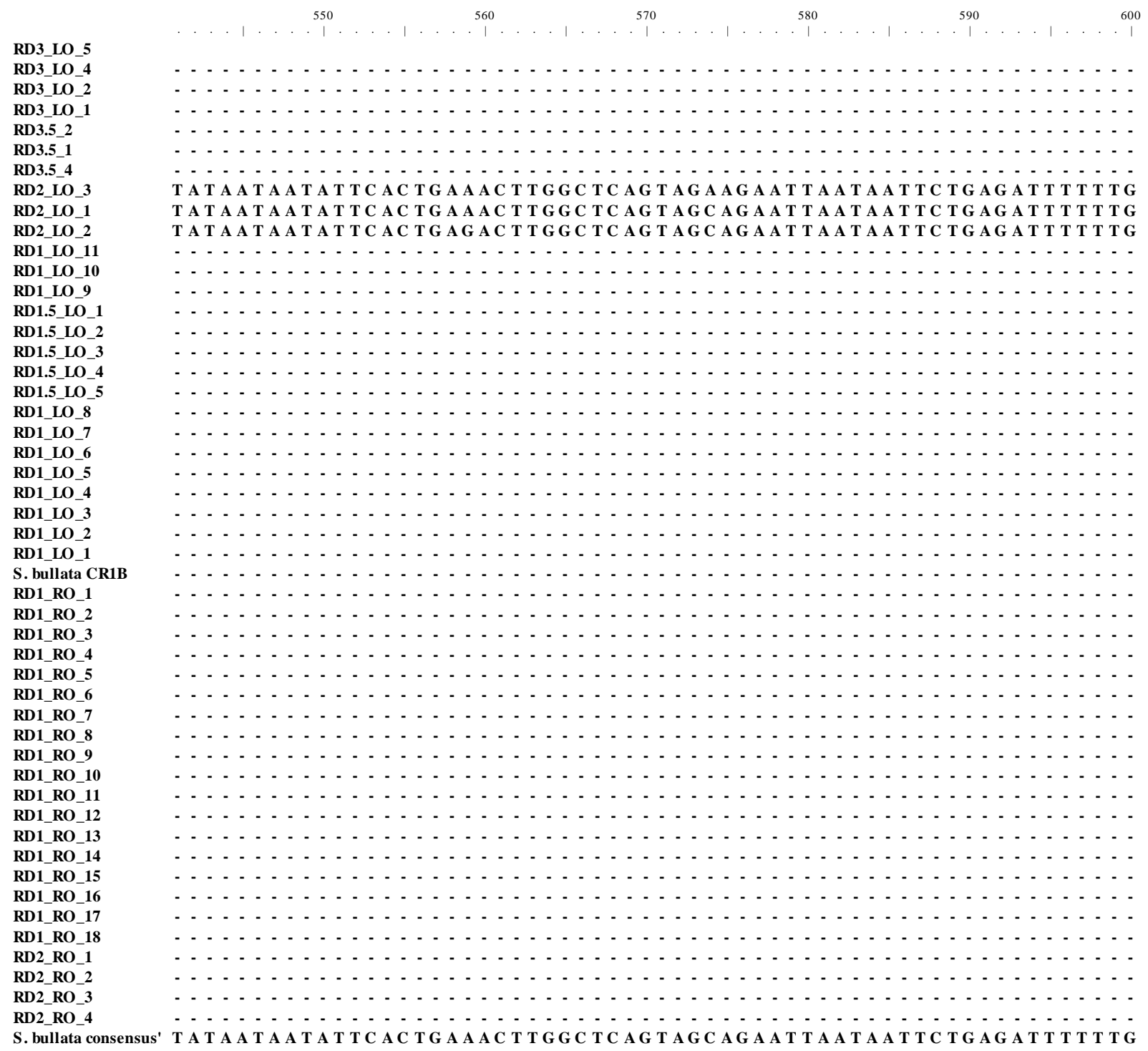




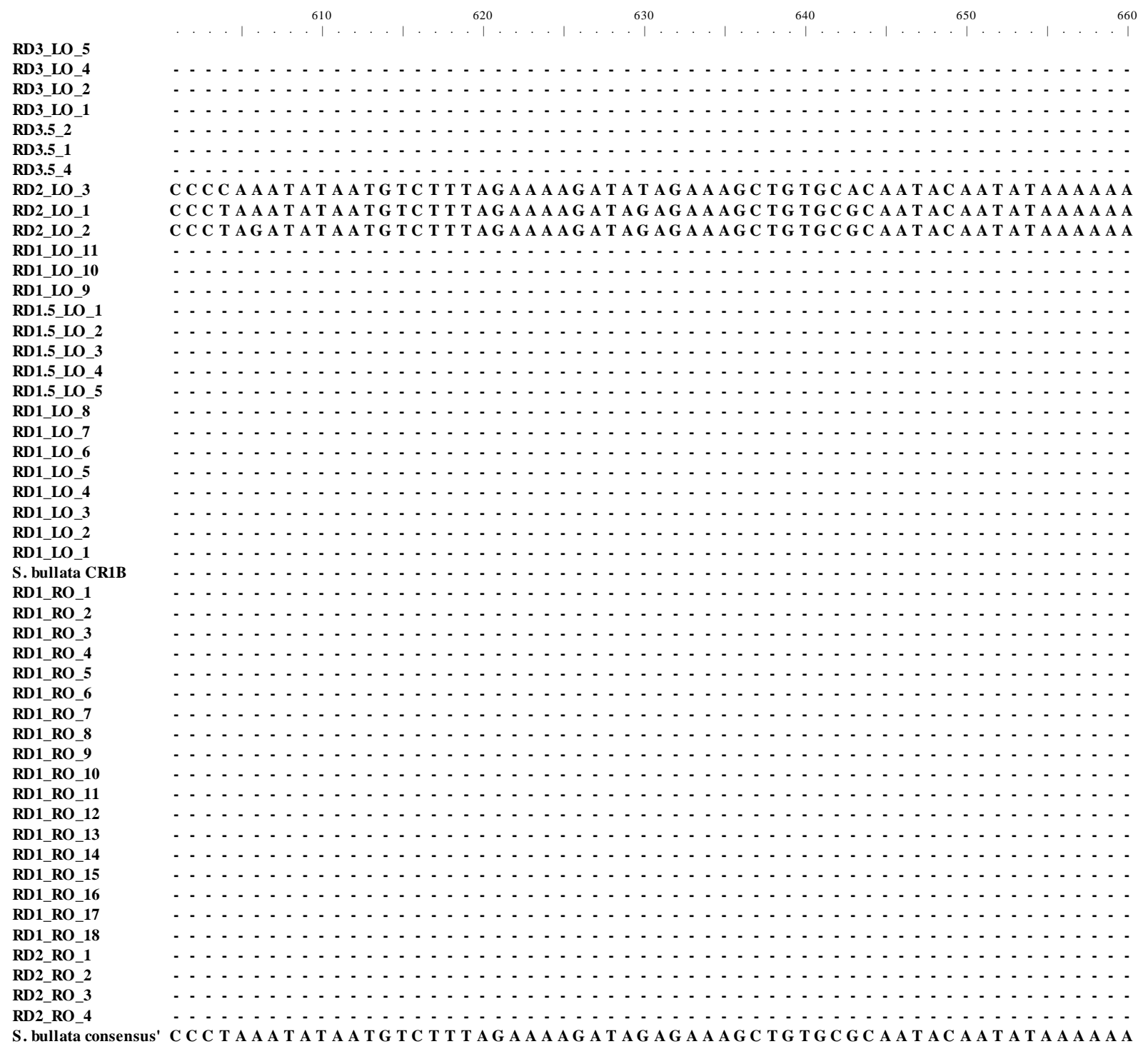




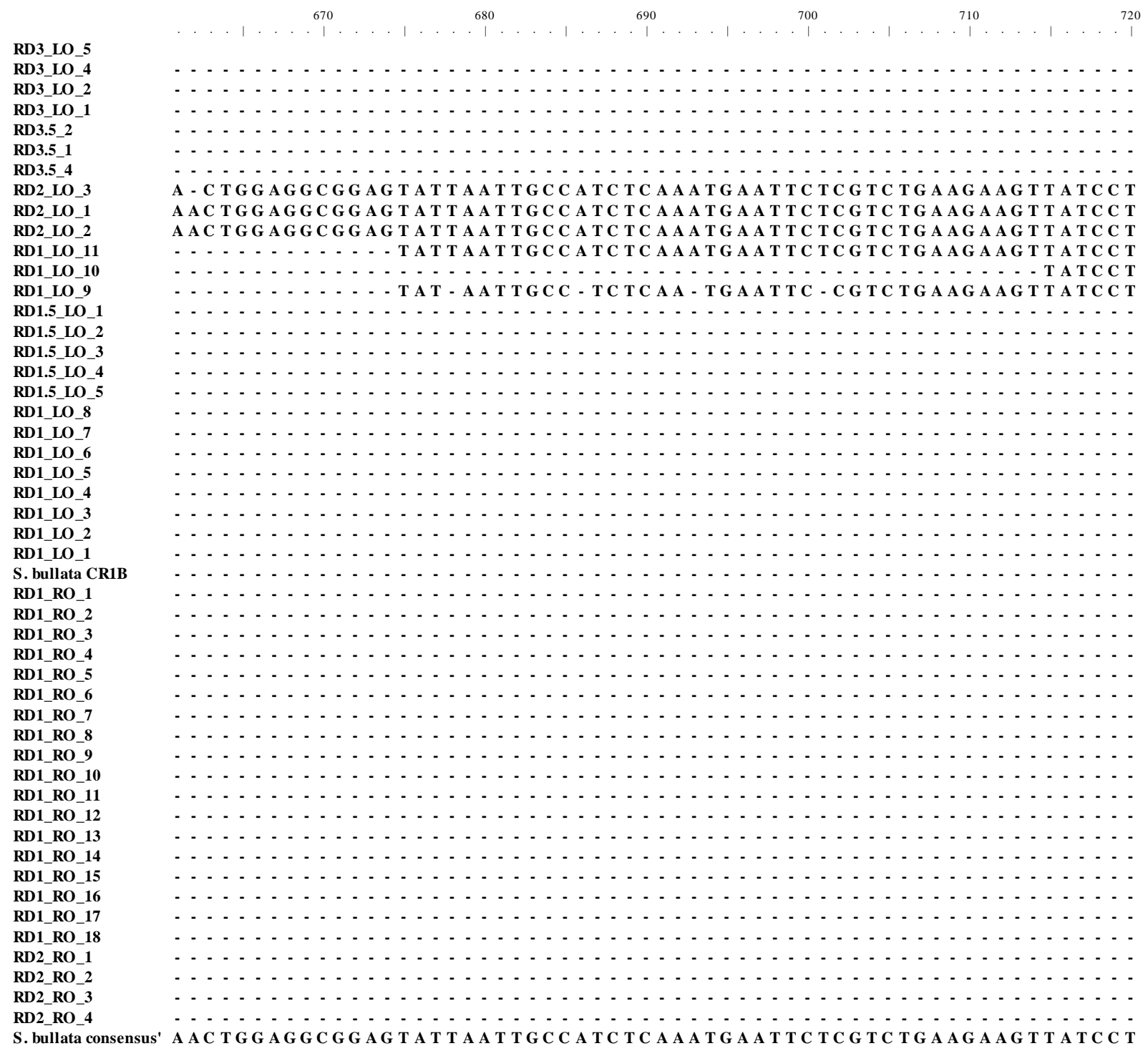




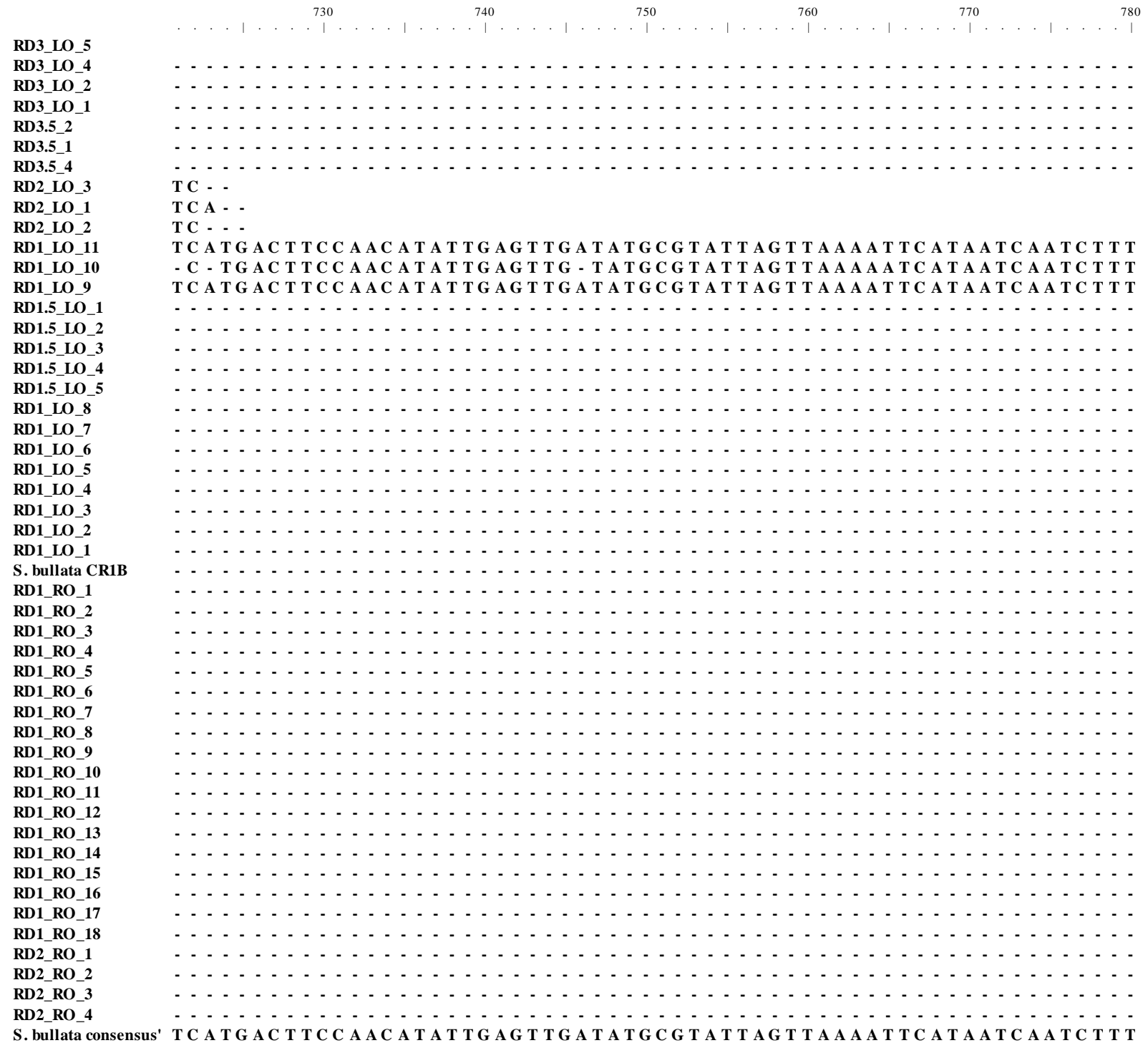




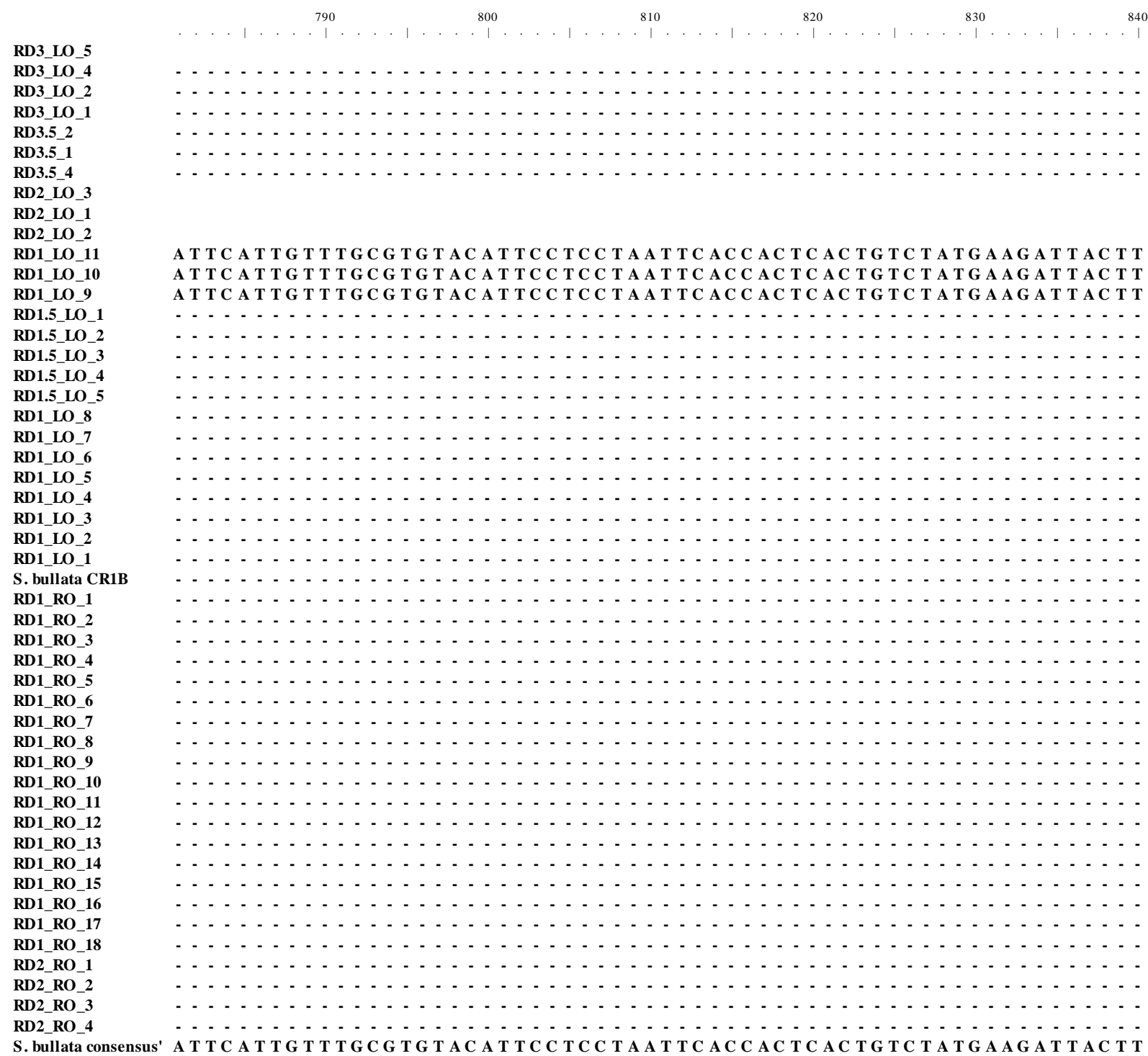




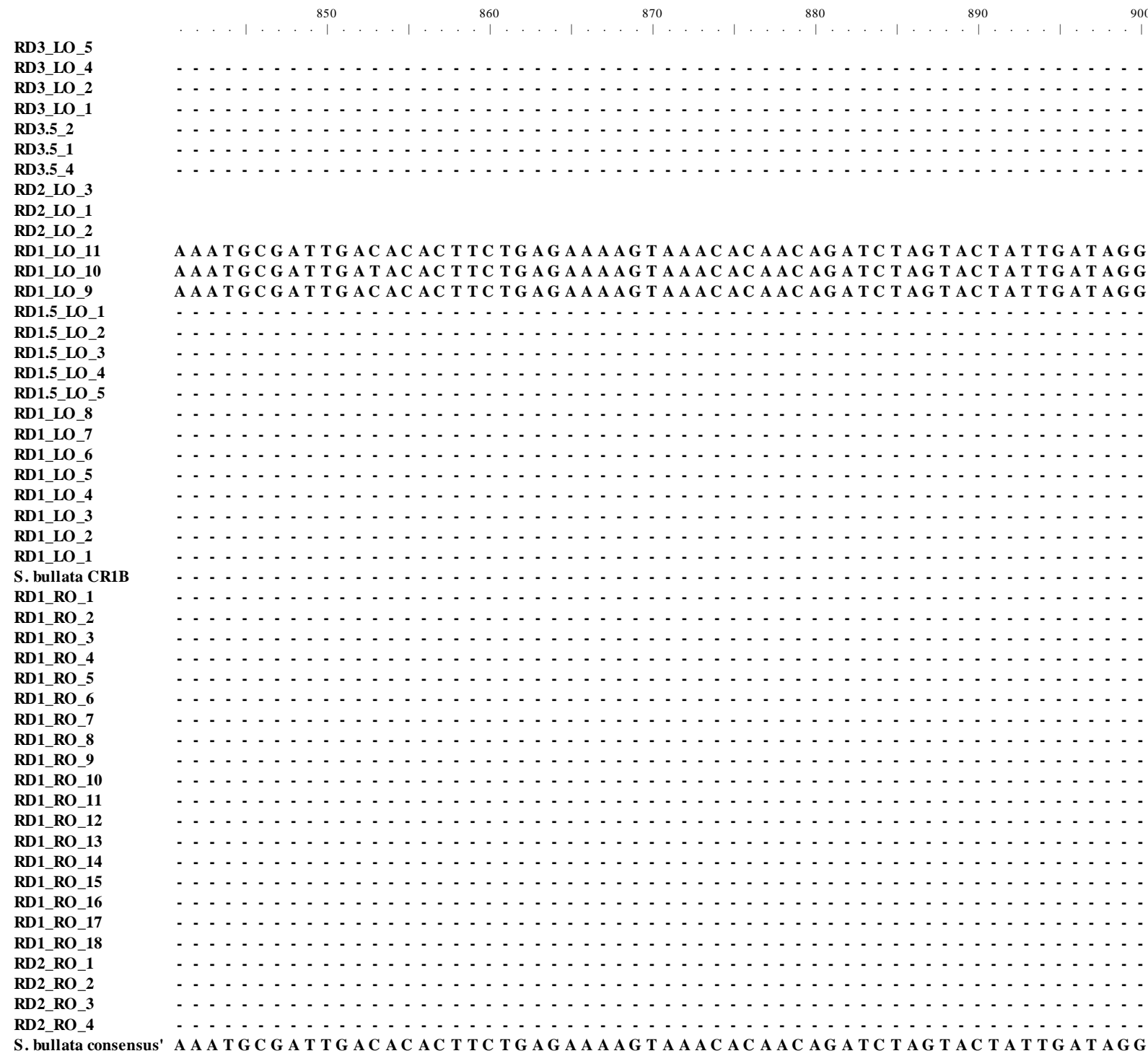




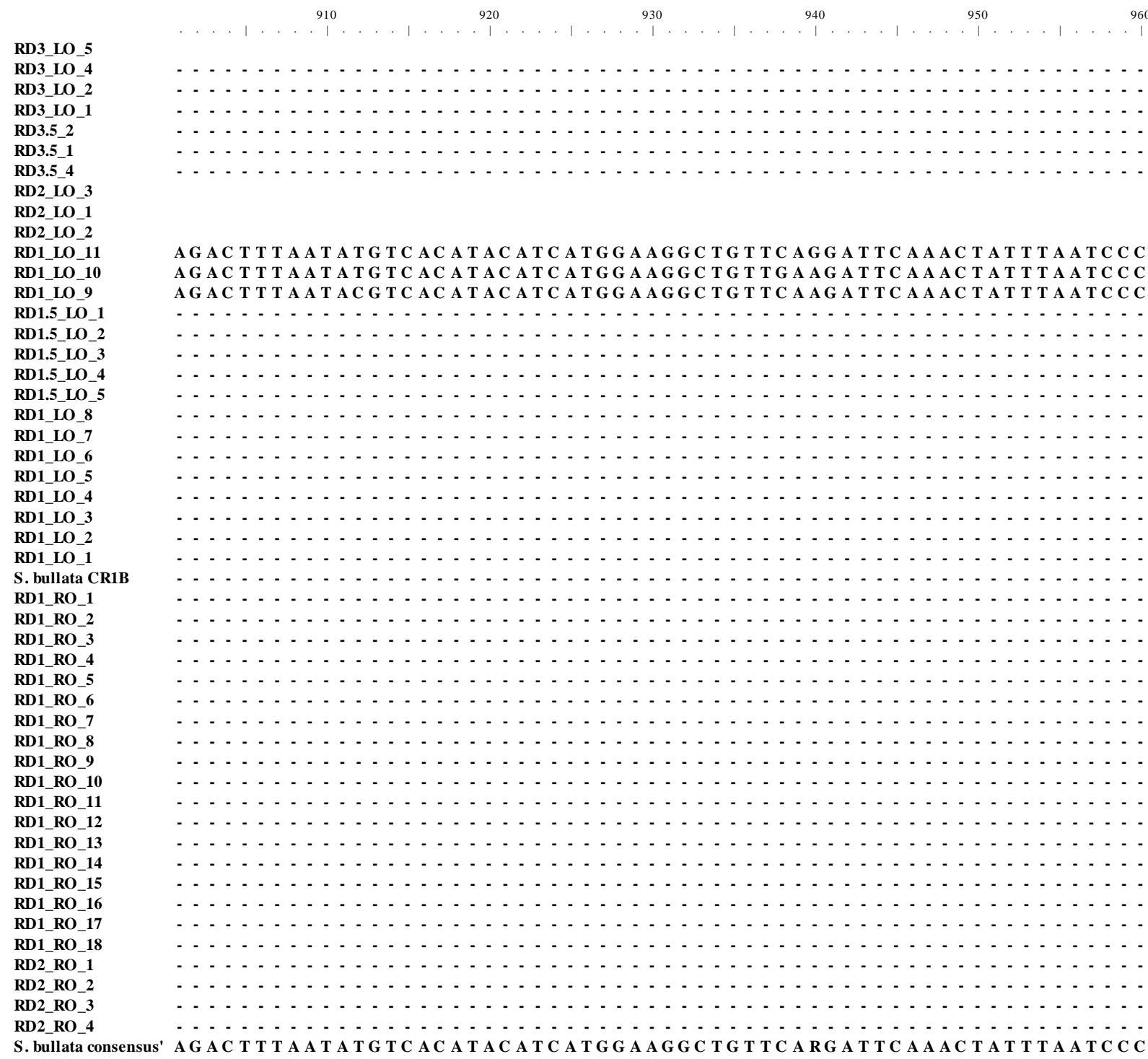




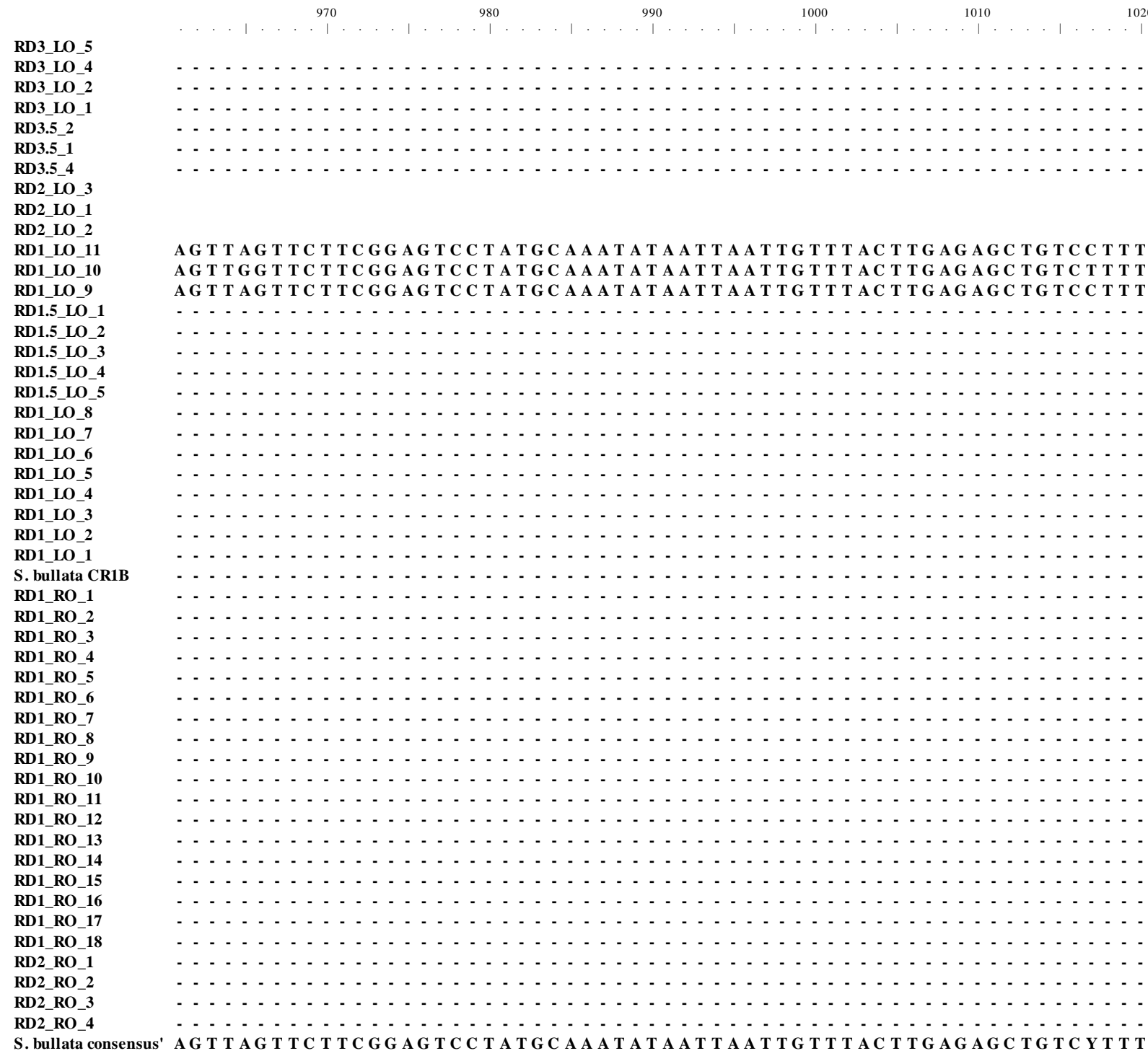




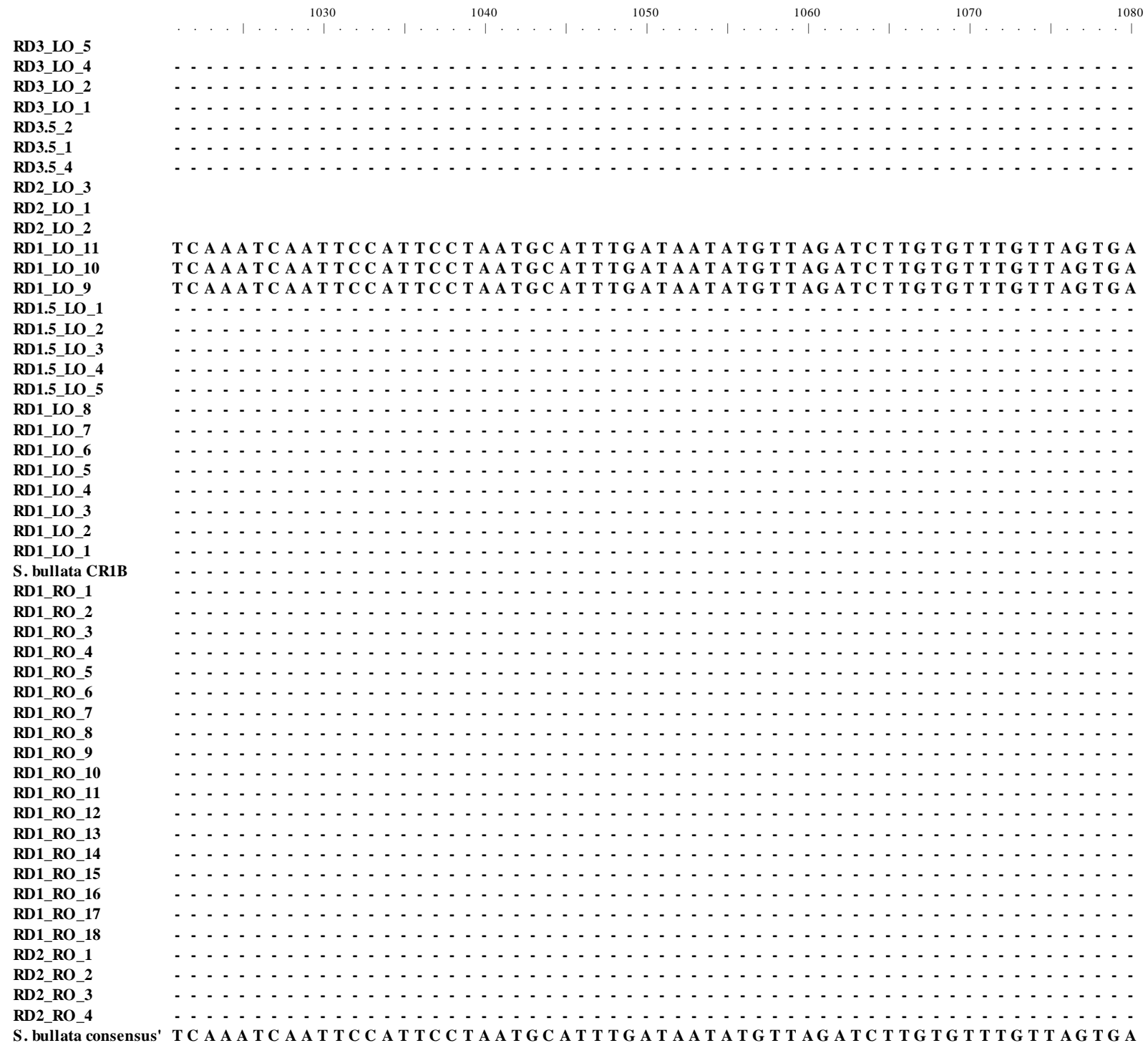




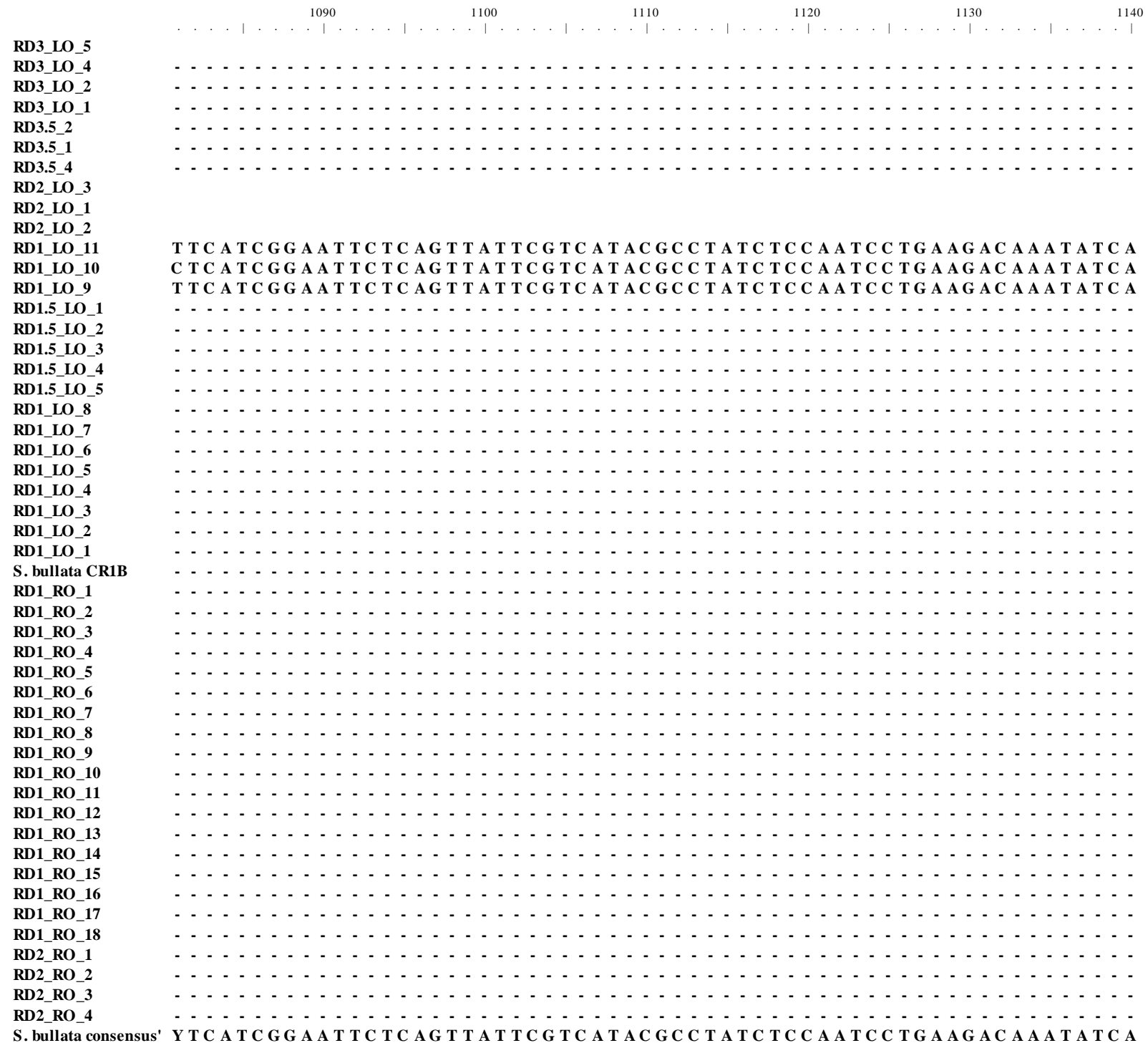




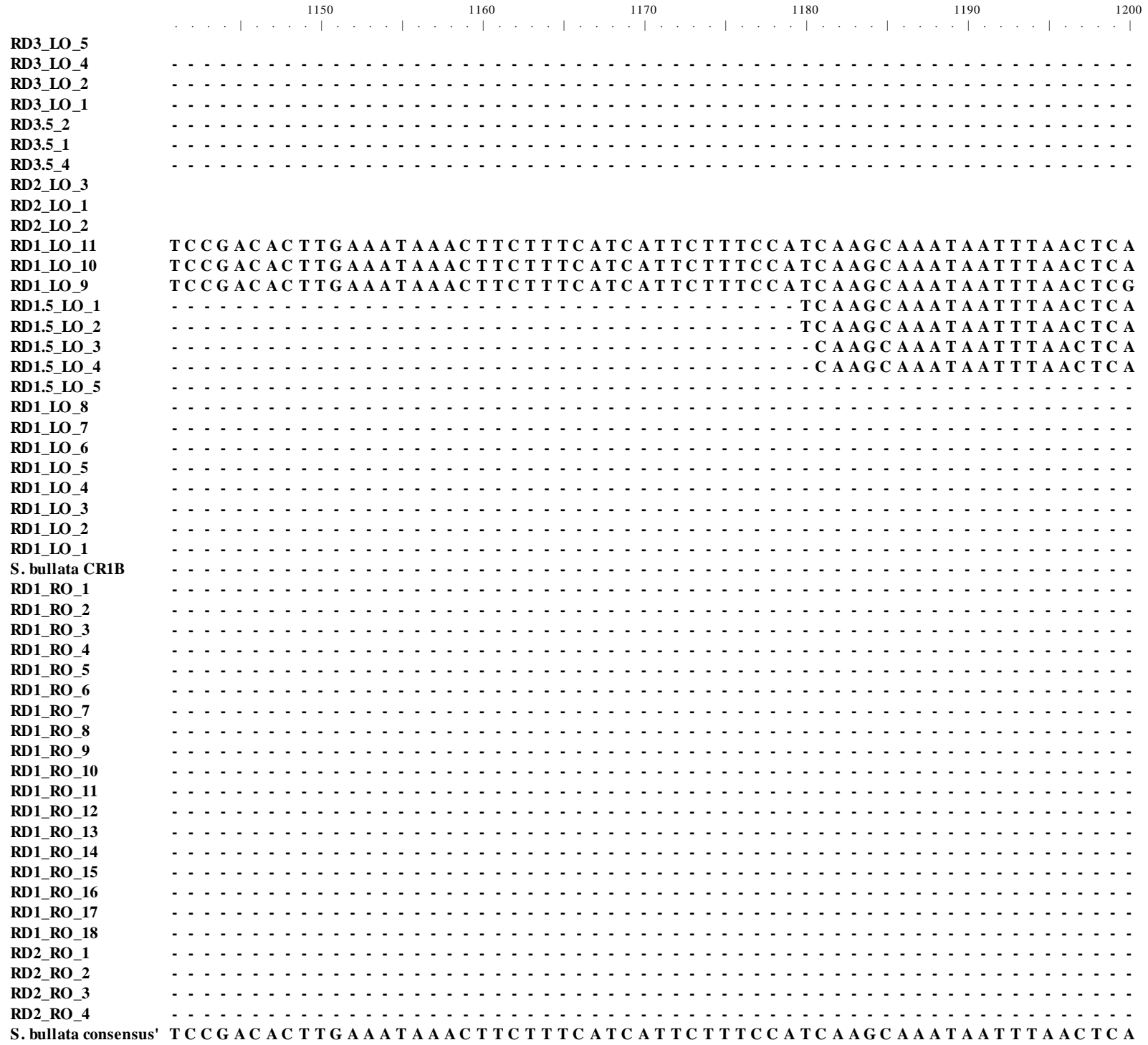




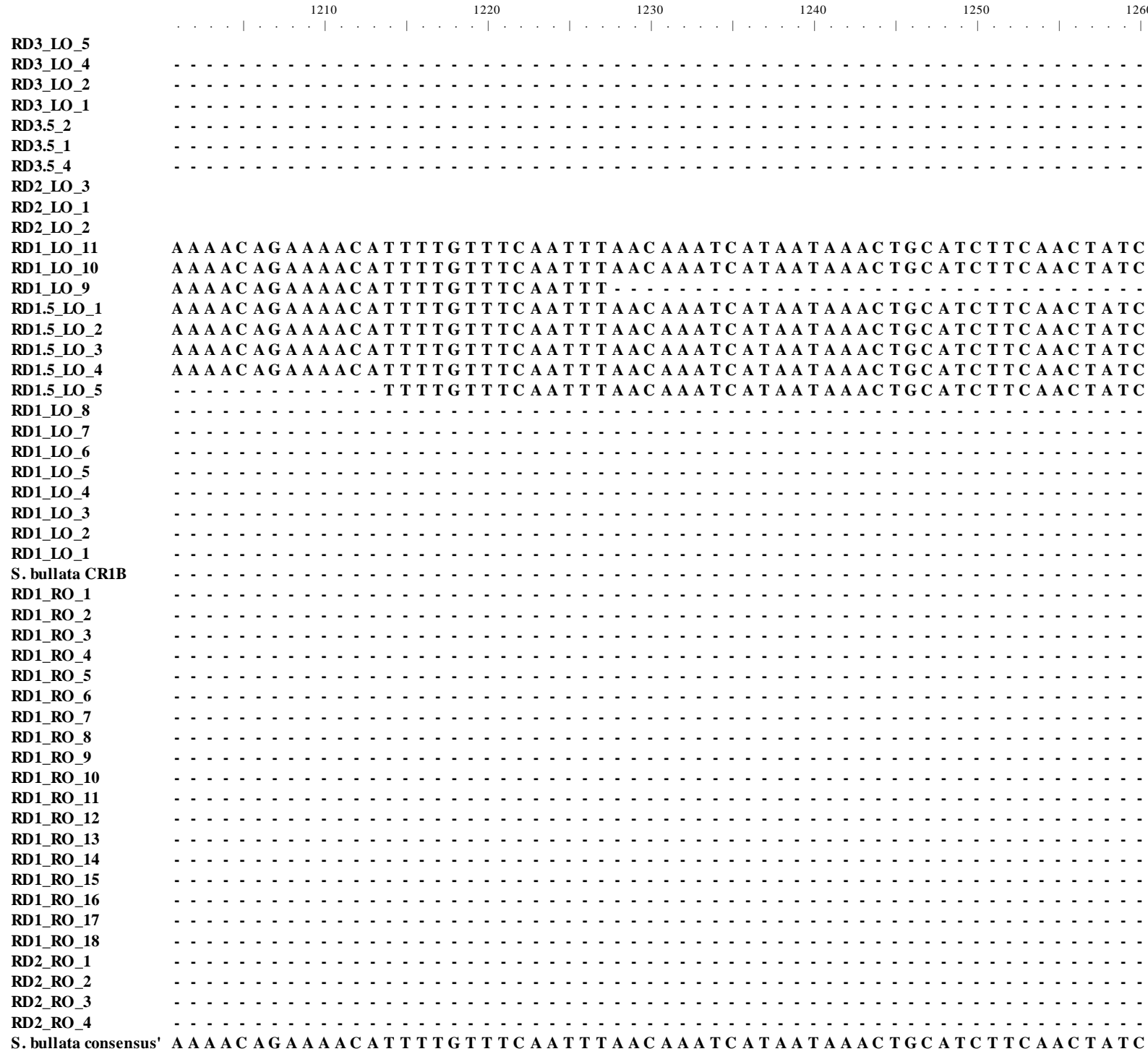




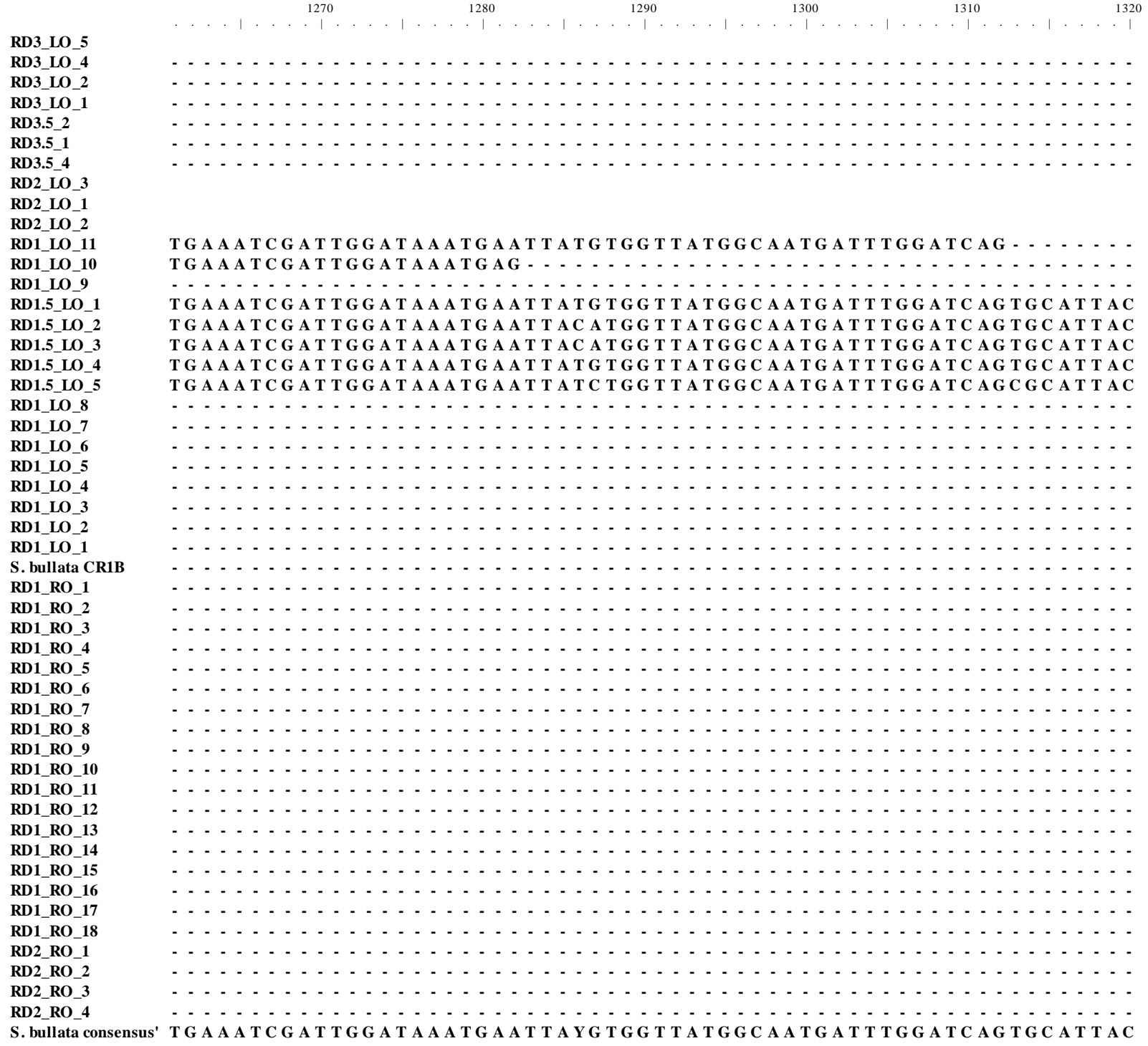




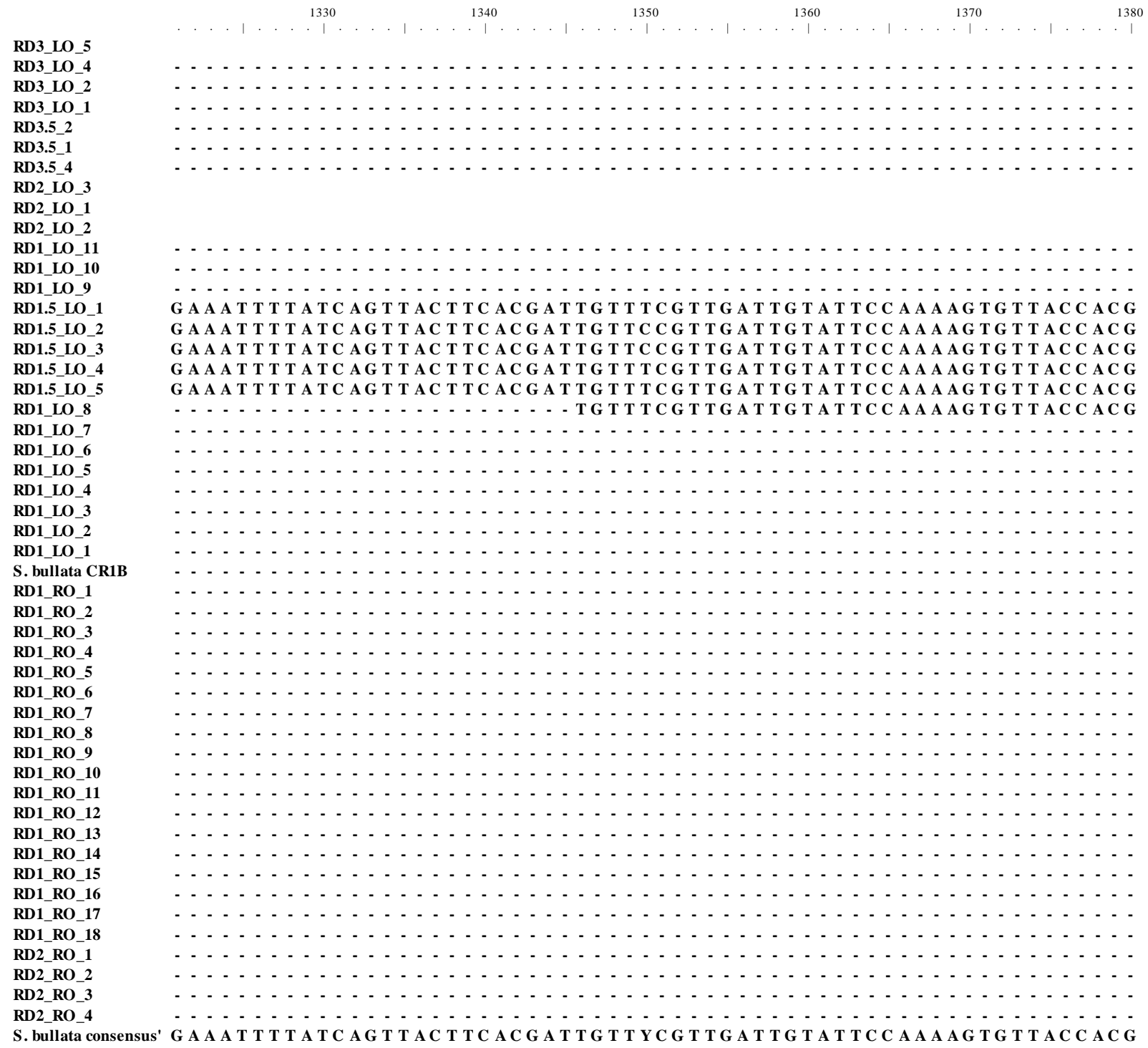




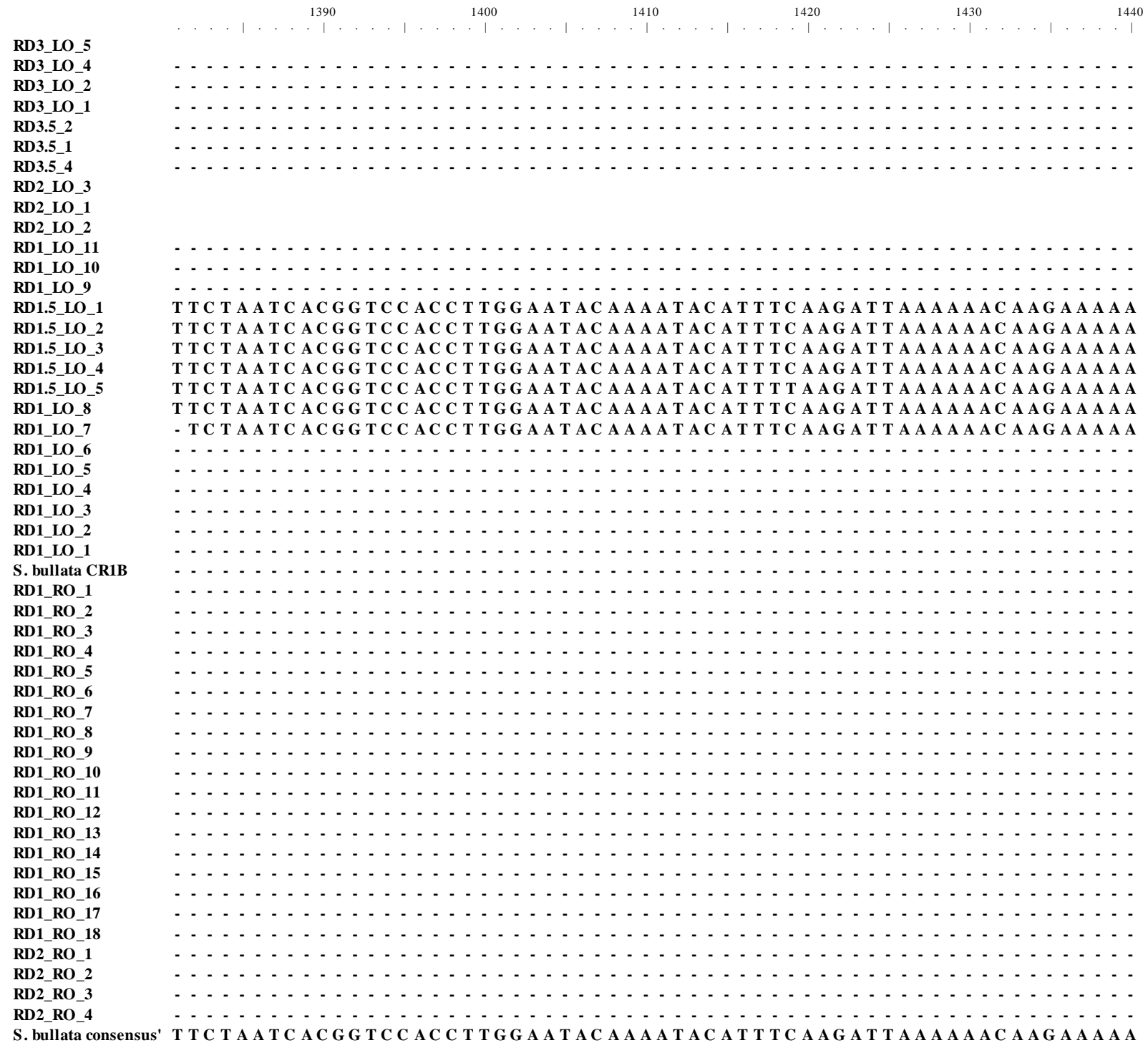




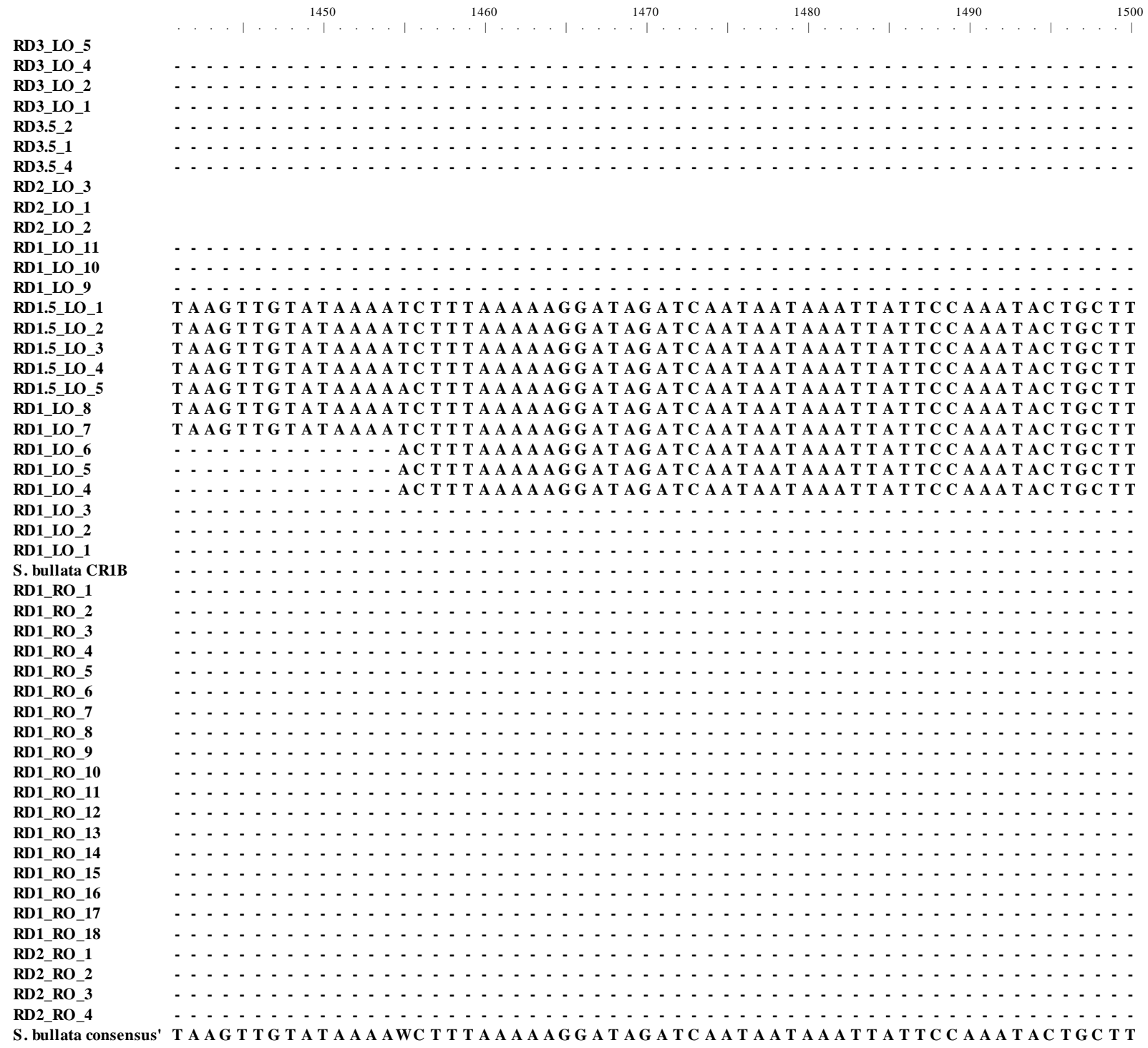




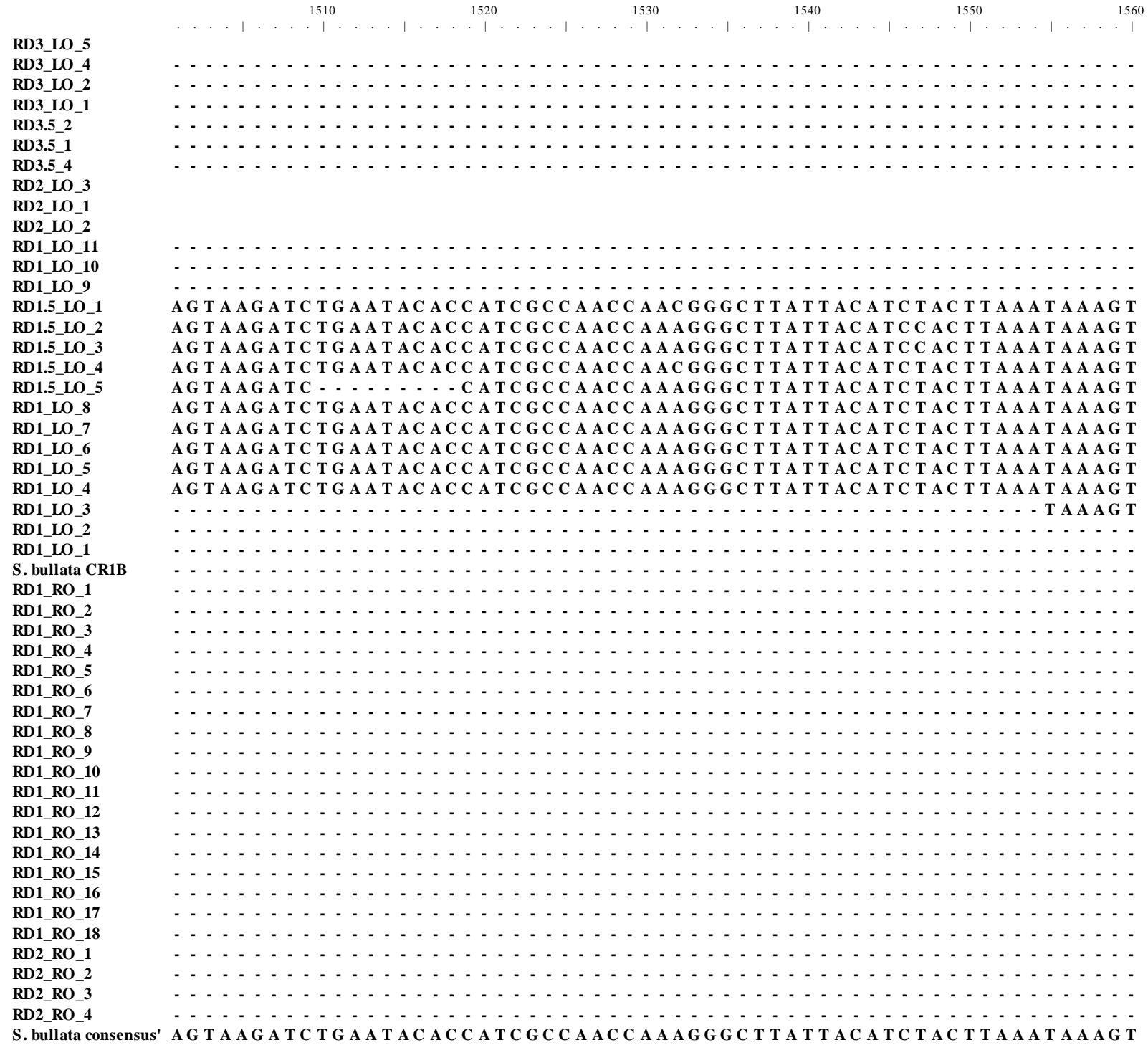




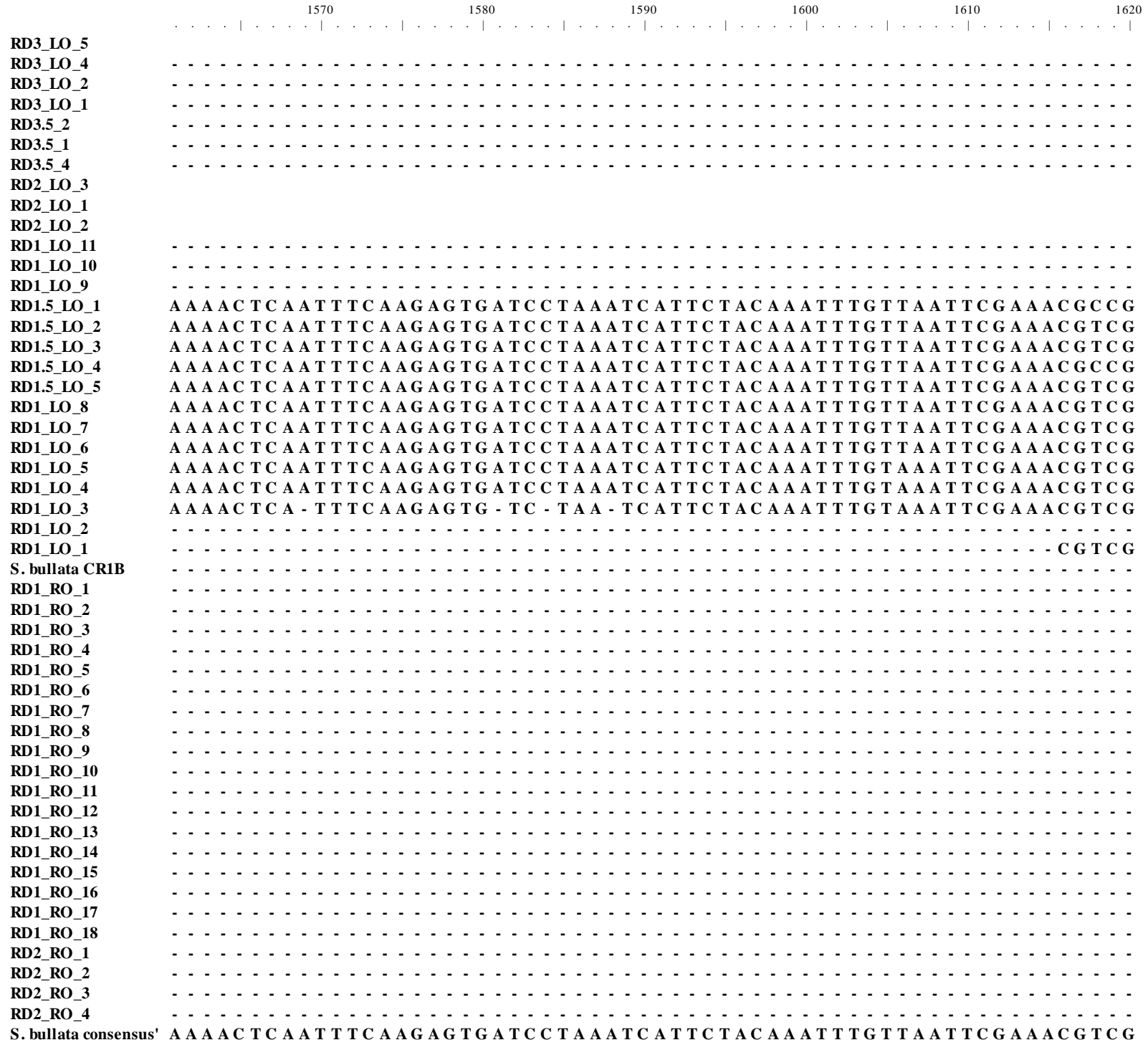




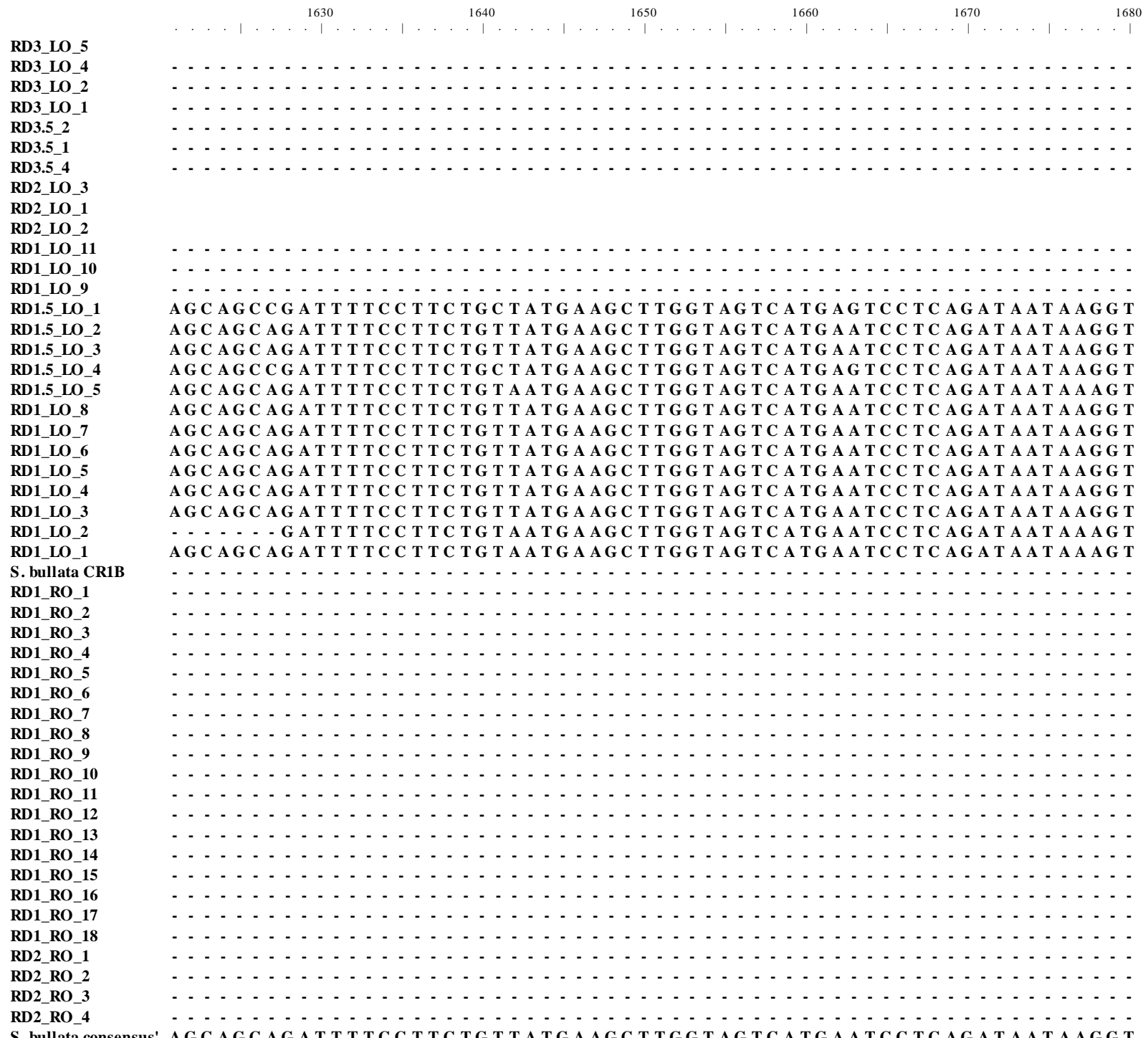




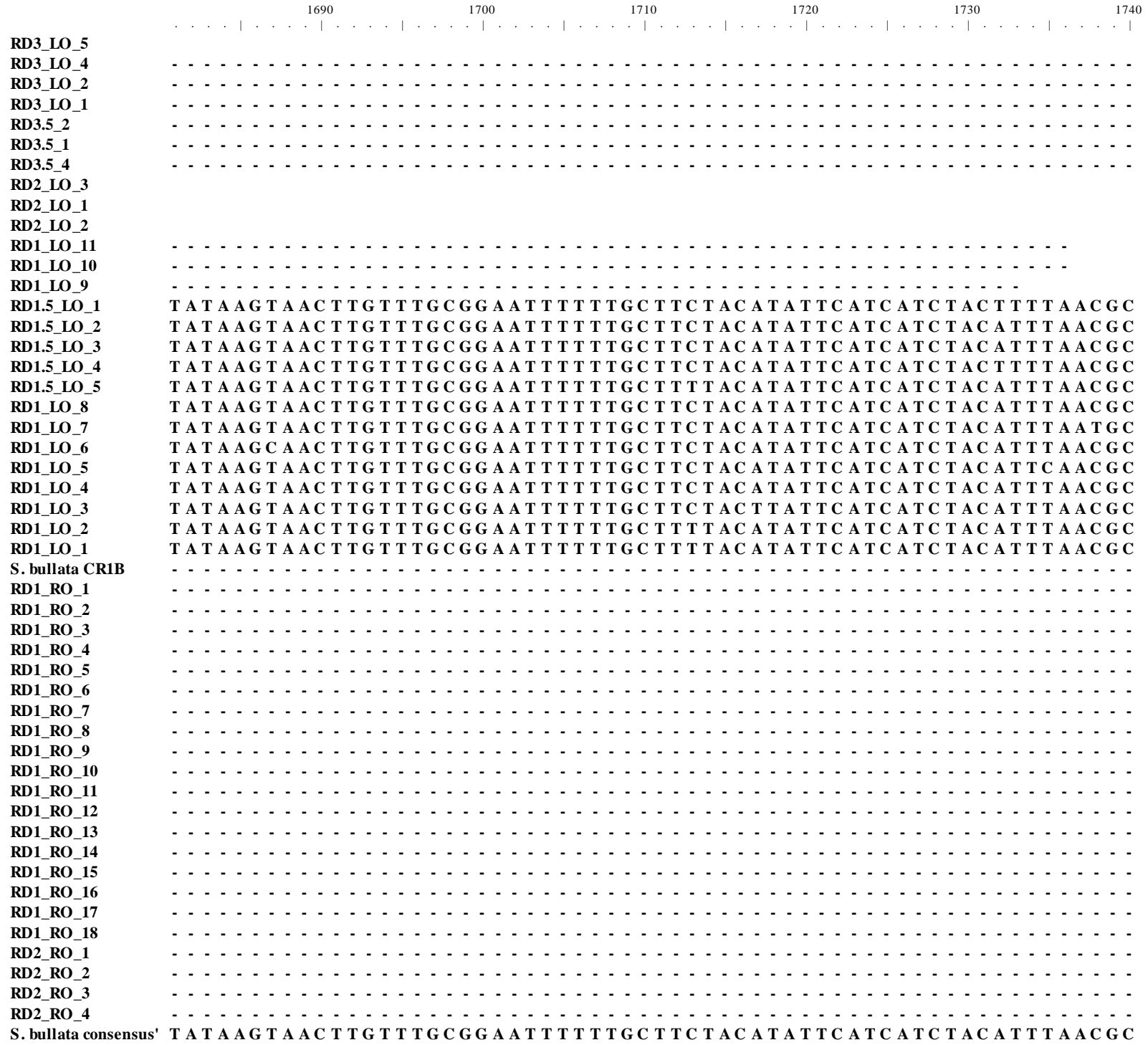




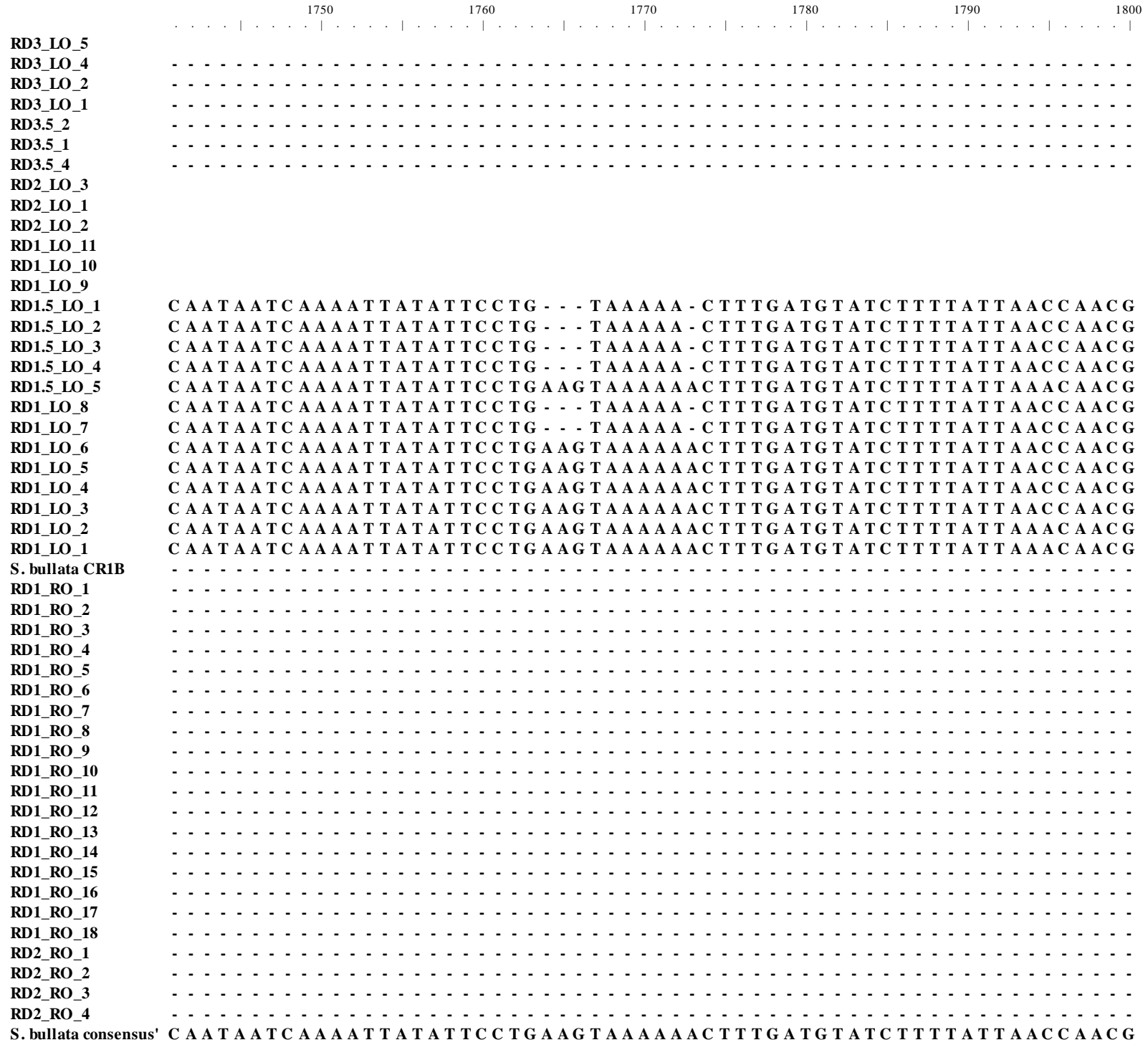




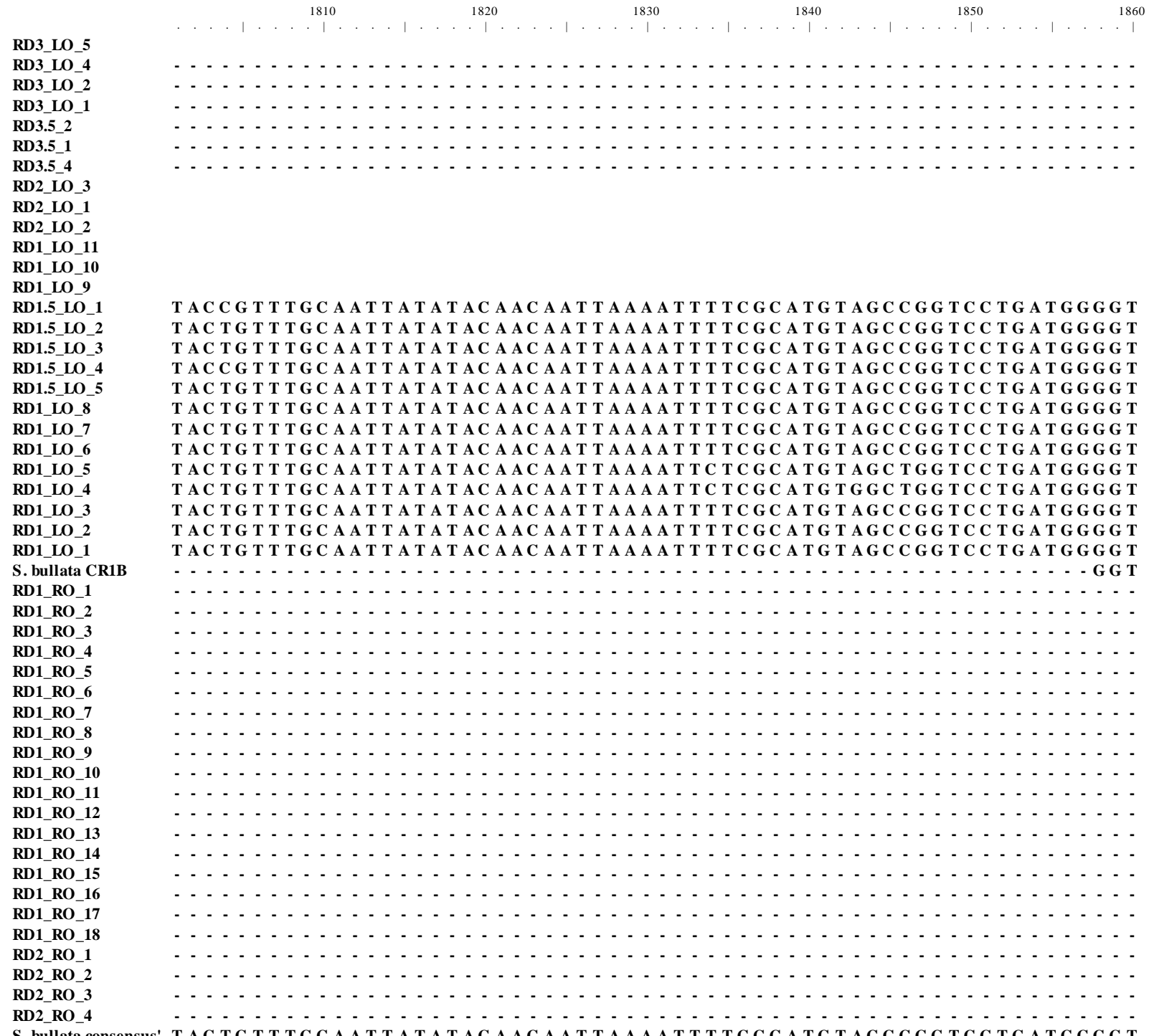




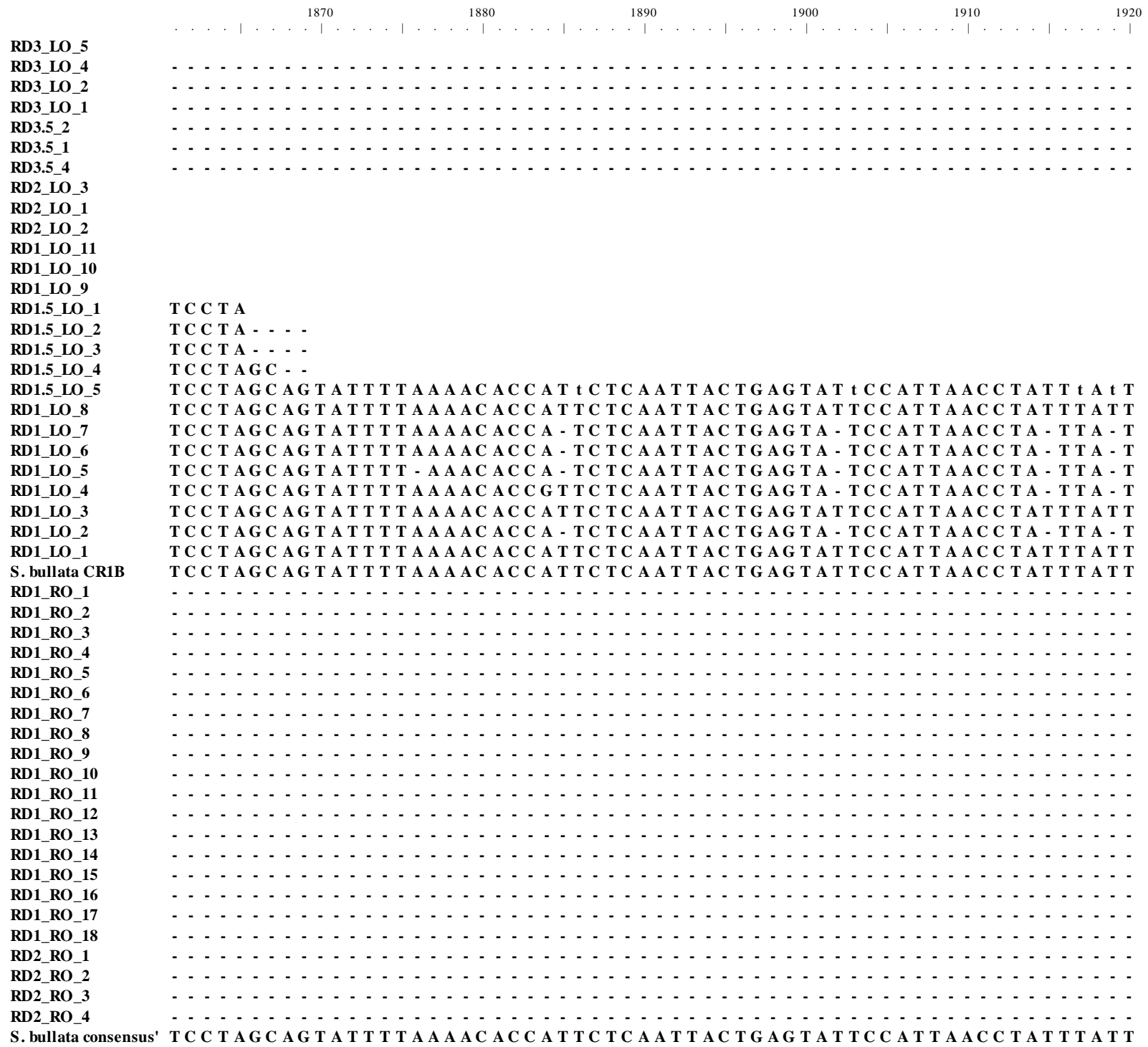




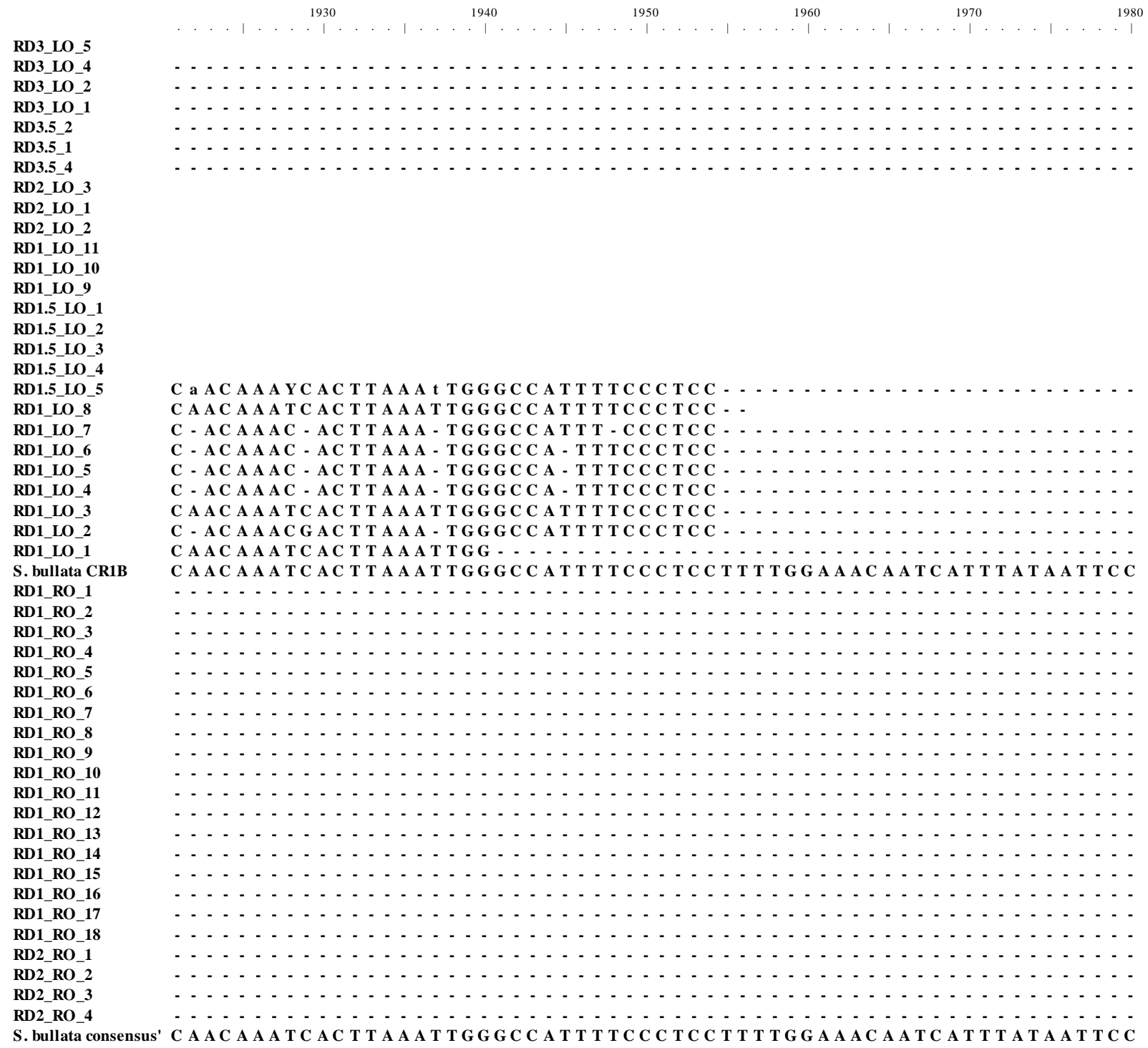




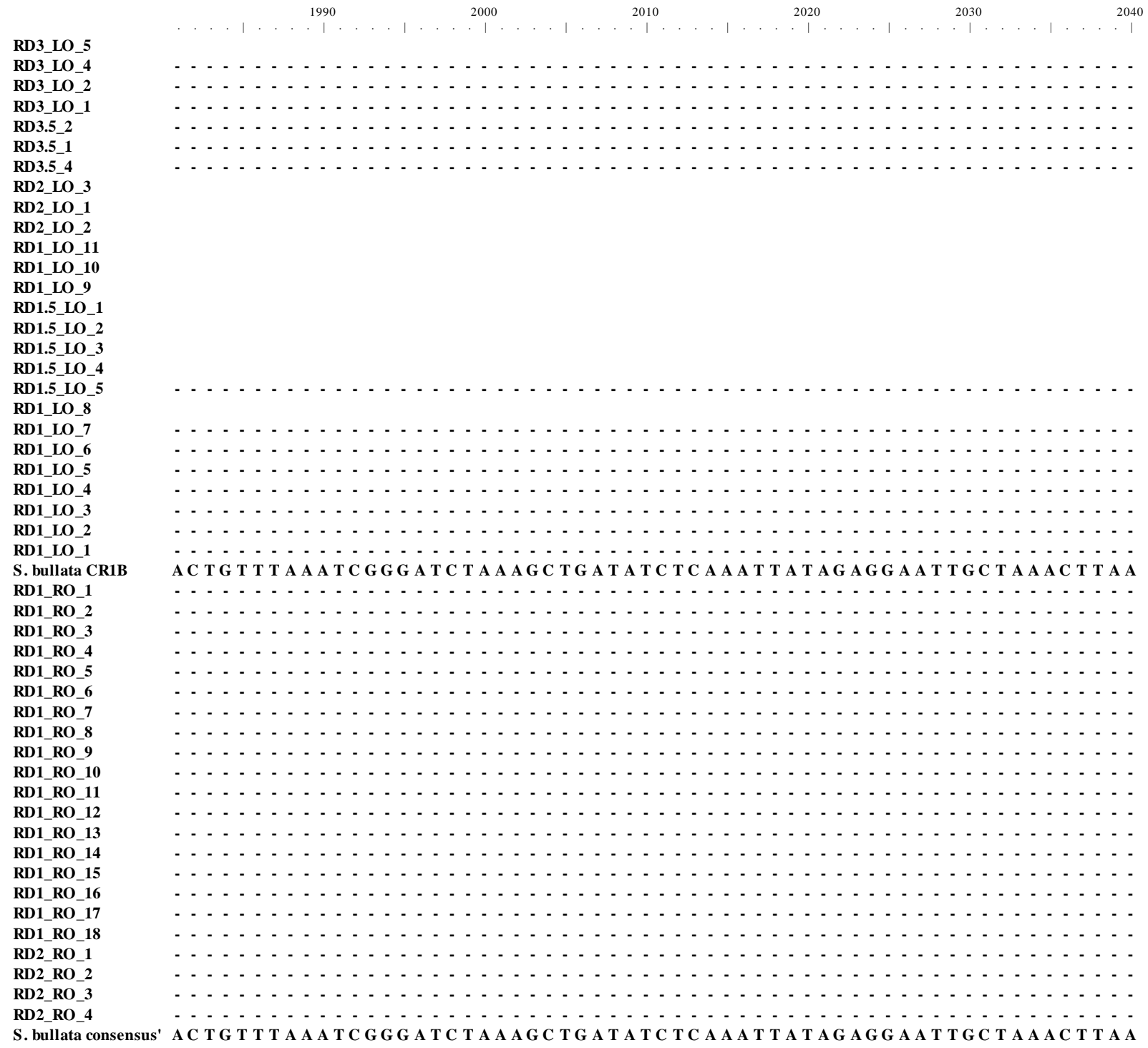




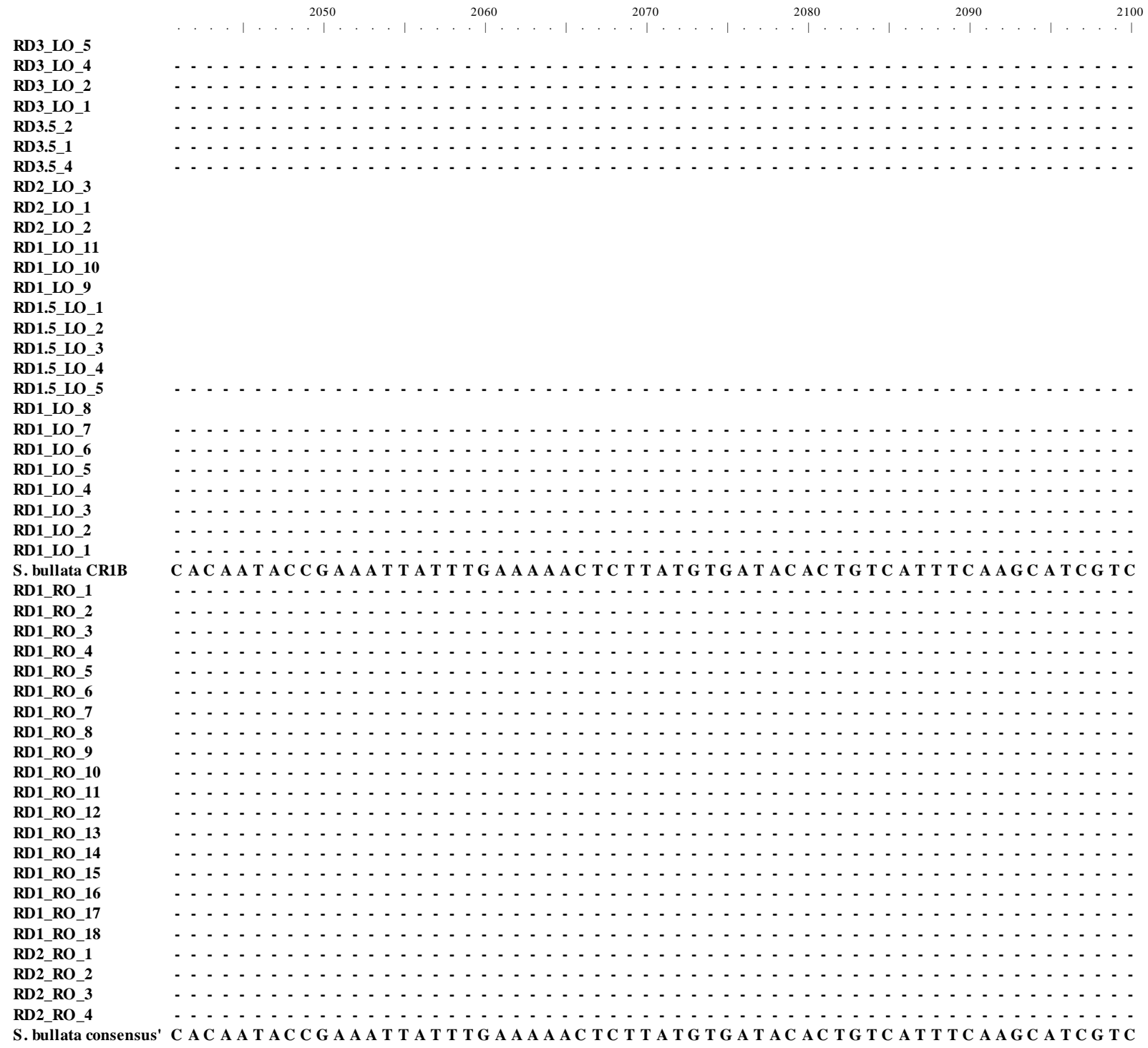




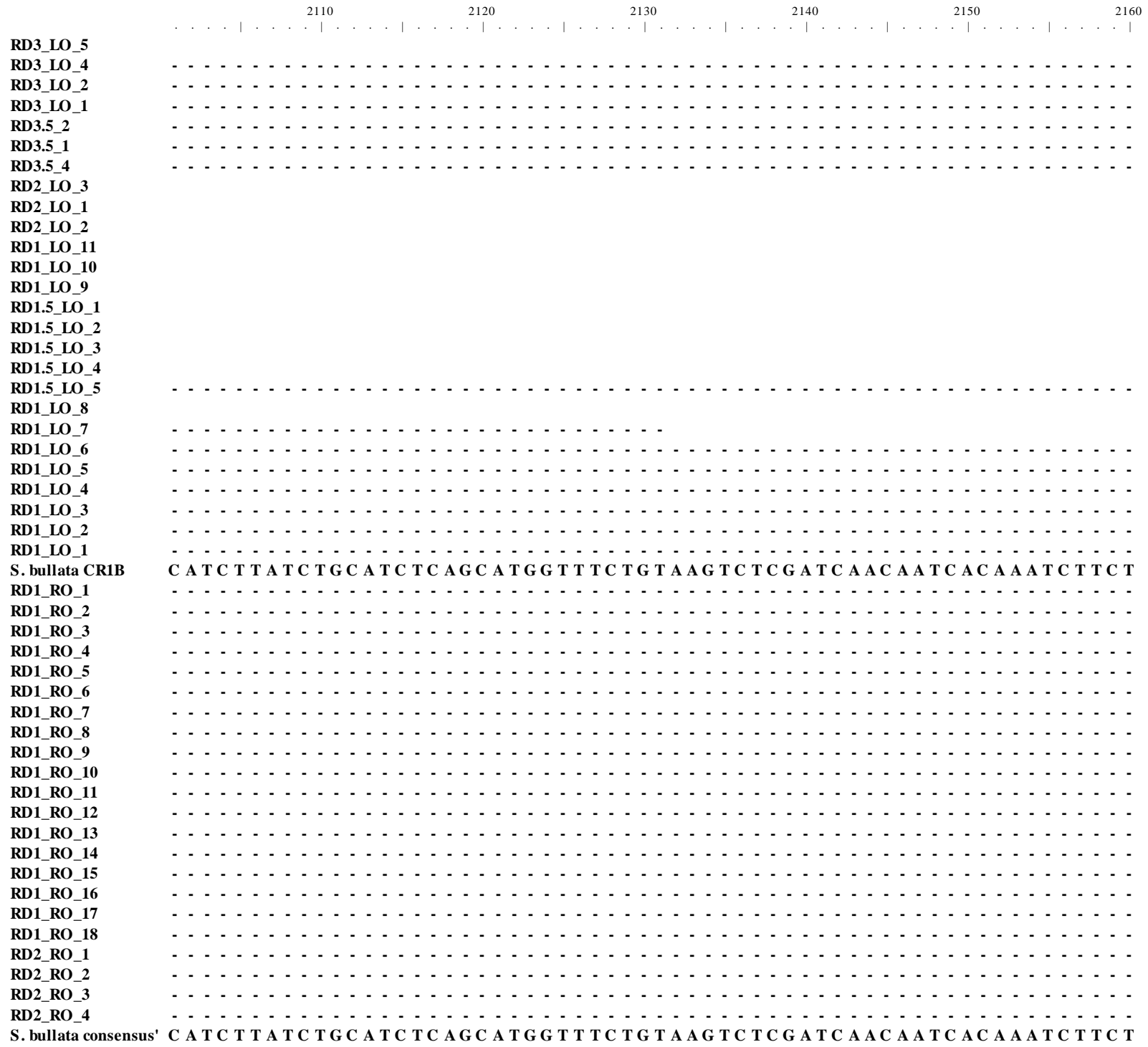




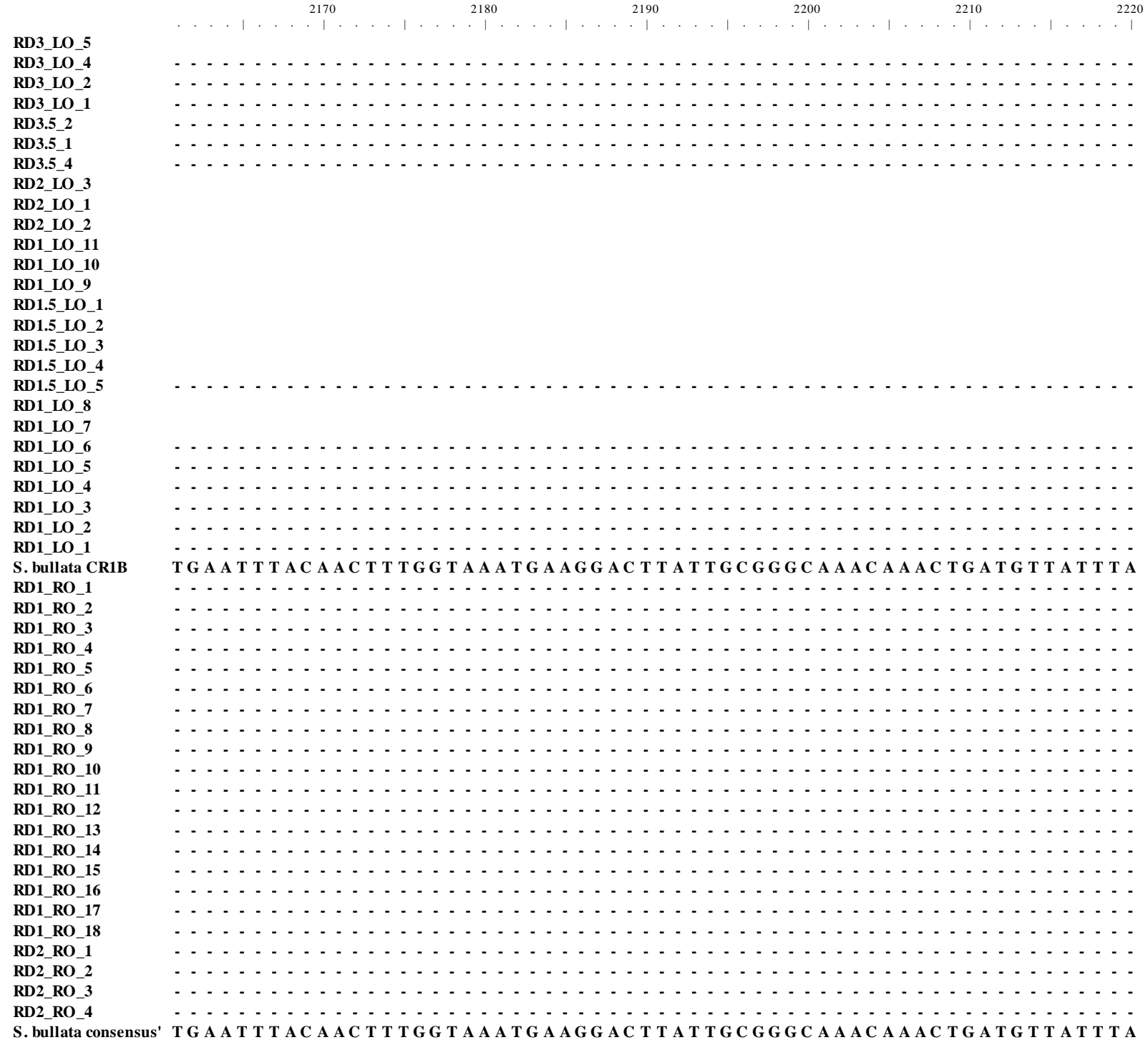




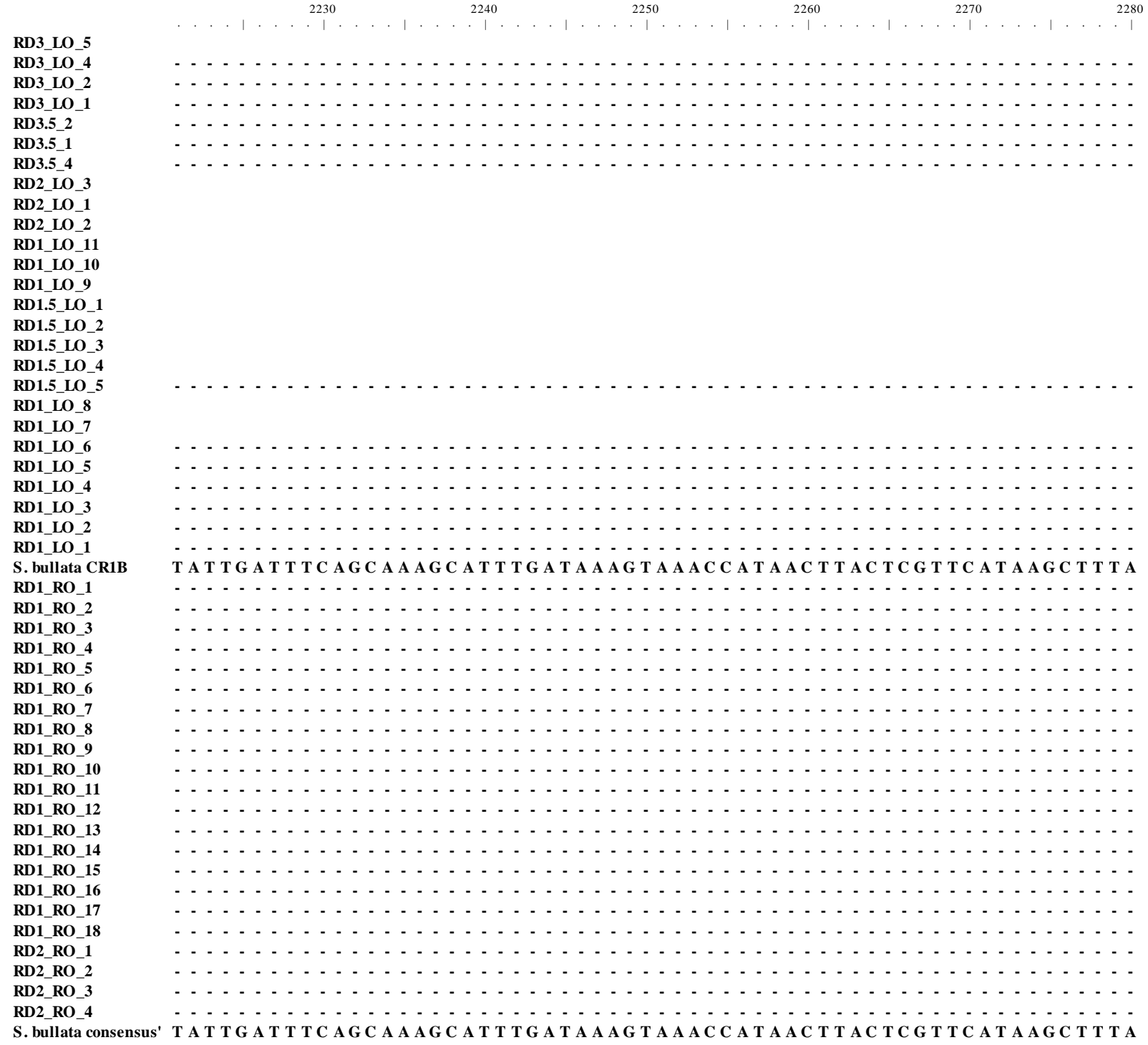




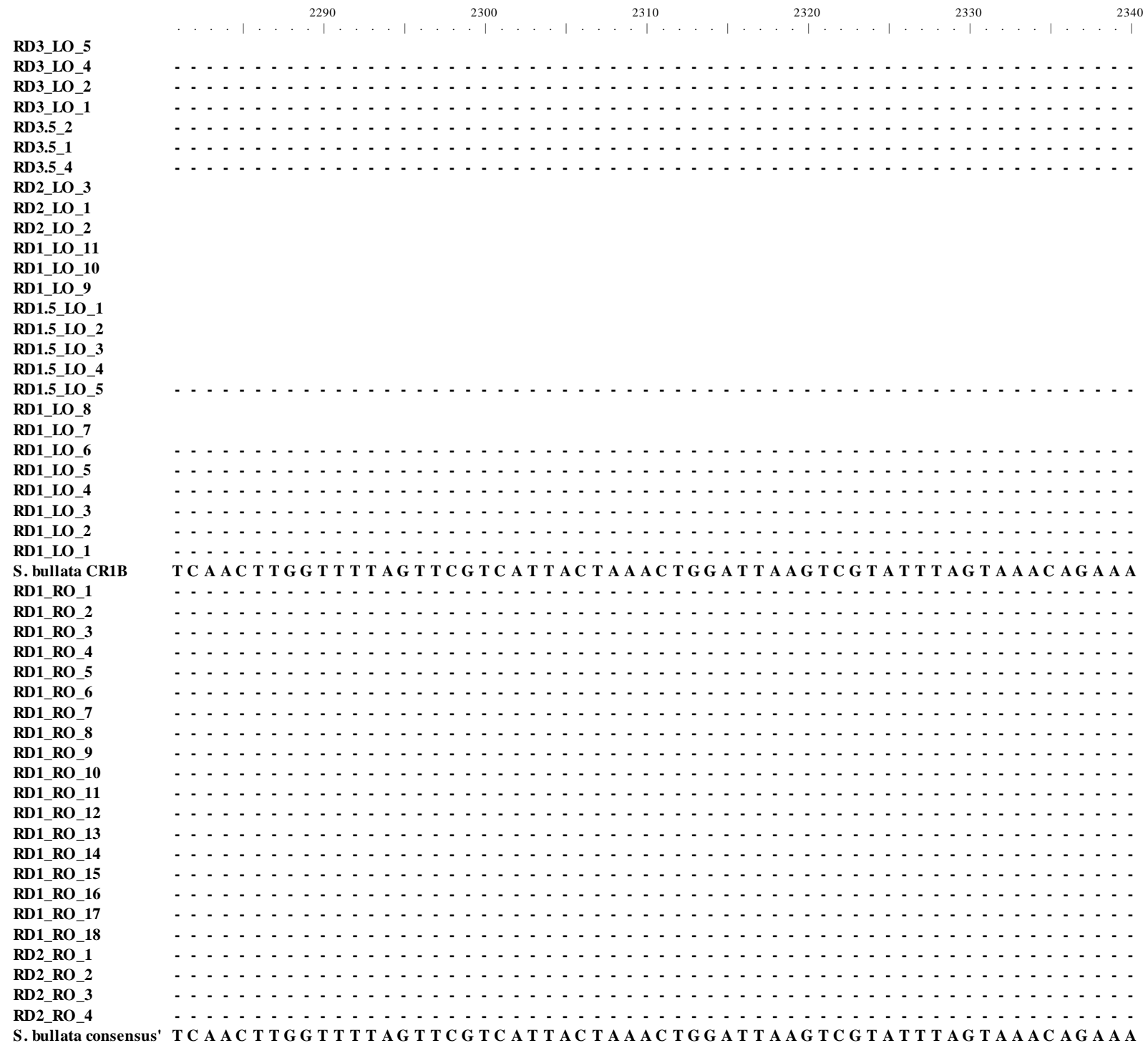




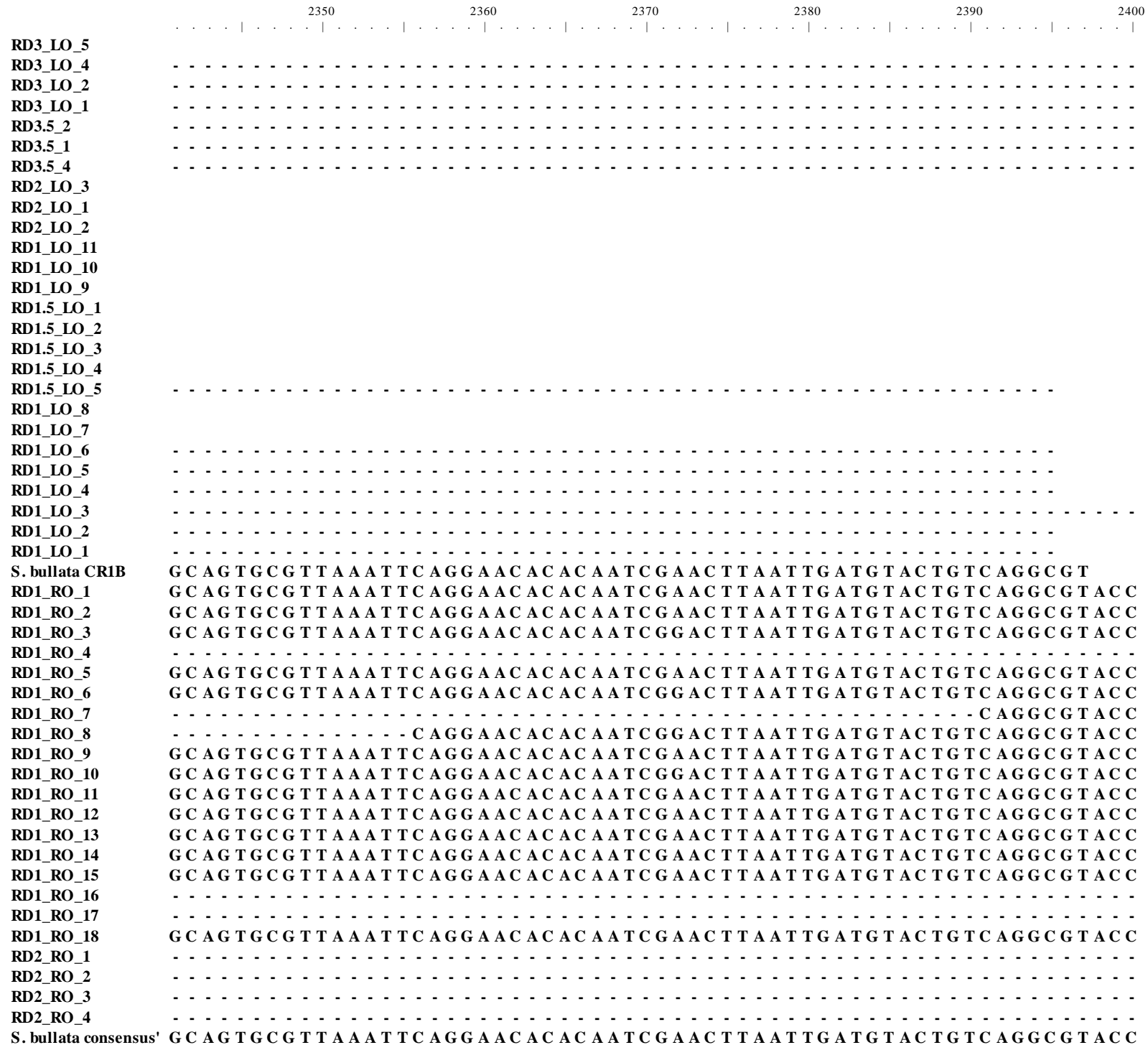




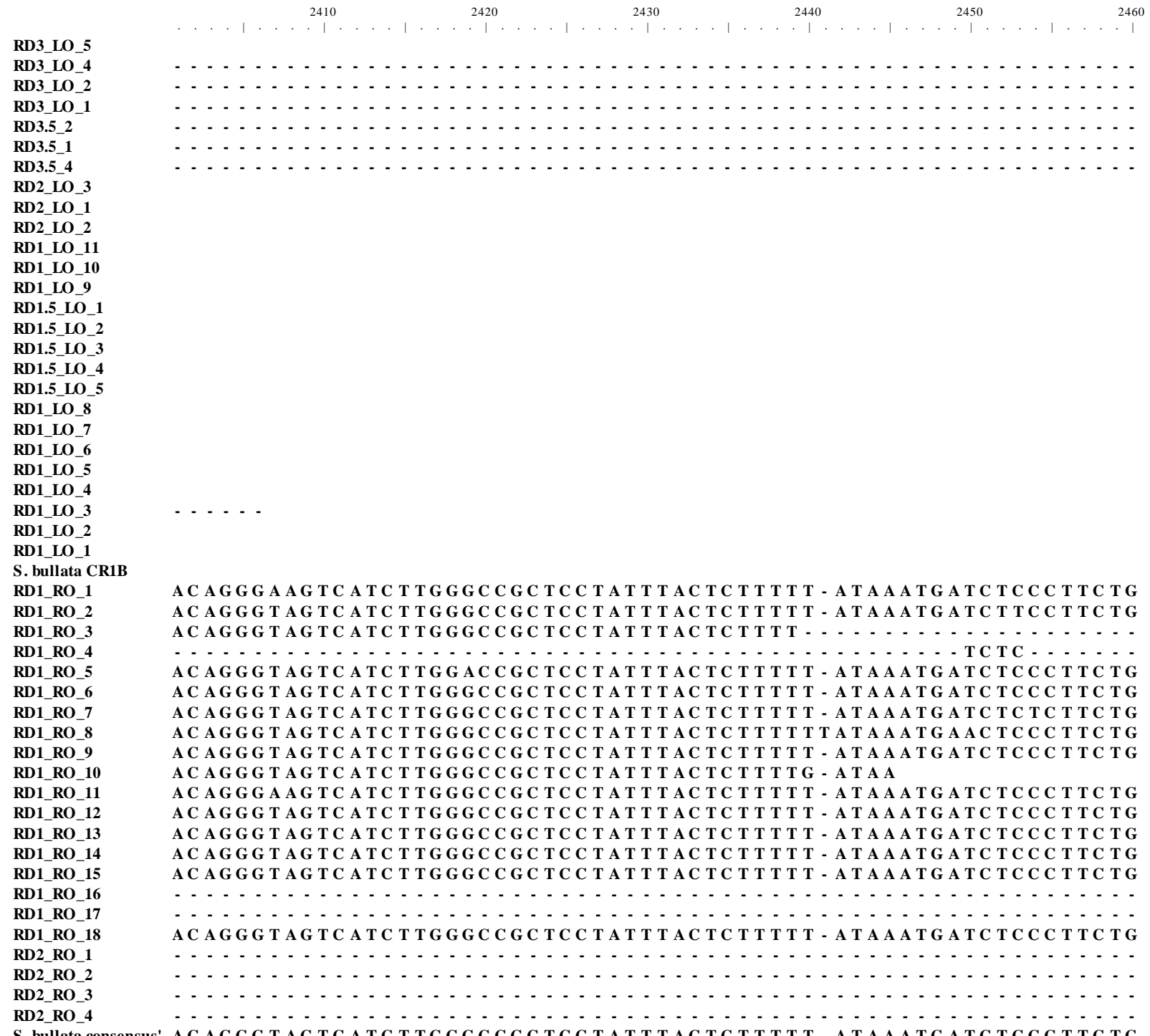

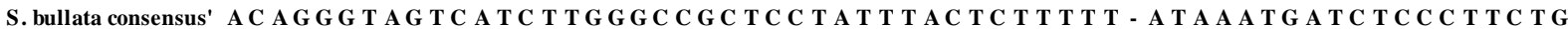




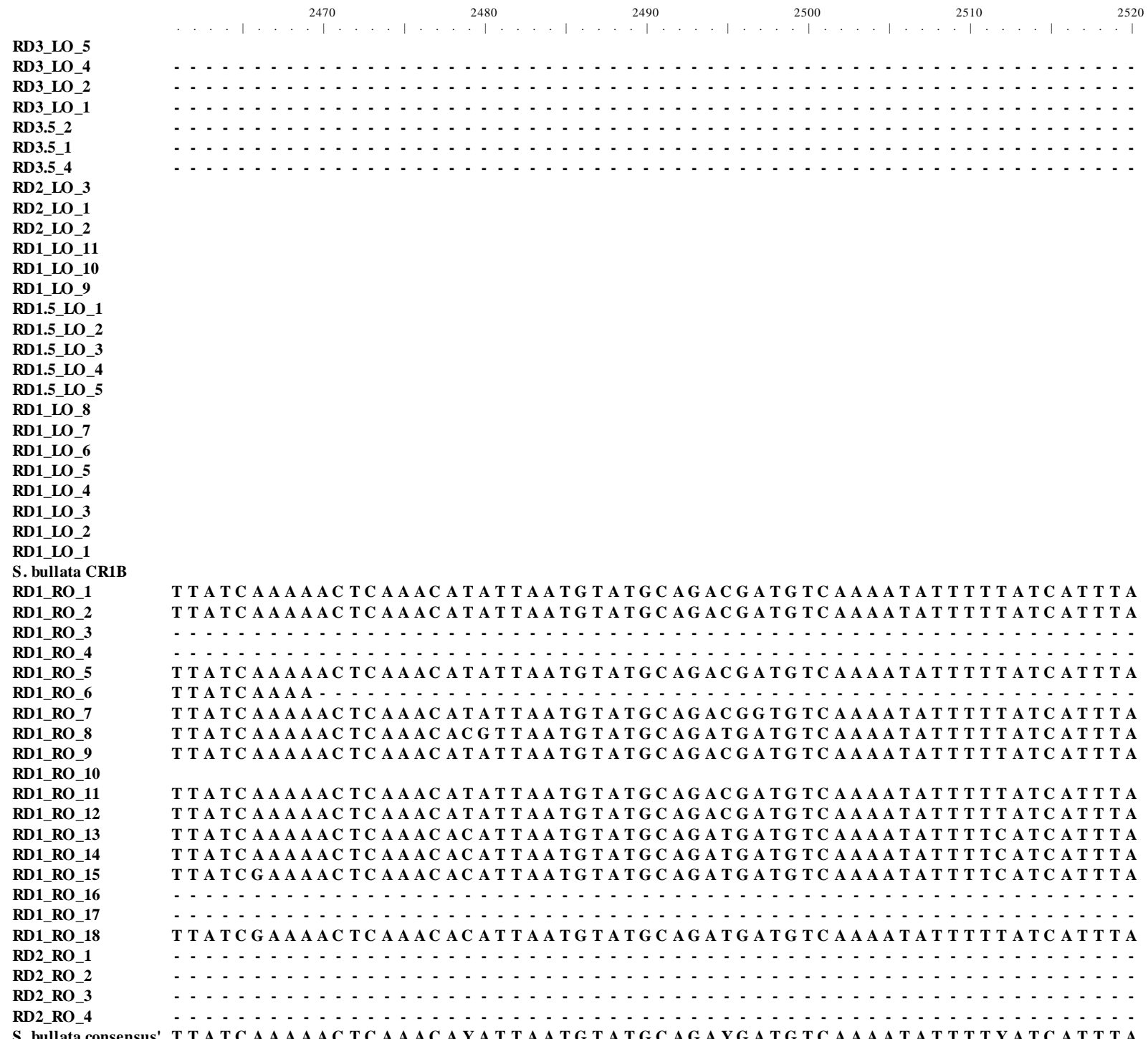




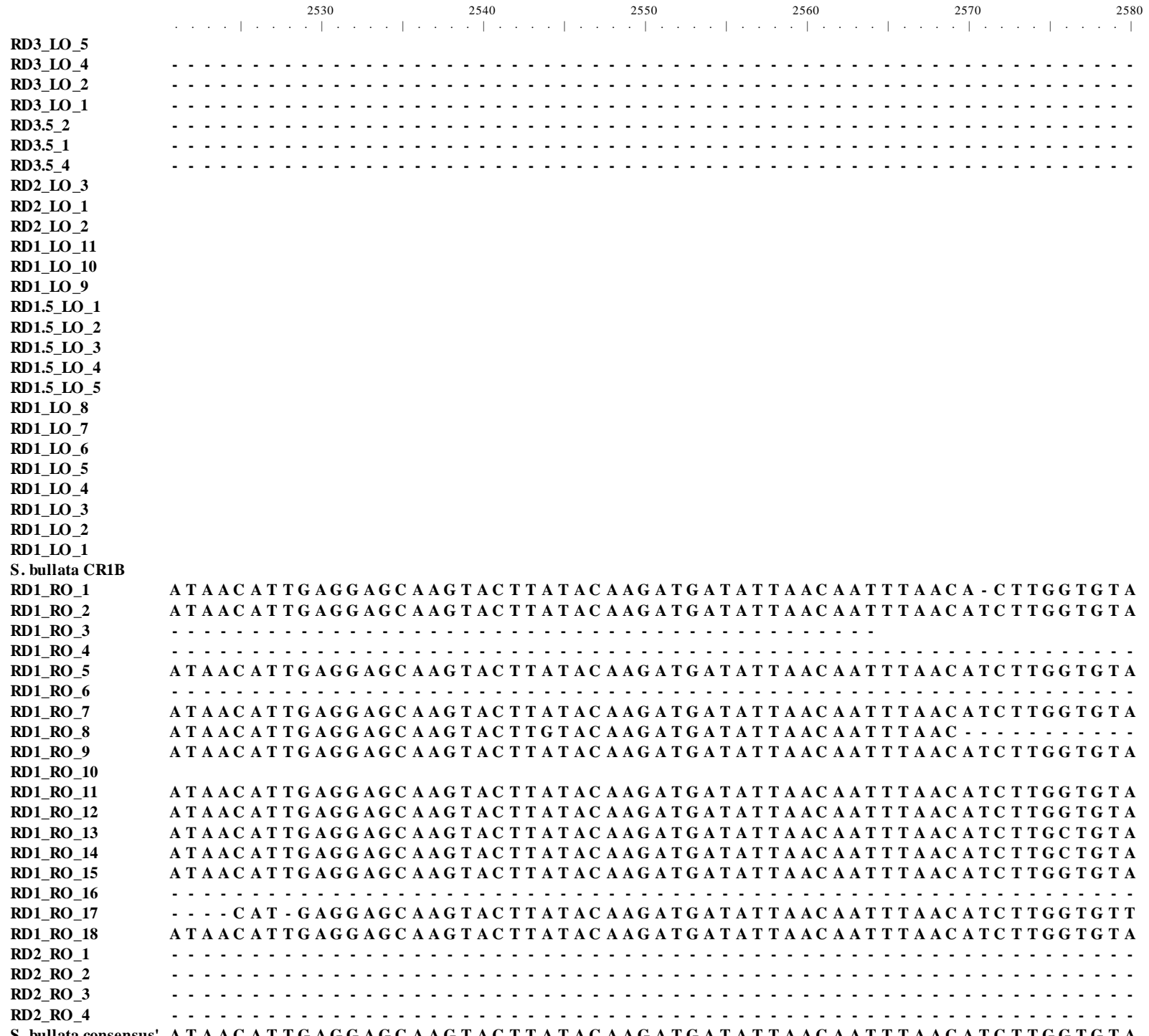

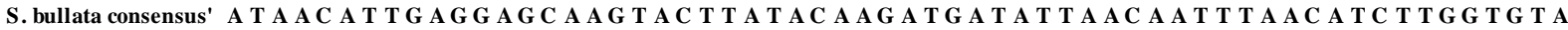




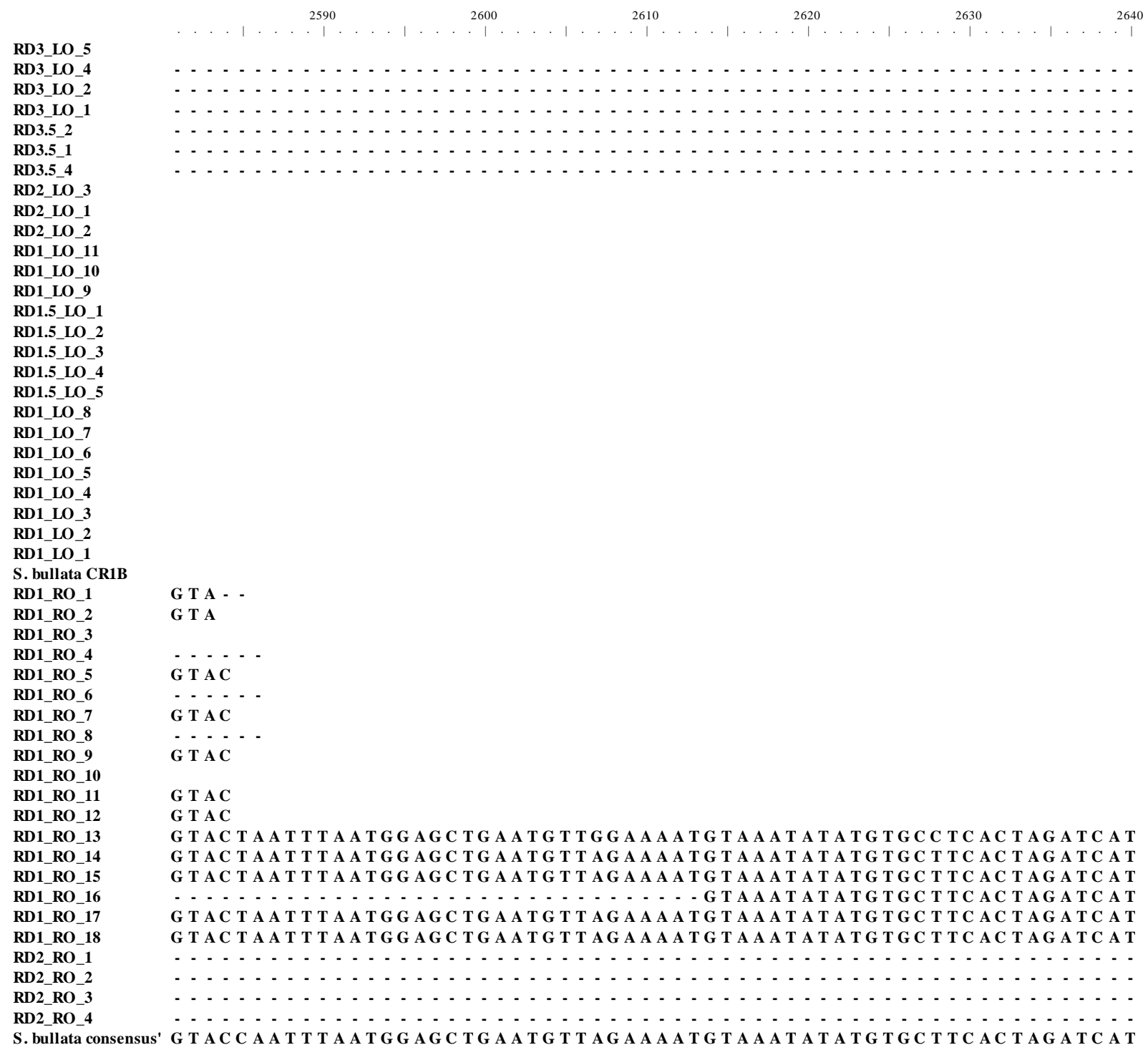




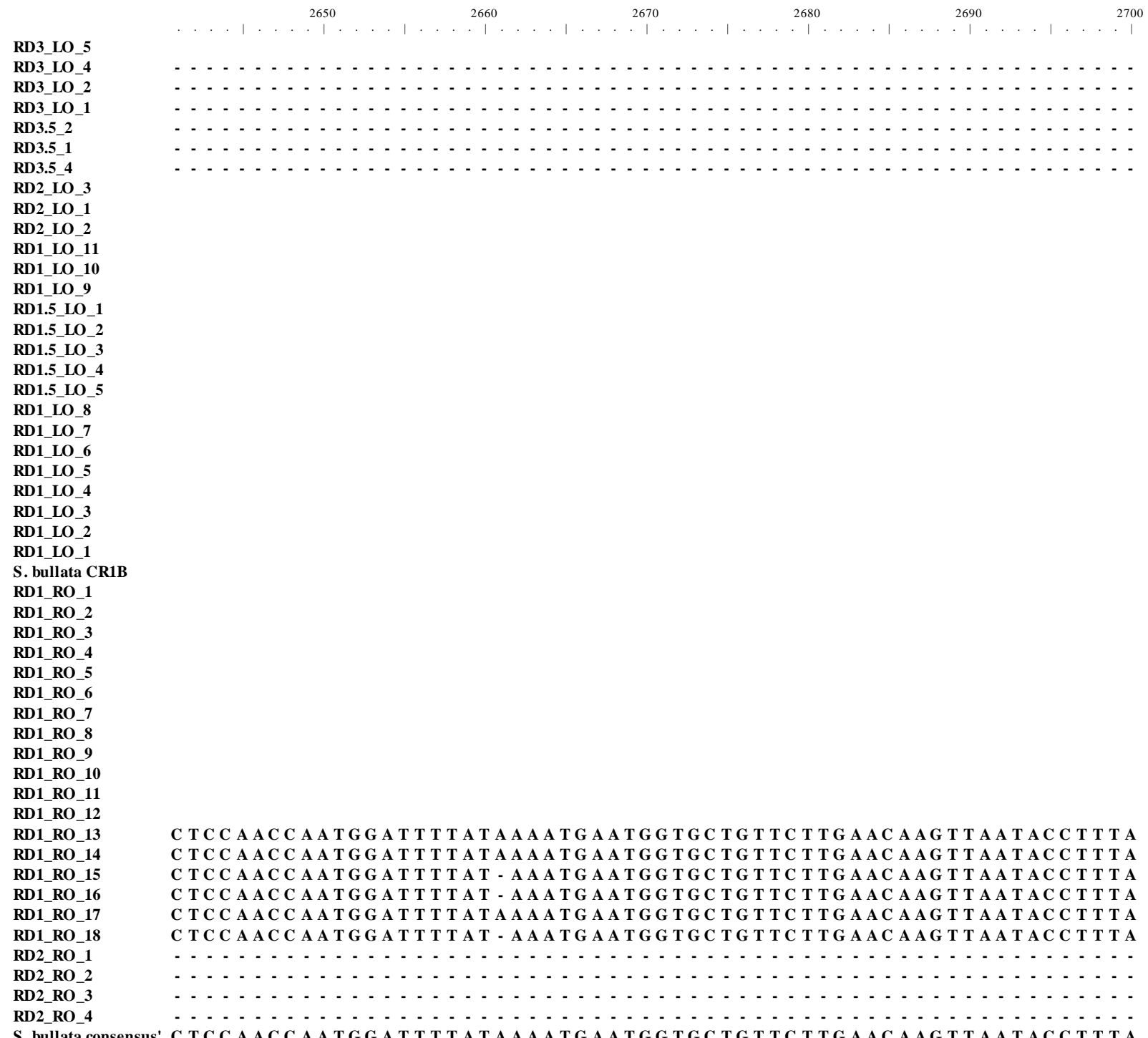




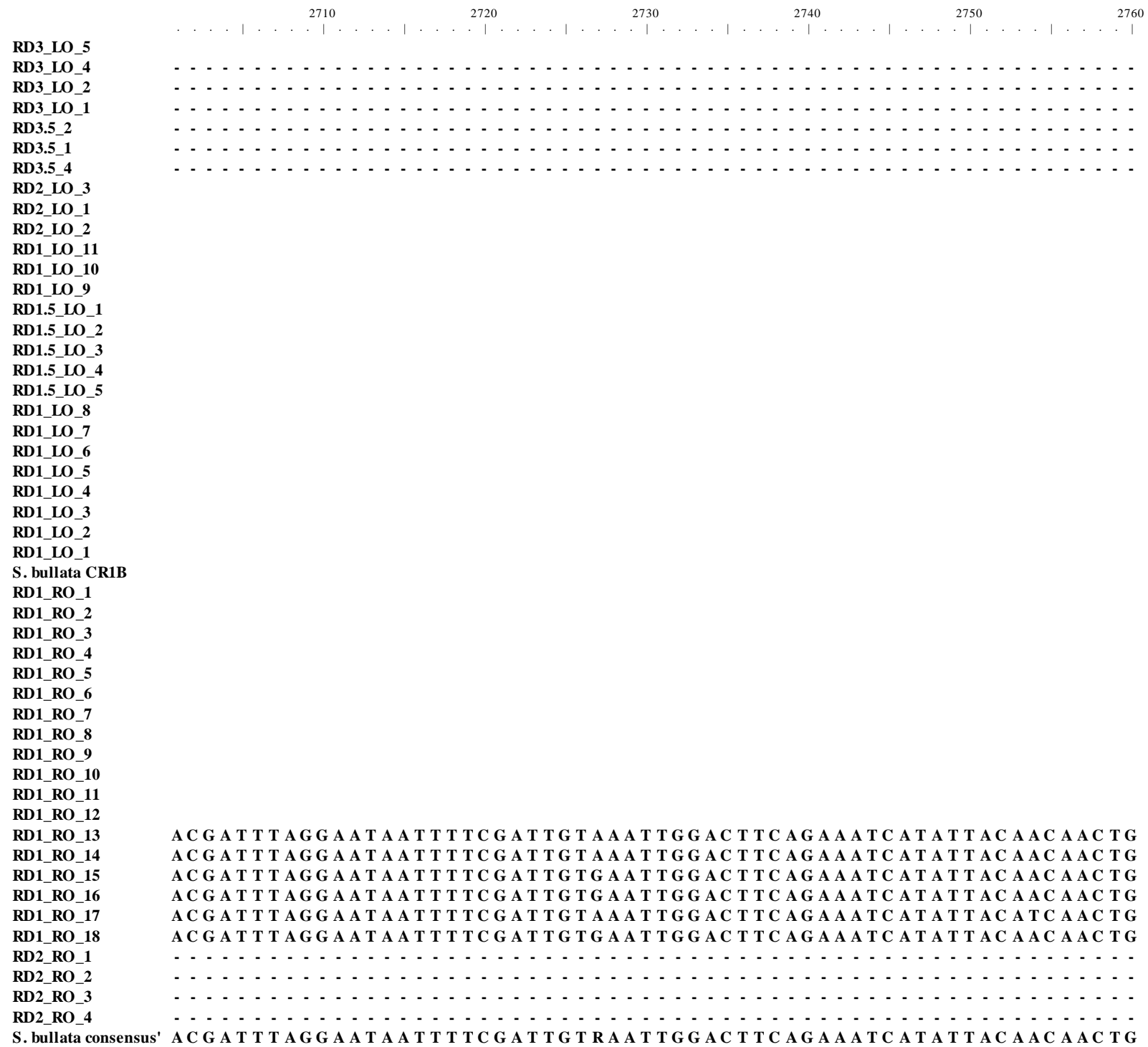




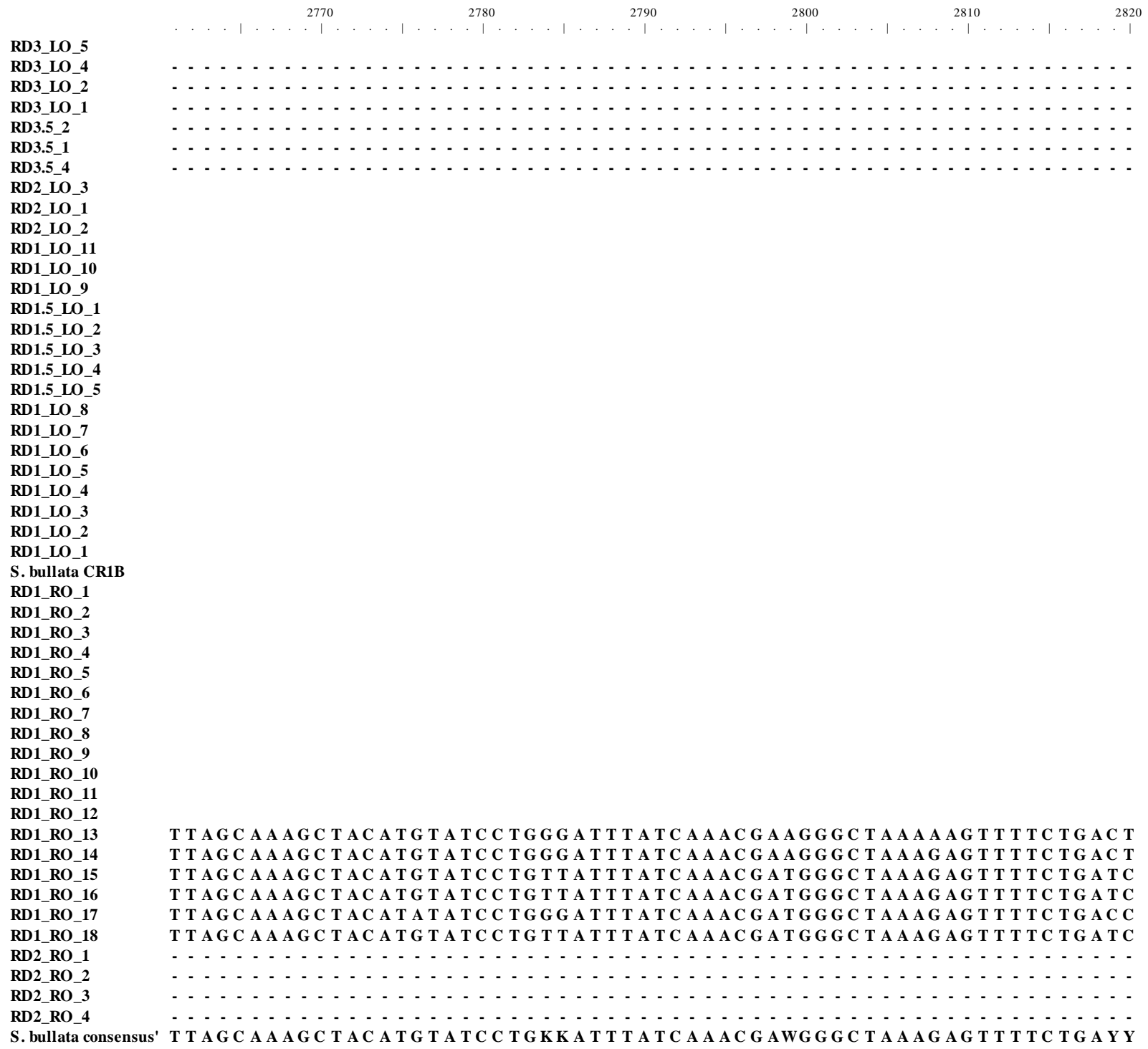




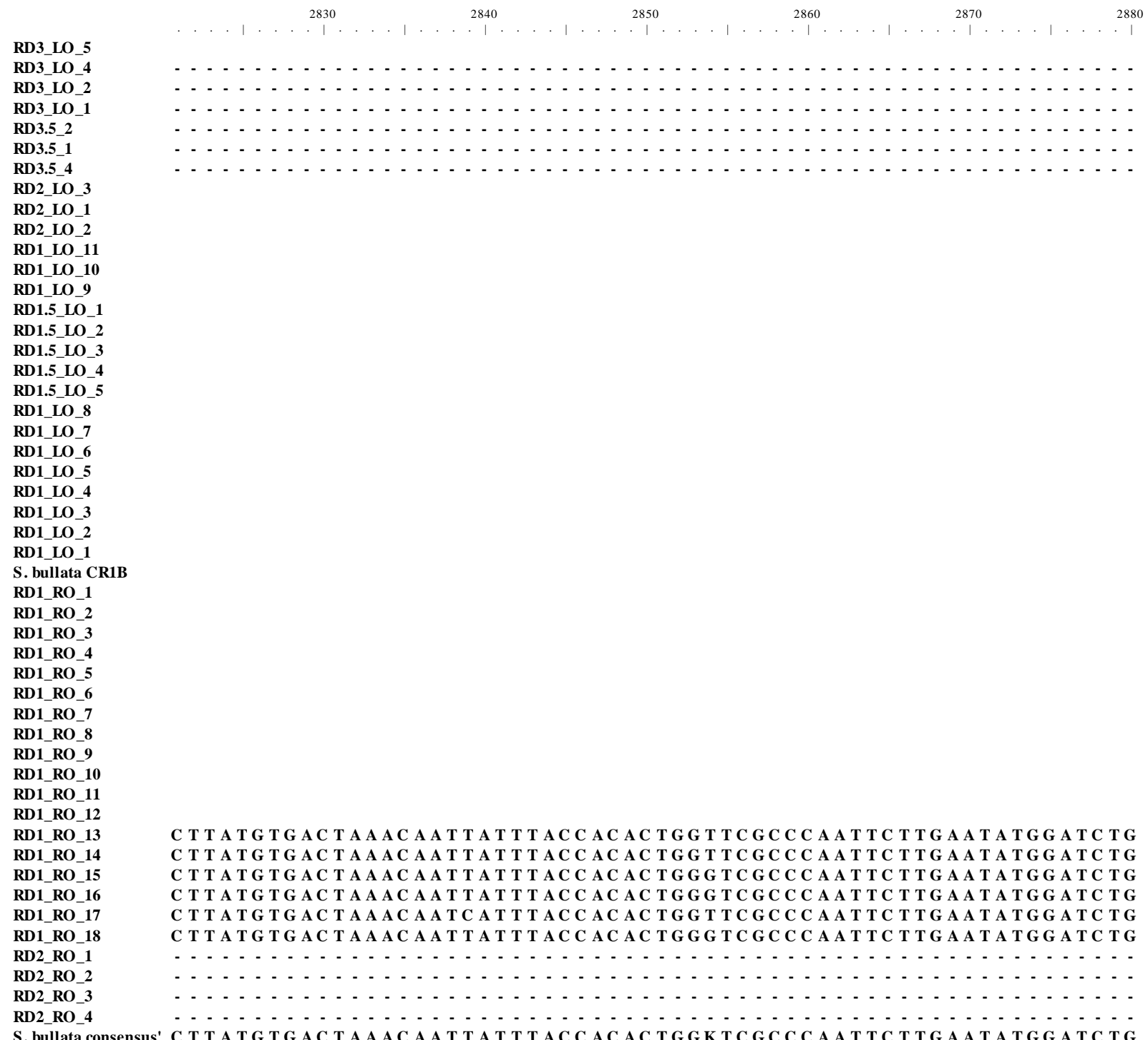




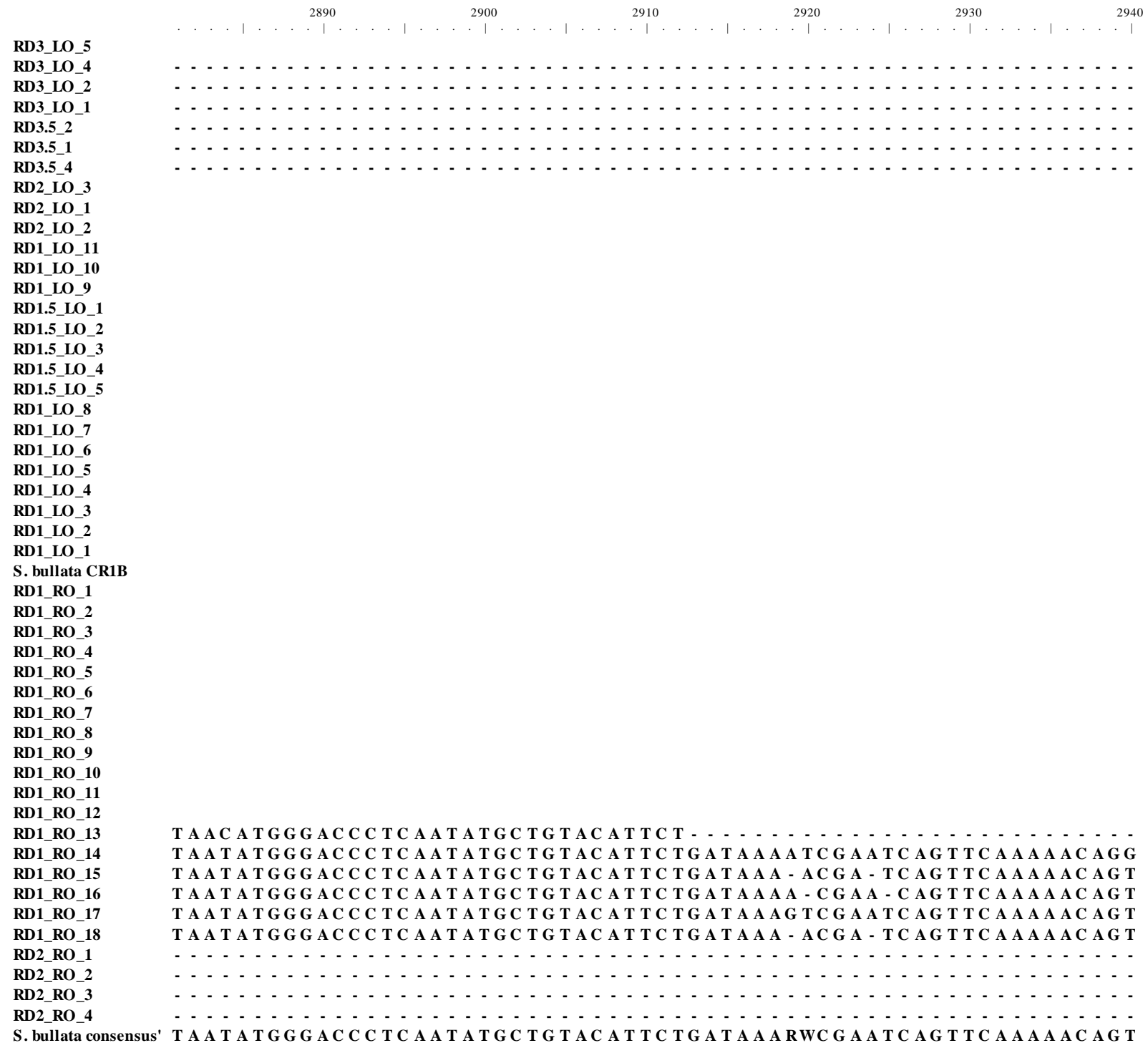




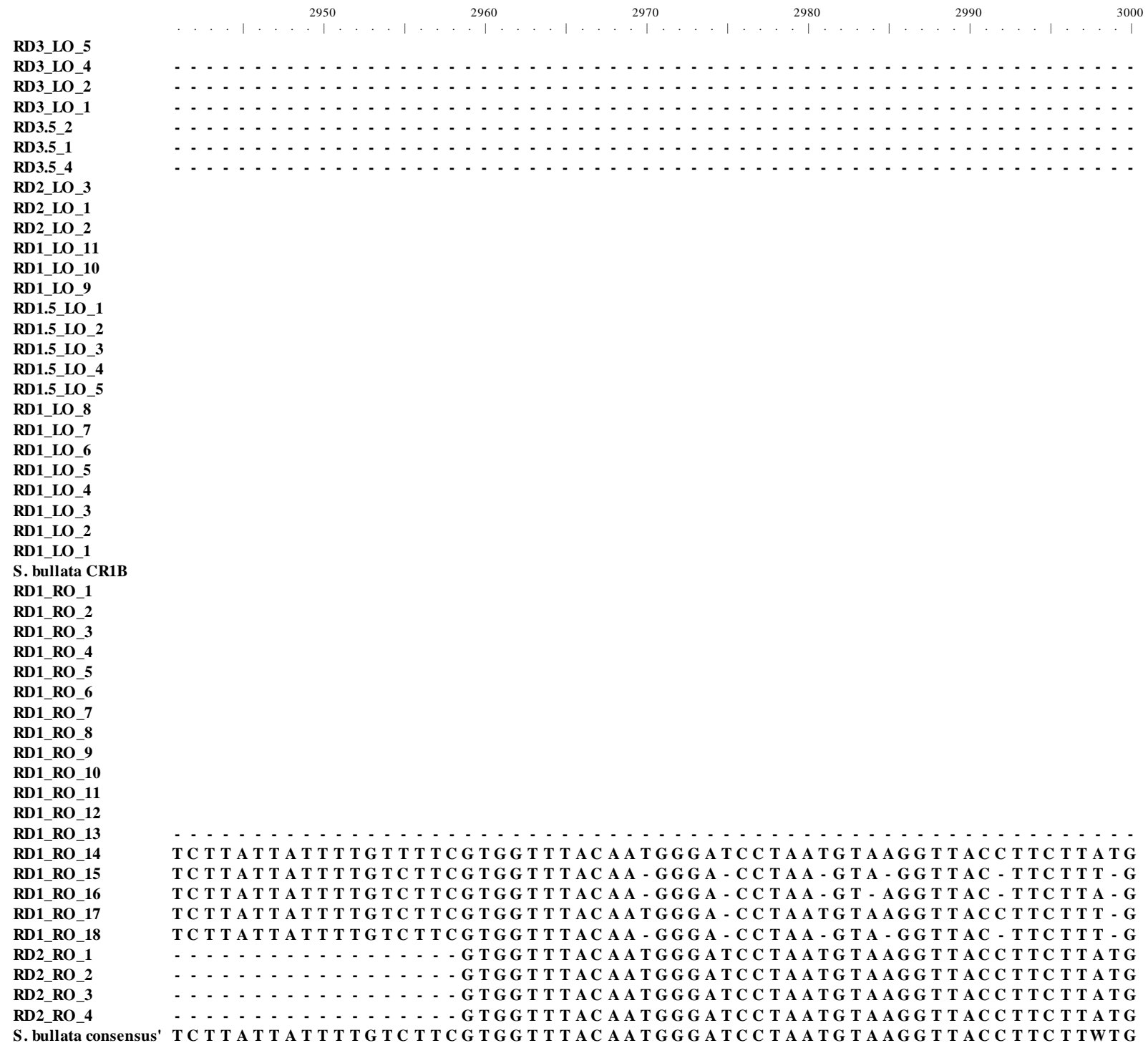




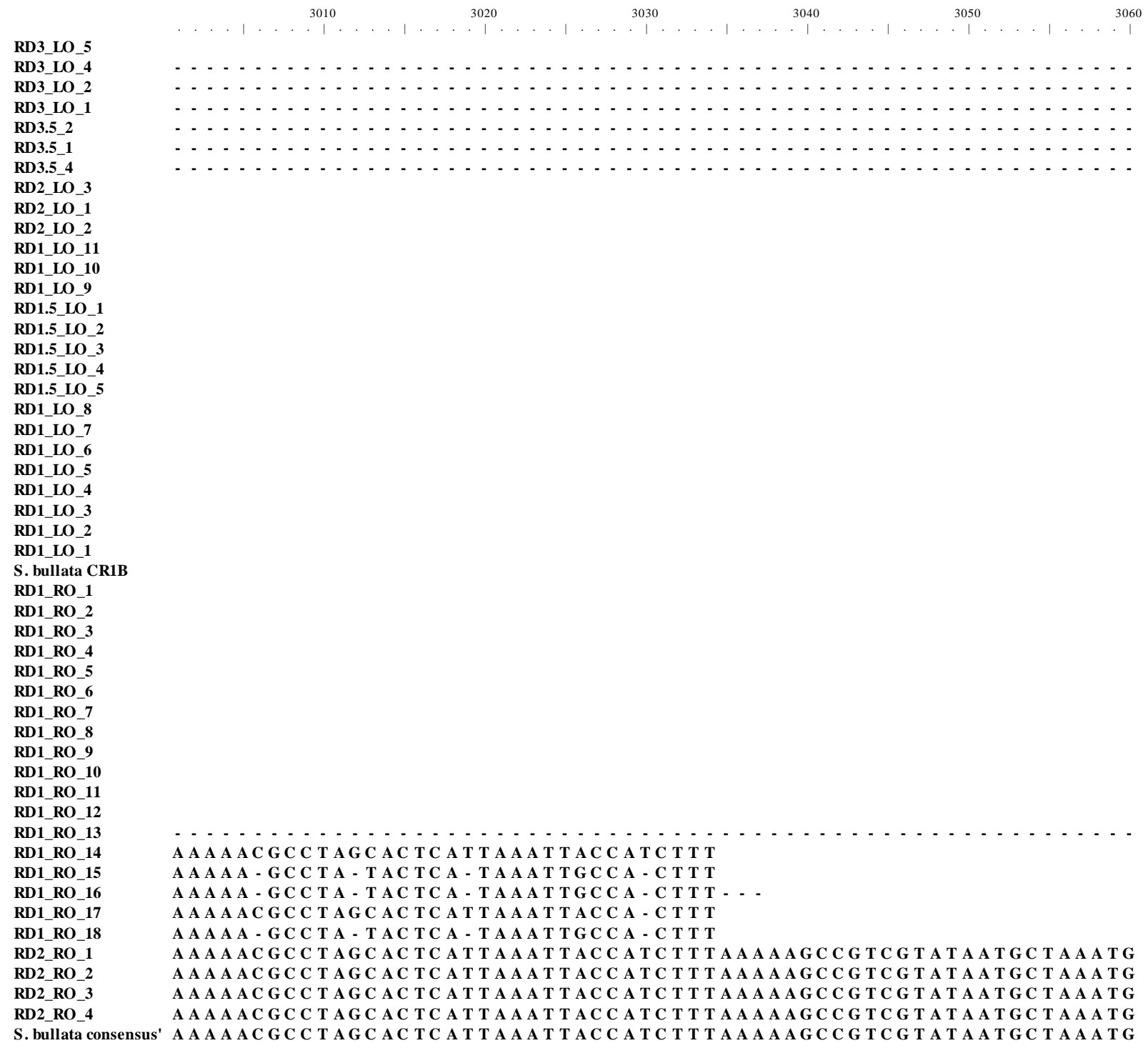




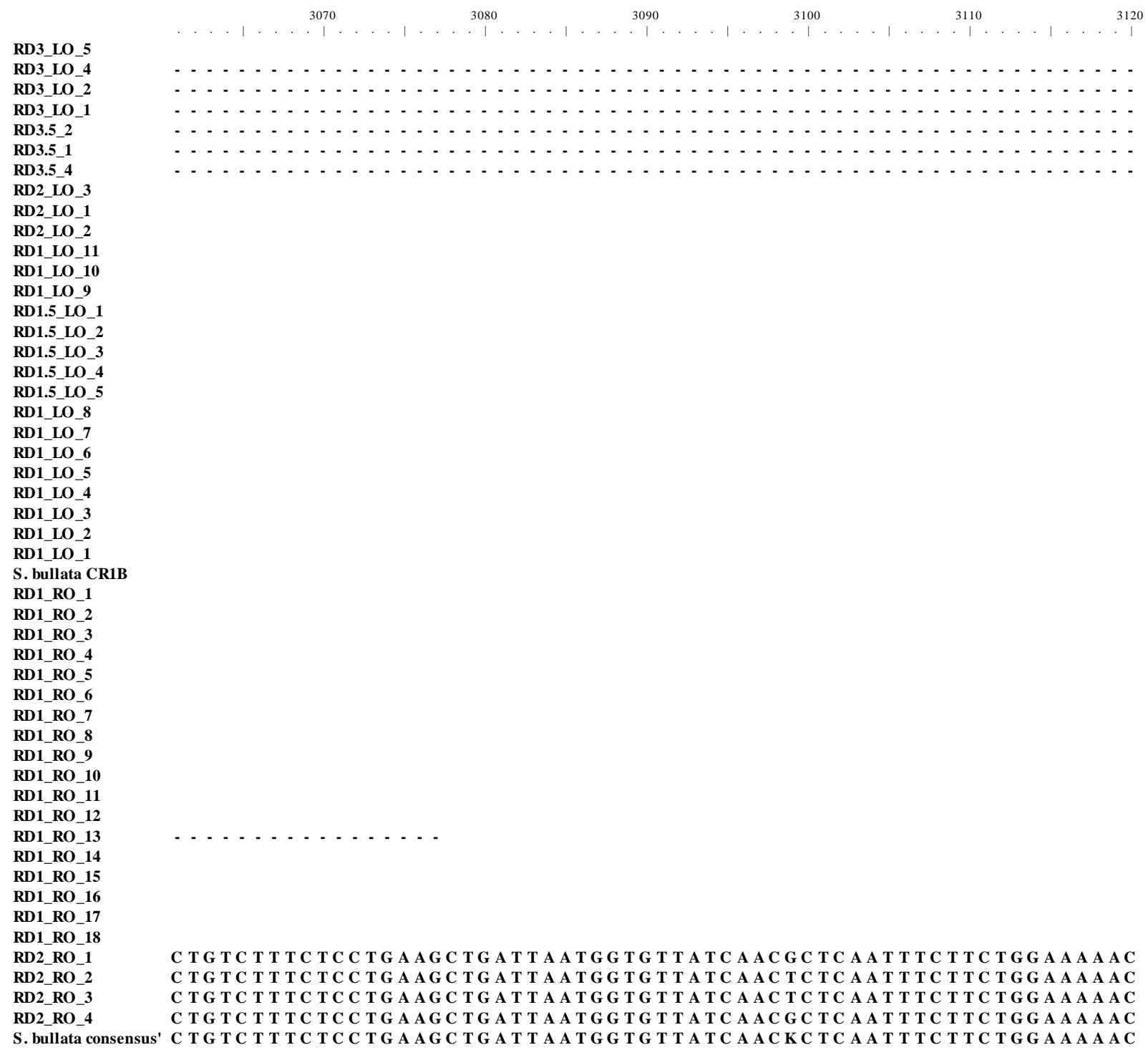




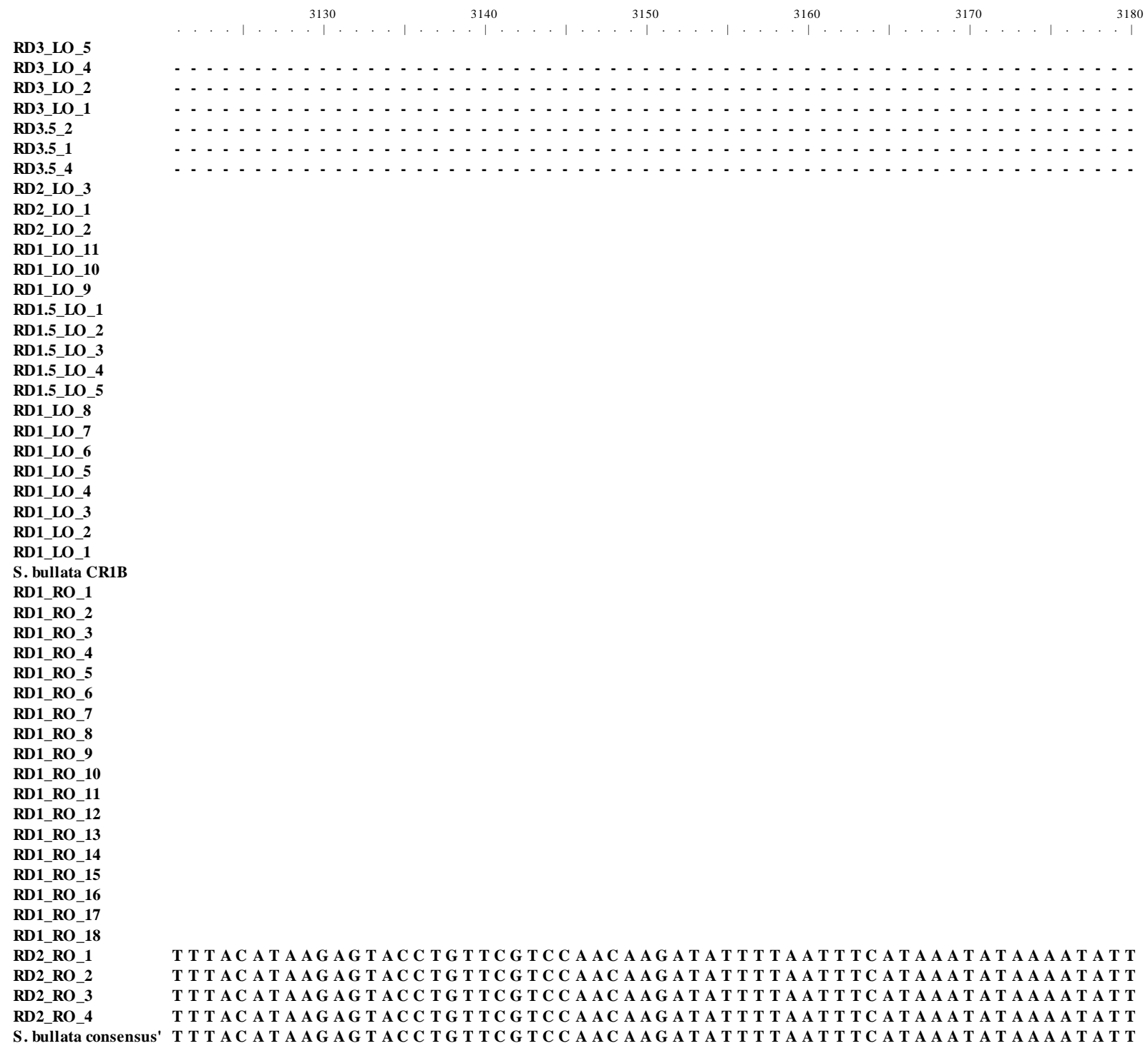




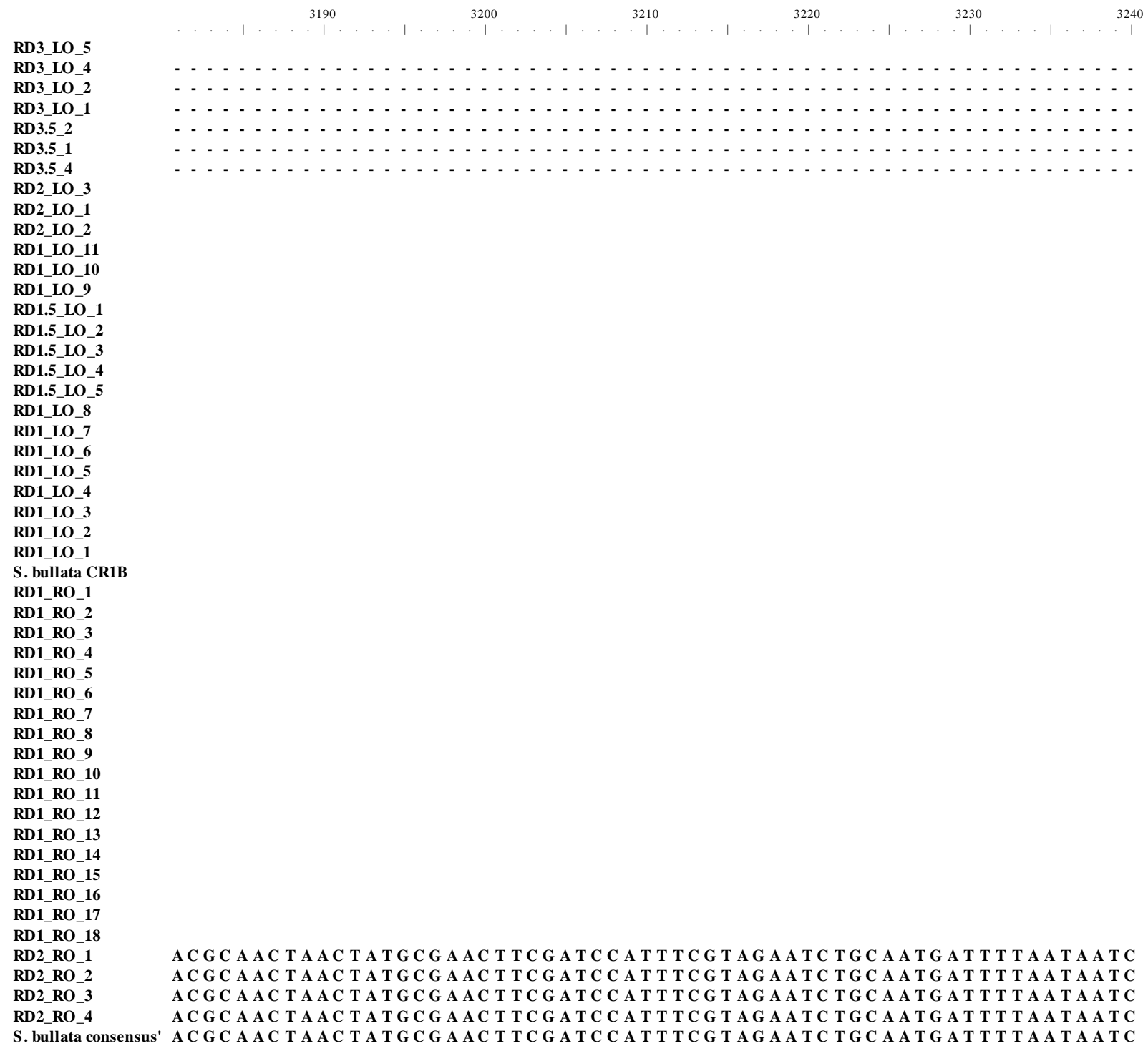




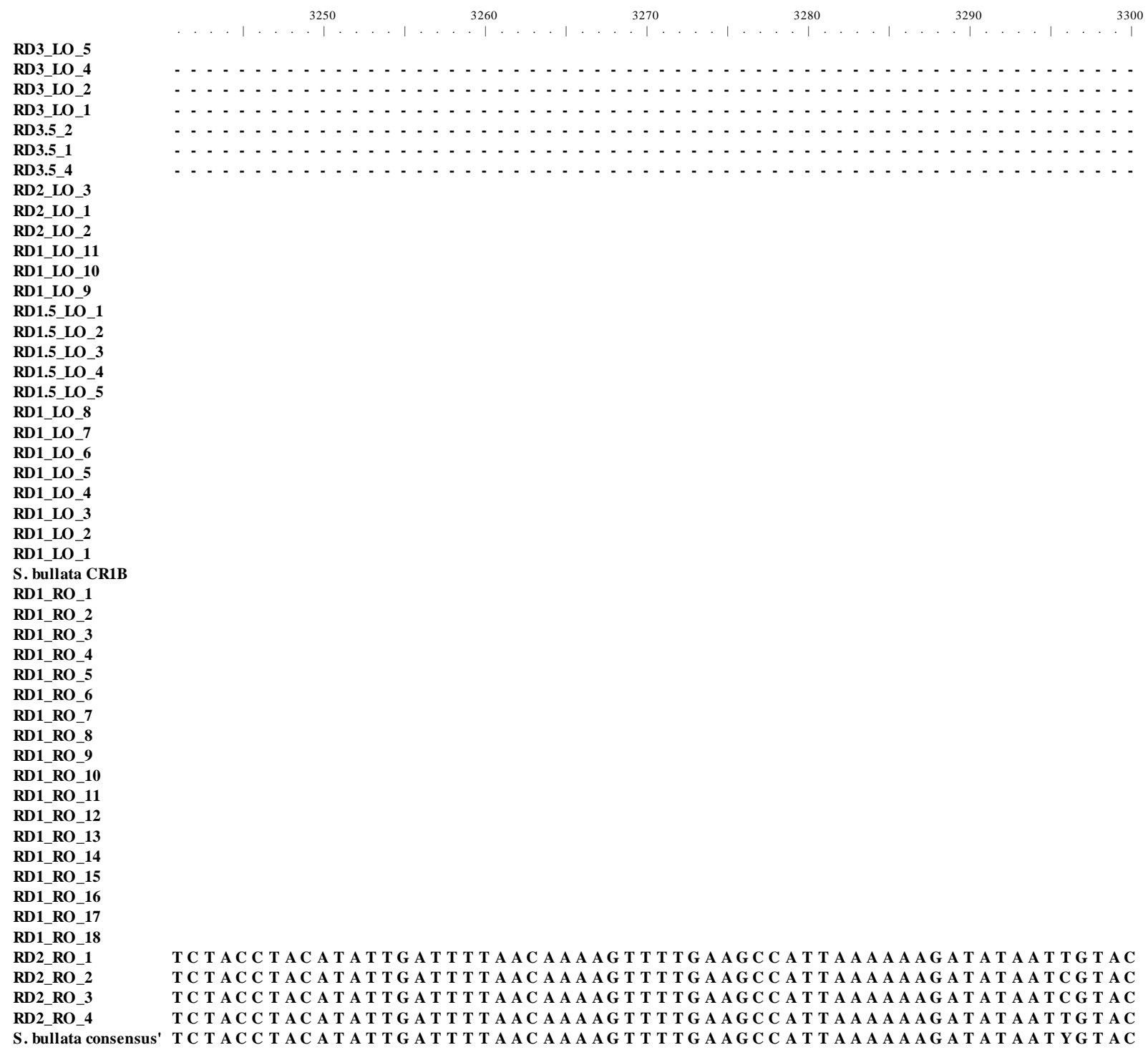




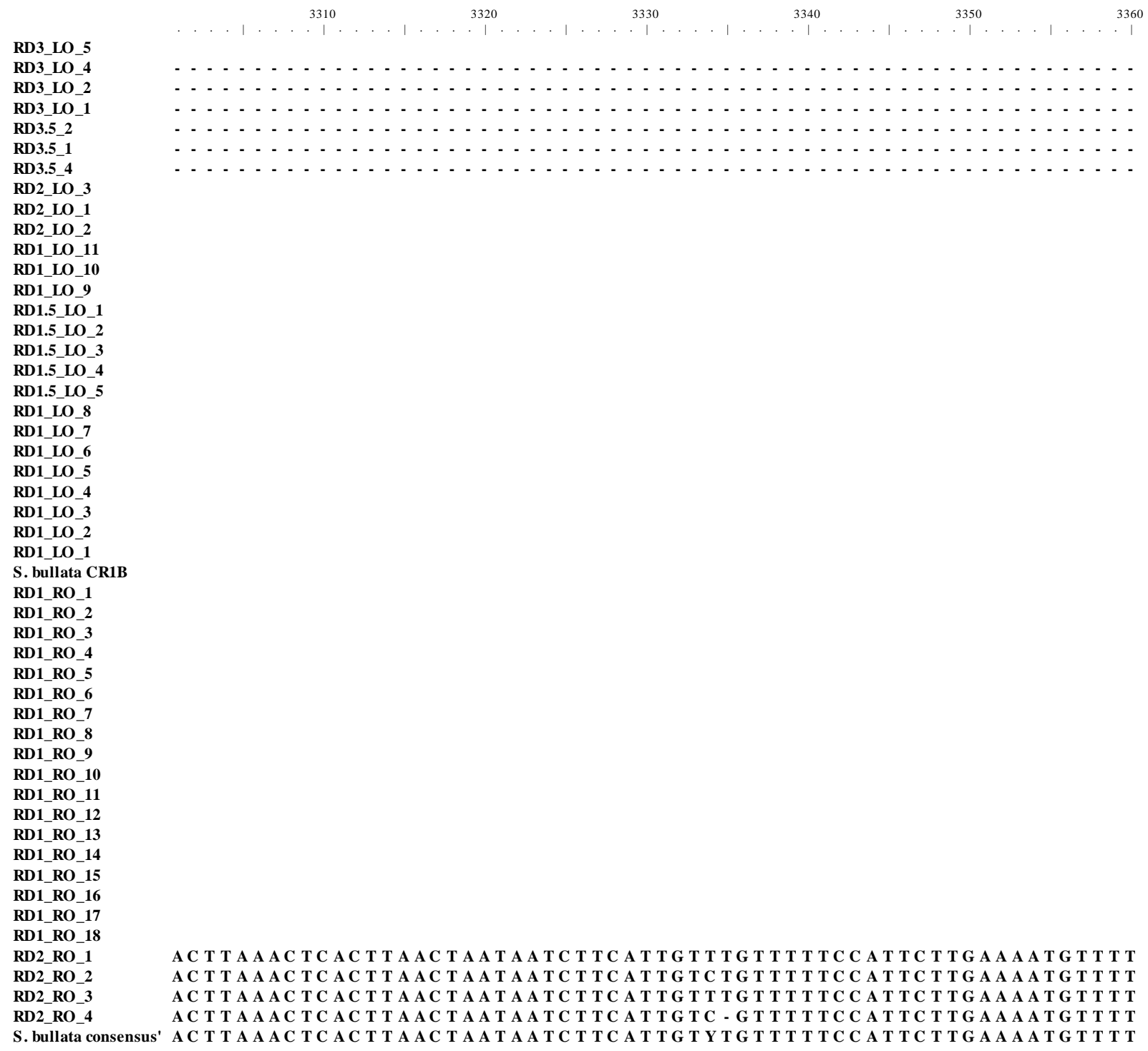




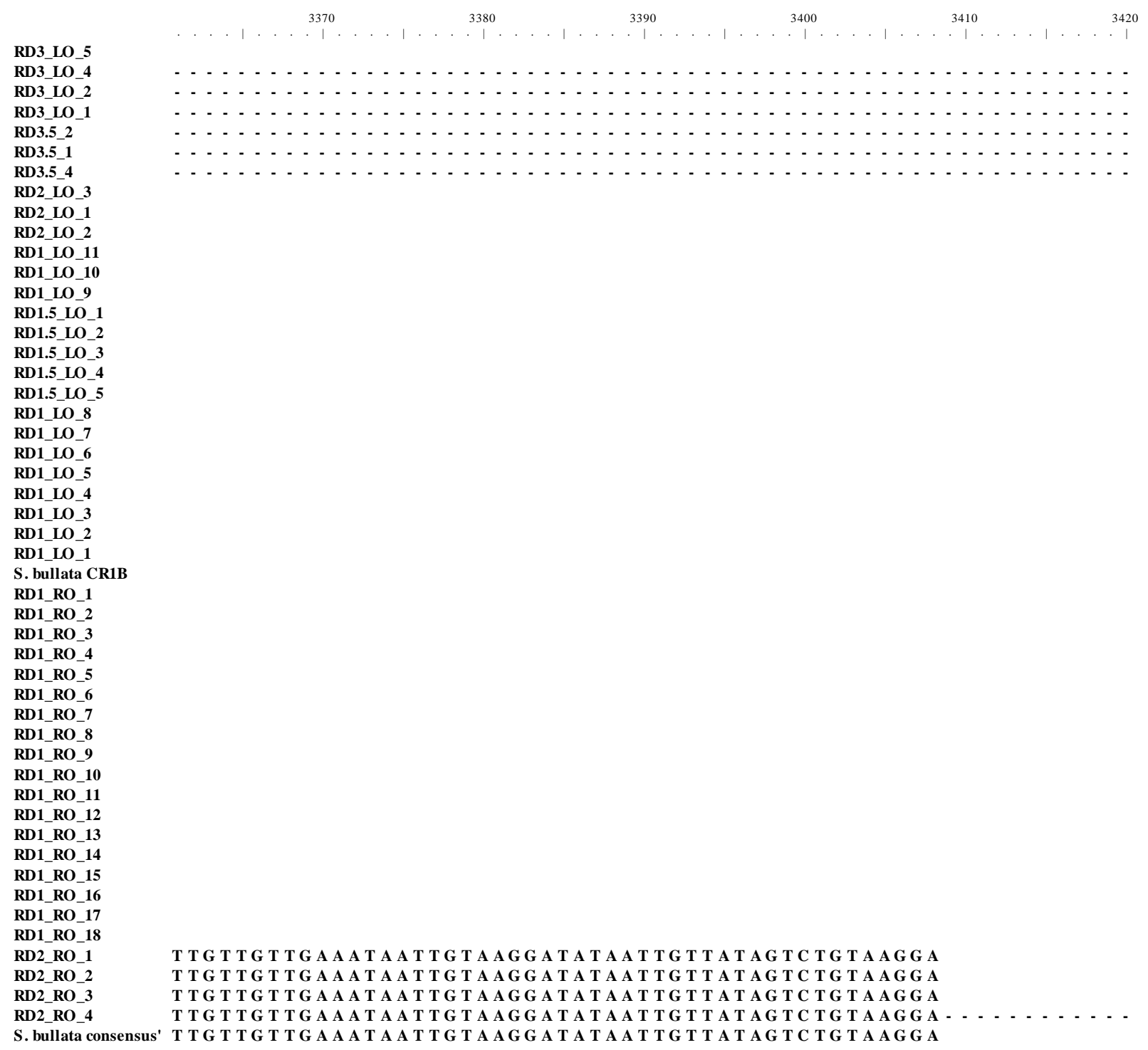

Supplemental Figure 6 Full sequence obtained of ORF2 from S. bullata. 